\title{
Energy Modelling and Fairness for Efficient Mobile Communication
}

\author{
by
}

\author{
Ekhiotz Jon Vergara
}

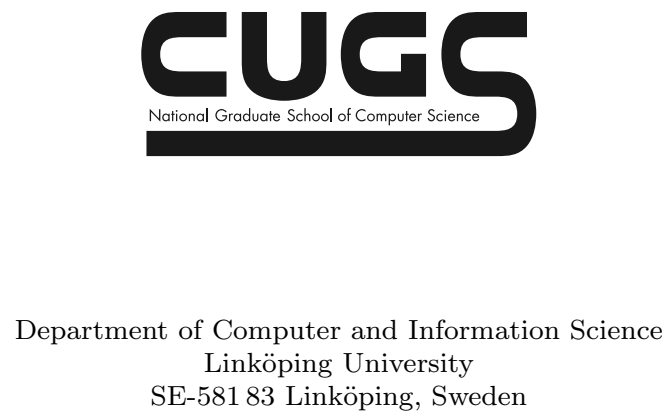

Linköping 2016 
Copyright (C) 2016 Ekhiotz Jon Vergara

ISBN 978-91-7685-822-6

ISSN $0345-7524$

Cover art by Ekhiotz Jon Vergara

Original photography by Josan Alonso, San Vicente de la Barquera https://www.flickr.com/photos/josanalonso/15891907372/

Printed by LiU Tryck 2016

URL: http://urn.kb.se/resolve?urn=urn:nbn:se:liu:diva-124538 


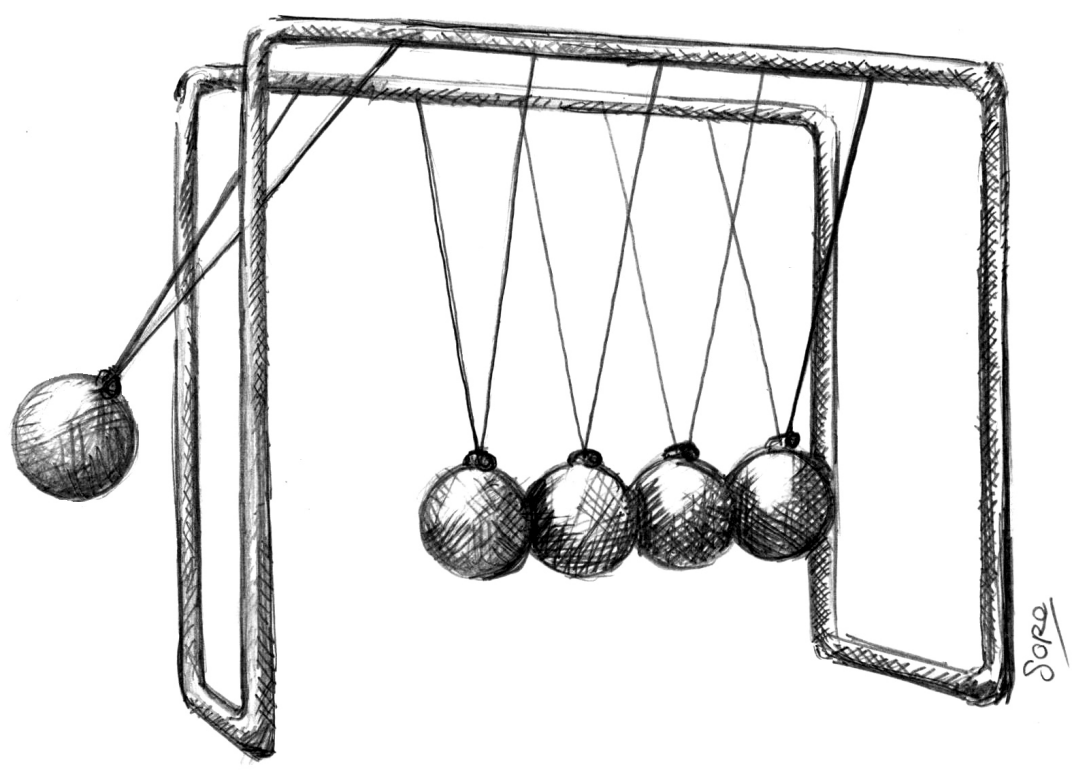





\section{Abstract}

Energy consumption and its management have been clearly identified as a challenge in computing and communication system design, where energy economy is obviously of paramount importance for battery powered devices. This thesis addresses the energy efficiency of mobile communication at the user end in the context of cellular networks.

We argue that energy efficiency starts by energy awareness and propose EnergyBox, a parametrised tool that enables accurate and repeatable energy quantification at the user end using real data traffic traces as input. EnergyBox offers an abstraction of the underlying states for operation of the wireless interfaces and allows to estimate the energy consumption for different operator settings and device characteristics. The tool is used throughout the thesis to quantify and reveal inefficient data communication patterns of widely used mobile applications.

We consider two different perspectives in the search of energy-efficient solutions. From the application perspective, we show that systematically quantifying the energy consumption of design choices (e.g., communication patterns, protocols, and data formats) contributes to a significantly smaller energy footprint. From the system perspective, we devise a cross-layer solution that schedules packet transmissions based on the knowledge of the network parameters that impact the energy consumption of the handset. These attempts show that application level decisions require a better understanding of possible energy apportionment policies at system level.

Finally, we study the generic problem of determining the contribution of an entity (e.g., application) to the total energy consumption of a given system (e.g., mobile device). We compare the state-of-the-art policies in terms of fairness leveraging cooperative game theory and analyse their required information and computational complexity. We show that providing incentives to reduce the total energy consumption of the system (as part of fairness) is tightly coupled to the policy selection. Our study provides guidelines to select an appropriate policy depending on the characteristics of the system.

This work has been supported by the Swedish National Graduate School of Computer Science (CUGS), Sweden. 



\section{Populärvetenskaplig sammanfattning}

Mobila tjänster som utnyttar kommunikation över mobila nätverk är till stor nytta för mobiltelefonanvändare. Mobilanvändarna fokuserar på interaktionen med applikationerna och förväntar sig att kunna kommunicera när det behövs utan att tänka på batteriets livslängd. Dessvärre resulterar ineffektiv användning av trådlösa gränssnitt (t.ex. WiFi eller mobilnät) i snabb batteriurladdning. Energiförbrukning och energihantering är en av de stora utmaningarna för informationsteknologin just nu.

Den här avhandlingen behandlar energieffektiviteten i mobilkommunikation med fokus på de mobila enheterna och användarens upplevelse. Den energiförbrukning som orsakas av kommunikation är inte proportionell mot mängden data som skickas vilket beror på hur data skickas över tid. Det är därför av stor vikt att energieffektivisering ingår i utvecklingsprocessen för programvaran.

Avhandlingen bidrar med EnergyBox, ett verktyg som gör det möjligt att enkelt kvantifiera energiförbrukningen för datakommunikation. Verktyget simulerar energiförbrukningen i trådlösa gränssnitt med kunskap om de aspekter hos både mobila enheter och nätverksoperatörer som påverkar nätverkskommunikationen. EnergyBox har redan använts i forskning och företagsprojekt (t.ex. Spotify).

EnergyBox används i avhandlingen för att kvantifiera och föreslå nya förbättringar för populära mobila applikationer från två olika perspektiv. För det första visar vi att applikationsutvecklare kan förbättra energieffektiviteten genom att kvantifiera och analysera implementationsval som påverkar hur data skickas. Vi exemplifierar processen med populära mobila spel, instant messaging och platsdelningsapplikationer. För det andra, föreslår vi en strategi som balanserar fördröjning av datasändningar mot energiförbrukning baserat på operatörernas nätverksinställningar.

Slutligen studerar vi det generella problemet att bestämma hur mycket olika delar bidrar till den totala energiförbrukningen i ett givet system (t.ex. applikationer i en mobilenhet). Problemet liknar hur en kostnad eller vinst kan delas rättvist vilket inte alltid har en entydigt bästa lösning. Vår jämförelse av de ledande metoderna för att bestämma delförbrukning ger riktlinjer för att välja en metod beroende på systemets beteende. Vi visar att incitament för att minska den totala energiförbrukningen i systemet är 
beroende av metodvalet. En rättvisande fördelning av förbrukning är också nödvändigt för att ge insyn i systemets energiförbrukning och underlätta bedömningen av olika beståndsdelars energieffektivitet och deras samspel i systemet. 


\section{Acknowledgements}

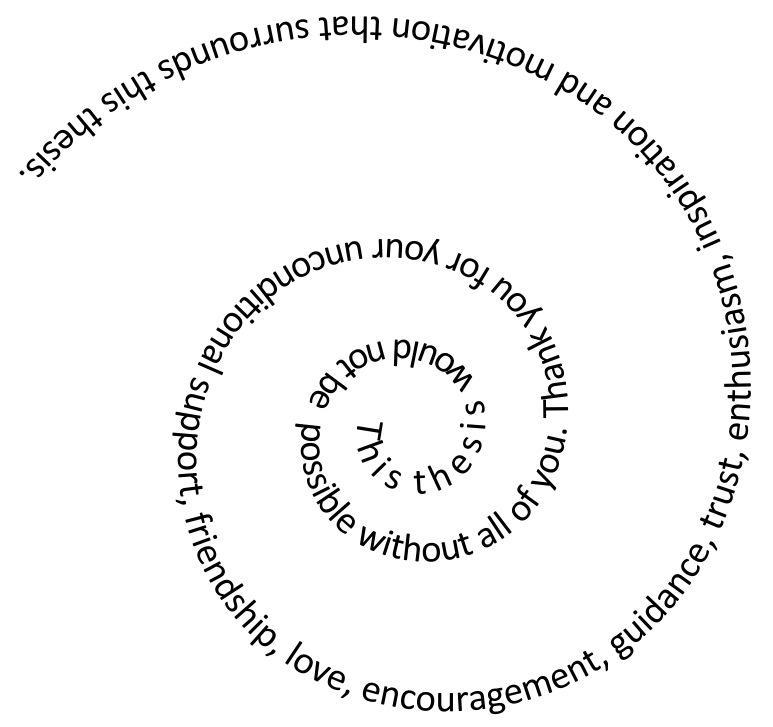


First of all, I am thankful to my main supervisor Simin Nadjm-Tehrani for giving me the opportunity to embark in this long journey, her energetic guidance, trust and the ability to push me further to improve my work. I extend my sincere gratitude to my secondary advisor Mikael Asplund for his enthusiasm and asking the opportune questions that make me see things from a different perspective.

I also thank my current and former RTSLAB fellows for their friendship, support, inspiring comments and stimulating discussions. Thanks also to my colleagues from other labs for enriching my days at university. A special mention goes to the lunch-box team and my personal Swedish teachers.

The administrative personnel, in particular Anne Moe, Eva Pelayo, and Åsa Kärrman, deserve my appreciation for efficiently helping me with the administrative tasks. I would also like to thank the students that decided to do their thesis under my supervision for their interest and effort. My gratitude also goes to the Swedish National Graduate School in Computer Science (CUGS) for every end of the month during all these years. I wish to thank the support of Ericsson AB, and in particular B-O Hertz, Pär Emanuelsson and Claes Alströmer for facilitating the measurement gathering phase.

Finally, for the encouragement, love, unconditional support, and making me forget about my work, I would like to tell my dear friends and family everywhere:

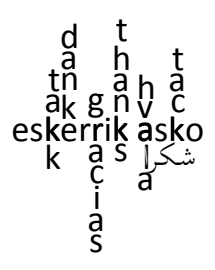

No puedo agradecer lo suficiente por todo el apoyo incondicional que he recibido de todos vosotros. Todo mi cariño y gratitud es para mi amatxo y aitatxo, y a su vez a mi familia en general. No podía faltar la mención a mis troopers, a los que les debo muchos de los buenos momentos de estos últimos años. Gracias a todos de corazón.

Nere bihotzeko hizkuntzan kuadrilari, lagunei eta familiari esker beroenak ematea ezin zan falta. Etxera noan bakoitzean zuekin elkartzea paregabea da. Azkenik, nere bizitzaren argiari bihotz-bihotzez eskerrak eman nahi dizkiot. Esker mila ez da nahikoa neregan ausardia behin eta berriro berpizteagatik, nere eguneroko zoriontasuna zaintzeagatik eta guztira hogeitasei kontutxo ederrengatik.

Nere esker onak guztioi emanez amaitzen det, besarkada handi bat eta elkar ikusi arte!

Ekhiotz Vergara Alonso

Linköping, Sweden

November, 2015 


\section{Contents}

1 Introduction $\quad 7$

1.1 Preliminaries ....................... 9

1.2 The search for energy efficiency . . . . . . . . . . . . . . 10

1.3 Mobile communication energy . . . . . . . . . . . . 11

1.4 Problem formulation . . . . . . . . . . . . . . . 13

1.5 Contribution .................... . . 14

1.6 Research methods . . . . . . . . . . . . . . 16

1.7 List of publications . . . . . . . . . . . . . . . . . . . . . . . . . . . . . 17

1.8 Thesis outline . . . . . . . . . . . . . . . . . . . . . 19

2 Background 2

2.1 Application communication energy . . . . . . . . . . . . 21

2.2 Technological background . . . . . . . . . . . . . 23

2.2.1 3G energy consumption . . . . . . . . . . . . 24

2.2.2 WiFi energy consumption . . . . . . . . . . . . 28

2.3 Overview of related works . . . . . . . . . . . . . 30

3 EnergyBox $\quad 33$

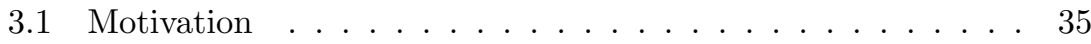

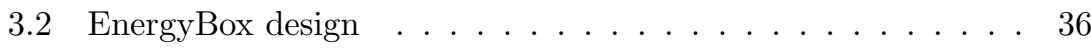

3.2 .1 3G model . . . . . . . . . . . . . . . 36

3.2 .2 WiFi model . . . . . . . . . . . . . . . 40

3.3 Evaluating EnergyBox accuracy . . . . . . . . . . . . 41

3.3.1 General methodology . . . . . . . . . . . . 41

3.3.2 Evaluation packet traces . . . . . . . . . . . . . 42

3.3.3 Selection of parameter values . . . . . . . . . . . . 43

3.4 Evaluation results . . . . . . . . . . . . . . . . . . . 45

3.4.1 Accuracy of EnergyBox 3G . . . . . . . . . . 46

3.4.2 Accuracy of EnergyBox WiFi . . . . . . . . . . . . . 48

3.5 Discussion and extensions . . . . . . . . . . . . . . . 49

3.5.1 Open source project . . . . . . . . . . . 50

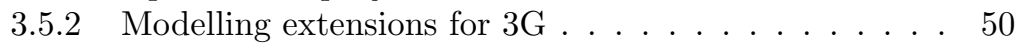

3.5.3 Long Term Evolution (4G) model . . . . . . . . . . 51

3.5.4 Cellular interface emulation over WiFi . . . . . . . . 51

3.5.5 Automated testing environment at Spotify . . . . . . . 51 
4 Communication Energy of Games 53

4.1 Mobile game dataset selection . . . . . . . . . . . . . . . . . 54

4.2 Evaluation setup and metrics . . . . . . . . . . . . . 55

4.3 Communication energy footprint of games . . . . . . . . . . 57

4.3.1 Energy consumption and data traffic . . . . . . . . . 57

4.3.2 UE states and data pattern . . . . . . . . . . . . . . 59

4.4 Beyond energy awareness . . . . . . . . . . . . . . . 61

5 Cross-Layer Burst-Buffering 63

5.1 Motivation ................... . . 64

5.2 Parameter inference algorithms . . . . . . . . . . . 65

5.2 .1 Inactivity timer estimation algorithms . . . . . . . . 66

5.2.2 RLC data buffer threshold estimation . . . . . . . . 68

5.2.3 Evaluation of inference algorithms . . . . . . . . . 70

5.3 Cross-layer burst buffering algorithm . . . . . . . . . . 72

5.4 Evaluation methodology . . . . . . . . . . . . . 75

5.4.1 Algorithm implementation . . . . . . . . . . . 76

5.4.2 Methodology to assess the total savings . . . . . . . 76

5.4.3 Algorithm benchmark methodology . . . . . . . . 78

5.5 Energy simulation results . . . . . . . . . . . . . 80

5.5.1 Network communication energy savings . . . . . . . 80

5.5.2 CPU energy footprint . . . . . . . . . . . . . . 82

5.5.3 Total energy savings . . . . . . . . . . . . . 83

5.6 CLBB vs. TailEnder . . . . . . . . . . . . . . . 83

5.7 Discussion . . . . . . . . . . . . . . 85

6 Energy-Efficient Location Sharing $\quad 89$

6.1 Scenario setup . . . . . . . . . . . . . . . 90

6.2 Evaluation settings . . . . . . . . . . . . . . . 93

6.3 MQTT vs. HTTP . . . . . . . . . . . . . . . . . . . . . . 93

6.3.1 Idle state . . . . . . . . . . . . . . . . . 94

6.3.2 Active state . . . . . . . . . . . . . . 95

6.4 Protocol for check-in . . . . . . . . . . . . . . . . . . . . . 98

6.5 Data encoding impact on energy . . . . . . . . . . . . . . 99

6.6 Summary and discussion . . . . . . . . . . . . . . 100

7 Energy-Efficient Instant Messaging 103

7.1 Collected IM dataset . . . . . . . . . . . . . . . . . . . 105

7.1 .1 Message origin . . . . . . . . . . . . 105

7.1.2 Message size . . . . . . . . . . . . . 107

7.1.3 Temporal properties ............... . 108

7.2 Bundling of messages . . . . . . . . . . . . . . . . . . . 110

7.3 Typing notification . . . . . . . . . . . . . . . . . . . 111

7.4 Evaluation methodology and settings . . . . . . . . . . . . 112

7.4.1 IM application . . . . . . . . . . . . . . . 113

7.4.2 Test traces and parameter settings . . . . . . . . 114 
7.5 Does message bundling pay off? . . . . . . . . . . . . . 116

7.5.1 Energy savings and bundling results . . . . . . . . . 116

7.5.2 Message delay . . . . . . . . . . . . . . . 118

7.6 Cost of typing notification . . . . . . . . . . . . . . . . 119

7.7 Summary and discussion . . . . . . . . . . . . . . . . 121

8 Energy Apportionment Policies $\quad 123$

8.1 Notation and background . . . . . . . . . . . . . . . 126

8.1.1 System model . . . . . . . . . . . . . . . 126

8.1.2 Energy apportionment policy . . . . . . . . . . 128

8.1.3 Game theory for energy apportionment . . . . . . . . 128

8.2 Energy apportionment policies . . . . . . . . . . . . 129

8.2.1 Energy-based policies . . . . . . . . . . . . . . 129

8.2 .2 Surrogate-based policies . . . . . . . . . . . . 133

8.3 Input and computational complexity . . . . . . . . . . 134

8.3.1 Required input information . . . . . . . . . . . . 134

8.3.2 Computational complexity . . . . . . . . . . . . 136

8.4 Fairness in energy apportionment . . . . . . . . . . . . 138

8.4.1 Fairness criteria . . . . . . . . . . . . . . 138

8.4.2 Fairness comparison . . . . . . . . . . . . . . . 139

8.4.3 Summary of the fairness analysis . . . . . . . . . . 143

8.5 Summary of the trade-offs and guidelines . . . . . . . . . . 143

8.5.1 General trade-offs . . . . . . . . . . . . . . . . 144

8.5.2 Properties of the system energy function . . . . . . . . 145

8.5.3 Selection guidelines . . . . . . . . . . . . . . 146

8.6 Summary and discussion . . . . . . . . . . . . . . 148

9 Related Works $\quad 151$

9.1 Energy measurement studies . . . . . . . . . . . . . 152

9.1.1 Cellular communication . . . . . . . . . . . . 152

9.1.2 WiFi communication . . . . . . . . . . . . 155

9.1.3 Bluetooth .................. 156

9.2 Energy models . . . . . . . . . . . . . . . . . . . 156

9.2.1 Measurement-based models . . . . . . . . . . 156

9.2.2 Analytical models . . . . . . . . . . . . . . 158

9.3 Energy accounting . . . . . . . . . . . . . . 159

9.3.1 Processing and operating systems . . . . . . . . . 159

9.3.2 Wireless sensor networks . . . . . . . . . . . . . 160

9.3.3 Energy-efficient buildings . . . . . . . . . . . . . 161

9.3.4 Mobile devices ................. . . 162

9.4 Energy management . . . . . . . . . . . . . . . . . 164

9.4.1 First energy-aware approaches . . . . . . . . . . . . 164

9.4.2 Current approaches in mobile devices . . . . . . . . 165

9.5 Energy saving techniques . . . . . . . . . . . . 165

9.5.1 Traffic adaptation techniques ........... . 165

9.5.2 Link selection techniques . . . . . . . . . . . 169 
9.5.3 Interface power saving mechanisms . . . . . . . . . 170

9.5.4 Network level techniques . . . . . . . . . . . . . . 170

10 Conclusion and Future Work 175

10.1 Conclusions . . . . . . . . . . . . . . . . . 175

10.2 Future work . . . . . . . . . . . . . . . . 179

$\begin{array}{lr}\text { Appendices } & 183\end{array}$

$\begin{array}{ll}\text { A Recharging smartphones } & 185\end{array}$

$\begin{array}{ll}\text { B Measurement setup } & 187\end{array}$

$\begin{array}{ll}\text { C IM Application Baseline } & 191\end{array}$

$\begin{array}{lr}\text { D Fairness Property Proofs } & 193\end{array}$

D.1 Symmetry . . . . . . . . . . . . . . . . . . . 194

D.2 Dummy player . . . . . . . . . . . . . . . 196

D.3 Individual rationality . . . . . . . . . . . . . . . . . 202

D.4 Coalitional rationality . . . . . . . . . . . . 205

D.5 Entity desirability . . . . . . . . . . . . . . 206

D.6 Activity-based fairness . . . . . . . . . . . . . . . . 209

D.6.1 Activity-based symmetry . . . . . . . . . . . . . 210

D.6.2 Activity-based dummy player . . . . . . . . . . . . 212

D.6.3 Activity-based individual rationality . . . . . . . . . . 212

D.6.4 Activity-based entity desirability . . . . . . . . . . 212 


\section{List of Figures}

1.1 Common energy resource and individual energy resource system interpretations. . . . . . . . . . . . . . . . 10

1.2 Simplified system illustration. . . . . . . . . . . . . . . . 12

1.3 Simplified system illustration and the mapping to the contributions, chapters and the focus of each chapter. . . . . . . . . . . . 20

2.1 Communication energy and standard deviation of different Instant Messaging applications for the short conversation. . . . . . . . 22

2.2 Data pattern characteristics of the different Instant Messaging applications for the short conversation. . . . . . . . . . . . . 23

2.3 RRC state machine as depicted in Ericsson's labs [72]. . . . . . . . 24

2.4 Example $3 \mathrm{G}$ power profile for the operator TeliaSonera. The RRC states are annotated. . . . . . . . . . . . . . 26

2.5 Example $3 \mathrm{G}$ power profile for operator 3. . . . . . . . . . . . 27

2.6 Example power profile and data communication for a Skype chat session and the TeliaSonera operator. . . . . . . . . . . . . . 28

2.7 Power levels of adaptive PSM for Samsung Galaxy SII and Sony Ericsson Xperia Arc. . . . . . . . . . . . . . . . . . . . . . . . 29

2.8 Power levels of adaptive PSM for the Sony Ericsson Xperia Arc. $\quad 30$

2.9 Taxonomy of wireless communication energy at the user end. . . . 30

3.1 Overview of EnergyBox function. . . . . . . . . . . . . . . 34

3.2 Overview of the state machines for 3G (a) and WiFi (b). . . . . 37

3.3 Measured time to empty the buffer $T_{e}^{u}$. . . . . . . . . . . . 44

3.4 Energy consumption of the measured trace and EnergyBox 3G estimation for different traces. . . . . . . . . . . . . . . . . 46

3.5 A fragment of a $3 \mathrm{G}$ measurement and EnergyBox 3G inferred output for the Email trace. . . . . . . . . . . . . . . . . . 46

3.6 A fragment of a $3 \mathrm{G}$ measurement and EnergyBox 3G inferred output for the Spotify trace. . . . . . . . . . . . . . . . 47

3.7 3G time accuracy for all the traces (left) and time spent by the UE in each state for Skype Chat and Web 2 (right). . . . . . . . . 47

3.8 Energy consumption of the measured trace and EnergyBox WiFi estimation for different traces. . . . . . . . . . . . . . . . . . . 48

3.9 WiFi time accuracy for all the traces (left) and time spent by the station in each state for Web and Spotify (right). . . . . . . . .

4.1 Average energy consumption and standard deviation for the selected games resulted from playing each game 5 times for 15 minutes. 57 
4.2 Average data exchanged and standard deviation for the 20 games.

4.3 Scatter plot showing the average energy consumption and data sent for the 20 games. . . . . . . . . . . . . . . . .

4.4 Distribution of time spent by the UE in the different RRC states for each game. . . . . . . . . . . . . . . . 60

4.5 Number of RRC state transitions for the 20 games. . . . . . . . . . 61

5.1 The effect of small packet aggregation on energy consumption. . . 65

5.2 RTT values obtained for $T_{1}$ inference. In the example InterPacketInterval is set to 0.5 seconds and TestPeriod of $T_{1}$ is 7 seconds. . .

5.3 Illustration of lines 18-34 of Algorithm 2 showing the packets sent, their size and the state of the UE. . . . . . . . . . . . . 70

5.4 Comparison of algorithms in terms of energy consumption and elapsed time. . . . . . . . . . . . . . . . 71

5.5 Characteristics of the emulated traces. . . . . . . . . . . . 77

5.6 Measurement-based CPU model. . . . . . . . . . . . . . . 78

5.7 Overview of the TestFramework running in the test computer of the evaluation environment. . . . . . . . . . . . . . . 79

5.8 Network communication energy savings. . . . . . . . . . . . . . 81

5.9 Percentage of time spent by the UE in the different $3 \mathrm{G}$ states over the total time of the original traces (y axis) scheduled with different $T_{w}$ (seconds) by the KLS (x-axis). . . . . . . . . . . . .

5.10 Example of CPU energy consumption by running the KLS (left) and CPU energy footprint for the different traces (right). . . . .

5.11 Network transmissions energy savings, KLS energy overhead and total energy savings as a percentage of no-KLS base energy consumption. . . . . . . . . . . . . .

5.12 Energy savings of CLBB and TailEnder for operator 1. . . . . . . 84

5.13 Energy savings of CLBB and TailEnder for operator 2. . . . . . . 85

5.14 Energy consumption examples of background traffic and energyefficient shaped background traffic. . . . . . . . . . . . . . 86

6.1 Overview of the data flow in a location sharing service based on cellular (3G) communication. . . . . . . . . . . . . . 90

6.2 Location Sharing Application interaction. . . . . . . . . . . . . . . 92

6.3 Amount of data traffic and energy consumption for HTTP and MQTT techniques in the idle state. . . . . . . . . . . .

6.4 Amount of data traffic for the HTTP and MQTT techniques in active state. . . . . . . . . . . . . . . . .

6.5 Empirical CDF of packet size and inter-packet interval of HTTP and MQTT (9 users and $T=15 \mathrm{~s}$ scenario). . . . . . . . . 96

6.6 Energy consumption for the HTTP and MQTT techniques in active state. . . . . . . . . . . . . . . . . .

6.7 Percentage of time spent by the UE in the different RRC states over the experiments for HTTP and MQTT. . . . . . . . . . . .

6.8 Empirical CDF of inter-update interval in hours and minutes of the $22,387,930$ check-ins from 224,804 users. . . . . . . . . . . .

6.9 Energy consumption (left) and user equipment RRC states for different data encodings (right). . . . . . . . . . . . . . . . . 100 
7.1 Message classification per user. . . . . . . . . . . . . . . 106

7.2 Message distribution of selected users over their chats (left) and overall message size distribution (right). . . . . . . . . . . . . 107

7.3 Distribution of the total data sent over the message size. . . . . . . 108

7.4 Normalised number of messages over hours in a day (top-left), empirical CDF of inter-message interval (top-right) and normalised number of messages during different days of a week (bottom). . . . 109

7.5 Average and standard deviation of messages per day per user. . . . 110

7.6 Architecture of the IM prototype implementation and the test environment. . . . . . . . . . . . . . . . . . . . . . . 113

7.7 Test conversations. . . . . . . . . . . . . . . . . . . . . . 115

7.8 Normalised average energy and standard deviation for the effect of message bundling for the different conversations. . . . . . . . . 116

7.9 Box plot of the delay experienced by the different messages for Sparse and Dense conversations. . . . . . . . . . . . . . . 119

7.10 Normalised average energy and standard deviation of the typing notification feature for the different conversations. . . . . . . 120

8.1 Energy apportionment example for a simplification of a common system. . . . . . . . . . . . . . . . . . . . . 124

8.2 Example of the time model for two discrete signals and a tuple. . . 128

9.1 Taxonomy of wireless communication energy at the user end. . . . 152

9.2 Traffic aggregation and batching. . . . . . . . . . . . 167

A.1 Energy production of the world's nuclear power plants. . . . . . . 186

B.1 Measurement setup for 3G and WiFi. . . . . . . . . . . . 187

B.2 Aggregated power consumption for CPU and network transmission. 189

B.3 Stabilised CPU power trace and a network transmission. . . . . . . 189

C.1 Energy consumption of the basic implementation compared to popular IM applications when sending the Short conversation. . . . . . 191

C.2 Time spent in the different RRC states of the basic implementation compared to popular IM applications when sending the Short conversation. . . . . . . . . . . . . . . . .

D.1 Counterexample used in the proofs for symmetry for Policies 9 and $10 \ldots \ldots \ldots \ldots \ldots \ldots \ldots \ldots$

D.2 Illustration of the counterexample used in the proofs for dummy player for Policies 9 and 10. . . . . . . . . . . . .

D.3 Illustration of the counterexample used in the proof for null player for Policy 10. . . . . . . . . . . . . . . . . . .

D.4 Illustration of the counterexample used in the proofs for individual rationality for Policies 8, 9 and 10. . . . . . . . . . . . 204

D.5 Counterexample used to show that Policies 8, 9 and 10 do not satisfy entity desirability. . . . . . . . . . . . . . . . . 208

D.6 Counterexample used in the proofs for activity-based symmetry for Policy 10. . . . . . . . . . . . . . . . . . . . . . 211 
D.7 Counterexample showing that Policy 10 does not satisfy entity desirability.

\section{List of Tables}

1.1 Mapping between the contributions and the general solutions. . . .

2.1 Short conversation. . . . . . . . . . . . . . . . .

2.2 State transitions and triggering packet sizes for TeliaSonera measured in July 2012. . . . . . . . . . . . . . . . . . . . . . . .

3.1 Input parameters for EnergyBox $3 \mathrm{G}$ and the values used for the evaluation. These parameters correspond to the Ericsson F337 broadband module connected to the TeliaSonera network at our location from March 2012 to April 2013. . . . . . . . . . . . . . . .

3.2 Input parameters for EnergyBox $\mathrm{WiFi}$ and the values used for the evaluation. These parameters correspond to a Samsung Galaxy SII, which does not implement the PSM-TX state. . . . . . . . . . 40

4.1 Single player games with time-insensitive input. . . . . . . . . . . 56

4.2 Single player games with time-sensitive input. . . . . . . . . . . . 56

4.3 Multiplayer online games. . . . . . . . . . . . . . . 56

5.1 CLBB parameters for two different operators. . . . . . . . . . . . 80

7.1 Message origin per direction and chat type. . . . . . . . . . . 106

7.2 Number of bundles and bundles per message for the Dense conversation. . . . . . . . . . . . . . . . . . 117

7.3 Number of bundles and bundles per message for the Sparse conversation. . . . . . . . . . . . . . . . . 118

8.1 Summary of the 10 energy apportionment policies and their intuition.130

8.2 Overview of the considered energy apportionment policies. . . . . 135

9.1 Summary of the works considering network level techniques for mobile devices. . . . . . . . . . . . . . . . . . . . . 172

D.1 Reference to the fairness properties results for each policy. . . . . . 193

D.2 Two entity game with symmetric entities. . . . . . . . . . . . 195

D.3 A game where entity 2 is a dummy player. . . . . . . . . . 196

D.4 Activity function values for the counterexample. . . . . . . . . 197

D.5 A counterexample used to show that Policy 9 does not satisfy then null player property. . . . . . . . . . . . . . . . . 201

D.6 An example where Policy 3 does not satisfy individual rationality. 202 
D.7 A counterexample used to show that Policies 8, 9 and 10 do not always satisfy individual rationality. . . . . . . . . . . . . 203

D.8 Counterexample to prove that Policy 4 does not satisfy coalitional rationality. . . . . . . . . . . . . . . . . . 205

D.9 A counterexample used to show that Policies 8, 9 and 10 do not satisfy entity desirability. . . . . . . . . . . . . . 208 
"Even if you don't have the perfect idea to begin with, you can likely adapt."

Victoria Ransom, Wildfire

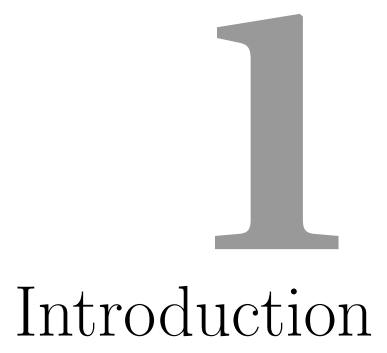

With the advent of computationally powerful handsets and mobile services we are in the dawn of the "information anytime anywhere" era. Wireless communication is a major form of communication, and true mobility will increasingly be supported by widespread deployments of cellular infrastructures. Unfortunately, mobile users still experience short battery lifetimes, making the energy consumption the Achilles' heel of user quality of experience. Energy consumption and its management is a challenge in computing and communication system design, and it is of paramount importance for battery driven devices. Battery lifetime has become an essential aspect in mobile communication.

While mobile traffic is forecasted to continue experiencing tremendous growth [71], this growing wave of mobile data communication has several extreme consequences: (1) unforeseen data volumes make the mobile operators eager to squeeze every bit of the possible capacity and minimise the cost of their infrastructure, (2) the significant increase in the use of the radio hardware interfaces in mobile devices results in shorter battery lifetimes, and (3) the mass diversity of applications and platforms create an ecosystem that is hard to optimise from every conceivable perspective. The main characters playing a role in this complex ecosystem are the mobile device vendors, application developers, cellular network operators and the end users.

While the technology development and the device manufacturers may provide more energy-efficient hardware and batteries in the longer run, the lack of energy awareness on the software front has become all too apparent. Even though manufacturers are likely to optimise the built-in software, their devices are shipped to a market where they have no control over the wide 
range of applications that can be used on them. Most current applications are completely oblivious to how their operation and data transmissions affect the energy consumption of the device. Mobile device manufacturers have argued that their hardware systems are energy-efficient and that the energy inefficiencies are in the higher level software [234,237]. Thus we believe that there is still a need for carefully analysing the energy footprint of application software, and to use software to monitor and reduce the energy consumption.

At the other end, the efforts of cellular operators are directed towards lowering energy consumption in the infrastructure nodes, not especially caring about the user end. This might seem natural since a great part of the operational cost of the network is due to energy consumption ( 1 billion euro per year [34]), and a typical base station consumes several magnitudes more power than a mobile handset [21] (more than $1 \mathrm{~kW}$ for a macro base station, around $800 \mathrm{~W}$ for a micro base station, and $14 \mathrm{~W}$ for a pico base station, whereas a current smartphone's maximum consumption does not reach $6 \mathrm{~W})$.

A user that has access to the data and services anytime anywhere will focus on interactions with the applications, expecting to communicate whenever needed without thinking about battery lifetime. However, the above ecosystem leaves the user in an undesirable state. Cellular networks impose high energy consumption on the mobile device due to the radio resource allocation performed at the operator end. We find that the battery of the users is wasted by the undisciplined use of data transmissions in the cellular network, using applications that were not specifically optimised for a given radio interface or operator settings. Battery lifetime has become the least satisfying factor in mobile devices and a decisive factor when selecting the device model or manufacturer [187]. Mobile users already show their dissatisfaction through negative reviews referring to energy consumption in application stores [259]. Seen from a user perspective, what counts in the end is that the handset has a long enough battery lifetime.

Last but not least, the aggregated consumption of recharging all the smartphones becomes a significant cost. Given the large number of mobile subscribers (more than 3 billion unique subscribers and more than 7 billion total subscribers [89]) and smartphone shipments (1.3 billion by the end of 2014 [109]), one can estimate that the average cost of recharging these devices per year is equivalent to the power generated by a small nuclear power plant or the annual consumption of 354,609 households (see Appendix A for details about the estimations).

With the above situation in mind, energy economy in mobile devices is an important factor, and wireless interfaces account for a great energy cost on mobile devices $[84,173]$. There is a need to reduce the energy consumption of wireless data communication at the user end and energy awareness is the key starting point to achieve energy efficiency. Energy agnostic systems and applications are likely to waste energy in inefficient transmissions by not being informed of how their transmissions impact the battery lifetime. 
Similarly to other contexts, energy-aware design and operation are required in order to achieve energy-efficient mobile communication systems.

In the next section we describe concepts and challenges in energy efficiency in computer and communication systems, introduce our system categorisation and explain general reasons and solutions for energy waste. Then, in section 1.3, we restrict the scope to communication energy in mobile devices to state the problem formulation and contributions of the thesis.

\subsection{Preliminaries}

Energy consumption is characterised by its global system nature, which can be divided into two main dimensions [272]: components and entities. A component is a unit of the system which consumes energy (e.g., hardware such a CPU or a network interface). A system is composed of different components that can consume energy concurrently and may employ different power management techniques. For example, a mobile device can be considered a system composed by several components such as CPUs, wireless interfaces or a screen.

An entity is an abstraction to which resources provided by the components are allocated (e.g., CPU time or network bandwidth) and against which the resulting energy usage is charged [113]. An entity performs activities which may span different components causing the system that they are part of to consume energy. Examples of entities in a mobile device are applications, processes or logical sets of operations whose resource usage should be grouped together [83]. The energy consumption of a system is determined by component usage patterns and interactions between the coexisting entities and components.

In our work we categorise two different types of system interpretations that abstract the system components and focus on the entity end. An energy resource supplies energy to the system, such as a battery for a mobile device. Figure 1.1 shows the two system interpretations: (1) common energy resource system, where the entities share a common energy resource, and (2) individual energy resource system, where each entity has an energy resource. The two interpretations are abstractions that facilitate the analysis of a scenario by setting the system boundaries. For example, a mobile device is a common resource system if applications are the entities of interest, whereas a mobile device is an entity with individual energy resource if the considered system is a network of mobile devices.

It is interesting to note that the total energy consumption of an individual energy resource system is determined by the sum of each entity's consumption. However, the contribution of each entity to the total consumption it is not necessarily its own consumption. An entity can contribute to other entities' consumption by being part of the system. For example, an entity's consumption is influenced by other entities in a network when overhearing transmissions. 
Common energy resource system

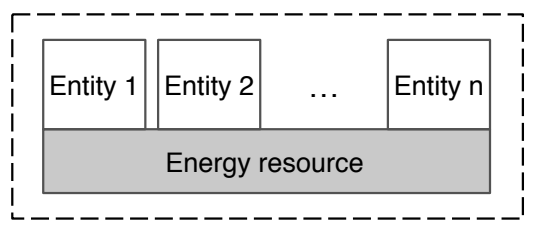

Individual energy resource system

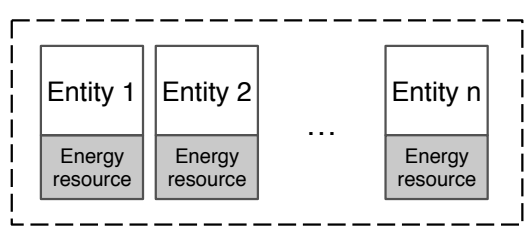

Figure 1.1: Common energy resource and individual energy resource system interpretations.

The energy consumption created by a resource usage at component level is already complex since (1) the amount of activity of an entity is often not proportional to the consumed energy, and (2) energy consumption does not necessarily correspond to the time interval in which the activity happened. Additionally, the coexisting activities in shared components created by the different entities in the system complicate the problem. This often results in the actual consumption not being the sum of what the entities would consume using the component alone.

\subsection{The search for energy efficiency}

In order to achieve energy-efficient systems, there is a need to analyse and optimise the energy efficiency of system's components as well as entities and their interaction. Energy proportionality is argued to be a desired feature of every energy-efficient system [27]. This is defined as the relation between system load or utilisation and the energy consumption. Energy proportionality enforces the energy consumption to be low when the utilisation is low, and the energy consumption proportionally increases with the utilisation. Unfortunately, we find that many systems are not energy proportional and consume high amounts of energy when the utilisation is low.

Throughout the thesis we argue that energy awareness is the key starting point to identify potential energy bottlenecks and efficiently diminish energy waste. Several general reasons explaining energy waste in computer systems have been identified [206], such as complex design trade-offs that lead to resource over-provisioning or modularity leading to local optimisations. General solutions have also been pinpointed in order to improve the energy efficiency of a system [206]. We relate our approaches and solutions in section 1.5 to some of the existing general practices, which we categorise into design stage and runtime solutions:

- Design stage solutions: The energy footprint can be reduced by using a more efficient alternative for the same task (e.g., select a more efficient hardware component), adopting holistic solutions to analyse 
the broad scope of the problem considering cross-layer interaction, optimising the resource usage for the common utilisation of the system, and considering only the minimum desired functionality and requirements.

- Runtime solutions: The following strategies can improve the energy efficiency of a system at runtime: Trade-off some other aspect for energy, disable or scale down unused resources, match utilisation to the resource characteristics, group multiple activities, spend someone else's power (e.g., offloading computation), and spend power to save power.

While the above practices are general, designing methods to apply them in real contexts and systems is far from trivial. We study some of these generic approaches in the context of mobile communication.

\subsection{Mobile communication energy}

Towards the end of the thesis (Chapter 8) we consider energy consumption from both a common and individual energy resource perspective, but for the rest of our work (Chapters 2-7) we analyse the energy consumption of mobile devices from a common energy resource system perspective. The focus is the footprint of the wireless interface of a mobile device, and we propose methods and solutions to quantify and reduce the energy consumption of the entities of the system (e.g., applications).

Figure 1.2 shows a simplified illustration of the system where the total energy consumption of the wireless interface is denoted by $E(N) . E(\{i\})$ represents the energy consumption of an application in isolation (running alone), and $E(S)$ the energy consumption of a set of applications running together. Thus, $E$ represents the energy consumption characteristics of the system. These terms will be used throughout the thesis.

Several aspects impact the energy consumption of a wireless interface, including the hardware chipset of the particular technology (i.e., the set of electronic components in the integrated circuit and its power management) and the data communication created by the commanding software (i.e., entities). The mechanisms employed at lower layers make the energy consumption of data communication highly dependent on the data traffic pattern and the wireless interface. For example, the power management of 802.11 interfaces (WiFi) differs much from the mechanisms employed for cellular interfaces, and thus similar data patterns consume considerably disparate amount of energy due to the operation of the low layer mechanisms. For example, we measured that sending a single packet in $3 \mathrm{G}$ given our network settings consumed 4 joules, whereas sending it in WiFi consumed 0.04 joules for the same mobile device.

When developing energy-efficient solutions for mobile services, it is important to consider that each service can exhibit various interaction patterns 


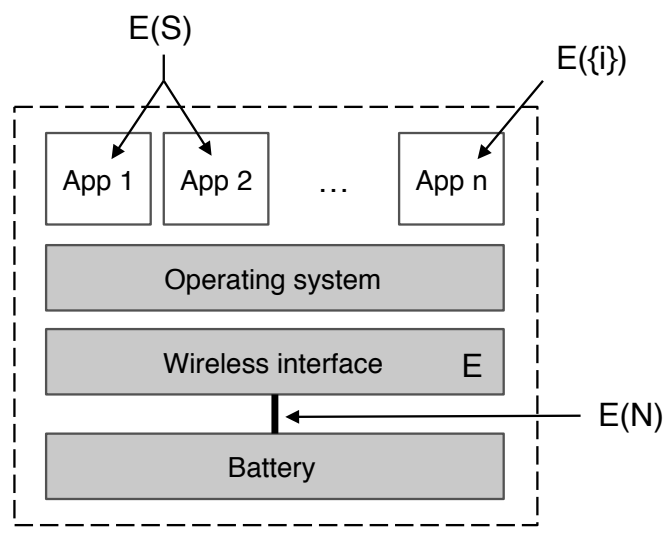

Figure 1.2: Simplified system illustration.

and present distinct requirements in terms of Quality of Service (QoS) such as interactivity, elasticity, tolerance or adaptivity [68]. These requirements limit the solution space and can present different requirements in terms of timeliness, bandwidth or reliability, which are measured using parameters such as jitter, delay, response time, system level data rate or loss [43]. The QoS requirements impact the data communication pattern of applications.

There is so far no requirement from the energy consumption perspective for traffic classes or mobile applications and energy consumption is often considered to be part of the Quality of Experience (QoE). While QoS is an objective measure of the service provided to the end user, QoE is often defined as a purely subjective measure representing the user's perspective of the provided service $[100,232]$. In this thesis, the emphasis is on prioritising energy consumption and quantifying it in order to be able to later consider both the energy and QoS dimension in a meaningful manner.

Two general approaches can be used when developing efficient solutions for mobile communication:

- Generic solution per traffic class: Given the characteristics of a traffic class, the energy consumption can be optimised knowing its QoS requirements (if any). The energy consumption of a set of applications $(E(S))$ generating traffic that falls within the same class can be reduced by a common solution. A traffic characterised for being elastic, best-effort and without any specific QoS requirement (except for the fact that it needs to be sent) introduces flexibility with respect to optimising energy consumption, whereas energy optimisation can be harder for a traffic class with strict real-time requirements.

- Tailor-made solution: Tailor-made energy-efficient solutions are conceivable given the data communication requirements of an application. Even though applications may generate different types of traffic, 
knowledge about the specific usage of these applications (i.e., utilisation characteristics) can suggest possible ways of improving their energy efficiency. For example, the application developers might be willing to balance the inherent performance-energy trade-off at the design stage or provide some levels of adaptivity. Developers can adopt tailor-made solutions to reduce the energy expenditure of an application $(E(\{i\}))$ while maintaining the particular requirements for some application.

In our work we explore techniques that fall into both categories. Nonetheless, improving the energy efficiency of an application or a certain traffic class for some applications (i.e., $E(\{i\})$ and $E(S))$ does not necessarily improve the energy efficiency of the overall system since the wireless interface is shared by many applications. The impact of the interactions among the different applications and the wireless interface is not negligible as every application contributes to the total energy consumption $E(N)$.

In this context, efficiently managing energy requires a detailed understanding of the level of energy consumption in the system. Revealing the contribution of each entity in the system (e.g., application or subprocess) to the total consumption is essential for energy management. Energy accounting is the method that quantifies, analyses and reports the energy consumption of different entities or activities to provide the needed transparency to a system. Unfortunately, determining the contribution of an entity to the total energy consumption of the system or a single component faces the $a p$ portionment problem. This is a hard problem of accounting in general and does not have a single best solution for the general case $[65,129]$. Within our scope, the energy apportionment problem is analogous to determining the contribution of each application to the total energy consumption of the wireless interface.

\subsection{Problem formulation}

The purpose of this thesis is to provide methods and tools to address energy consumption problems in the context of mobile network applications. The research goals are the following:

- Energy awareness: Provide efficient means to support the analysis of communication energy footprint at the user end. The proposed approach should complement physical power measurements, and facilitate the design of energy-efficient solutions.

- Energy saving techniques: Explore and develop methods to reduce the energy consumption of the system. The proposed methods should help to achieve energy efficiency for the entities in the system given knowledge about the activities. The aim is to gain an understanding 
of the advantages and disadvantages of tailor-made and traffic class based solutions.

- Methods to support energy management: Identify and provide methods to perform energy accounting to ultimately support energy management. The proposed means should determine the contribution of the entities in the system to the total energy consumption in order to evaluate their energy efficiency and be illustrated in the context of networking applications.

\subsection{Contribution}

The contributions of this thesis are as follows:

1. A simulation tool to perform communication energy studies at the device end: We propose EnergyBox, a generic energy simulation tool to quantify the energy consumption of wireless interfaces at the device end. EnergyBox captures the influence of the data communication pattern on communication energy at the user end using real data traces as input. The tool provides accurate and repeatable energy consumption estimates for $3 \mathrm{G}$ and $\mathrm{WiFi}$ communication. The strength of EnergyBox is that it allows to modularly configure the $3 \mathrm{G}$ network parameters specified at operator level, the adaptive power save mode for a given $\mathrm{WiFi}$ device, and the device-specific power levels of the operation states for different handheld devices.

2. Analysis of communication energy of mobile applications: Using EnergyBox we quantify and study the communication energy footprint of popular applications which is essential to develop means to reduce the energy efficiency. Our studies pinpoint inefficient communication patterns and mechanisms and quantify the cost of certain features in the context of mobile games and instant messaging applications.

3. Cross-layer background traffic scheduler for 3G: We devise a kernel level energy-efficient solution that schedules data transmissions of a particular traffic class (background traffic) at the user end. The elastic and best-effort background traffic is scheduled based on the current network parameters that impact the $3 \mathrm{G}$ energy consumption. The means to estimate these parameters to be used by the scheduler are also developed. The cost of running the solution is quantified with real application data traffic showing low overhead and significant energy savings.

4. Designing energy-friendly solutions for applications: Given the knowledge of different applications' energy usage, we propose means 
to perform energy-efficient data communication by developing tailormade solutions for two types of applications. In particular, we experimentally compare two application layer protocols for location sharing over cellular networks. The evaluation provides an understanding of the trade-offs between different choices. In addition, the results show that a more compact data format can significantly reduce the energy consumption. Furthermore, in the context of instant messaging, we show that aggregating messages reduces energy consumption.

5. Energy apportionment policies: We identify relevant formal fairness criteria for the energy apportionment problem. In addition, we show that providing incentives to improve the energy efficiency of the system, neglected in previous works, is a relevant purpose of an energy apportionment policy. Our work recognises 8 different energy apportionment policies already proposed in different fields and formalises 3 of them not previously presented mathematically. We also propose 2 alternative novel policies based on cooperative game theory not previously used in energy apportionment. These policies provide a strong fairness notion and a rich incentive structure. Finally, we perform a comparative study of the 10 energy apportionment policies in terms of the formal fairness properties, information requirement and computational complexity. Our work provides guidelines to select an appropriate apportionment policy for a given purpose.

We relate the contributions 2, 3 and 4 to the general practices and solutions presented in section 1.2. Table 1.1 shows the mapping between the general solutions and the contributions.

Table 1.1: Mapping between the contributions and the general solutions.

\begin{tabular}{|l|c|}
\hline General practices & Contribution \\
\hline Use a more efficient alternative for the same task & 2,4 \\
\hline $\begin{array}{l}\text { Adopt holistic solutions to analyse the broad scope } \\
\text { of the problem and consider cross-layer interaction }\end{array}$ & 4 \\
\hline $\begin{array}{l}\text { Optimise the resource usage for the common } \\
\text { utilisation of the system }\end{array}$ & 2,4 \\
\hline $\begin{array}{l}\text { Consider only the minimum desired functionality } \\
\text { and requirements }\end{array}$ & 3,4 \\
\hline Tradeoff some other aspect for energy & 2,3 \\
\hline Match utilisation to the resource characteristics & 3,4 \\
\hline Group multiple activities in time & 3 \\
\hline Spend power to save power & 3 \\
\hline
\end{tabular}

Contribution 1 and 5 enable the quantification of the communication energy of applications. Contribution 2 identifies the usage of a more efficient alternative when quantifying the energy footprint, inefficient traffic patterns and functionalities where the utilisation does not match the resource characteristics. Contribution 3 adopts a cross-layer approach to match the low utilisation of the background traffic to the radio resource characteristics. 
This is done by grouping multiple transmissions and prioritising energy consumption over transmission delay. The cost of the mechanism is quantified showing that it results in total savings. Finally, contribution 4 shows that selecting a more efficient protocol or disabling certain application features improves energy efficiency. Similarly to contribution 3, the proposed solution prioritises energy consumption over transmission delay by grouping transmissions with the knowledge of the application's common utilisation.

\subsection{Research methods}

Computer science is a discipline which has inherited different research methods. For example, theoretical methods (based on logic and mathematics) as well as experimental and simulation methods are combined with an engineering approach of quantification, measurements and comparison. In this section we briefly summarise the employed scientific methods and the main use of each method with respect to the above contributions.

Energy consumption is a result of several complex phenomena including different software and physical artefacts. We follow the experimental method and perform experiments employing physical energy measurements, as well as literature survey of other previous works to understand the main aspects influencing energy consumption.

EnergyBox is the result of modelling the energy consumption phenomenon of mobile devices due to wireless communication among other aspects. We build this model based on our experimental exploration using empirical data to provide a simple, practical and abstract tool that is still accurate. This is validated by comparing the results of the model to the real world phenomenon under the described experimental settings.

To obtain evidence of usability of our tool we apply it in the context of mobile applications. We employ both physical energy measurements and simulation using EnergyBox in order to analyse applications' communication energy with or without our proposed solutions. The proposed energy saving solutions are implemented as proof-of-concept prototypes. This provides understanding of the performance in real settings and the potential practical limitations of our approaches. The experimental methods are presented in each chapter describing in detail the environment as well as the inputs employed.

Finally, we employ theoretical methods using mathematical proofs to analyse whether the considered energy apportionment policies always satisfy certain fairness properties. We leverage game theory, which is defined as the study of mathematical models of conflict and cooperation between intelligent rational decision-makers [158]. 


\subsection{List of publications}

The work presented in this thesis is based on the following publications:

- E. J. Vergara and S. Nadjm-Tehrani. Energy-Aware Cross-Layer Burst Buffering for Wireless Communication. In Proceedings of the 3rd International Conference on Future Energy Systems: Where Energy, Computing and Communication Meet (e-Energy '12). ACM, May 2012.

- E. J. Vergara and S. Nadjm-Tehrani, EnergyBox: A Trace-driven Tool for Data Transmission Energy Consumption Studies, in Proceedings of the International Conference on Energy Efficiency in Large Scale Distributed Systems (EE-LSDS 2013), Lecture Notes in Computer Science, Springer, April 2013.

- E. J. Vergara, M. Prihodko, and S. Nadjm-Tehrani, Mobile Location Sharing: An Energy Consumption Study, in Proceedings of the 4th International Conference on Future Energy Systems: Where Energy, Computing and Communication Meet (e-Energy '13). Poster session, ACM, May 2013.

- E. J. Vergara, J. Sanjuan, and S. Nadjm-Tehrani, Kernel Level Energy-Efficient 3G Background Traffic Shaper for Android Smartphones. In Proceedings of the 9th International Wireless Communications and Mobile Computing Conference (IWCMC). IEEE, July 2013.

- E. J. Vergara, S. Nadjm-Tehrani and M. Prihodko, EnergyBox: Disclosing the Wireless Transmission Energy Cost for Mobile Devices. In Sustainable Computing: Informatics and Systems, Volume 4, Issue 2, Pages 118-135. Elsevier, June 2014.

- E. J. Vergara, S. Andersson and S. Nadjm-Tehrani. When Mice Consume Like Elephants: Instant Messaging Applications. In Proceedings of the 5th International Conference on Future Energy Systems (e-Energy '14). ACM, June 2014.

- M. Almquist, V. Almquist, E. J. Vergara and S. Nadjm-Tehrani. Communication Energy Overhead of Mobile Games. In Proceedings of the 2nd Workshop on Mobile Gaming (MobiGames '15). ACM, May 2015 .

- E. J. Vergara, S. Nadjm-Tehrani and M. Asplund. Sharing the Cost of Lunch: Energy Apportionment Policies. In Proceedings of the 11th ACM International Symposium on QoS and Security for Wireless and Mobile Networks (Q2SWinet '15). ACM, November 2015. 
- E. J. Vergara, S. Nadjm-Tehrani and M. Asplund. Fairness and Incentive Considerations in Energy Apportionment Policies. Submitted for publication to ACM Transactions on Modeling and Performance Evaluation of Computing Systems (TOMPECS). Currently undergoing a post-review revision.

The following publications adopt an individual energy resource perspective of the problem and study a method to reduce the aggregated energy consumption of a set of mobile devices. The publications constitute a complementary approach to the main work and are not included in this thesis:

- E. J. Vergara and S. Nadjm-Tehrani. Watts2Share: Energy-Aware Traffic Consolidation, In Proceedings of the IEEE Green Computing and Communications (GreenCom). IEEE, August 2013.

- A. Bianzino, M. Asplund, E. J. Vergara, and S. Nadjm-Tehrani. Cooperative Proxies: Optimally Trading Energy and Quality of Service in Mobile Devices. Computer Networks, Volume 75, Part A, Pages 297-312. Elsevier, December 2014.

The following publications are peripheral to the work presented in this thesis and served as motivation for the work described in the thesis:

- E. J. Vergara, S. Nadjm-Tehrani, M. Asplund and U. Zurutuza. Resource Footprint of a Manycast Protocol Implementation on Multiple Mobile Platforms, In Proceedings of the 5th IEEE International Conference on Next Generation Mobile Applications, Services and Technologies (NGMAST '11). IEEE, September 2011.

- M. Asplund, A. Thomasson, E. J. Vergara, and S. Nadjm-Tehrani. Software-related energy footprint of a wireless broadband module. In Proceedings of the 9th ACM International Symposium on Mobility Management and Wireless Access (MobiWac '11). ACM, November 2011.

Finally, the following book chapter was composed by individual contributions by several researchers in the area of green wireless networking in order to provide an overview of the field:

- V. Bernardo, T. Braun, M. Curado, M. Fiedler, D. Hock, T. Hossmann, K. A. Hummel, P. Hurni, S. Ickin, A. Jamakovic-Kapic, S. Nadjm-Tehrani, T. A. Trinh, E. J. Vergara, F. Wamser and T. Zinner. Green Wireless Energy Efficiency in Wireless Networks. In Large-Scale Distributed Systems and Energy Efficiency, pages 81-130. John Wiley \& Sons, Inc., 2015. 


\subsection{Thesis outline}

The thesis is organised as follows:

Chapter 2 - Background provides the needed background to understand the rest of the thesis. In particular, the energy consumption problem in mobile communication is explicitly illustrated using real instant messaging applications, the technological background regarding $3 \mathrm{G}$ and $\mathrm{WiFi}$ is provided, and an overview of the research efforts and directions is described.

Chapter 3 - EnergyBox presents the communication energy simulation tool for $3 \mathrm{G}$ and $\mathrm{WiFi}$. The chapter describes the modelling of the lower layer mechanisms and the validation against physical measurements in order to provide accurate energy consumption studies.

Chapter 4 - Communication Energy of Games quantifies and analyses the individual communication energy footprint of a selection of 20 popular mobile games. This chapter illustrates that EnergyBox supports and facilitates energy studies.

Chapter 5 - Cross-layer Burst-Buffering describes and evaluates the energy saving technique developed for the background traffic class originated from different applications.

Chapter 6 - Energy-Efficient Location-Sharing focuses on studying the impact of the choice of protocols on the energy consumption of location sharing applications using EnergyBox.

Chapter 7 - Energy-Efficient Instant Messaging describes the work done in the context of instant messaging applications.

Chapter 8 - Energy Apportionment Policies studies different methods to prescribe the energy contribution of an entity to the total consumption of the system.

Chapter 9 - Related Works presents a survey of the related works providing a taxonomy for the main work in the area. The reasons for placing the taxonomy at the end are that Chapter 9 is not fundamental for understanding the thesis, our approaches and others works are more suitably compared once they are described, and the taxonomy includes works that are orthogonal to the main focus of the thesis but still within the wireless energy efficiency scope.

Chapter 10 - Conclusion and Future Work concludes the work presented in the thesis and describes directions for future work.

Finally, Figure 1.3 illustrates the focus of each chapter as well as its mapping to the contributions of the thesis. Chapter 3 provides the means 
System illustration

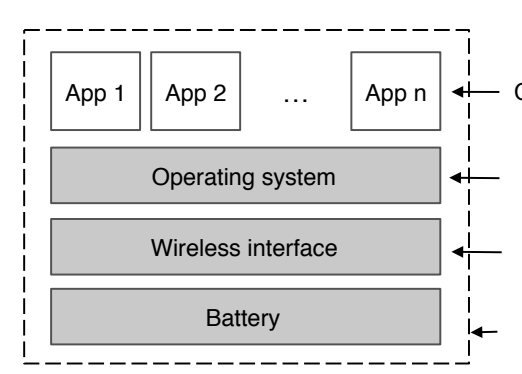

Thesis contributions and structure

\begin{tabular}{c|c|c} 
Chapters & Contribution & Focus \\
Ch. 4, 6, 7 & 2 and 4 & $\mathrm{E}(\{\mathrm{i}\})$ \\
Ch. 5 & 3 & $\mathrm{E}(\mathrm{S})$ \\
Ch. 3 & 1 & $\mathrm{E}$ \\
Ch. 8 & 5 & $\mathrm{E}(\mathrm{N})$
\end{tabular}

Figure 1.3: Simplified system illustration and the mapping to the contributions, chapters and the focus of each chapter.

to estimate the energy consumption of the wireless interface $E$. Chapters 4,6 and 7 focus on quantifying and providing means to reduce $E(\{i\})$. The solution presented in Chapter 5 reduces the energy consumption of $E(S)$, and finally Chapter 8 studies methods to perform the apportionment of $E(N)$ among the entities in the system. 
"The true delight is in the finding out rather than in the knowing."

Isaac Asimov

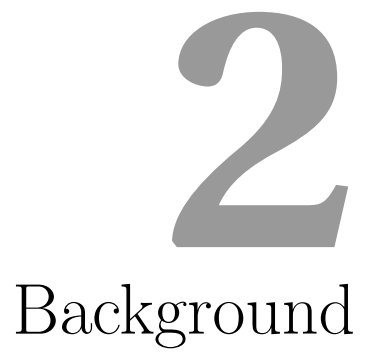

This chapter provides the needed background for an understanding of the contributions presented in this thesis. In section 2.1, we illustrate the fact that some applications can unjustifiably consume much more energy than similar ones due to their communication pattern. Section 2.2 provides a background for the energy consumption of the technologies used to implement the concepts developed in this thesis ( $3 \mathrm{G}$ and $\mathrm{WiFi})$. Finally, a brief overview of the research efforts towards lowering the energy consumption of wireless communication at the user end is described in section 2.3 .

\subsection{Application communication energy}

There are several reasons why different applications can consume different amounts of energy. For example, they might provide different features or implement different communication mechanisms. However, the fact that functionally similar applications can consume completely different amounts of energy is unjustifiable from a user's QoE perspective. This issue is explored and described through an illustrative example concerning instant messaging applications. The charts in this section are adapted from the Bachelor thesis by Simon Andersson [12] that was supervised by the author.

We select 6 of the most popular instant messaging applications from the Android market as a test set. Since the applications are products and their code is not available, we consider them as black boxes. The same simple short conversation is created using two different smartphones connected via $3 \mathrm{G}$ using the different applications. The 2 minute conversation is shown in Table 2.1. The test is repeated 5 times and we collect the packet traces in 
Table 2.1: Short conversation.

\begin{tabular}{|rcl|}
\hline $\begin{array}{r}\text { Timestamp } \\
\text { (min:sec) }\end{array}$ & $\begin{array}{c}\text { User } \\
\text { number }\end{array}$ & $\begin{array}{l}\text { Message } \\
\text { sent }\end{array}$ \\
\hline $0: 0$ & user1 & Hello \\
$0: 15$ & user2 & Hi! \\
$0: 32$ & user1 & How are you? \\
$1: 01$ & user2 & I am great, thank you! \\
$1: 14$ & user2 & You? \\
$1: 33$ & user1 & I'm good thanks. \\
$1: 48$ & user1 & Bye \\
$1: 56$ & user2 & Bye \\
\hline
\end{tabular}

the smartphone of user 1. The energy consumption for each application is calculated using EnergyBox, our tool that is described later in Chapter 3.

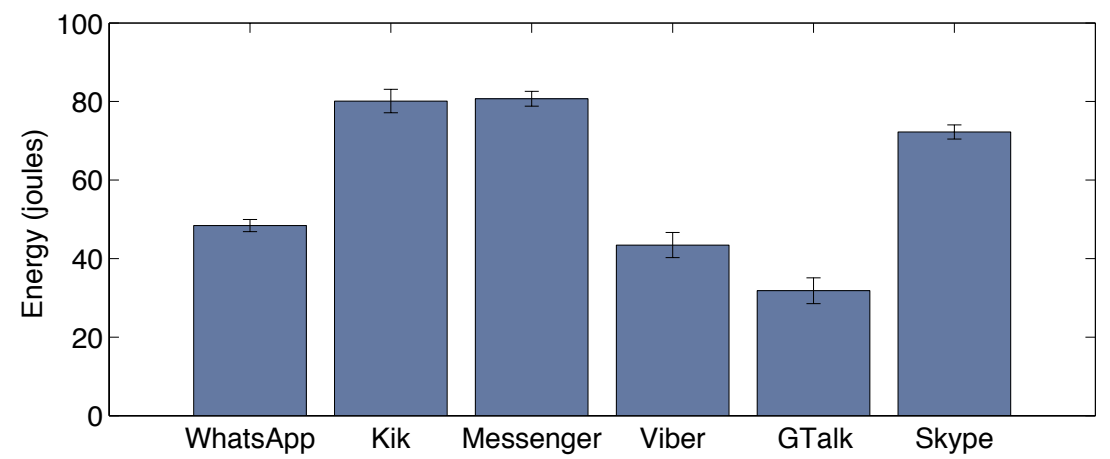

Figure 2.1: Communication energy and standard deviation of different Instant Messaging applications for the short conversation.

Fig. 2.1 shows a great diversity regarding the amount of energy spent by the different applications when exchanging the short conversation. The most consuming application (Messenger) consumes 153\% more energy than the least consuming one (GTalk) to transmit the same conversation. This short conversation using Messenger consumes $0.3 \%$ of a commonly used mobile phone battery type (2000 mAh at $3.7 \mathrm{~V})$. The difference from using the least energy-efficient application could substantially shorten the battery lifetime of a device by a factor of 2.5 reducing the QoE of the user.

The data traffic pattern characterises the transmissions performed by an application. Fig. 2.2 shows the amount of data sent, the number of packets and the empirical cumulative distribution functions (CDF) of packet size and inter-packet interval during the experiments. These parameters are often used to describe the transmission characteristics. For clarity, we select only 3 of the 6 applications for the CDF graphs.

In general, one can observe significant diversity in the different parame- 
ters for different applications. WhatsApp and GTalk employ few and small packets, whereas Messenger sends a lot of data and $10 \%$ of the packets are greater than 1000 bytes. The high inter-packet interval of GTalk describes sparse data transmissions, and therefore longer idle periods between transmissions than WhatsApp and Messenger. Even though Kik sends much less data than Messenger, it results in similar energy consumption.

The data encoding, protocol of choice at different layers, added features or functionalities can highly influence the data pattern, and thereby impact the energy consumption. The user interaction becomes a decisive factor when considering the data pattern as well.
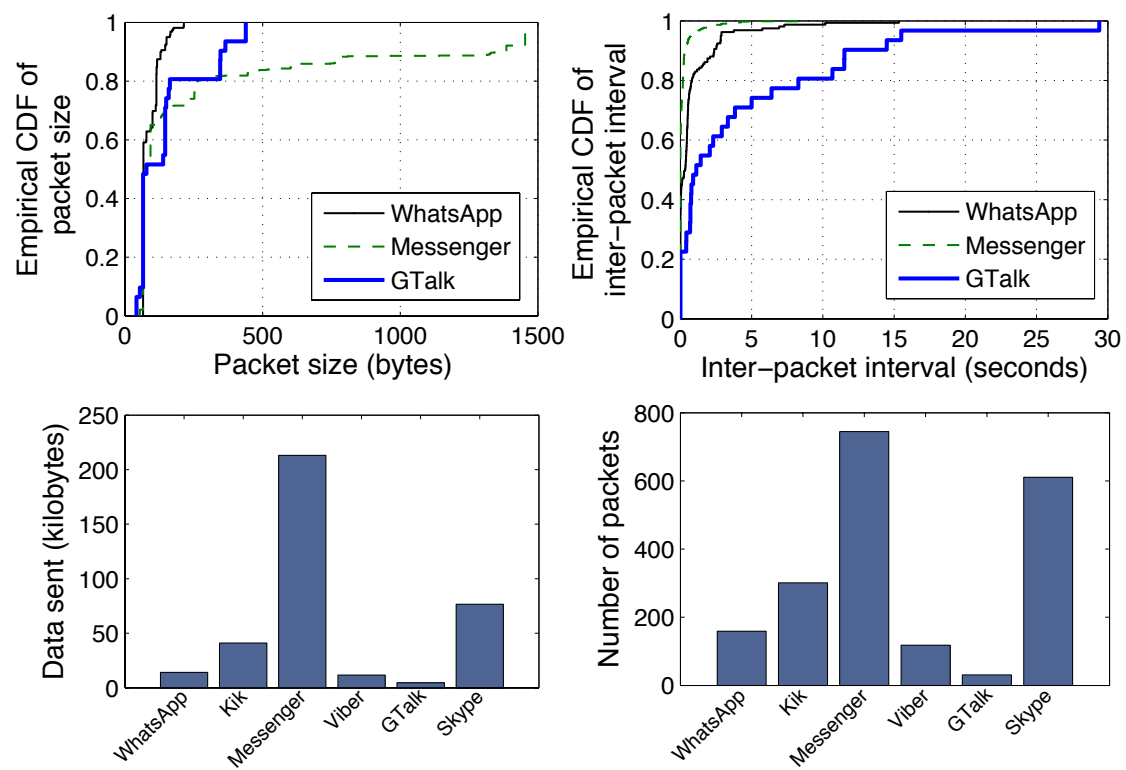

Figure 2.2: Data pattern characteristics of the different Instant Messaging applications for the short conversation.

The above measurements have illustrated the need to identify the data pattern of the application (or other system software) and its impact on the energy consumption.

\subsection{Technological background}

This section provides a background for communication energy footprint of $3 \mathrm{G}$ and $\mathrm{WiFi}$ at the user end through descriptive measurements that show the main factors affecting the data communication energy. 


\subsubsection{G energy consumption}

The Radio Network Controller (RNC) is a key element in the UMTS ${ }^{1}$ Terrestrial Radio Access Network (UTRAN). It is responsible for the radio resource management and also manages the Node Bs (also known as base transceiver station), to which the user equipment (UE) connects via radio physical channel. The energy consumption of the UE in 3G is mostly influenced by the Radio Resource Control (RRC) and the Radio Link Control (RLC) protocols, which are defined in the UMTS WCDMA ${ }^{2}$ protocol stack.

According to the RRC, the UE implements a state machine. Fig. 2.3 shows the RRC state machine as depicted by one of the major providers of telecommunication technology. The states imply different performance (response time and data rate) and power consumption based on the kind of physical channels that the UE is using. The RRC state machine has been fairly unchanged between the 3GPP Rel. 99 to Rel. 7 (TS 25.331 [1]). The $\mathrm{RRC}$ state machine is also described in the TS 36.331 specification [2].

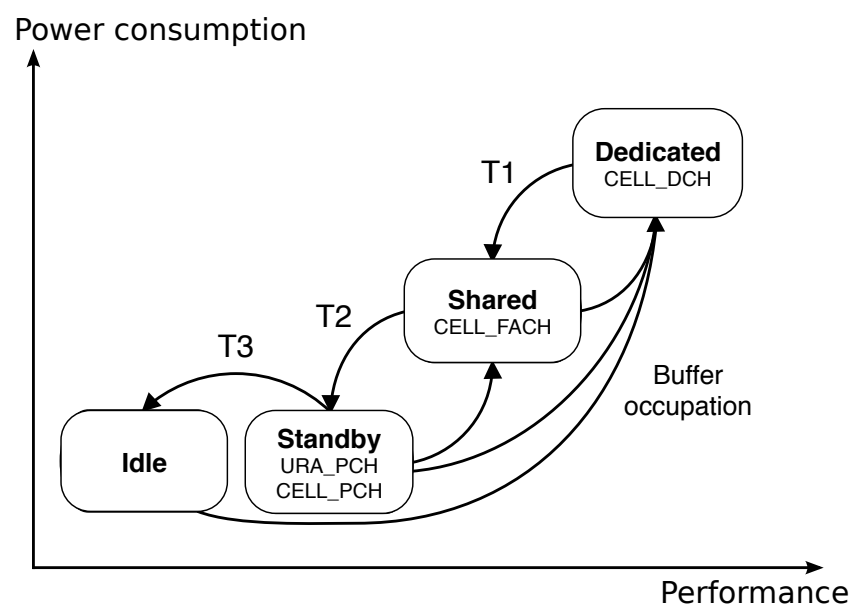

Figure 2.3: RRC state machine as depicted in Ericsson's labs [72].

RRC States: There are two basic operational modes: Idle and Connected. In the connected states, the UE has already established a RRC connection.

In the Dedicated state (CELL_DCH), a dedicated physical channel is allocated for the terminal in both uplink and downlink providing higher data rates. The terminal has access to dedicated uplink or downlink transport channels, shared transport channels and a combination of them. In the Shared state, i.e., Forward Access Channel (CELL_FACH), the terminal

\footnotetext{
${ }^{1}$ Universal Mobile Telecommunications System

${ }^{2}$ Wideband Code Division Multiple Access
} 
is assigned a default common or shared transport channel in the uplink (e.g., the Random Access Channel (RACH)) and monitors the downlink continuously. The UE can transmit small data packets at lower data rates. While in CELL_DCH and CELL_FACH the UE remains connected to the RNC.

The UE is in the Idle state when there is no network activity. It is not connected but it still can check if there is any downlink packet available. Denoted as Standby in Fig. 2.3, the $3 \mathrm{G}$ standard also describes two optional states where the UE maintains a connection to the RNC and the energy consumption is similar to the Idle state: the Paging Channel (CELL_PCH) and UTRAN ${ }^{1}$ Registration Area Paging Channel (URA_PCH). These two states allow the UE to switch faster to higher states.

In CELL_PCH no resources are allocated for data transmission and the terminal can use Discontinuous Reception (DRX) to check if there is any downlink packet. DRX reduces the energy consumption by receiving one paging occasion per DRX cycle.

URA_PCH is similar to CELL_PCH but it has some performance improvements regarding mobility. Note that some operators do not implement the optional states. In most of our work, the UE was connected to an operator (TeliaSonera) that implements the URA_PCH state. For the rest of the thesis we will refer to the different states as DCH, FACH, PCH and Idle.

State transitions: State transitions on the UE occur based on traffic volume and inactivity timers controlled by the RNC. Statically set inactivity timers control the state transitions DCH-FACH, FACH-PCH and PCH-Idle, denoted by $T_{1}, T_{2}$ and $T_{3}$ in Fig. 2.3 respectively. For example, when the UE is in the DCH state for $T_{1}$ seconds without any or small data transmission, the RNC releases the dedicated channel and switches the UE to FACH by means of the RRC protocol. Some networks implement a low activity mechanism in DCH to release the transport channel and move to FACH when there is low traffic [102].

The RRC uses information from the RLC protocol [203] in order to report the observed traffic volume to the network. For example, in FACH, the UE reports to the RNC the observed traffic volume based on data buffer status. This helps the RNC to re-evaluate the allocation of resources. The RLC data buffer is used to trigger state transitions. When the content of the data buffer exceeds a certain threshold, the corresponding signalling is performed before switching the state. There are 4 RLC buffer thresholds, 2 uplink and 2 downlink. These buffers are cleared out when the data is transmitted.

Fig. 2.4 shows the power consumption levels of the states experienced at one location using the operator TeliaSonera in Sweden when the UE sends a packet burst. All the physical measurements are performed with our measurement setup described in Appendix B. The 3G module stays in $\mathrm{PCH}$ state until there is a transition to FACH $(1 \mathrm{~s}$ in Fig. 2.4). There is no

\footnotetext{
${ }^{1}$ UMTS Terrestrial Radio Access Network
} 


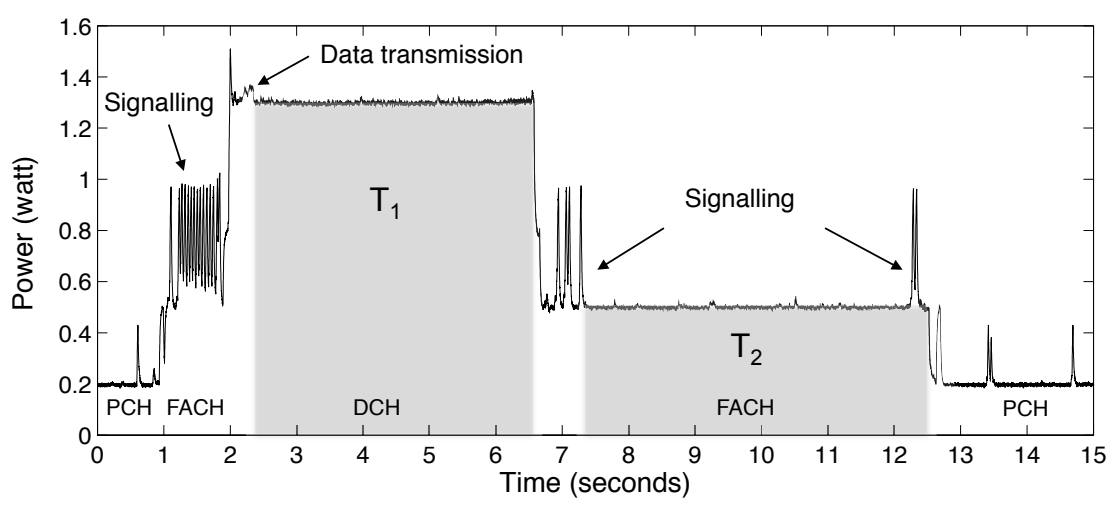

Figure 2.4: Example 3G power profile for the operator TeliaSonera. The RRC states are annotated.

need to send signalling to the network for transitioning to FACH [102].

Given the high amount of data that the UE will send in the example, it needs to be allocated within DCH. This state transition is described in more detail as an example. The UE sends a Cell update in RACH to the RNC, which responds with a Cell update confirm in FACH. Next, the UE sends the UTRAN mobility information confirm message and a measurement report. Then the radio link is setup, and the RNC sends a Radio bearer reconfiguration to move the $\mathrm{UE}$ to $\mathrm{DCH}$. The necessary signalling for the state transition takes more than 1 second (from $1 \mathrm{~s}$ in Fig. 2.4), and it has some extra energy cost for exchanging the messages.

Once the UE is on DCH the data is downloaded. The transition to FACH occurs when all dedicated channels have been released $(7 \mathrm{~s}$ in Fig. 2.4). The power consumption in the DCH state is around $1.3 \mathrm{~W}$, higher than the FACH state (around $0.5 \mathrm{~W}$ ) and $\mathrm{PCH}$ state $(0.2 \mathrm{~W})$.

Table 2.2: State transitions and triggering packet sizes for TeliaSonera measured in July 2012.

\begin{tabular}{|c|c|c|}
\hline $\begin{array}{c}\text { State } \\
\text { Transition }\end{array}$ & $\begin{array}{c}\text { Uplink threshold } \\
\text { (bytes) }\end{array}$ & $\begin{array}{c}\text { Downlink threshold } \\
\text { (bytes) }\end{array}$ \\
\hline PCH-DCH & $513-542$ & $524-558$ \\
\hline FACH-DCH & 294 & 515 \\
\hline PCH-FACH & \multicolumn{2}{|c|}{ Always triggered } \\
\hline
\end{tabular}

We measured the uplink and downlink thresholds that trigger the state transitions shown in Table 2.2. They were measured by sending UDP packets of different sizes and observing the live power trace using our measurement 
setup. Note that the PCH-DCH transition is more likely to happen when the triggering packet is closer to the upper bound, i.e., transmitting 513 bytes uplink might not always lead to a state transition. Our initial measurements were carried out over an interval of 5 months, and 3 months into the period the operator settings were changed so that the value of the uplink RLC data buffer threshold that triggers $\mathrm{PCH}-\mathrm{DCH}$ transition was incremented from the value of Table 2.2 to around 900 bytes ( 875 - 1000 bytes). The same value was measured in February 2015.

It can be noticed that after downloading the data, the UE continues in the DCH state until the inactivity timer $T_{1}$ has expired causing an energy consumption overhead. A similar overhead is caused in FACH due to $T_{2}$. Inactivity timers create energy overheads known as energy tails due to the UE remaining in high consuming state while not transmitting anything [24]. These energy overheads are shown as shaded areas in Fig. 2.4 for the DCH and FACH states. To reduce these energy overheads, the fast dormancy mechanism of the $3 \mathrm{GPP}$ Release 8 standard allows the UE to signal the RNC the desire to switch to the lowest power state by sending a Signalling Connection Release Indication before the inactivity timeout.

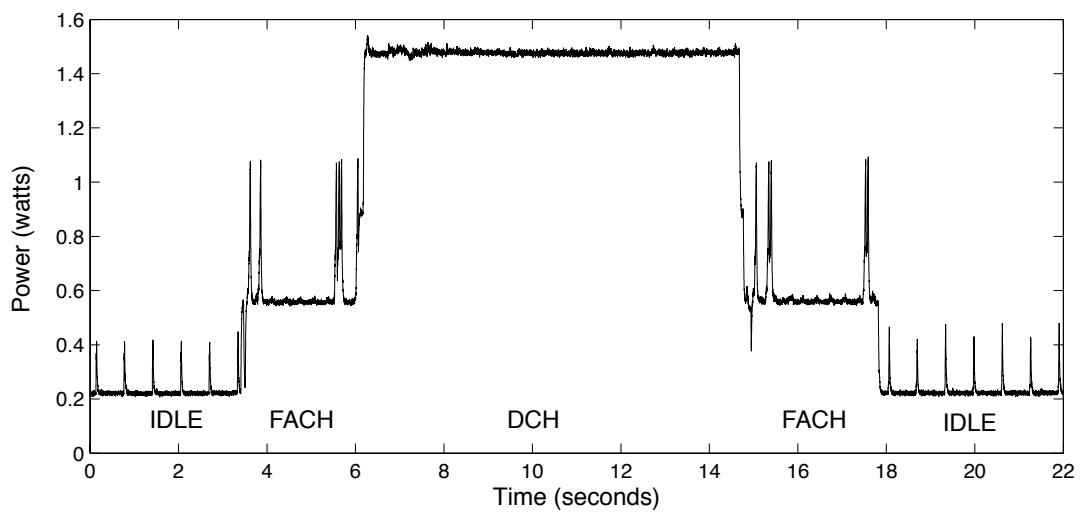

Figure 2.5: Example 3G power profile for operator 3.

The inactivity timers and RLC buffer thresholds vary per operator influencing the energy consumption of the UE. Fig. 2.5 shows the power profile of the operator Hi3g Access AB under the commercial mark 3 measured at our location in Sweden. The power profile is significantly different from Fig. 2.4 since the states implemented by the operator and the configuration parameters differ, e.g., the IDLE state is employed instead of $\mathrm{PCH}$ and the inactivity timers are different.

Fig. 2.6 illustrates the fact that the data traffic pattern highly influences energy consumption of the UE. The UE transitions from $\mathrm{PCH}$ to $\mathrm{DCH}$ (Fig. 2.6 at $3 \mathrm{~s}$ ) since the data burst to be received exceeds the RLC buffer threshold, whereas it transitions and stays in FACH (Fig. 2.6 at 20 and $28 \mathrm{~s}$ ) 
when the thresholds are not exceeded. Fig. 2.6 also illustrates how sporadic transmissions restart the inactivity timers increasing the overhead. Sporadic transmissions of small packets can lead to high energy consumption. As an illustrative example, we sent a 600 bytes UDP packet every 3 seconds during 1 minute which led to a similar consumption to a Skype voice call (average of $19 \mathrm{kB} / \mathrm{s}$ ): 90 and $92 \mathrm{~J}$ respectively. This shows that the $3 \mathrm{G}$ interface is not energy proportional, and thus we see that the energy tails and the above mechanisms make the energy consumption dependent on the traffic pattern and operator settings in a complex way.
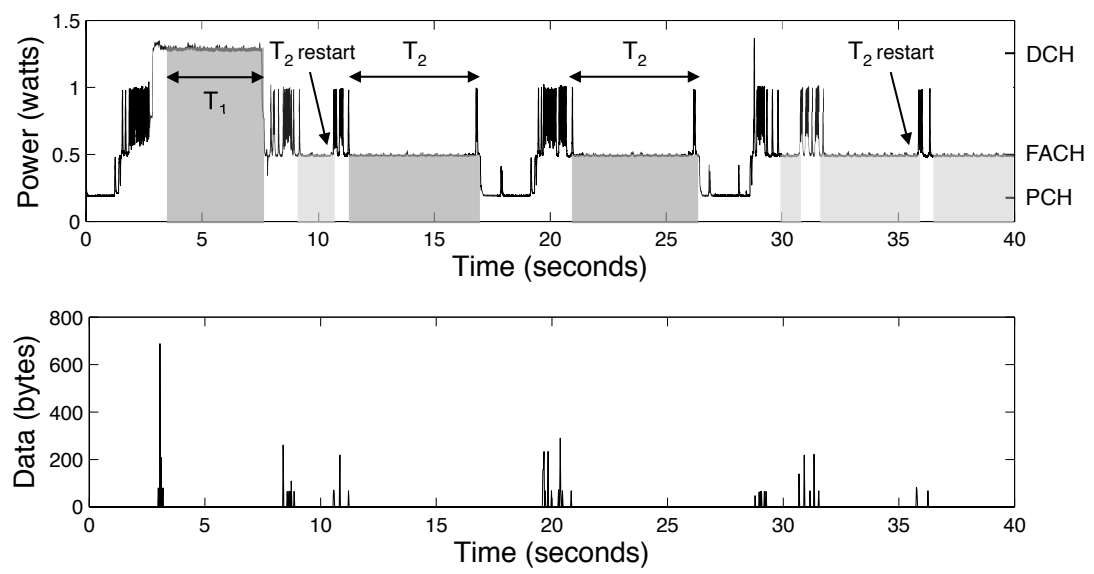

Figure 2.6: Example power profile and data communication for a Skype chat session and the TeliaSonera operator.

To sum up, the statically set inactivity timers and the RLC buffer thresholds at the operator end make the data pattern to drastically impact the energy consumption at the user end.

\subsubsection{WiFi energy consumption}

The communication energy consumption for WiFi is mostly influenced at the driver level in the WiFi station (the client handset) depending on the power save mode used. The station is in the Constantly Awake Mode (CAM) when it has the power-saving features disabled experiencing the best performance.

The IEEE 802.11 standard defines a Power Save Mode (PSM), which allows the stations to switch to low power mode during predefined periods of time when not transferring any data. The access point (AP) buffers downlink frames for the clients and the clients wake up periodically (at multiples of the beacon interval). The clients send a Power Save Poll (PS-Poll) message to the AP to receive each buffered frame. Recent smartphones implement a mechanism named Adaptive PSM to overcome the overhead and latency drawback of using this PS-Poll mechanism [124]. The client 

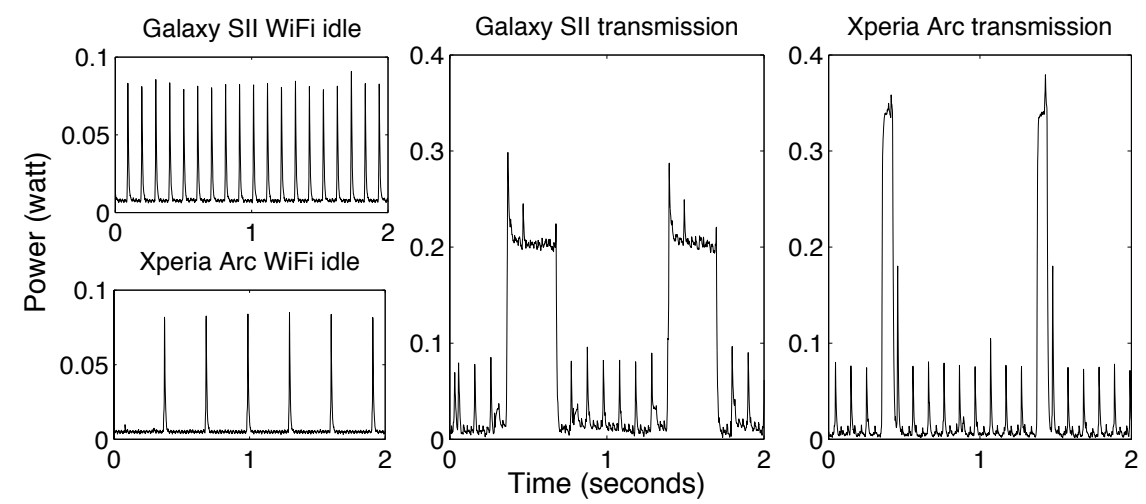

Figure 2.7: Power levels of adaptive PSM for Samsung Galaxy SII and Sony Ericsson Xperia Arc.

switches between the CAM and PSM modes based on heuristics (e.g., number of packets, traffic inactivity period or screen on/off). The client uses the power management field in null data frames to inform the AP about its current mode.

Fig. 2.7 shows an adaptive PSM implementation in the Samsung Galaxy SII and Sony Ericsson Xperia Arc smartphones, where the station moves to CAM for sending the traffic, and switches back from CAM to PSM after a predefined inactivity timeout $(\delta)$ without packet transmission. This $\delta$ timeout creates an energy tail in the similar way as in 3G. Repeated measurements have shown $\delta$ to be around 220 and $70 \mathrm{~ms}$ for the Samsung Galaxy SII and Xperia Arc respectively, much shorter than in 3G. Previous generation devices implement longer $\delta$ timeouts (e.g., 1.5 seconds for HTC Magic) $[140,193,213]$. Moreover, some drivers also implement packets per second thresholds ( $U p$ and Down) that trigger PSM-CAM and CAM-PSM transitions respectively [192].

While in the same state, the station consumes more power when the data rate increases. In order to show the impact of data rate on transmission power and $\delta$, we create an uplink data stream varying inter-packet interval and packet size using the Xperia Arc. Fig. 2.8 shows that for higher data rates (i.e., short inter-packet interval), the average power level increases substantially. When the inter-packet interval is increased to $70 \mathrm{~ms}$, the station switches back to PSM since the inter-packet interval is greater than $\delta$. This drops the average power level. To sum up, adaptive PSM leads to a high impact by the data pattern on the energy consumption of a WiFi station. 


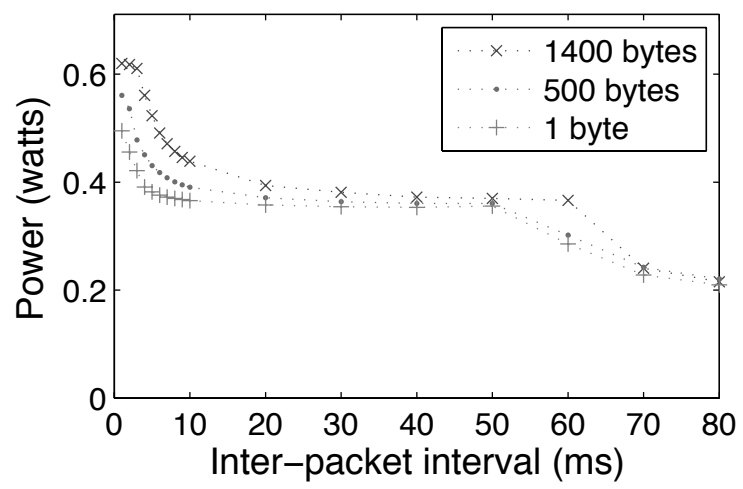

Figure 2.8: Power levels of adaptive PSM for the Sony Ericsson Xperia Arc.

\subsection{Overview of related works}

This section provides an overview of the works in the context of communication energy at the user end. We describe the notions behind the different categories employed to describe the main body of work and its focus. While a great part of the literature regarding the energy efficiency of mobile devices focuses on individual components (e.g., wireless interfaces or other components), we also include an interesting body of work considering a broader perspective than communication energy due to its relevance.

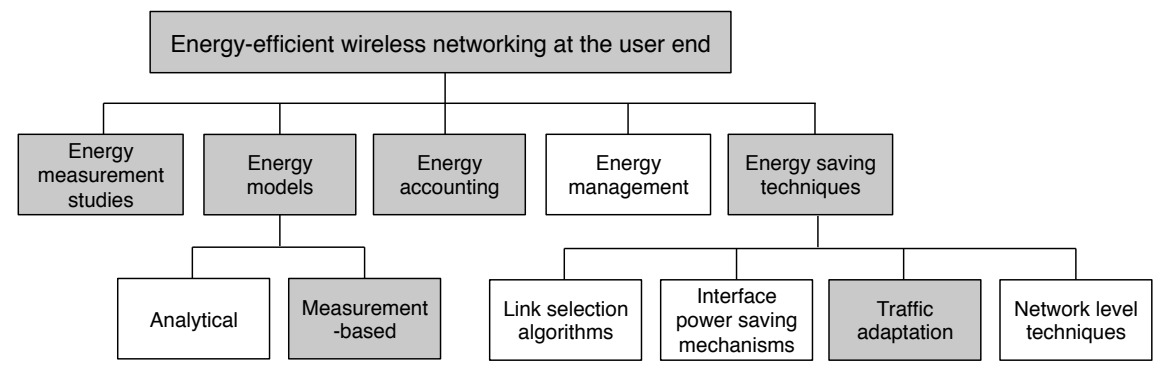

Figure 2.9: Taxonomy of wireless communication energy at the user end.

Fig. 2.9 shows the general categorisation of the works. The contributions of this thesis are within the shaded categories. A complete survey of the works that fall within each category can be found in Chapter 9. A brief overview of the categories is provided next.

Energy measurement studies typically aim at revealing inefficiencies (e.g., profiling), discovering and understanding the footprint characteristics of wireless interfaces. The purpose is often to provide guidelines for a more energy-efficient design and operation. Chapters 4, 6 and 7 analyse the com- 
munication energy footprint of mobile applications and provide guidelines and solutions to reduce their energy consumption.

Energy models focus on characterising energy consumption for the general operation of wireless interfaces or specific mechanisms. Our contribution in Chapter 3 is an energy model based on physical measurements.

Energy accounting is the procedure of quantifying, analysing and reporting the energy consumption of different entities or activities of interest in the system. These works aim at attributing a part of the total energy consumption of a system (or subsystem) to the responsible entities. Chapter 8 contributes in this category.

Energy management is the procedure to allocate energy consumption to competing entities in the system. Energy accounting plays a vital role for energy management.

Energy saving techniques are categorised in subgroups based on their aim and the means used to reduce energy consumption.

- Link selection algorithms focus on algorithms to dynamically select the best available link at a given point in time. These algorithms are based on the fact that different wireless interfaces have different energy consumption characteristics.

- Interface power saving mechanisms are techniques implemented in the lower layers of the interfaces to improve the operation specified by the standard of particular interfaces (e.g., WiFi PSM), optimising the operational parameters or adapting the operation based on the utilisation knowledge (e.g., high loads or a specific type of traffic).

- Traffic adaptation techniques consider the power saving mechanisms as given, and adapt the traffic (i.e., the utilisation) to reduce the energy consumption. Since the data pattern greatly influences the energy consumption, these techniques attempt to achieve energy-efficient patterns by, for example, reducing the amount of data to be sent or batching transmissions. Chapters 5 and 7 propose traffic adaptation techniques.

- Network level techniques consider a set of devices whereas previous solutions were focused on a single mobile device. These works aim at organising the energy expenditure of a group of nodes so that it is minimised by relaying data traffic.

To sum up, the contributions of this thesis are within energy measurement studies, traffic adaptation energy saving techniques, measurementbased energy models and energy accounting. 
"Measurement is the first step that leads to control and eventually to improvement. If you can't measure something, you can't understand it. If you can't understand it, you can't control it. If you can't control it, you can't improve it."

H. James Harrington

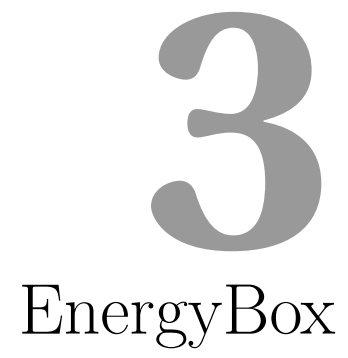

Actual data communication patterns depend on application operation, which is in turn influenced by application design choices (e.g., developer choices for application mechanisms, communication protocols or data format) and the user-device interaction $[75,175]$. The previous chapter has shown how the data communication pattern drastically influences the energy consumption of a mobile device. Ergo, the data pattern becomes an important factor in our work when analysing energy consumption.

We argue that reducing the energy consumption of wireless communication begins by being aware of the energy consumption characteristics of wireless interfaces (characterised by the term $E$ ) for different technologies. While physical power measurements can undoubtedly support the development of energy-efficient solutions, performing these measurements is a non trivial task which requires specific knowledge.

We observe that due to the high cost of the measurement solutions and time limitations (e.g., time-to-market of applications), third party software developers cannot afford to invest in these solutions, and applications and system software are not designed or tested with energy consumption in mind. Besides, physical energy measurement studies are laborious and timeconsuming. Thus, solutions are usually tested with synthetic data traces and little variation of factors such as network settings, and hardware dependence. As a complement to physical measurements, we believe that a modular approach to carrying out flexible and efficient energy studies which isolates the communication energy is essential.

We propose EnergyBox, a tool that enables accurate studies of communication energy consumption at the user end, using real traffic traces 
as input. It focuses on the most widespread wireless technologies (3G and $\mathrm{WiFi}$ ) and simulates application data communication energy footprint at the user device $(E)$. EnergyBox simulates the underlying states of operation of the wireless interfaces. The hardware dependency is overcome by using parametrised device specific power levels. For a given data trace, EnergyBox automatically outputs the operation states over time, so that when combined with device specific power levels energy consumption can be estimated. The general idea of EnergyBox is shown in Fig. 3.1.

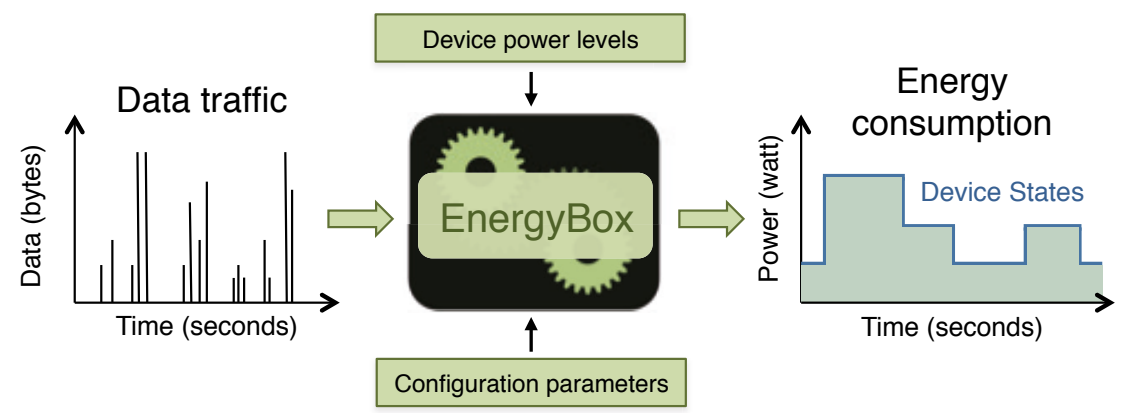

Figure 3.1: Overview of EnergyBox function.

The data traces can be directly captured on different devices or synthetically created in order to study the impact of different communication patterns under different configurations for the given wireless technologies. EnergyBox assists researchers and application developers to immediately estimate energy consumption of data communication for a diverse range of test cases. The scenario is set up using different data traces, device power levels and network settings.

The tool accepts the operator-configured parameters at the radio layer, such as inactivity timers, the data buffer thresholds and a low activity mechanism used for state transition decisions in $3 \mathrm{G}$ communication. For WiFi, it incorporates the adaptive power save mode commonly used as the power saving mechanism in mobile devices.

Using nine different applications with different data patterns the versatility and accuracy of the tool was evaluated. The evaluation was carried out for a popular smartphone in the WiFi setting, a specific mobile broadband module for the $3 \mathrm{G}$ setting, and within the operating environment of a major mobile operator in Sweden. A comparison with real power traces indicates that EnergyBox is a valuable tool for repeatable and convenient studies. It exhibits an accuracy of $94-99 \%$ for $3 \mathrm{G}$, and $93-99 \%$ for WiFi compared against physical measurements given the studied applications' traces.

This chapter is structured as follows: Section 3.1 motivates the need for EnergyBox analysing the available solutions in the industry. Section 3.2 describes the design of the $3 \mathrm{G}$ and $\mathrm{WiFi}$ models included in the EnergyBox and section 3.3 evaluates the accuracy of the EnergyBox compared to 
physically measured energy consumption. Finally, section 3.5 discusses the results and the current limitations of EnergyBox.

\subsection{Motivation}

Several commercial vendors provide hardware and software to analyse the energy consumption in mobile devices.

Measurement platforms for mobile devices typically intercept battery terminals to measure power consumption. These provide aggregated power measurements of network interfaces and other components (e.g., CPU, screen or sensors). The devices used to acquire the measurements range from lab bench multimeters [6], oscilloscopes [235], direct current sources with battery emulation [5], to USB data acquisition units [160], similar to the tools employed in our physical measurement setup presented in Appendix B or in other studies $[208,213]$. The Monsoon Power Monitor is a widely used power measurement device in the research community [154]. These provide high measurement accuracy, but they limit the studies to a fixed environment and measure only aggregated power from all the components of a device.

Advanced testing environments such as the CMW500 from Rhode and Schwarz [210] are used in production testing of wireless devices. The CMW500 radio communication tester emulates access points and settings of different layers of various networks (e.g., Global System for Mobile communication (GSM) or WCDMA) allowing multiple test cases ranging from power and spectrum measurements to protocol stack analysis. While this type of high end solution is great, the high monetary cost and the required training makes them a less suitable choice for mobile application developers.

Vendor-specific development platforms such as the ones provided by Qualcomm [200] use dedicated power measurement circuitry supporting a direct and convenient approach to perform accurate power measurements. The Trepn Profiler tool [201] that uses their own specific hardware is only available for a particular family of processors (Snapdragon), and thus the solution is platform-dependent.

In addition, smart battery interfaces providing current values are available in some devices. However, these measure the aggregated current draw from all the device components. The Nokia Energy Profiler [166] or the CurrentWidget for Android [58] are examples of applications exploiting these interfaces. Dong et al. [64] state that the inaccuracy of the instant battery interface reading is usually high.

Finally, even though there exist some application testing solutions [13, 162] that aim at optimising application performance employing usage metrics (e.g., database, CPU and memory), they typically lack the energy efficiency assessment aspects.

Therefore, as a complement to the above physical measurements and software solutions, we believe that a modular approach to carrying out flexible and efficient energy studies to isolate the communication energy is essential. 


\subsection{EnergyBox design}

EnergyBox is a tool for characterising the data communication energy through trace-based iterative packet-driven simulation. The usage of real data traces means that the estimated energy is realistic, and reflects the impact of the real throughput and delay in the network for that data pattern. EnergyBox also accepts synthetic data traces as input, creating repeatable tests for a given purpose.

Given a packet trace and configuration parameters, EnergyBox outputs the device states $S(t)$ over time. The total energy consumption is calculated by associating these states with state-based power levels for a given device and integrating them over time.

Device-specific power level values can be obtained through measurement platforms as the one described in Appendix B or earlier data $[24,193,208$, 256]. These parametrised power levels abstract the hardware dependency and allow focusing on an application footprint on a given device. EnergyBox also takes as input the $3 \mathrm{G}$ network parameters specified at operator level and the adaptive PSM mechanism specified at the station driver for WiFi. In the following subsections we describe the detailed design of the two modes of operation in EnergyBox. The procedure for collection and instantiation of the input parameters is described in section 3.3.3. The parameters are summarised in Tables 3.1 and 3.2 for $3 \mathrm{G}$ and $\mathrm{WiFi}$ respectively.

\subsubsection{G model}

The energy consumption of $3 \mathrm{G}$ is captured by a parametrised finite state machine that simulates the inactivity timers, the RLC buffers and a low activity mechanism in a packet-driven manner. Fig. 3.2 (a) shows the states and transitions we use in our $3 \mathrm{G}$ model, which are described next. Table 3.1 summarises the model parameters.

States: We define 3 different states in our $3 \mathrm{G}$ model based on the standard states of the RRC state machine: Idle or PCH, FACH and DCH. Since the power consumption for the Idle and $\mathrm{PCH}$ states is similar compared to the power consumed in FACH and $\mathrm{DCH}$, the Idle and $\mathrm{PCH}$ states are grouped together for simplicity and ease of configuration. The power level for each state is fixed, which correspond to the average power at that state. This is a convenient simplification in order to obtain the power values from a power profile.

Next, we describe state demotions, i.e., switching down to less performing states, and state promotions that refer to transitions to a state where the UE experiences higher performance.

State demotions: For each packet $P_{i}$ in the trace and its timestamp $t\left(P_{i}\right)$, we calculate $\Delta_{i}=t\left(P_{i}\right)-t\left(P_{i-1}\right)$ as the elapsed time between the packet and its predecessor. $\Delta_{i}$ is used to simulate progress in the fixed inactivity timers $T_{1}$ and $T_{2}$ : if $\Delta_{i}>T_{1}$ or $\Delta_{i}>T_{2}$, we trigger the corresponding state 
transition. We consider a single inactivity timer value for each of the state demotions (DCH-FACH and FACH-PCH/Idle).

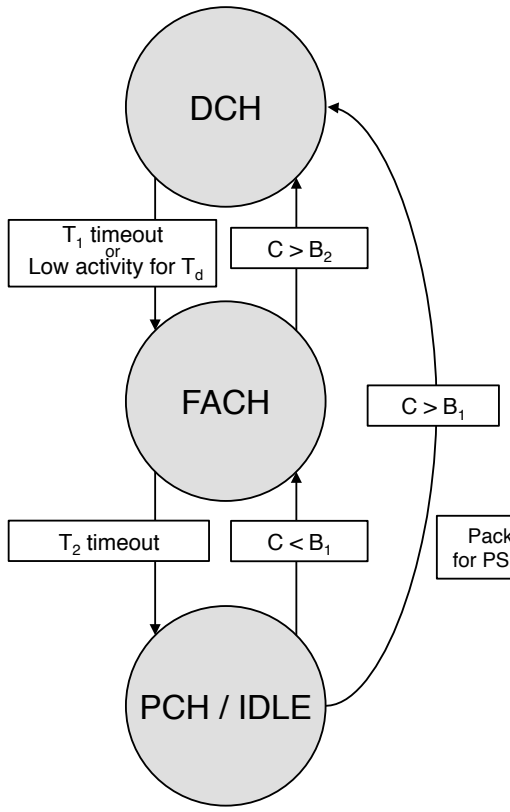

(a)

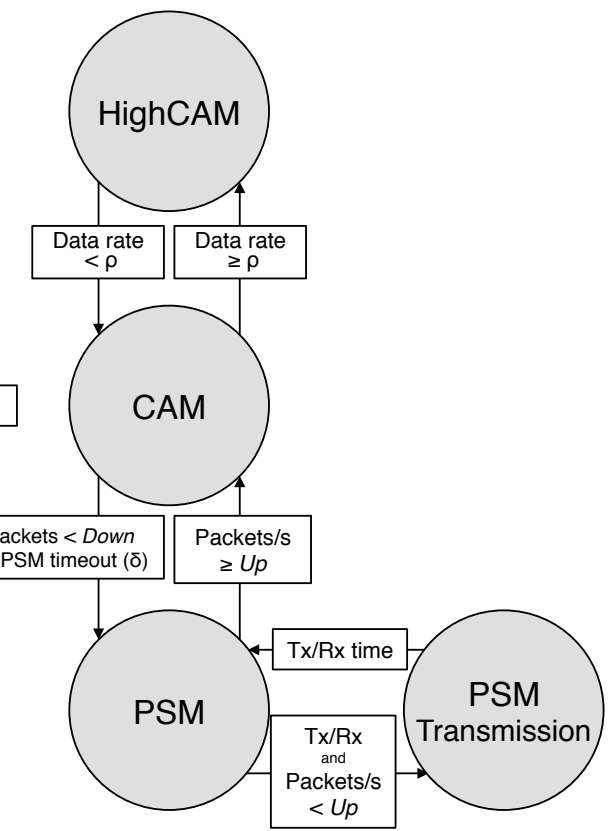

(b)

Figure 3.2: Overview of the state machines for 3G (a) and WiFi (b).

Recall that some networks implement a low activity mechanism in DCH to release the transport channel and switch to FACH before the inactivity timer $T_{1}$ expires [102]. In order to simulate this low activity mechanism, we define $T_{d}$ as the period of time over which the amount of data sent is monitored. We sum the size of packets during $T_{d}$ and force the move to FACH when the data sent is lower than a threshold $D_{d}$. This is the same as using a threshold over the current bit rate that it is used in the standard mechanism.

The fast dormancy mechanism that the UE uses to signal the desire to switch to a low power state before the inactivity timeout is modelled by moving to $\mathrm{PCH} /$ Idle after a predefined time. We represent this behaviour by simply shortening the inactivity timers in case we want to use fast dormancy. For the sake of brevity these transitions are not shown in Fig. 3.2 (a).

Finally, in order to account for the signalling between states, every state demotion has pre-defined a parametrised transition duration which is added to the inactivity timers (e.g., $T_{1}+T_{D C H-F A C H}$ and $T_{2}+T_{F A C H-P C H / \text { Idle }}$ ). We model the energy cost of the transitions assuming that the power during the state demotion is the same as in the FACH state. This approach is adopted to simplify the parameterisation instead of providing an energy 
cost for each transition and each possible signalling protocol.

State promotions: Apart from the standard Idle/PCH-FACH and FACH$\mathrm{DCH}$ promotions, our model considers the direct state promotion to $\mathrm{DCH}$ from a patent [239] as it is shown in Fig. 3.2 (a). Although it is not strictly part of the 3GPP standard, we have chosen to apply this enhancement to cover a major percentage of systems.

The state promotions are controlled by four RLC buffer thresholds: two uplink $\left(B_{1}^{u}\right.$ and $\left.B_{2}^{u}\right)$ and two downlink $\left(B_{1}^{d}\right.$ and $\left.B_{2}^{d}\right)$. When the UE is in Idle/PCH, we use the direction of the packet $P_{i}$ (uplink or downlink) to compare its size to the corresponding threshold $B_{1}^{u}$ and $B_{1}^{d}$. If the size of $P_{i}$ is greater than the corresponding threshold, the UE is moved directly to $\mathrm{DCH}$. Otherwise, the UE is moved to FACH.

The simulation of the state promotion in FACH is performed by simulating the RLC buffer occupancy. We simulate the current occupancy $C$ for each direction $\left(C^{u}\right.$ and $\left.C^{d}\right)$ considering the packets that need to be sent and the data rate of the channel. This is done as follows: since the size and direction (uplink or downlink) for each packet $P_{i}$ is known, $C$ is calculated by adding the size of $P_{i}$ in bytes to the corresponding buffer. Then, when $C>B_{2}$ in any direction, the UE is moved from FACH to DCH.

The data is transmitted (i.e., the buffer is emptied setting $C=0$ ) depending on the channel data rate. First, we define $\Delta_{i}^{u}$ and $\Delta_{i}^{d}$ as the elapsed time from the last uplink or downlink packet (in the same direction) which is calculated from the packet trace. Given $C$ and the channel data rate, the time to empty the RLC buffer $\left(T_{e}\right)$ can be calculated (i.e., when the data is actually sent). Similar to the RLC thresholds, $T_{e}$ also depends on the direction: $T_{e}^{d}$ and $T_{e}^{u}$ for downlink and uplink respectively.

The buffer is emptied when the time to empty the buffer is greater than the inter-packet time in the same direction: $C^{u}=0$ if $T_{e}^{u}>\Delta_{i}^{u}$ for the uplink buffer, and $C^{d}=0$ if $T_{e}^{d}>\Delta_{i}^{d}$ for the downlink buffer. Our model allows to specify $T_{e}$ assuming constant data rate (e.g., $T_{e}=C / 512 \mathrm{kbps}$ ) or as a function of $C$ based on data rate measurements in $\mathrm{FACH}$ for a real network.

Similarly to the state demotions, the transition times are parametrised $\left(T_{I d l e / P C H-F A C H}, T_{I d l e / P C H-D C H}\right.$ and $\left.T_{F A C H-D C H}\right)$. We assume that during that time the power consumed is the same as in the FACH state. For the purpose of estimation this is a simplifying choice, which will potentially result in underestimation in some traces. However, as we will show in section 3.4 the accuracy exhibited is not significantly affected by this choice.

Received signal strength (RSS): The current 3G model does not fully consider the impact of the RSS. The data rate is captured by the recorded input data trace. However, in order to fully model the impact of RSS-power level we would have to feed the EnergyBox with a trace of received signal strength or similar indicator (e.g., Signal-to-Noise Ratio).

This can be added to the implementation if the means of capturing such 


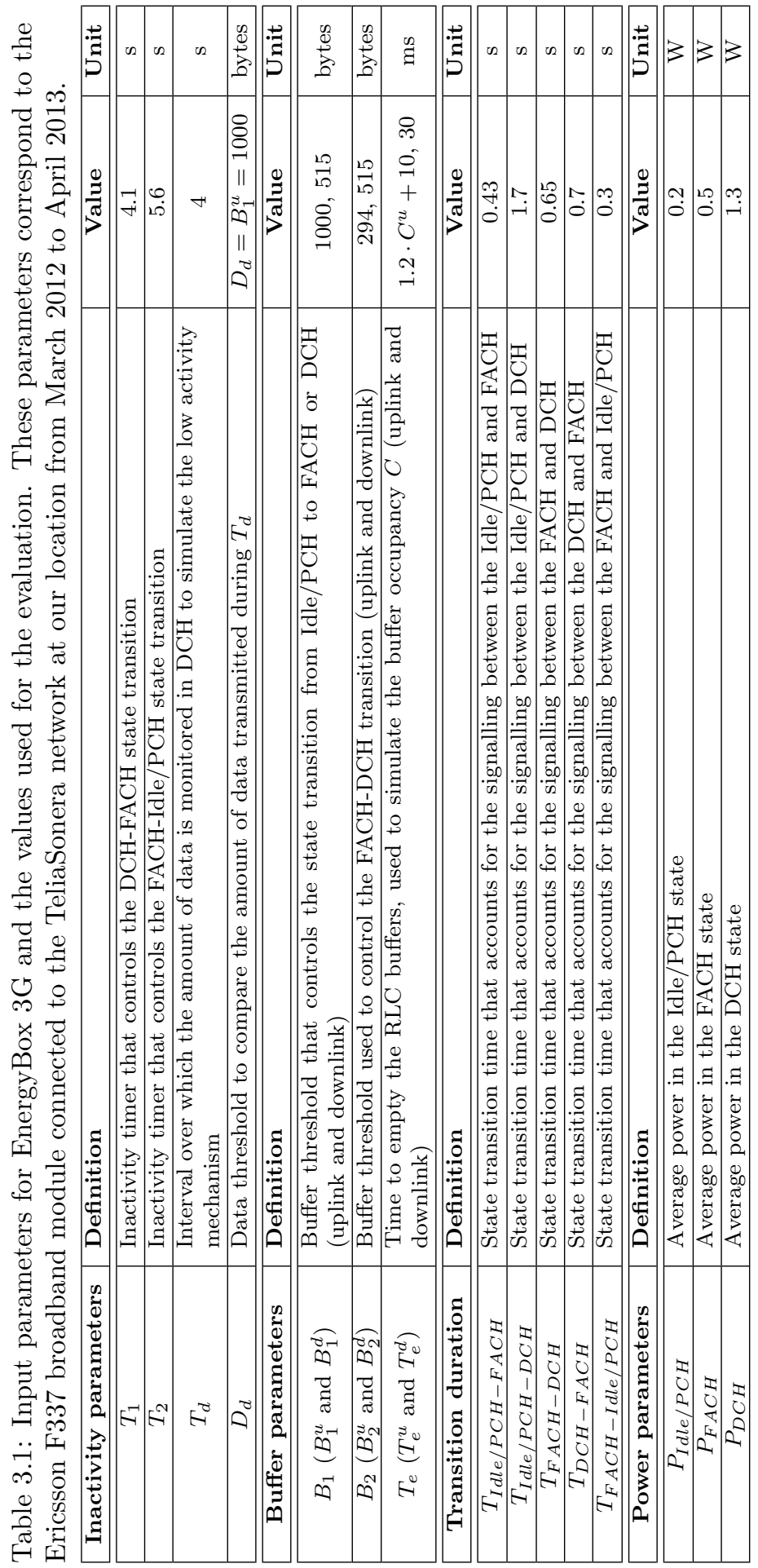


traces are available. In the current state of the model, the fixed power values for the states would have to be manually adjusted in order to calculate a more detailed estimate of energy consumption at different RSS. However, section 3.4 will demonstrate that the accuracy is still at a high level even if the variations in power due to RSS are ignored through using a single constant.

The strength of modelling 3G states in EnergyBox is that it is a generic way of capturing different state machines for different operators using one parametrised finite state machine: if an operator implements a RRC state machine where a single packet triggers a state transition from Idle/ $\mathrm{PCH}$ to $\mathrm{DCH}$, we only have to set the $B_{1}^{u}$ threshold to 0 bytes.

Table 3.2: Input parameters for EnergyBox WiFi and the values used for the evaluation. These parameters correspond to a Samsung Galaxy SII, which does not implement the PSM-TX state.

\begin{tabular}{|c|l|c|c|}
\hline Parameters & Definition & Value & Unit \\
\hline \hline$T_{t}$ & $\begin{array}{l}\text { Timeout time for staying in PSM-TX } \\
\text { after a transmission }\end{array}$ & n.a. & $\mathrm{ms}$ \\
\hline$U p$ & $\begin{array}{l}\text { Threshold that controls the PSM to } \\
\text { CAM transition }\end{array}$ & 1 & packets/s \\
\hline Down & $\begin{array}{l}\text { Threshold that controls the CAM to } \\
\text { PSM transition }\end{array}$ & 1 & packets/s \\
\hline$\delta$ & $\begin{array}{l}\text { Inactivity timer to switch back from } \\
\text { CAM to PSM }\end{array}$ & 200 & $\mathrm{~ms}$ \\
\hline$T_{w}$ & $\begin{array}{l}\text { Time window employed in CAM to } \\
\text { monitor the data rate }\end{array}$ & 50 & $\mathrm{~ms}$ \\
\hline$\rho$ & $\begin{array}{l}\text { Data rate threshold to compare the } \\
\text { amount of data transmitted during } T_{w}\end{array}$ & 3000 & byte \\
\hline \hline Power parameters & Definition & Value & Unit \\
\hline$P_{P S M}$ & Average power in the PSM state & 30 & $\mathrm{~mW}$ \\
\hline$P_{P S M-T X}$ & Average power in the PSM-TX state & n.a. & $\mathrm{mW}$ \\
\hline$P_{C A M}$ & Average power in the CAM state & 250 & $\mathrm{~mW}$ \\
\hline$P_{C A M-H}$ & Average power in the CAM-H state & 500 & $\mathrm{~mW}$ \\
\hline
\end{tabular}

\subsubsection{WiFi model}

The WiFi mode in EnergyBox captures the adaptive PSM mechanisms based on the inactivity timer and the number of packets per second. Stations switch between two states (PSM and CAM) using adaptive PSM, but in order to model the high data rate behaviour of a station, we define the state machine shown in Fig. 3.2 (b). Table 3.2 summarises the parameters of the model.

States: The station only wakes up for beacons in the $P S M$ state (i.e., no data transmission is performed in this state). The PSM-TX state represents the transmission of packets in the PSM mode for those station models that 
switch to a high power only during the transmission interval. The station is awake in $C A M$ when the power-saving features are disabled.

We employ fixed power levels for the PSM, PSM-TX and CAM state, corresponding to the average power drain at each state. This is a convenient simplification in order to obtain the power values from a power profile. However, we have observed that the power drain of the station increases with the throughput as it was illustrated in section 2.2. This behaviour is captured by the $C A M-H$ (CAM high) state.

State transitions: Recall from section 2.2 that some stations implement packet per second thresholds ( $U p$ and Down). In PSM, the station transitions to PSM-TX whenever there is a transmission (Transmit in Fig. 3.2 (right)) and the number of packets per second is lower than $U p$. The transition time between PSM-TX and PSM is defined as a reconfigurable parameter named transmission time $\left(T_{t}\right)$.

In PSM, the transition to CAM is triggered whenever the number of packets per second is higher than the $U p$ threshold. The packets per second is calculated from the packet trace using the inter-packet interval $\Delta_{i}$. When the number of packets per second is less than Down for a predefined timeout time $(\delta)$, the station switches back to PSM. We assume that the duration for this transition is insignificant, and thus we ignore it.

The transition between CAM and CAM-H is modelled as follows: First, the data rate is computed from the packet trace over a short time window $T_{w}$. The data rate is then compared to a threshold $\rho$, and when the threshold is exceeded, the station switches to CAM-H. When the data rate is lower than $\rho$, the station switches back to CAM. We will see later that this twostate approximation of the rate-based behaviour in the (standard) CAM state provides an adequate accuracy in the evaluations.

Similarly to the $3 \mathrm{G}$ model, the current WiFi model does not fully consider the impact of the received signal strength from the power consumption perspective.

\subsection{Evaluating EnergyBox accuracy}

This section describes the evaluation methodology and the settings employed to assess the accuracy of the EnergyBox models against physical energy measurements. Section 3.3.1 explains the methodology and evaluation metrics, section 3.3.2 describes the input packet traces, and section 3.3.3 explains the obtaining of parameters for the EnergyBox models.

\subsubsection{General methodology}

The evaluation of EnergyBox is performed against physical energy measurements. We define two metrics to quantify the accuracy of the EnergyBox: energy accuracy and time accuracy, which will be described leading to equa- 
tions (1) and (2) below. The general methodology is similar for both WiFi and $3 \mathrm{G}$, and follows the following steps for calculating energy accuracy:

1. A set of applications is used to create different traffic patterns. We simultaneously collect packet traces and measure the device power trace $P_{d}(t)$ for transmissions while creating traffic from one application at a time. This is done using the toolset described in Appendix B statically in the same location, where there is little received signal strength variation. The power trace $P_{d}(t)$ represents the ground truth.

2. EnergyBox is fed with the packet traces and outputs the inferred states $S_{i}(t)$.

3. EnergyBox assigns the measured device-specific handset power levels for each state to the inferred states obtaining the inferred power trace $P_{i}(t)$. The energy consumption is computed in joules integrating $P_{i}(t)$ over the trace duration $(T)$. Energy accuracy is measured by the ratio of the inferred energy consumption over the energy consumption of the measured power trace (the ground truth):

$$
\text { Energy Accuracy }=\frac{\int_{0}^{T} P_{i}(t) d t}{\int_{0}^{T} P_{d}(t) d t}
$$

Time accuracy represents the percentage of time that the inferred states and the measured states overlap, i.e., the higher the measure, the greater the overlap, and therefore the better the accuracy over a given time interval. This is a complementary metric used to study the underlying accuracy for estimating the correct state since a perfect energy accuracy does not necessarily imply a high state overlap. Time accuracy is calculated as follows:

1. From $P_{d}(t)$, we compute the expected device states $S_{d}(t)$ using power level thresholds. These thresholds are obtained earlier using measurements for a given device.

2. The states $S_{i}(t)$ inferred by EnergyBox are compared against the expected device states $S_{d}(t)$ and the difference over time is computed. $T$ represents the total duration of a trace and $f(x, y)$ simply returns 1 if the states are the same $(x=y)$, and 0 otherwise. Time accuracy is defined as follows:

$$
\text { Time Accuracy }=\frac{\int_{0}^{T} f\left(S_{i}(t), S_{d}(t)\right) \mathrm{dt}}{T}
$$

\subsubsection{Evaluation packet traces}

The packet traces are 5 minutes long, captured with tcpdump from the WiFi and $3 \mathrm{G}$ interfaces. We demonstrate the reliability and versatility of the EnergyBox by covering a wide range of data patterns in terms of inter-packet 
interval, packet size and total amount of data transmitted created by commonly used mobile applications as described below. Since the EnergyBox is deterministic (i.e., it creates the same output for a given input packet trace and configuration parameters), each packet trace is fed only once into EnergyBox.

We chose to test several different applications with one trace each, similar to the approach in other current work $[64,173,197]$. We employed 10 different packet traces coming from different applications for $3 \mathrm{G}$ and 9 for WiFi, representing a wide range of applications. Email has periodic small data transmissions, whereas Facebook and Web represent bursty downloads of bigger amounts of data. Spotify is a music streaming application that sends data in different bursts and Stream is a constant radio stream. Skype Chat represents instant messaging services which usually have smaller data transmissions. Skype Call and Video are audio and video conferences with some small chat messages. Finally, Youtube captures the user watching videos online.

The packet capture process can result in packet loss in high speed networks due to the high number of packets per second (e.g., 10 Gbps Ethernet) [39]. In our work we assume the captured packet trace to be error free since the packet loss for lower data rates (e.g., $450 \mathrm{Mbps}$ ) is typically low or negligible [221].

\subsubsection{Selection of parameter values}

In order to evaluate EnergyBox we instantiate the parameters of the $3 \mathrm{G}$ and WiFi models as shown in Tables 3.1 and 3.2 respectively. The parameters for $3 \mathrm{G}$ model are based on using the Ericsson F3307 broadband module described in Appendix B connected to the TeliaSonera network at our location. The WiFi parameters are set based on an instance of the Samsung Galaxy SII smartphone model. This section describes the empirical methods employed to collect the parameters for each model in EnergyBox.

Parameters for the $\mathbf{3 G}$ model: The $T_{1}$ and $T_{2}$ inactivity timers of a range of real networks are available in the literature $[24,26,152,177,198]$ or can be obtained from a single power measurement when sending a single packet (from Fig. 2.4 in section 2.2.1) and observing the time spent in each state. Similarly, the transition duration times are obtained from a power measurement while sending data generated by different applications (e.g., the delay observed in Fig. 2.4), or measuring the round-trip time for the different transitions sending various pings in the different states. As a simplification, a single value is adopted for each state transition duration. Each value is set from an average over 10 power measurements.

The RLC buffer thresholds $B_{1}$ and $B_{2}$ from a real network can be acquired experimentally or from the literature. The experimental method used is as follows: for every transition and direction (uplink/downlink), we set the device in the desired state $(\mathrm{PCH}$ or $\mathrm{FACH})$, send a packet with the 
potential triggering size and observe the state transition on live power trace generated by a measurement setup. The size of the packet is increased until the transition takes place resulting in the threshold. Chapter 5 (section 5.2 ) proposes algorithms to automatically estimate the inactivity timers and RLC buffer thresholds.

Once the RLC buffer thresholds are known $\left(B_{2}\right), T_{e}$ (uplink and downlink) can be modelled based on data rate measurements instead of assuming a constant data rate [198]. In order to calculate $T_{e}$, we define a packet of $s$ bytes $\left(s<B_{2}\right)$, a packet of size $b\left(b<B_{2}<s+b\right.$, so that the sum triggers the state transition), and a short delay. We send a packet of size $s$, wait the short delay and send $b$.

If the buffer is emptied when the second packet arrives to the buffer (i.e., packet $s$ has been already sent), no state transition is observed since $s<B_{2}$ and $b<B_{2}$, and thus the short delay is larger than $T_{e}$. Then, in order to estimate $T_{e}$, the short delay is shorten until the state transition occurs. If the buffer is not emptied when the second packet arrives to the buffer (i.e., the data is still not sent), then $s+b$ exceeds the threshold triggering the state transition. The procedure is repeated for different packet sizes of $s$.

Fig. 3.3 shows the resulting $T_{e}^{u}$ in the uplink direction increasing $s$ every 50 bytes ( $\mathrm{x}$ axis). The result is different from assuming a constant data rate. We employ a simple linear regression over the measured values. The $T_{e}^{d}$ parameter is set as a constant to $30 \mathrm{~ms}$ since we observed that the transition to DCH occurred after this delay independently of the packet size.

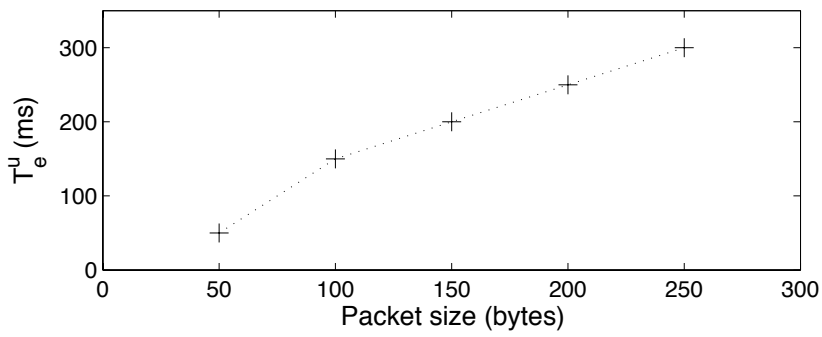

Figure 3.3: Measured time to empty the buffer $T_{e}^{u}$.

The parameters to model the low activity mechanism $\left(T_{d}\right.$ and $\left.D_{d}\right)$ can be experimentally determined. Once the UE is in DCH, it sends data at a constant rate, which is decreased every $a$ seconds $(a=10$ for our measurement) until the DCH to FACH transition occurs. Then, $T_{d}$ and $D_{d}$ can be chosen from the data rate information and power profile.

Finally, the power values are simply obtained from the average power measured for each state. This is measured from the power profile obtained when sending a large packet (e.g., from Fig. 2.4 in section 2.2.1). We obtained the values in our lab environment where the received signal strength did not vary significantly. 
Parameters for the WiFi model: The parameter $U p$ is obtained by increasing the number of packets sent per second and observing the transition to CAM in the power trace. Similar methodology can be used for Down once the station is in the CAM state. The parameter $\delta$ is directly obtained from the power trace or observing the time interval between the NULL frames that the device sends to the access point to describe the state change. These values are also available in the literature for different devices [140,192].

We set $U p=1$ and Down =1, which are the settings of the stations used in our measurements (Samsung Galaxy SII and the Sony Ericsson Xperia Arc). The Samsung Galaxy SII was used for the evaluation in section 3.4 and $\delta$ was set to $220 \mathrm{~ms}$ based on observation of the power trace.

The $T_{t}$ parameter can be obtained by sending few packets of different sizes in the PSM state and observing the power profile (similar to Fig. 2.7 in section 2.2.2). However, the device used for the evaluation moves to CAM for every packet transmission, and therefore we do not instantiate this parameter or use the PSM-TX state.

The power levels are obtained from the measured power profile when sending few packets. We select 30 and $250 \mathrm{~mW}$ for PSM and CAM respectively, based on measured average power levels at the same location.

The selection of the parameters and the power level to model the CAM-H are set as follows: First the $T_{w}$ needs to be set. A short window is suggested (e.g., $50 \mathrm{~ms}$ ) to adapt to the rapidly changing data rate of WiFi stations. Then, the $\rho$ threshold and the power value for CAM-H can be decided by sending a sequence of packets with different inter-packet intervals (similar to Fig. 2.8) and measuring the power drain. The power drain for CAM-H should be chosen as the average of high end of the power profile observed. The selection of the power level for the CAM-H state becomes a compromise: a too high power value would lead to an overestimation of consumed energy, whereas a too low value can underestimate it. $\rho$ is set to the point when the power drain significantly increases (i.e., power at time $5 \mathrm{~ms}$ in Fig. 2.8).

While estimation of power values for a device may not be representative for that device family generically, we trust that the selection of the values in Tables 3.1 and 3.2 does not invalidate the accuracy results of the tool in section 3.4: (1) the device measured parameters are within the range of similar measurements performed on devices from similar generation of devices by other researchers $[64,120,173,213]$, and (2) several devices from different manufacturers (LG, Samsung and Sony) were subjected to our tests and we believe that any potential production dependent variations could not have influenced the accuracy of the tool experiments in section 3.4 significantly.

\subsection{Evaluation results}

The results from experimenting with the application traces detailed in section 3.3.2 are described first for the $3 \mathrm{G}$ model, followed by the WiFi model. 


\subsubsection{Accuracy of EnergyBox 3G}

Fig. 3.4 shows the measured energy consumption for the different traces (i.e., the ground truth) and the energy estimated by EnergyBox. The average energy accuracy of EnergyBox over the different traces is $98 \%$.

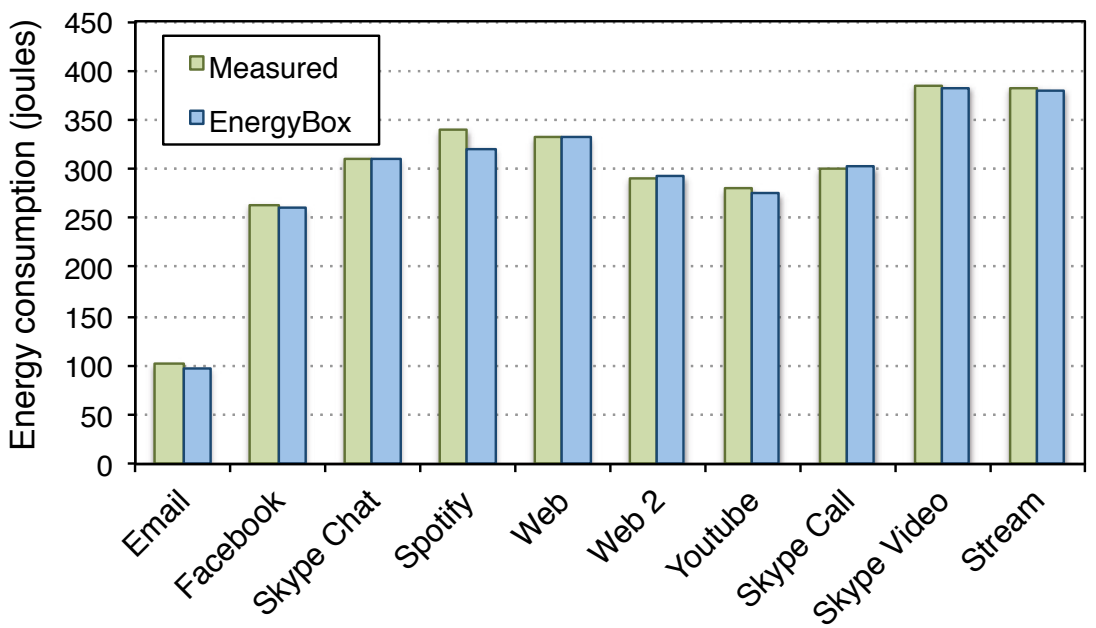

Figure 3.4: Energy consumption of the measured trace and EnergyBox 3G estimation for different traces.
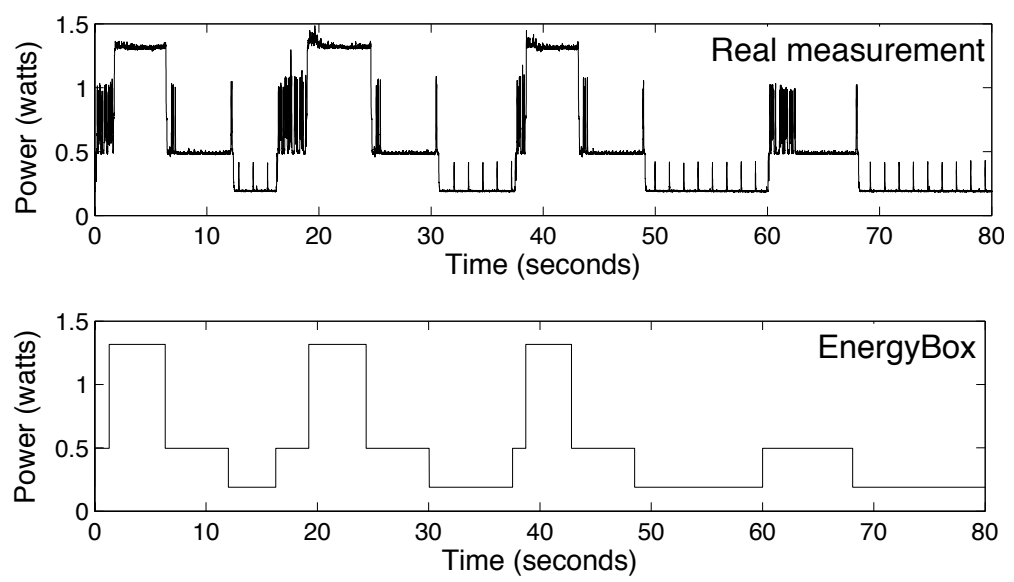

Figure 3.5: A fragment of a 3G measurement and EnergyBox 3G inferred output for the Email trace.

The lowest registered energy accuracy is for the Email and Spotify traces. Fig. 3.5 shows an example of the EnergyBox accuracy by comparing it to the real measurements for the Email trace. The source of inaccuracies in 


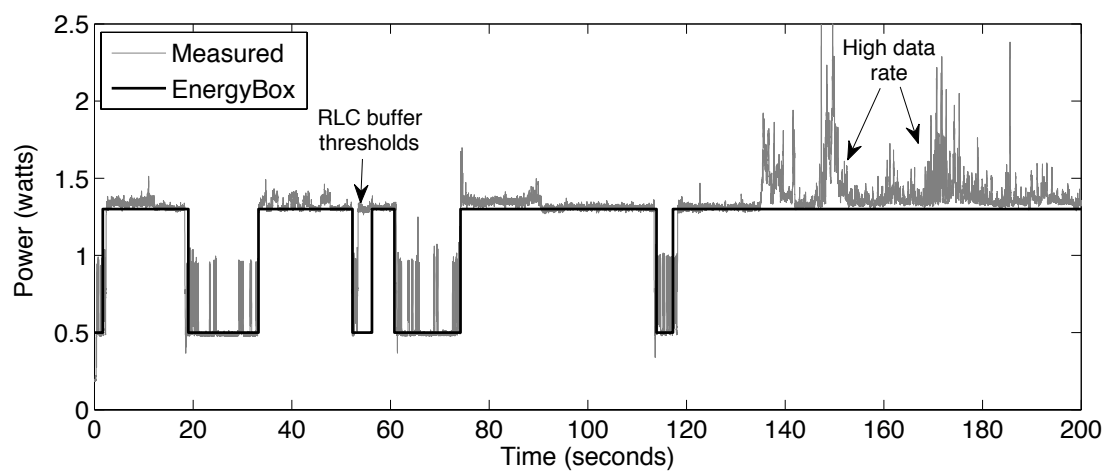

Figure 3.6: A fragment of a $3 \mathrm{G}$ measurement and EnergyBox 3G inferred output for the Spotify trace.
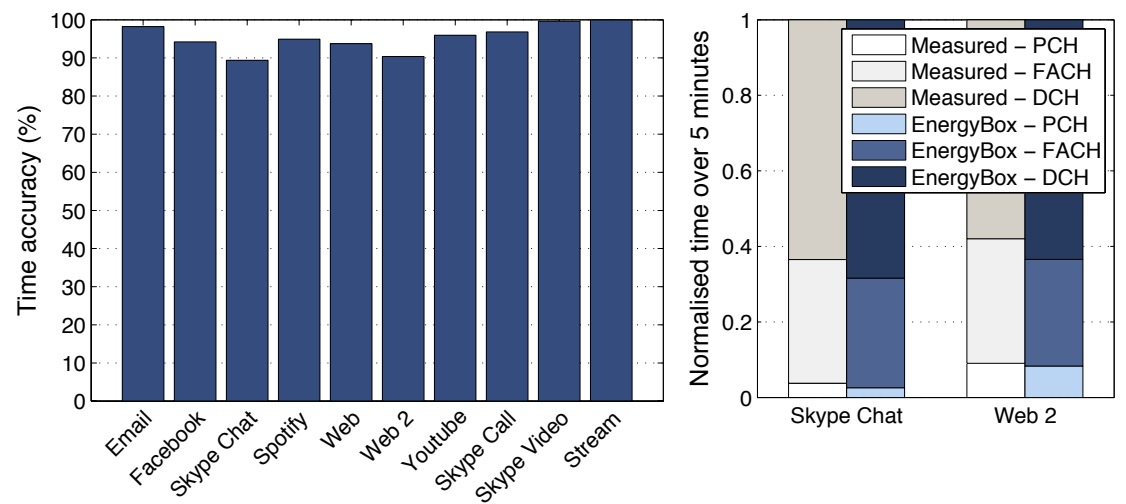

Figure 3.7: 3G time accuracy for all the traces (left) and time spent by the UE in each state for Skype Chat and Web 2 (right).

the Email trace is assuming that the signalling energy cost is the same as in the FACH state.

The source of inaccuracies for the Spotify trace are shown in Fig. 3.6. EnergyBox employs a constant power level for DCH thus not considering the slightly higher power for higher data rates. The RLC buffer simulation errors as pointed out in Fig. 3.6 are discussed below when reviewing time accuracy. Nevertheless, the energy accuracy for both Email and Spotify traces is still quite high (96\% and $94 \%$ respectively).

Fig. 3.7 (left) shows the time accuracy in accordance with equation (2) in section 3.3 .1 for all the traces. The average time accuracy is $95 \%$, meaning that the deduced state by the EnergyBox coincides with the actual state in the device in $95 \%$ of the experiment interval. Regarding lower time accuracies (e.g., $89 \%$ for Skype Chat or $90 \%$ for Web 2), the typical cause is that the inferred state is DCH while the real state is FACH. The source 
of this deviation is the time to empty the buffers $\left(T_{e}\right)$ of the RLC buffer simulation. Even though the modelling of this parameter is based on real measurements, the variations in number of active users and traffic at the operator network makes the real time to empty the buffers different from the measurement-based model in some instances. The Skype Chat trace has many small packets. These create many switches in the RLC data buffer simulation. However, the accuracy is still close to $90 \%$.

\subsubsection{Accuracy of EnergyBox WiFi}

The average energy accuracy of EnergyBox over the different traces for WiFi is $98 \%$. Fig. 3.8 shows the measured energy consumption for the different traces (the ground truth) and the estimated energy consumption.

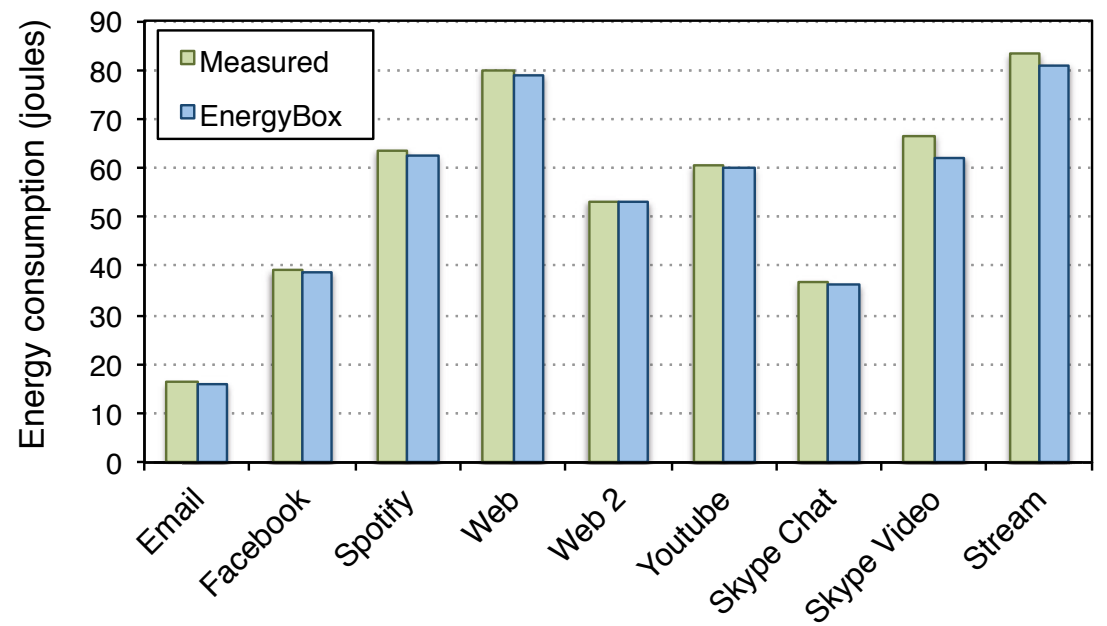

Figure 3.8: Energy consumption of the measured trace and EnergyBox WiFi estimation for different traces.

The lowest accuracy is for Skype Video (93\%). The differences can be traced to traces with higher data rates (e.g., Skype Video and Stream). Our two-state abstraction of rising power for higher data rates with the fixed power level for CAM-H does not fully cover the actual power drain with the chosen fixed value associated with CAM-H. However, the discrepancy is still relatively small.

Detailed study of the contribution of the CAM-H abstraction reveals that this state contributes with $4 \%$ increase in accuracy on average over all the traces. As expected, the contribution is higher in high data rate traces such as Youtube (10\% increase) compared to traces with low data rate like Email (0.03\% increase).

Fig. 3.9 (left) shows the time accuracy for all the traces (note that y axis starts at $80 \%$ ). Since the adaptive PSM in the Samsung Galaxy SII dif- 

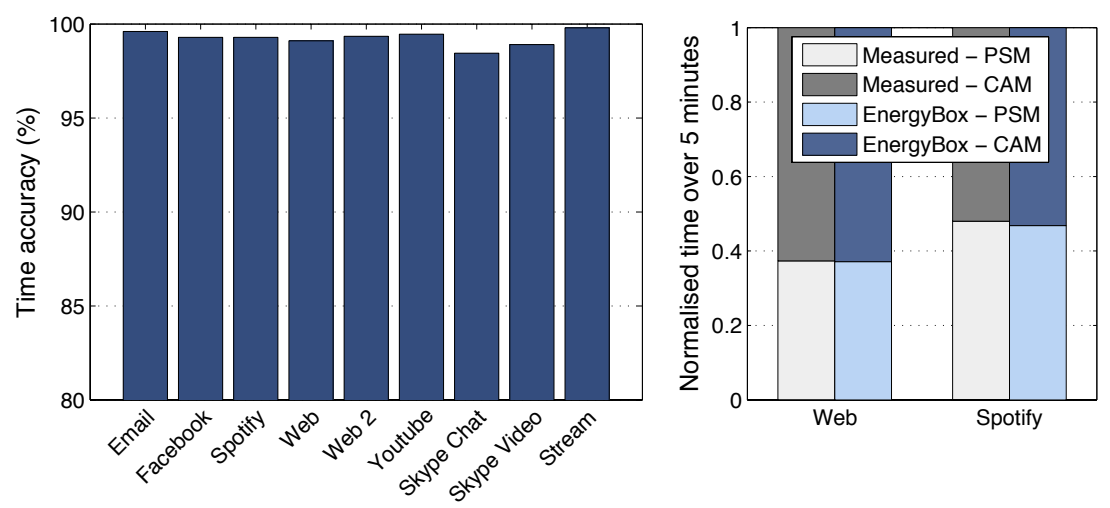

Figure 3.9: WiFi time accuracy for all the traces (left) and time spent by the station in each state for Web and Spotify (right).

ferentiates only two states (PSM and CAM), we perform the time accuracy evaluation of the EnergyBox WiFi using the two basic states of our WiFi state machine. The average time accuracy is $99 \%$. The few discrepancies are originated from the value given to $\delta$, which makes the switch from CAM to PSM somewhat earlier than in the real states.

Fig. 3.9 (right) shows the time spent in each state for the traces with the lowest time accuracy. The discrepancies are very small.

To summarise, the above comparisons provide evidence that EnergyBox is an accurate tool for estimating energy and device state information for a wide range of real traces.

\subsection{Discussion and extensions}

Designing energy-efficient solutions for wireless networks starts by energy awareness. To reduce energy waste, there is a need to provide visibility and understanding of the energy consumption that impacts battery lifetime at the user end as well as elsewhere in the network. Our contribution makes the energy footprint of the network interface explicit by providing a tool to quantify its energy consumption. The tool enables efficient, flexible and accurate energy consumption studies given packet traces as input.

EnergyBox targets the currently most widespread wireless interfaces $(3 \mathrm{G}$ and $\mathrm{WiFi}$ ) and captures the parameters influencing the energy consumption most. We build on the fact that the handset energy consumption in $3 \mathrm{G}$ is essentially driven by the inactivity timers and the RLC data buffer thresholds that control RRC state transitions at the network operator end. The energy consumption in WiFi is mostly influenced by the handset dependent adaptive PSM mechanism implemented at driver level in the handset. We capture these aspects within parametrised state machines in the EnergyBox 
and evaluate its accuracy against physical measurements. Our evaluation shows that the EnergyBox provides accurate energy consumption estimates.

EnergyBox has been extended in different directions which are summarised in the following subsections.

\subsubsection{Open source project}

We provide our code ${ }^{1}$ to the research and development community offering a simple to configure but still powerful approach to perform accurate data communication energy measurement studies.

The EnergyBox project started from a EnergyBox version developed by the author. The current version was developed as part of the bachelor thesis of Rihards Polis [185] supervised by the author to include a convenient graphical user interface in addition to the already available command line. It has been further extended by the author and Jens Green Olander, who refactored the code base and improved the performance as part of his master thesis [87] (also supervised by the author).

\subsubsection{Modelling extensions for 3G}

The evaluation section shows that EnergyBox has two modelling limitations. The fixed power level for the different states is a convenient simplification which also leads to inaccuracies in high data rate cases. In addition, including the impact of the received signal strength was left as an extension.

A part of the bachelor thesis by Rasmus Holm [101] models the impact of the data rate and the received signal strength on the power consumption for $3 \mathrm{G}$ using the data collected by the author. Systematic experiments are performed while collecting power measurements in the Ericsson $3 \mathrm{G}$ module at different locations at university.

Every $10 \mathrm{dBm}$ (from $-50 \mathrm{dBm}$ to $-110 \mathrm{dBm}$ ) the following tests are performed: (1) file upload and download, (2) small packet transmission and a big packet transmission and (3) file upload and download at different fixed data rates. The modelling results in adding dynamic power levels to the most consuming states based on RSS and data rate. While it can improve the accuracy, the power model is device specific.

Including the impact of the received signal strength into EnergyBox enables to study the impact of dynamically changing location while transmitting the data. However, this incurs some complexity for the user of the tool at the time of designing the scenario. The user would need to provide an received signal strength trace as well as the packet trace as input.

\footnotetext{
${ }^{1}$ https://github.com/rtslab/EnergyBox/
} 


\subsubsection{Long Term Evolution (4G) model}

EnergyBox was extended with a Long Term Evolution (LTE) model based on the RRC state machine for LTE [29]. The work is done as part of the bachelor thesis of Gunnar Berg supervised by the author and follows a similar methodology to the one used to develop the 3G model. The thesis models the standard LTE RRC state machine and instantiates the model for a particular network (Tele2 $4 \mathrm{G}$ ). The results show an energy accuracy of 90-98\% for different real traces compared to physical power measurements in a mobile device (Samsung Galaxy S5). While this work is at a preliminary stage it shows that the tool set has a potential for extension towards future generation of technologies.

\subsubsection{Cellular interface emulation over WiFi}

While the current version of EnergyBox provides the energy consumption values for collected traces, the users need to capture the packet traces using a $3 \mathrm{G}$ network which can incur extra cost. In addition, the feasibility of testing a mobile application under diverse and realistic network settings is limited by the testing environment, where different conditions are generally hard to mimic. The delays introduced by different cellular operator settings, variations of signal strength or network handovers are a few examples of relevant network conditions that the application tests can consider.

An interesting extension to EnergyBox is to employ the network characterisation included in the network models to emulate different cellular interfaces. This is proposed by the author and performed as part of the master thesis of Alexander Alesand at Spotify [8]. The work implements a system where the data traffic of a mobile device connected via a WiFi connection is shaped to mimic a $3 \mathrm{G}$ connection. The high correlation between the resulting packet traces and authentic $3 \mathrm{G}$ traces shows the viability of the approach. The system also provides test repeatability since the cellular parameters are under control of the testing environment.

\subsubsection{Automated testing environment at Spotify}

EnergyBox has been conveniently adopted as part of the automated testing suite at Spotify for the continuous integration pipeline [87]. The work by Jens Green Olander analyses the traffic patterns of the Android client of Spotify and significantly reduces its $3 \mathrm{G}$ energy consumption in measured scenarios by $22 \%$ to $54 \%$.

In the following chapters we will focus on the uses of EnergyBox for 3G energy estimation in order to reduce the consumption. 
"Thank you Mario! But our princess is in another castle!"

Toad

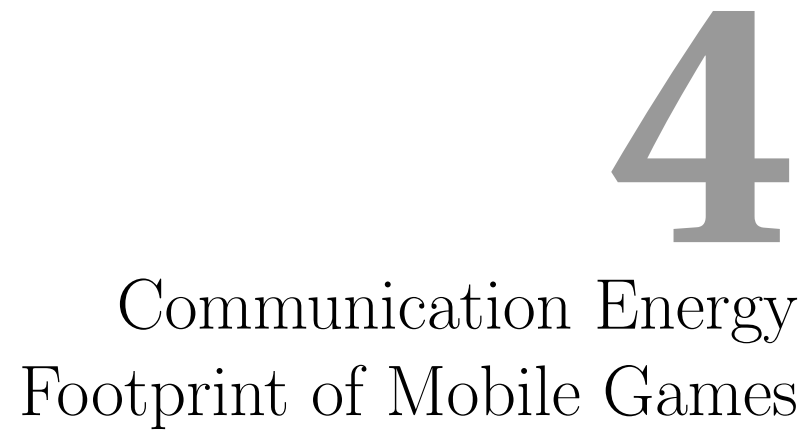

As we exemplify in this chapter, the communication energy landscape of mobile applications is still underdeveloped. When it comes to energy as a resource, the mobile device efficiency is dependent on device hardware, a complex interaction between the platform services (e.g., Android services), communication provider (telecom operator), and the thousands of apps that a user may download on the device. Quantifying the communication energy footprint of applications is undeniably essential to develop means to reduce the energy consumption.

Although a significant proportion of the mobile applications are games ${ }^{1}$, there has been little attention paid to their specific characteristics with respect to communication energy. Thus, in this chapter we focus on the communication energy footprint of a selection of free Android mobile games, and characterise their comparative energy use employing EnergyBox. The chapter illustrates the usage of EnergyBox to reveal inefficient data patterns and provide guidelines for improvement. Each mobile game is analysed alone by quantifying its energy consumption in isolation $E(\{i\})$. The experimental tests reported in this chapter were performed by Mathias Almquist and Viktor Almquist as a Bachelor thesis [9] under the supervision of the author.

In particular we select 20 mobile games amongst the top 100 Android official market free games and create a substantial data set consisting of

\footnotetext{
${ }^{1}$ Google Play Store offers 1.5 million applications where around $18 \%$ of all applications are games. Source: http://www.appbrain.com/stats/android-market-app-categories, accessed 26 February 2015.
} 
actual data communication traces while playing the games over a total of 25 hours. To the best of our knowledge this work is the first attempt at creating a large enough dataset of free mobile games played by human players for investigating the data patterns and cellular communication energy consumption properties. This dataset is available to the research community for further analysis.

The analysis of the energy for communication over a $3 \mathrm{G}$ network indicates that there is a wide variation among the games, the largest footprint being 8 times higher than the lowest one. The results also indicate both app-specific and game category-specific relations between data pattern and energy use. Finally, we show that background traffic not relevant to the gameplay can be a major source energy waste.

The structure of the chapter is the following. Section 4.1 describes the game selection methodology laying out the interesting criteria for the comparative study. Section 4.2 describes our evaluation setup and metrics, followed by section 4.3 in which individual and comparative analysis of the 20 games with respect to communication energy is carried out. Finally, section 4.4 concludes the chapter by summarising the results and providing suggestions for improvement.

\subsection{Mobile game dataset selection}

A core aspect of our study is the selection of a set of games to be profiled, which need to be representative in terms of popularity, type and characteristics. The selection of games needs to provide diversity to support a study with a wide range of communication patterns and their impact on energy consumption. We consider the following game properties:

- Gameplay: While it is natural to select games with different gameplay to provide diversity, we also select games with similar gameplay in order to compare them. We consider simple and advanced games in terms of graphics that can impact the CPU usage.

- Multiplayer game: Online multiplayer games are expected to generate more traffic than single player games since they communicate with other players and/or servers.

- Input type: We categorise the input type of mobile games based on a previous analysis [141]. The state of a game with a time-insensitive input changes only when an input is performed by the user (e.g., TicTac-Toe). A time-sensitive input is required in games where the state can change without user interaction, e.g., driving games or controlling a character's movement.

- Advertisements: We limit this work to free games and therefore we also study whether the games contain advertisements with an impact on the amount of data sent and energy consumption. 
- Release date: Selecting current games as well as older games allows us to observe potential improvements in terms of energy consumption.

The games are selected from the top 100 free games from the Google Play Store on 26th May 2014 based on (1) the above described properties, and (2) a subjective coverage of the known game types based on authors' gaming experience. Selecting games based on the above properties provides us the possibility of comparing aspects such as sequels and predecessors, single player and online multiplayer games, current and earlier games or games with different gameplay.

Tables 4.1, 4.2 and 4.3 show the selected games categorised as single player time-sensitive, single player time-insensitive and multiplayer online games.

\subsection{Evaluation setup and metrics}

Since our aim is to quantify the energy consumption due to realistic playing sessions, for our evaluation a human player interacts with the game under test. The games are installed as a common user would proceed, i.e., by downloading them from the application market to a mobile device (Samsung I9300 Galaxy S3 running Android 4.1.2). Each game is considered a black box since the source code is not available.

- Trace collection: We collect the packet traces while playing the game under test using tcpdump. Only the traffic of the game under test is allowed using a iptables-based firewall to block the rest of the traffic. The packet traces are later used to compute the energy consumption using EnergyBox.

- Number of tests: Each of the 20 games is played 5 times for 15 minutes. Since the gaming sessions are quite repetitive, playing longer sessions does not necessarily add more interesting information. We play 75 minutes per game and 25 hours in total.

- EnergyBox parameters: The parameters of EnergyBox correspond to the operator TeliaSonera measured in our local experimental area described in section 3.3.3. The device-specific UE power values for the RRC states are based on earlier measurements for a mobile device [198]: $612 \mathrm{~mW}$ and $416 \mathrm{~mW}$ for the power consumption in $\mathrm{DCH}$ and FACH respectively. We set the power in $\mathrm{PCH}$ to $0 \mathrm{~W}$ in order to quantify only the energy spent for data communication since the measurement period is the same for all games.

- Evaluation metrics: The metrics used to analyse the collected data are the amount of data sent and total energy consumption. These are interesting from the user perspective since they directly impact user's battery lifetime and data plan quota. 


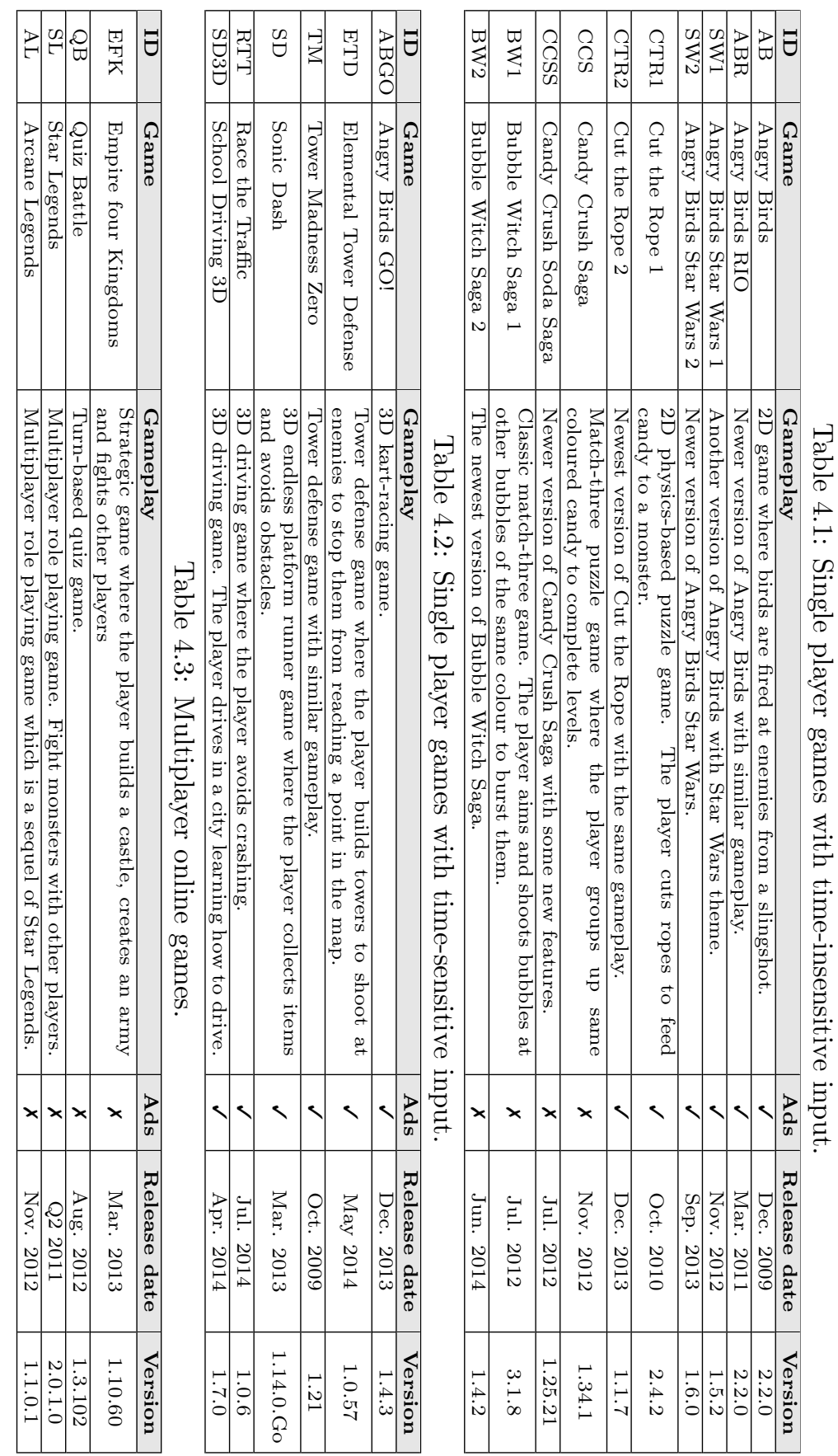


We also analyse the time spent by the UE in the different RRC states as well as the number and type of RRC state transitions that provide valuable insight regarding the impact of the data pattern and the RRC state machine. The number of RRC state transitions is a representative metric correlating with the amount of signalling created between the UE and the network operator. For example, a single FACH-DCH state transition is associated with 10 message signalling transmissions [199], which introduces additional delay and energy consumption at the user end and an overhead for the network operator end.

\subsection{Communication energy footprint of games}

This section presents the results regarding $3 \mathrm{G}$ communication energy, amount of data sent and RRC state machine statistics.

\subsubsection{Energy consumption and data traffic}

Fig. 4.1 shows the average energy consumption due to communication as well as the standard deviation of the 5 repetitions per game. The games are grouped by the categories as presented in Tables 4.1, 4.2 and 4.3.

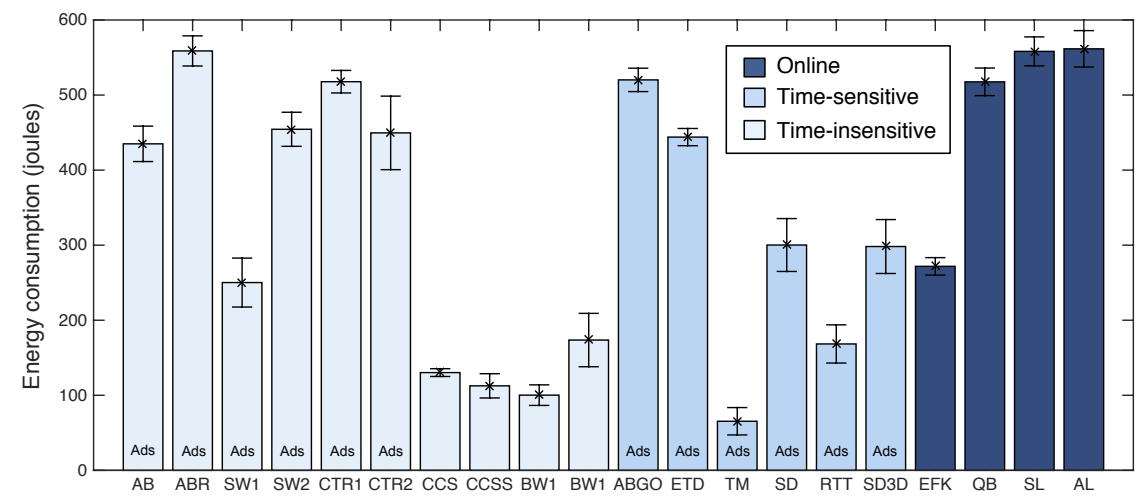

Figure 4.1: Average energy consumption and standard deviation for the selected games resulted from playing each game 5 times for 15 minutes.

There is an evident diversity in energy consumption among the 20 studied games. The most and least consuming games are Arcane Legends (AL) and Tower Madness (TM) consuming 561 and 65 joules respectively. The results show that half of the games present a high energy consumption (more than 400 joules). Games with similar gameplay and characteristics such as the Angry Bird games (AB, ABR, SW1 and SW2) present very different energy footprint. 
Multiplayer online games (AL, SL and QB) are among the highest consumers as expected. Surprisingly, single player games like Angry Birds (AB, ABR, SW2 and ABGO), Cut the Rope (CTR1 and CTR2) and Elemental Tower Defense (ETD) consume as much as online multiplayer games.

Next we analyse the amount of data exchanged by the games and its relationship with energy consumption. Fig. 4.2 shows the average data traffic and standard deviation for the 20 games and Fig. 4.3 shows the energy and data traffic relationship in a scatter plot.

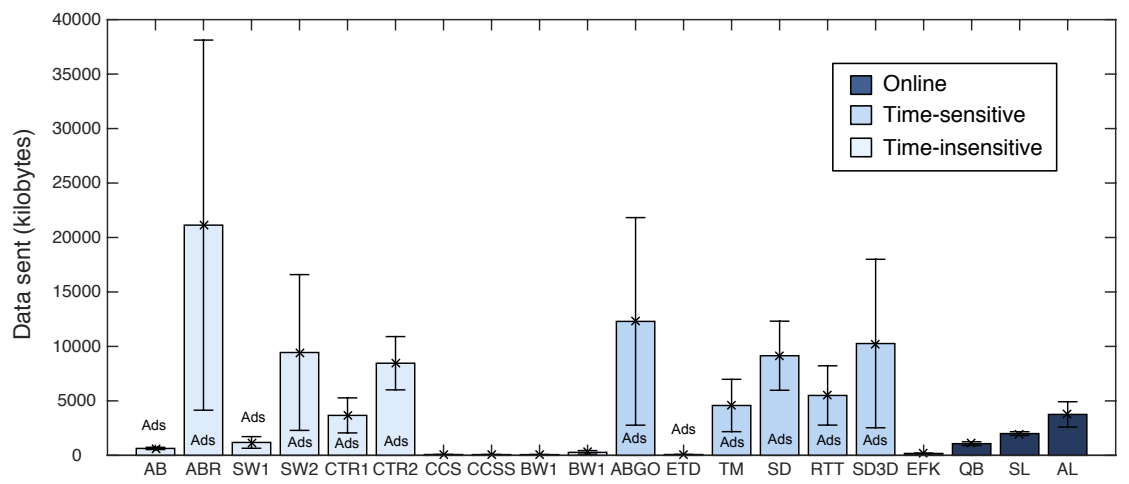

Figure 4.2: Average data exchanged and standard deviation for the 20 games.

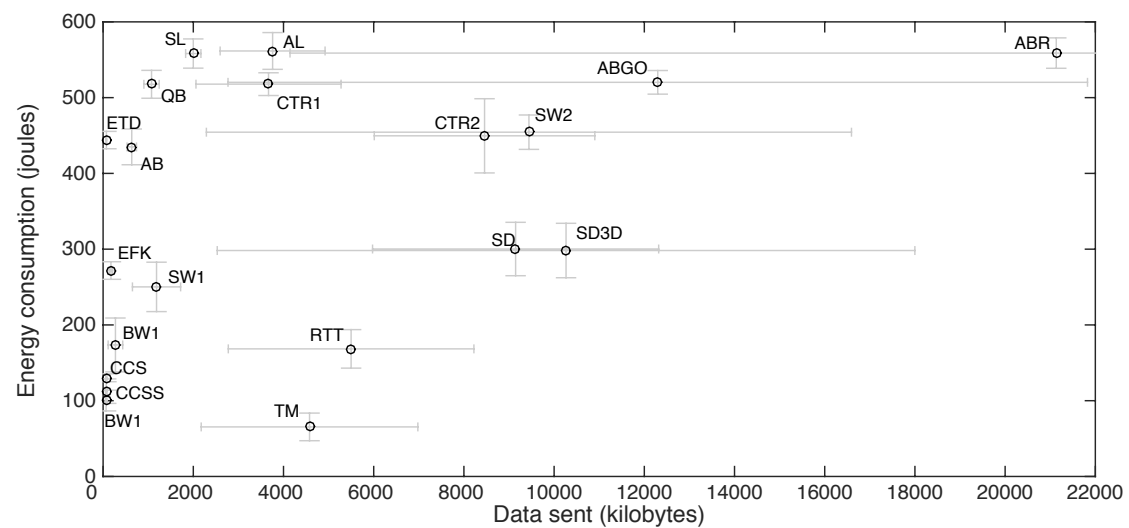

Figure 4.3: Scatter plot showing the average energy consumption and data sent for the 20 games.

Similarly to energy consumption, Fig. 4.2 shows that there is a big diversity in the amount of data traffic. We also observe a large standard deviation in Fig. 4.2 for the games with the largest amount of data sent, 
such as Angry Birds RIO (ABR). The number of ads fetched and statistics sent is different for each game and the ads come in different formats (e.g., video or images), which results in a large deviation.

The difference in amount of data sent by the single player games with and without in-game advertisements is enormous. Only 4 out of the 16 single player games are ad-free (CSS, CCSS, BW1 and BW2) and are developed by the same company. These ad-free games present low energy consumption as well as low amount of data sent. These games serve as an illustrative example of the consumption of games without any ads. It is interesting to point out that the lowest consuming game (TM) also contains ads.

It is noticeable from Fig. 4.3 that the energy consumption is not proportional to the data sent. There are multiple games showing that the energy consumption is high while the amount of data exchanged is low. For example, Elemental Tower Defense (ETD) consumes 7 times more energy than Tower Madness (TM) while TM sends approximately 63 times more data than ETD. We will return to analyse the reason of this in more detail in the next section.

Surprisingly, we show that all games except the multiplayer online games can be played without connectivity. 16 out of 20 games can still be played if we enable firewall to block the network traffic of the games. It makes the high energy consumption unjustifiable from the user perspective and points out the need to reduce their energy consumption.

\subsubsection{UE states and data pattern}

Clearly, a game should make the UE spend the largest percentage of time in the $\mathrm{PCH}$ state to reduce its consumption and avoid staying long in $\mathrm{FACH}$ and DCH. Fig. 4.4 shows that the RRC state distribution differs greatly which indicates different data patterns. We show the RRC state distribution for the trace with the median energy for each game.

The most consuming games (ABR, SL and AL) spend approximately 95\% of the time in DCH. Games with similar gameplay such as Angry Birds (SW1, SW2 and ABR) or Sonic Dash, School Driving 3D and Angry Birds GO (SD, SD3D and ABGO) present very different RRC state distributions, showing that there is room to improve the networking behaviour of these games and their energy footprint.

The multiplayer game Empire Four Kingdoms (EFK) spends a significant percentage of the time in $\mathrm{PCH}$ and $\mathrm{FACH}$ reducing the time in $\mathrm{DCH}$. Even though the game sends packets often, these are small enough to not exceed the RLC buffer threshold and avoid expensive transitions to DCH. Empire Four Kingdoms (EFK) consumes less than the other online multiplayer games (QB, SL and AL).

A more detailed analysis of the individual packet traces reveals that most transmissions are used for fetching ads, sending game statistics such as achievements, score or items purchased, and encrypted data that we cannot 


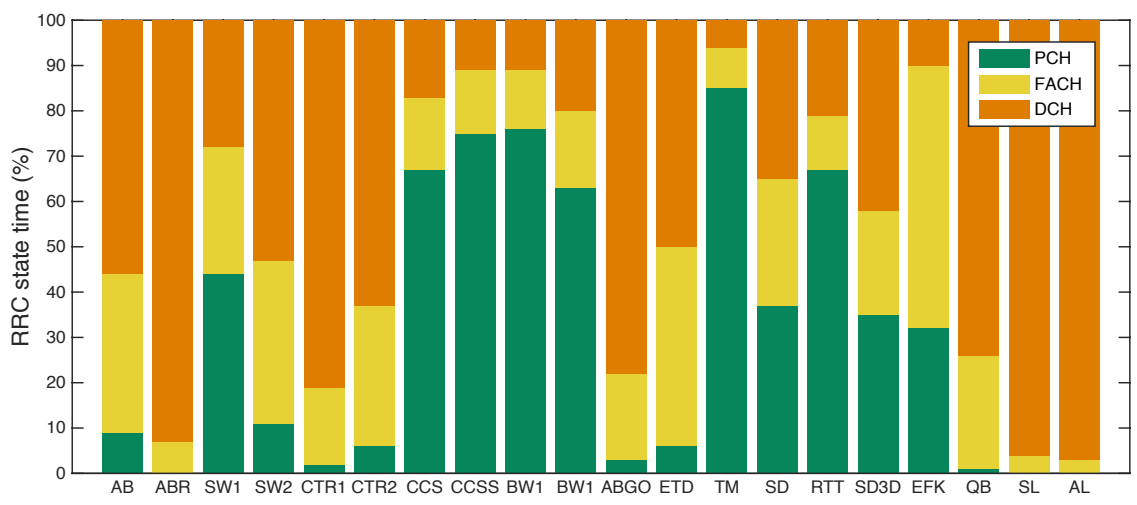

Figure 4.4: Distribution of time spent by the UE in the different RRC states for each game.

access. For example, the single player games without ads (CSS, CCSS, BW1 and BW2) communicate with the company's servers (King) as well as cloud computing service providers (Amazon). The game statistics sent by Bubble Witch 2 are accessible in plain text.

We look in more detail at Tower Madness (TM), since it is the lowest consuming game even though it employes in-game advertisements, and compare it against ETD. We observe that ETD transmits data constantly and fetches ads frequently (roughly every $20 \mathrm{~s}$ ) spending most of the time in FACH and DCH. Instead, TM fetches ads only when a new level is entered (roughly 5-10 mins) staying most of the time in PCH. However, as previously described, TM sends much more data since it receives video ads in large files while ETD receives small images. TM is a good example of how to keep the energy consumption low while still using ads.

An interesting observation from Fig. 4.4 is that out of the single player games with in-game ads, the majority of games with time-sensitive input (TM, SD, RTT and SD3D) spend more time in PCH than the ones with time-insensitive input (AB, ABR, SW2, CTR1 and CTR2). The majority of games fetch ads when entering, restarting or completing levels. We observe that completing a level in the selected time-insensitive games takes short time (approximately $40 \mathrm{~s}$ ) resulting in more frequent downloads than for time-sensitive games. Between 2 to 10 mins are needed to complete a level in the latter. Thus, the time to complete a level seems to impact the energy consumption through the ad-fetching frequency.

Fig. 4.5 shows the number of state transitions performed by the UE when running each game. We show the run with the median energy consumption. There is a noticeable difference among the multiplayer games, where Star Legends (SL) and Arcane Legends (AL) are real-time games requiring frequent updates compared to the other games (QB and EFK). Some 


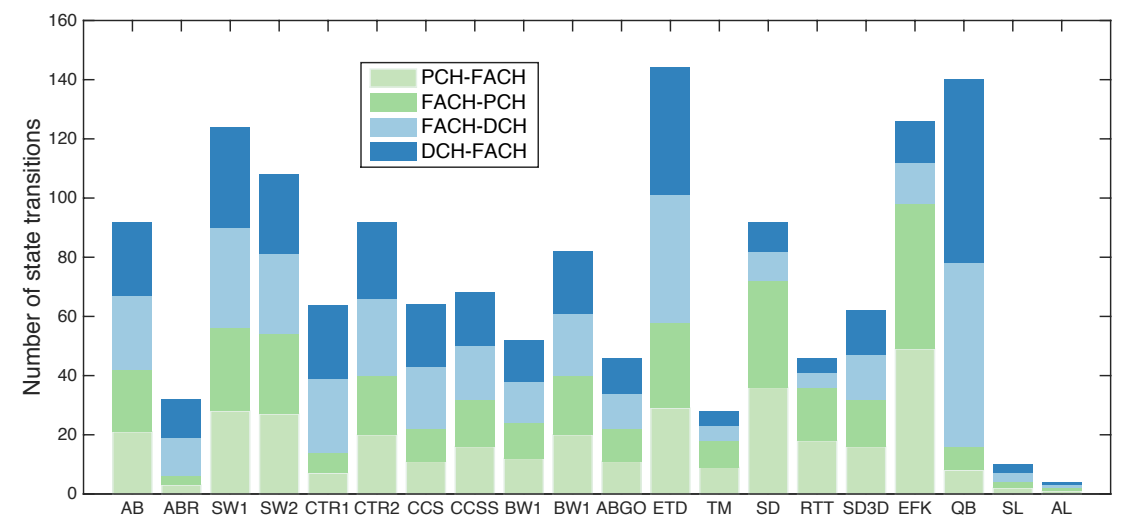

Figure 4.5: Number of RRC state transitions for the 20 games.

games (SD, RTT and EFK) employ small data transmissions resulting in a majority of PCH-FACH transitions. This appears as a favourable behaviour which avoids transitions to expensive DCH state allowing them to send data at lower energy cost.

To sum up, our results show that there is room for improvement and that the energy consumption can be reduced if we focus on communication.

\subsection{Beyond energy awareness}

Energy efficiency starts from energy awareness, and quantifying the energy consumption is an essential first step to reveal inefficiencies. Thus we strongly recommend developers to integrate the freely available energy simulation tools such as EnergyBox within their testing environment. These tools can help to reveal inefficiencies in a similar way to our study.

As part of our initial findings using EnergyBox, the dataset confirms the fact that games (like other mobile apps) do not have an energy-proportional communication over $3 \mathrm{G}$. The analysis revealed a wide variation in consumption among the selected top free games, but also confirmed the expectation that ad-free games have much lower data traffic and lower energy consumption in general. However, there is evidence that it is feasible to combine low energy consumption and advertisements by employing efficient data patterns. Also, it is currently possible to play the games by disabling network connections during playing to avoid the additional communication energy.

Next we lay out a number of suggestions to lower the energy consumption for mobile games. These general suggestions are related to techniques which are further analysed in the subsequent chapters of the thesis.

Extending the idle time of the wireless interface is a clear goal in order to reduce energy consumption. Once the source of inefficiency has been identified by performing studies like the one in this chapter, one can devise 
methods to improve energy efficiency. For example, even though the amount of data sent is not proportional to energy consumption, reducing the number of transmissions over time is a simple starting point, which can easily extend the idle time of the interface.

Developers can identify the required minimum functionality of the application and which data exchanges contribute to the intended goal of the application, or ultimately to the user experience. For example, game content like statistics can be categorised as background data if this content is not a priority but it is still desired. Then, the traffic can be deferred for a more energy-efficient operation. In this respect, the next Chapter 5 proposes a traffic aggregation algorithm to coordinate the background traffic originated from different applications and send it in an energy-efficient manner.

The study showed that downloading ads inefficiently can be very expensive and that the majority of the games download ads periodically or when entering, restarting or completing a level. While showing ads is a foreground task which cannot be deferred, prefetching can be employed to download several ads at once to decrease the fetching frequency. This can reduce the energy consumption of games with short level completion times or short ad update periods. For video ads, downloading them at once at the highest data rate is recommended, since the user often needs to watch the whole video before starting the game.

These app-specific improvements require tailor-made solutions, such as selecting the most efficient ad content provider or setting an appropriate ad or game statistic sending frequency based on level duration. In Chapters 6 and 7 of this thesis different tailor-made solutions are studied for different contexts: Chapter 6 studies design alternatives (different protocol and data format alternatives) for location-sharing applications, and Chapter 7 studies the cost of a commonly employed mechanism and its settings for instant messaging applications.

To sum up, this chapter has shown that energy awareness is the starting point for further energy optimisations and that EnergyBox is a valuable tool in this regard. The next chapters focus on techniques to improve the energy efficiency from different perspectives. 
"If a thing's worth doing, it's

worth doing late."

Frederick Oliver

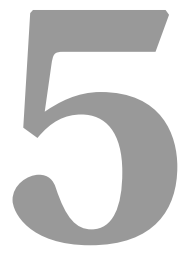

\section{Energy-Aware \\ Cross-Layer Burst-Buffering}

This chapter proposes an online background traffic scheduling algorithm that trades QoS (in terms of delay) for energy consumption. The solution aims to reduce the energy consumption of a particular traffic class originated from a set of applications $E(S)$. It combines multiple transmissions in a single energy event and adapts the communication pattern. Additionally, the chapter shows that by being aware of the energy consumption characteristics of the lower layers of the system (cross-layer information) we can increase the energy savings compared to an uninformed approach. We use EnergyBox to perform the evaluation of the energy savings.

Most applications are unaware of the communication energy characteristics of cellular networks, where the energy consumption is not proportional to the amount of data sent. In particular, the background traffic created by applications (e.g., periodic transfers or updates) leads to high energy consumption, even though its contribution to the total traffic is not high.

As an illustrative example of the energy consumption impact of the background traffic on the battery depletion, 10 minutes of active background traffic (no user interaction or use of the device and the screen-off) can consume $1.6 \%$ of the energy of a fully charged average battery $(2000 \mathrm{mAh}$ at 3.7 $\mathrm{V})$ without the user noticing it. According to data traffic collected from 20 volunteers [107], 35\% of the total traffic was categorised as screen off traffic which consumed $58 \%$ of the total radio energy consumption.

Applications generate traffic with different requirements (e.g., delay sensitive traffic or best-effort), and thus approaches to potentially reduce the 
energy consumption need to consider these requirements. We argue that developers can categorise the different transmissions of their applications based on criteria such as application requirements or essential features, and down-prioritise less important but still necessary communication in terms of QoS. Then, the traffic classes originated from different applications can efficiently be adapted (e.g., by the operating system) to create energy-efficient traffic and reduce $E(S)$.

In this chapter we focus on developing a generic solution that reduces the energy consumption of the elastic and best-effort background traffic created by applications that does not have any strict QoS requirements. Background traffic is often categorised within the background traffic class specified by the QoS of the third generation UMTS standard. The UMTS background traffic class is characterised by low bandwidth requirement, intermittent and asymmetric (uplink/downlink) data transmissions and a permissible delay since the destination does not expect the data within a certain time. The lack of strict timing or performance requirements creates an opportunity to explore the performance-energy tradeoff.

The chapter is organised as follows. We first motivate the need of knowledge about the radio layer parameters to perform energy-efficient background traffic transmissions in cellular networks in section 5.1. Since these parameters are not available for application layer, we propose algorithms to infer them in section 5.2. The algorithm is described in section 5.3 and evaluated in section 5.4 .

\subsection{Motivation}

Our approach is motivated in the fact that the impact of network parameters such as inactivity timers, RLC data buffers thresholds or quality of radio links on the energy consumption of the UE is high. Figure 5.1 shows an illustrative example where small data packets from a data trace are aggregated and sent in a burst causing a state transition to $\mathrm{DCH}$, and higher energy consumption than sending them in FACH. In the original uplink data traffic 7 packets of 100 bytes are sent every 300 milliseconds. When these packets are aggregated to send in burst they lead to $42 \%$ higher energy consumption. In order to schedule the background traffic in an energy-efficient manner, the knowledge of the radio layer parameters becomes essential.

Previous algorithms $[24,137,212,277]$ perform data aggregation and burst transmissions because they are not aware of radio layer parameters such as RLC data buffer thresholds. Obviously this is not optimal in the sense that these approaches will aggregate small data transfers causing state transitions to the most consuming state when unnecessary.

Since network parameters are not available for the application layers, we developed algorithms to provide estimates of the state transition timers and buffer thresholds to our scheduling algorithm. 

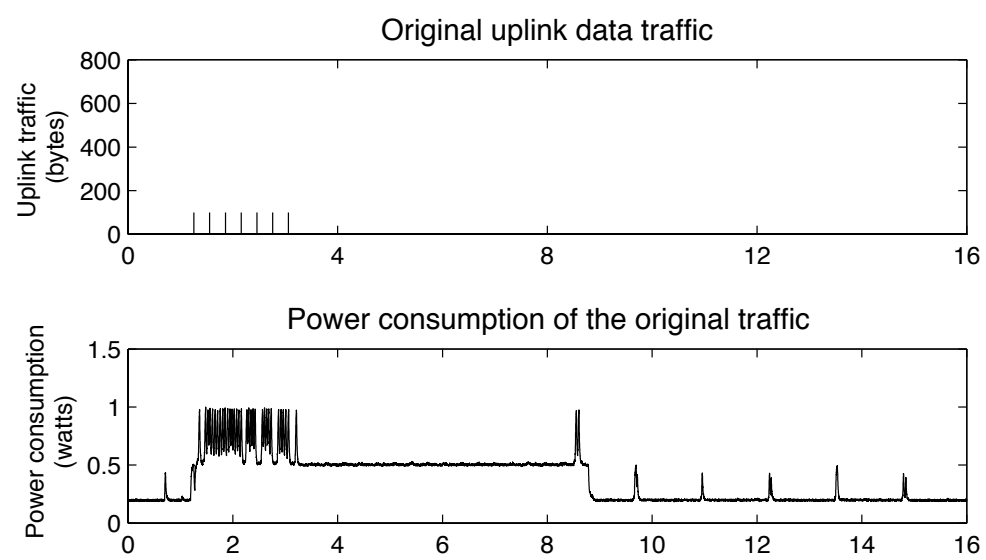

Data aggregation of small packets
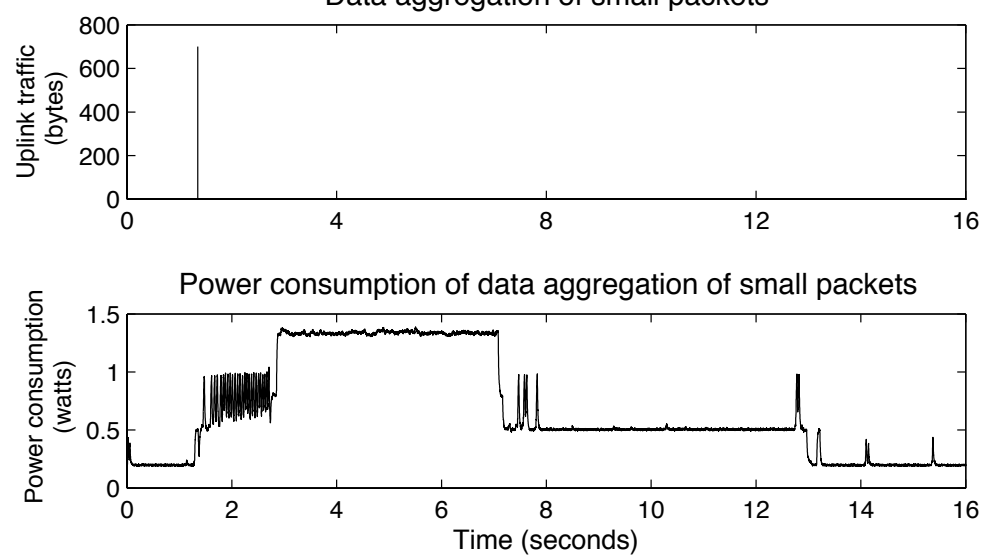

Figure 5.1: The effect of small packet aggregation on energy consumption.

\subsection{Parameter inference algorithms}

In this section the algorithms to infer the inactivity timers and the RLC data buffer thresholds are presented. These are later on used by the background traffic shaping algorithm. While these algorithms are focused on 3G networks, a similar methodology can be employed for other types of networks. 


\subsubsection{Inactivity timer estimation algorithms}

The algorithms to infer $T_{1}$ and $T_{2}$ (inactivity timers from the RRC state machine) are based on the knowledge that each state has qualitatively different round-trip time (RTT) for a data packet. The estimation of the inactivity timers can take place whenever a change of the network parameters is suspected. We call this a ReconfigurationEvent. To calculate RTT, we send synthetic test packets with predetermined sizes from the UE. A server echoes the answer allowing the computation of RTT. We adopt two different approaches for estimating $T_{1}$ and $T_{2}$. In both estimations the UE starts in the $\mathrm{PCH}$ state. Before presenting the algorithm in detail (Algorithm 1), we provide an overview below.

Overview: For the DCH timer $T_{1}$, we trigger a state transition to $\mathrm{DCH}$ by sending a large test packet followed by a sequence of small packets. At some point, the UE will switch down to FACH state due to the small packet sizes. At this time, the longer RTT will be the indicator of the state transition. Using this process we also have an indication of the RTT in the DCH and FACH states (DCH-RTT and FACH-RTT).

For the FACH timer $T_{2}$, the above regime would not work. Sending small packets would keep the UE in FACH indefinitely. Therefore, we begin by a guess for lower and upper bound of $T_{2}$. Note that the upper and lower bound need not be accurate values. We can start by any guess and iteratively converge to $T_{2}$ by reducing the gap between them. We start with a trial timer value between the bounds. We send a test packet that triggers $\mathrm{FACH}$, wait for the trial timer and adjust the lower and upper bounds based on the UE state. If UE is in $\mathrm{PCH}$, the chosen trial timer is longer than $T_{2}$. If $\mathrm{UE}$ is in FACH, the trial timer is shorter than $T_{2}$. Using binary search and a sequence of send-wait-checks we converge to an accurate value of $T_{2}$. Algorithm 1 describes the two estimation procedures.

Input variables: InterPacketInterval and TestPeriod are used for inferring the value of $T_{1}$. InterPacketInterval defines the time between the sending of test packets and TestPeriod is the duration of estimating the value of $T_{1}$. Note that this period has to be longer than the actual (unknown) $T_{1}$. As described above, LowerBound and UpperBound are used for the binary search of the value of $T_{2}$ until the gap between them is smaller than MinGap. Note that UpperBound should be longer than the actual (unknown) $T_{2}$. All values are specified in seconds.

Algorithm description: We further describe the part of Algorithm 1 that infers $T_{1}$ (lines 2-15) using an example. We define as large test packet a packet that will trigger the $\mathrm{PCH}-\mathrm{DCH}$ state transition (e.g., 1200 bytes). A small test packet is the minimum packet size. Figure 5.2 shows the RTT values computed by running Lines 3 -12. In line 13, the DetectRTTJump procedure simply identifies a RTT increase in orders of magnitude. In the example, the increase shown for packet number 9 indicates a DCH-FACH 


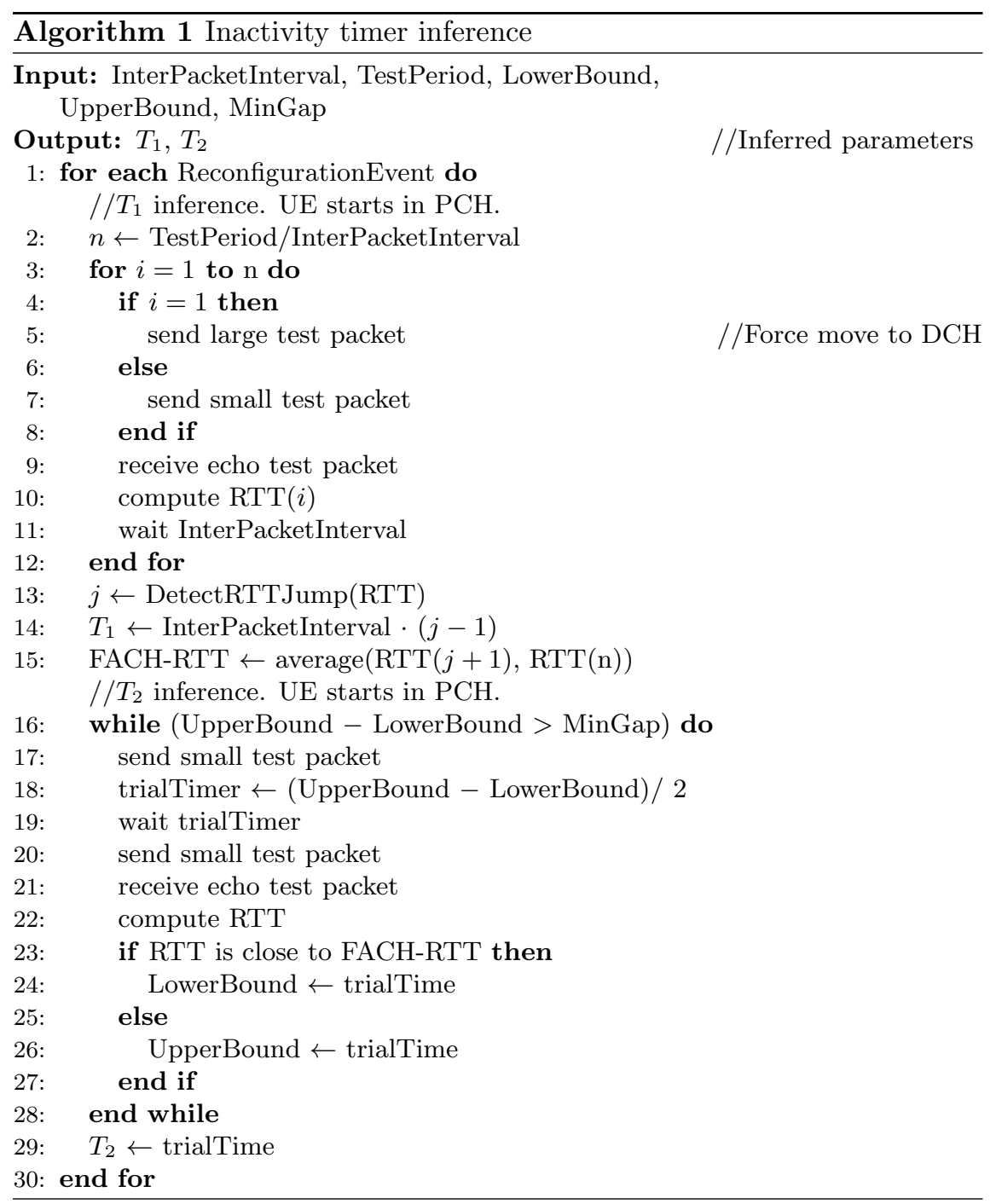


transition. Therefore, the procedure returns $j$ equal to 9 , which leads to the value of $T_{1}$ to be 4 seconds ( 8 times 0.5 ).

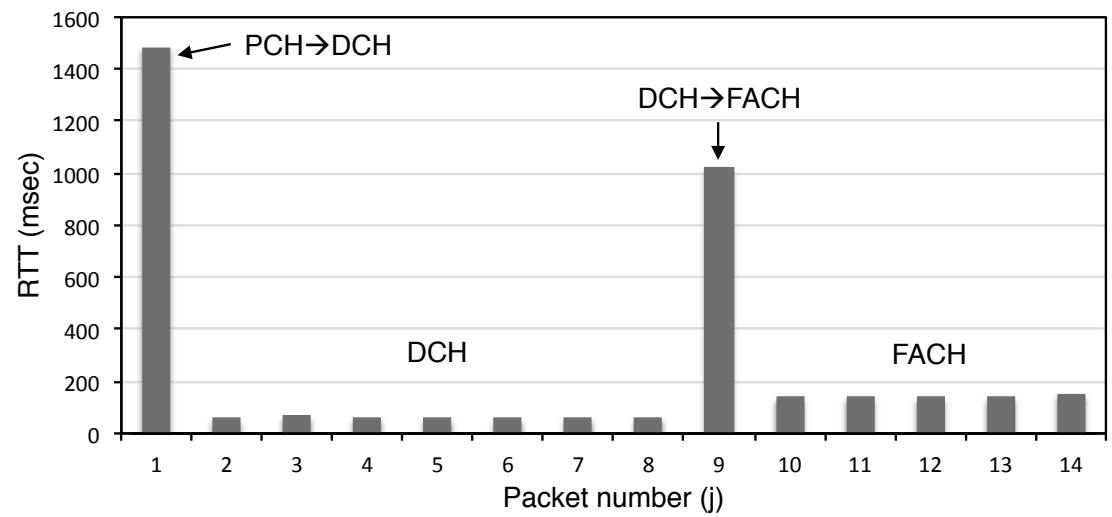

Figure 5.2: RTT values obtained for $T_{1}$ inference. In the example InterPacketInterval is set to 0.5 seconds and TestPeriod of $T_{1}$ is 7 seconds.

Lines 16-29 infer the inactivity timer $T_{2}$ by performing the aforementioned binary search. In line 17 the UE sends a small packet that triggers the transition to FACH state. The UE waits trialTimer seconds and checks its state by computing the RTT. If the state is still FACH, $T_{2}$ is longer than the trial time and the lower bound is updated. Otherwise, if the state of the UE is PCH, $T_{2}$ is shorter than the trial time and the upper bound is updated. This process continues until the gap between the lower and upper bound is smaller than MinGap.

\subsubsection{RLC data buffer threshold estimation}

We next describe the algorithm for inferring the RLC data buffer thresholds that trigger state transitions. There are typically four thresholds, two for the PCH-DCH transition $\left(B_{1}^{u}\right.$ and $\left.B_{1}^{d}\right)$ and two for FACH-DCH transition $\left(B_{2}^{u}\right.$ and $\left.B_{2}^{d}\right)$ for uplink and downlink. Algorithm 2 describes our approach for inferring the $\mathrm{PCH}-\mathrm{DCH}$ and $\mathrm{FACH}-\mathrm{DCH}$ thresholds respectively. As in the previous algorithm, we use the RTT to infer the state of the UE by sending a small test packet and receiving the echo from the server.

For inferring the PCH-DCH data buffer threshold, we use an estimate of the buffer size denoted by an upper and lower bound (MaxBytes and MinBytes) in an analogous manner to Algorithm 1. $T_{1}$ and $T_{2}$, inferred by the previous algorithm, are used to define a boundary for every trial starting from PCH. This is shown in lines 1-17 of Algorithm 2.

For inferring the FACH-DCH data buffer threshold, we adopt a different approach. In this case, by triggering fewer state transitions we save energy used up for inference. This part of the algorithm works in two phases. In phase one, we approach the buffer threshold using large increments to the 


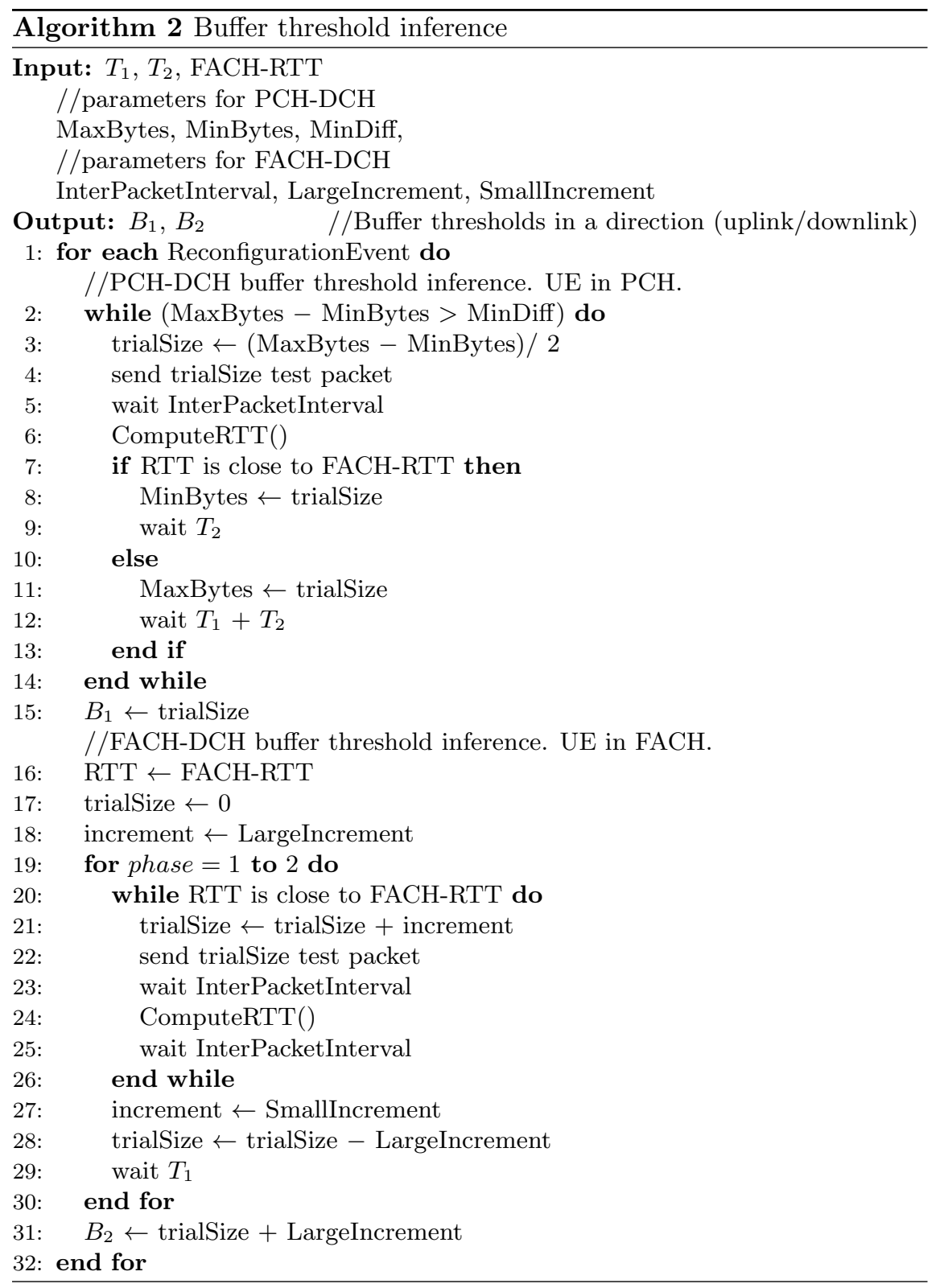


test packet size. In the second phase we use small increments in order to fine-tune the estimate of the threshold.

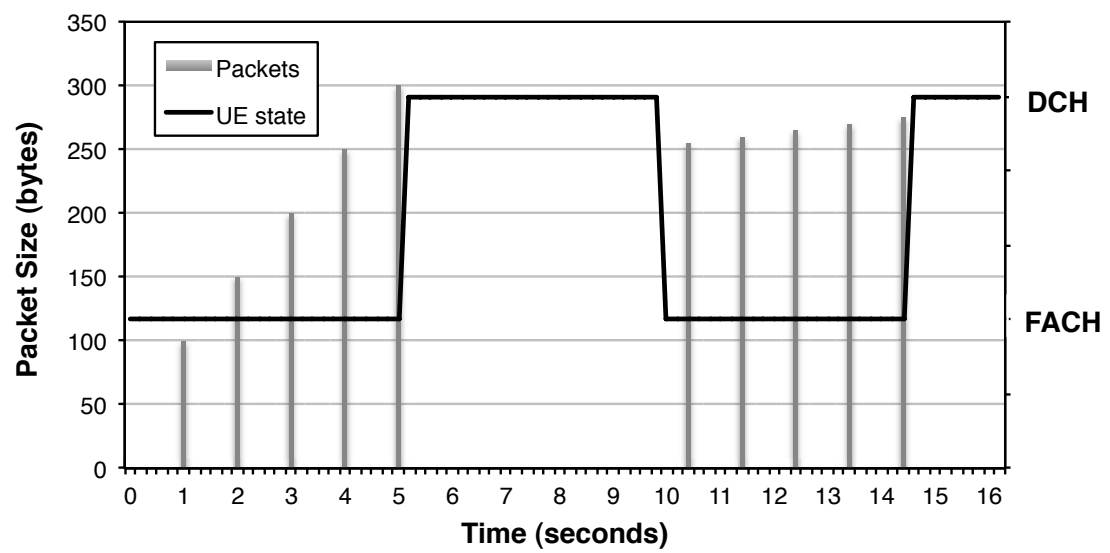

Figure 5.3: Illustration of lines 18-34 of Algorithm 2 showing the packets sent, their size and the state of the UE.

We illustrate the second part of the part of Algorithm 2 that infers the FACH-DCH buffer threshold in Fig. 5.3. The impulses show the time and the size of the sent trial test packets (the packets to compute the RTT are omitted). The test packet size is increased in FACH by a large increment (50 bytes) until the DCH state is triggered. The 300 bytes sent in time point 5 triggers the state transition, therefore the buffer threshold is between 250 and 300 bytes. The RTT calculated with each packet is used to infer the current UE state. Then, after waiting $T_{1}$ to return to $\mathrm{FACH}$ from $\mathrm{DCH}$ in time point 10, the size increment is set to SmallIncrement and the algorithm starts sending 250 bytes plus the increment bytes again (e.g., 260 bytes in time point 10). Note that the InterPacketInterval has to be large enough to allow the clearing of the RLC data buffer. The example in Fig. 5.3 uses an InterPacketInterval of 1 second.

\subsubsection{Evaluation of inference algorithms}

In this section we experimentally validate the algorithms to infer the inactivity timers and the buffer thresholds, and evaluate their energy cost and elapsed time to complete. We are interested in the energy cost in order to later understand whether the cost of inferring the parameters drastically reduces the energy savings or not.

We implemented Algorithms 1 and 2 in Java using UDP packets to calculate the RTT. A simple UDP server was developed to echo UDP packets. The algorithm to infer the buffer thresholds (Algorithm 2) was also implemented in the UDP server to infer the downlink thresholds. The energy 

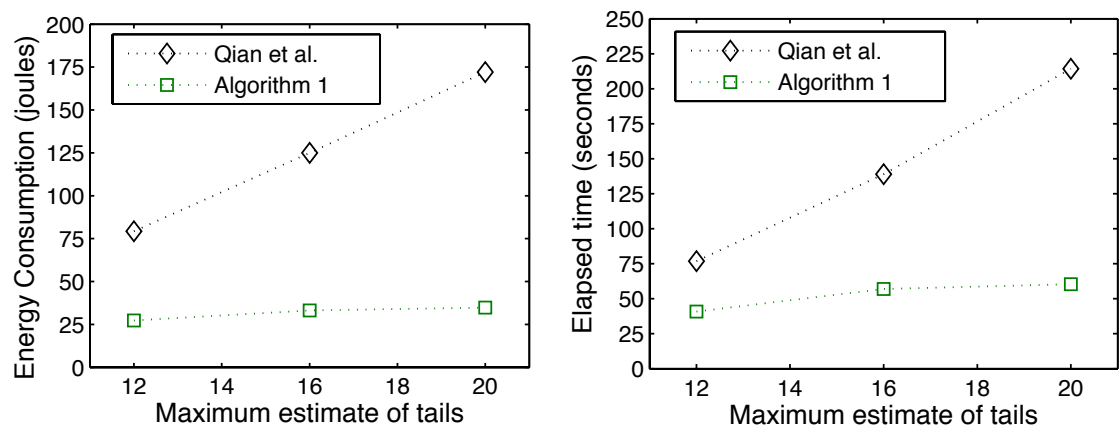

Figure 5.4: Comparison of algorithms in terms of energy consumption and elapsed time.

consumption of the algorithm was evaluated using the broadband module described earlier.

Algorithm 1: We compared the values inferred by the algorithm to the time that the UE stays in $\mathrm{DCH}$ and $\mathrm{FACH}$ (observed in the recorded power trace) when triggering a $\mathrm{PCH}-\mathrm{DCH}$ transition. The settings used in the implementation of Algorithm 1 were the following: small and large tests packets were set to 43 and 1200 bytes respectively ( 1 byte UDP packet and an arbitrarily large one). InterPacketInterval was 0.5 seconds, providing the $T_{1}$ with a granularity of 0.5 seconds. InitialGuess, LowerBound, UpperBound and MinGap were set to 7, 0,16 and 0.5 seconds for the tests respectively. The UpperBound needs to be larger than the sum of $T_{1}$ and $T_{2}$, and the InitialGuess is a value in between.

We also compared our algorithm to the one presented by Qian et al. [198] in terms of energy consumption and elapsed time. In short, the idea behind their state demotion inference algorithm is to use the RTT difference between states to infer the state transitions and therefore inactivity timers. Their algorithm sends a packet that triggers $\mathrm{PCH}-\mathrm{DCH}$ state transition and calculates the RTT by sending another packet after a number of seconds. They increase the number of seconds starting from 0 to a maximum and infer the inactivity timers from the measured RTT values. The elapsed time and the energy consumption of the algorithm is very dependent on the first guess of the maximum. It is clear that the maximum needs to be longer than the maximum estimate of the tails (larger than the sum of $T_{1}$ and $T_{2}$ ). We implemented their algorithm in Java using UDP packets. For comparing with our algorithm we used the same value for TestPeriod as their maximum, and started our UpperBound with TestPeriod. The average of 3 different rounds is calculated for each of the points in Fig. 5.4.

Fig. 5.4 shows that their algorithm results in larger elapsed time and more energy consumption. It can be seen that whereas the increase of consumed energy and elapsed time is somehow linear in their case, in our case it varies depending on the performance of the binary search (changing the maximum, we change the upper bound of the search). However, in the 
least favourable case, our algorithm consumes $65 \%$ less energy and takes 35 seconds less to converge on the estimated value for the timers.

Algorithm 2: We first ran the algorithm and inferred the thresholds. In order to verify them, we sent packets with the triggering sizes and observed the state transitions on the live power consumption trace generated by our measurement setup. The settings for the test were 1100 bytes for MaxSize, 50 bytes for MinSize and 10 bytes for MinDiff. LargeIncrement and SmallIncrement were set to 50 and 2 bytes respectively. $T_{1}$ and $T_{2}$ were set to 5. InterPacketInterval of Algorithm 2 was set to 1.5 seconds. Averaging 3 rounds of the running of the algorithm itself consumed 78 joules and took 97 seconds. Since no other algorithm is found, no comparison is performed.

To sum up, we have provided algorithms to infer the main parameters that impact the communication energy consumption. The next section presents our traffic scheduling algorithm which uses these parameters to schedule application background traffic.

\subsection{Cross-layer burst buffering algorithm}

The Cross-Layer Burst-Buffering (CLBB) algorithm builds on the concept of proportional computing. The goal of the algorithm is to map the appropriate utilisation level to the different states of the cellular interface, and thus employing the least consuming state when the utilisation is low is essential.

Regarding $3 \mathrm{G}$, this translates to minimise energy consumption by means of scheduling small data transfers in FACH state and avoiding costly state transitions to DCH. Transitions to DCH lead to energy overheads due to the $T_{1}$ and $T_{2}$ inactivity timers. In addition, the algorithm extends the idle time in $\mathrm{PCH}$ by not sending packets until the very last moment.

Our algorithm is divided in two parts: a packet driven mechanism that inserts application packets in two different queues depending on their size, and a UE state based mechanism that transmits the actual packets.

Let us define the problem as follows. Let $A_{f}=\left\{a_{1}, \ldots, a_{n}\right\}$ be the set of running foreground applications with strict time constraints (c.f., hard realtime) that perform data transmissions (e.g., user interaction). Let $T_{f}$ be the shortest relative deadline for each packet generated by an application in the set $A_{f}$. Let $A_{b}=\left\{b_{1}, \ldots, b_{n}\right\}$ be the set of applications that generate traffic with no time constraints (background traffic). We define $T_{w}$ as maximum waiting time for a packet transmission originated by $A_{b}$, which is the required time metric to perform the data transmission (note that all applications have such maximum time since otherwise one would just block the traffic). In practice, $T_{f}<T_{w}$.

We further classify the packets of the set of applications $\left(A_{f} \cup A_{b}\right)$ in two categories according to their size L (for large) and $\mathrm{S}$ (for short). Let $Q_{S}$ and $Q_{L}$ be queues of packets queued according to their size and sorted by inserting the shortest time to send at the front of the queue. Algorithm 1 and 
2 infer the inactivity timers $T_{1}$ and $T_{2}$, and the RLC buffer thresholds $B_{1}^{u}$ and $B_{2}^{u}$ (PCH-DCH and FACH-DCH uplink state transitions respectively). $T_{e}^{u}$ is the time to empty the RLC buffers in FACH which depends on the current data rate of the channel.

Let us elaborate on the time requirements for the packets. $T_{w}$ represents the time requirement of the background traffic, e.g., updates from RSS, email or Facebook applications. These can be set by the applications or based on user energy requirements (energy profiles). $T_{f}$ is defined as a deadline for other applications. Our algorithm only schedules the background data transmissions, i.e., the packets with a maximum waiting time and no strict time requirements. $T_{w}$ is in practice the maximum time that the algorithm can delay a packet transmission from p.t, i.e., the time that the packet was originated at the application.

Algorithm 3 describes the CLBB algorithm. The first part of Algorithm 3 (lines 1-8) simply queues the packets depending on their size sorted by shortest time to be sent $(t)$ after delaying their latest time to send by $T_{w}$. $Q_{S}$ contains packets that can be sent in FACH without triggering a state transition to DCH.

The main part of Algorithm 3 (lines 9-48) transmits data based on the UE state if there is any packet to be sent. The actions that the UE can perform are to transmit a single packet, transmit a queue in a burst or wait.

The UE starts from the PCH state and for every packet sending is able to distinguish between a state transition to $\mathrm{DCH}$ or $\mathrm{FACH}$ with the knowledge of the RLC buffer thresholds and and the inactivity timers $T_{1}$ and $T_{2}$. Every time a packet is sent it updates the time of the last transmission and the current state. After the inactivity timer duration the state of the UE is changed. Alternatively, the algorithm can employ the UE state provided by EnergyBox.

When the UE is in DCH we transmit the packets stored in $Q_{L}$ and $Q_{S}$. When the UE is FACH, it transmits the packets stored in $Q_{S}$ avoiding to trigger a costly state transition to $\mathrm{DCH}$, i.e., at limited data rate. This is done by sending a number of packets that sum up to less bytes than $B_{2}$. Then, the UE waits $T_{e}^{u}$ before the next sending of packets. This allows the UE to remain in FACH state until $Q_{S}$ is emptied.

Finally, when the UE is in $\mathrm{PCH}$, the algorithm compares the time to send $t$ of packets in the front of $Q_{L}$ and $Q_{S}$ and waits for the nearest time to send $T_{\text {nearest }}$. Next, the UE decides if it will send a packet or burst that triggers a state transition to $\mathrm{DCH}$ or $\mathrm{FACH}$. There are three cases.

First, in case the packet with the shortest time to send is in $Q_{L}$ (line 29 ), it needs to be sent. $Q_{L}$ will be transmitted triggering a transition to DCH. Second, if the packet is in $Q_{S}$, the UE will transmit $Q_{L}$ if the volume in bytes of $Q_{L}$ is larger than the PCH-DCH buffer threshold (line 31-34). This will lead to a trigger of a transition to $\mathrm{DCH}$ (note that once in $\mathrm{DCH}$ both queues are emptied).

In the third case, the transmission in $\mathrm{FACH}$ is feasible with the current 


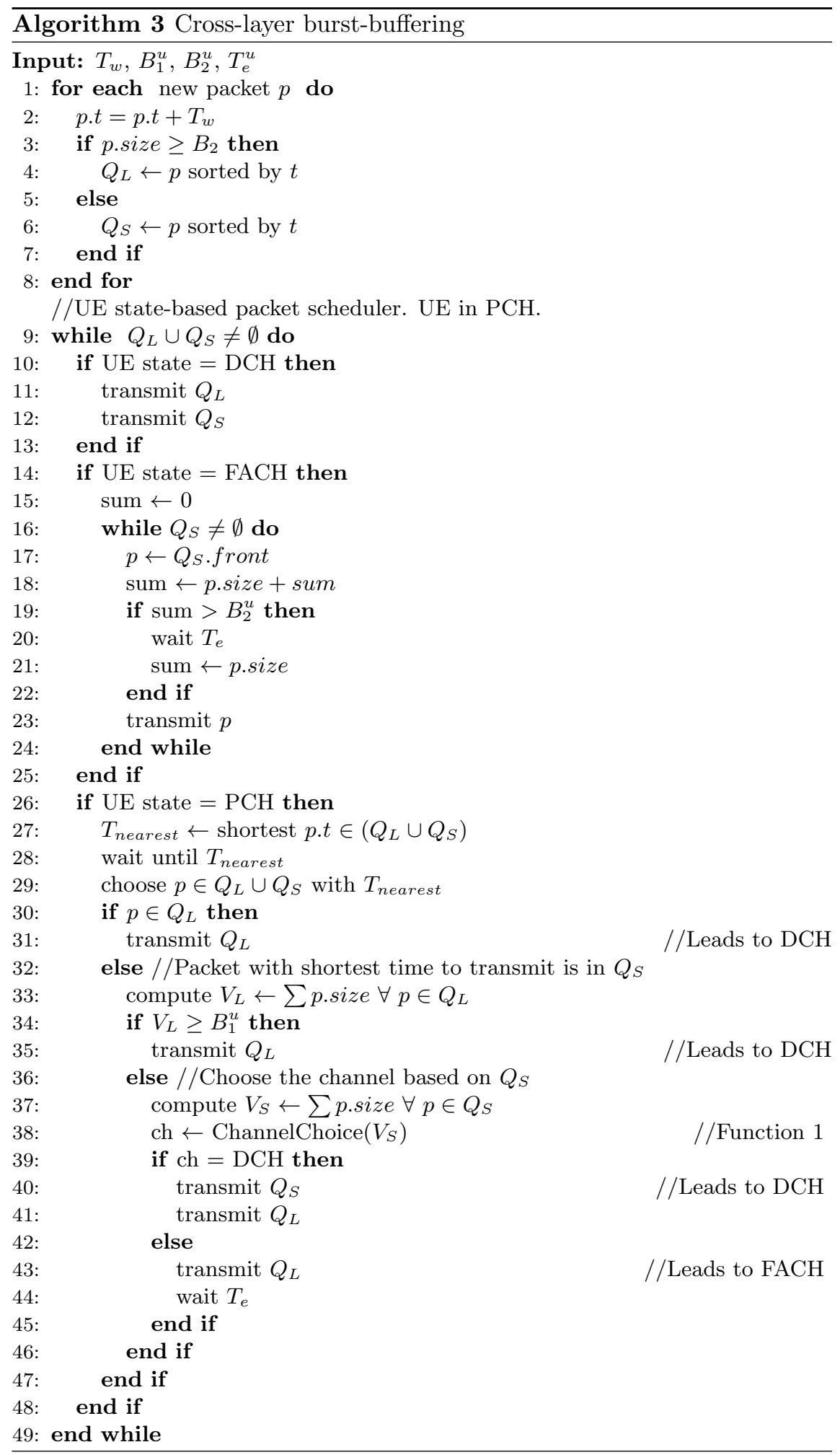




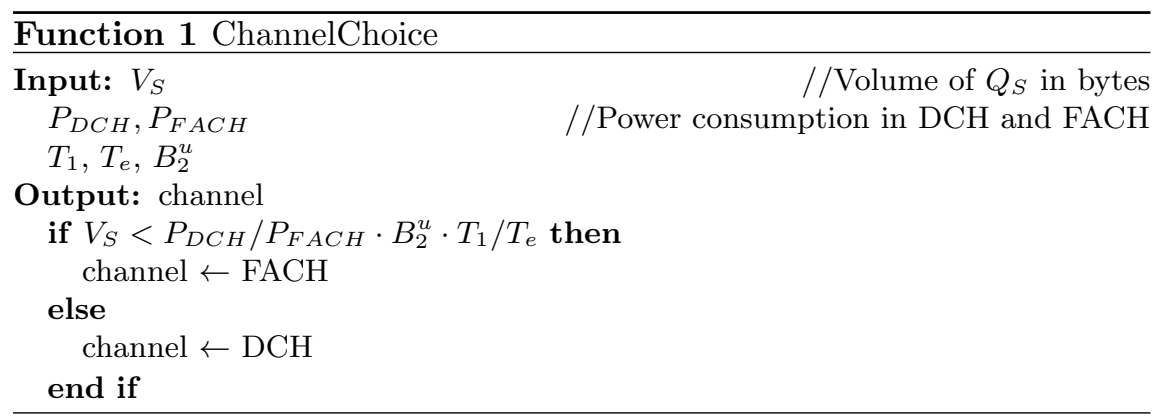

volume of the queues. However, a large volume of $Q_{S}$ can lead the UE to stay in FACH for a long period, which would consume more energy than sending the queues in burst in DCH. The choice of the less consuming option, i.e., sending on DCH or FACH, is performed by Function 1 (ChannelChoice) in line 37 given the volume of $Q_{S}$. If sending in FACH is less consuming, the UE will transmit $Q_{L}$ which triggers a FACH transition and wait $T_{e}^{u}$. $Q_{S}$ will be emptied in the FACH state. If sending in DCH is less consuming, the UE transmits both queues in burst triggering a DCH transition.

Function 1 is an optimisation to avoid the case when a large volume of data in $Q_{S}\left(V_{S}\right)$ leads the UE to stay in FACH for a long period. Given the power consumption of $\mathrm{DCH}$ and FACH $\left(P_{D C H}\right.$ and $\left.P_{F A C H}\right)$ and the transmission time $(\epsilon)$ of $Q_{S}$ in $\mathrm{DCH}$, the energy cost of transmitting in $\mathrm{DCH}$ is estimated by:

$$
E_{1}=P_{D C H}\left(T_{1}+\epsilon\right)+P_{F A C H} T_{2}
$$

Given the high data rate of $\mathrm{DCH}$, we consider that $\epsilon$ is negligible for the estimation. The energy cost in FACH is estimated by:

$$
E_{2}=P_{F A C H}\left(T_{2}+T_{e}^{u} \frac{V_{S}}{B_{2}^{u}}\right)
$$

Therefore, when the inequality $E_{1}>E_{2}$ holds, ChannelChoice will return FACH, otherwise DCH.

\subsection{Evaluation methodology}

In order to evaluate the total energy savings, we are interested in calculating the networking energy savings that the algorithm provides, as well as the energy cost of running the algorithm.

The broadband module development kit used to measure the $3 \mathrm{G}$ transmission energy (Appendix B) provides the most accurate evaluating method to quantify the networking energy savings by isolating communication energy. However, we cannot measure the overhead of running the algorithm using the development kit, and thus the algorithm was implemented in a commodity device to enable the evaluation of the energy cost of running it. 
The evaluation of the CLBB algorithm is performed in two different environments. The implementation of the algorithm in a handheld device provides the needed data to compute the total energy savings (networking energy savings minus the cost of running the algorithm). EnergyBox is conveniently employed to calculate the networking energy savings while a simple CPU model is derived to compute the CPU cost of running the algorithm. The second environment is used to compare the networking energy savings of the algorithm against the state-of-the-art performing physical measurements in the broadband module.

We begin this section by briefly describing the implementation of the algorithm in 5.4.1 and present the two evaluation environments and methodologies in sections 5.4.2 and 5.4.3. The evaluation results obtained with the first evaluation environment are presented in section 5.5 and finally section 5.6 presents the results of the networking energy savings of our algorithm measured in a broadband module.

\subsubsection{Algorithm implementation}

This section provides the details of an Android implementation of the CLBB algorithm as a Kernel Level Shaper (KLS) [219] in the Android platform. The implementation was developed by Joseba Sanjuan as part of his Master Thesis work supervised by the author.

The architecture of the KLS resides in kernel space since the throughput in kernel space is higher [131], and a user space application would require to interact with the kernel space through system calls. This would create a higher CPU footprint due to data transfer (read/write), e.g., a single data transfer can result in a CPU usage up to $33 \%$ utilisation [105]. The implementation creates a Netfilter Kernel Loadable Module (LKM) that can be inserted at runtime, instead of changing the native code at kernel compiling time. Netfilter is a Linux kernel framework that enables packet interception and modification at different hooks (decision points in the networking stack). The implementation registers the Netfilter NF_IP_POST_ROUTING hook in the IP layer. This allows us to intercept the outgoing packets without interacting with applications, leading to low CPU footprint. The architecture is deployed in a rooted Android device (HTC Sensation Z2710e). We cross-compile the Linux kernel with the needed options (e.g., enable module loading support and Netfilter), boot the new kernel in the device, crosscompile our KLM and load it at runtime.

\subsubsection{Methodology to assess the total savings}

This section starts by describing the data generation approach in order to evaluate the implementation of the algorithm. Then the methods to compute networking energy consumption and CPU consumption are described.

Data generation: In order to provide experiment repeatability and con- 
trol over the traffic generation of applications, real background packet traces were captured and replayed with and without the KLS in the Android device. We call this emulated traffic since we replay previously captured real packet traces. The choice of the emulated traffic is motivated by the need to create the same application traffic in various experiments to calculate the networking energy savings. These traces contain best-effort traffic that have no specific QoS requirements.

For the emulated traffic, tcpdump and an iptables based firewall were used to capture only the traffic from the chosen applications. Facebook, Skype and WhatsApp were used as test applications and all the traces were recorded with the screen switched off and without any user interaction. These applications created different traffic load over time based on their operation. Since the energy saving of the algorithm depends on the amount of traffic, out of the used traces we selected what we believe are representative traces for different traffic volumes and characteristics. The packet trace is replayed from an Android application sending uplink UPD packets to a server that replies with the downlink traffic. The traces are around 10 minutes long. Fig. 5.5 shows packet size, inter-packet interval (IPI) and the traffic volume characteristics of the traces. The name of the traces correspond to their traffic volume (low, medium and high). The traces have a majority of small packets and the transmissions range from regular (high) to sporadic (low) as the IPI shows in Fig. 5.5.
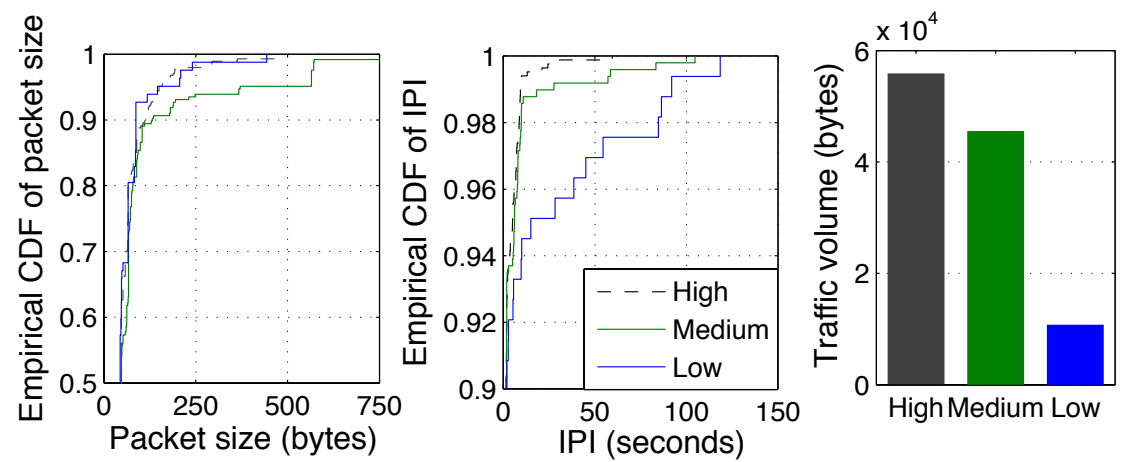

Figure 5.5: Characteristics of the emulated traces.

The traces described in Fig. 5.5 are used varying the maximum waiting time for a packet transmission $\left(T_{w}\right)$ of the algorithm (30,90 and $\left.180 \mathrm{~s}\right)$. For every trace, we replay the trace without KLS as baseline. Then, the KLS is loaded and the traces are replayed for the different $T_{w}$. We measure the energy savings and CPU usage of the KLS as described below.

Networking energy consumption: The communication energy consumption is conveniently calculated using EnergyBox. The $3 \mathrm{G}$ network settings we use correspond to the operator TeliaSonera shown in Table 3.1. We set the following power values for the different $3 \mathrm{G}$ states based on measure- 
ments for a mobile device [197]: $P_{D C H}=600 \mathrm{~mW}, P_{F A C H}=400 \mathrm{~mW}$ and $P_{P C H}=0 \mathrm{~W}$. By setting $P_{P C H}=0 \mathrm{~W}$, we aim to quantify only the energy spent in data transmission and energy tails.

CPU energy consumption: The CPU usage of the KLS itself is retrieved using the top utility. We perform physical measurements in the mobile device to characterise the power usage for different CPU loads in a very simplistic manner. The measurements are performed by replacing the battery of the smartphone by a low-side sensing circuit (described in Appendix $\mathrm{B})$. The frequency of the $\mathrm{CPU}$ is set to the maximum $(1.2 \mathrm{GHz})$ in order to measure the highest power level, i.e., worst case. This also simplifies the quantification of the CPU power usage by avoiding the power variations due to dynamic voltage and frequency scaling. A CPU stress application is developed to increase the CPU load step by step up to 100\% (7 steps). The $\mathrm{CPU}$ power is isolated from other factors such as screen, network interfaces or other applications by switching the components off and killing the processes that can interfere in the measurement. By interpolating the (CPU load, power) points we obtain a function that is employed to obtain the power consumption out of the CPU load data. The model used is presented in Figure 5.6

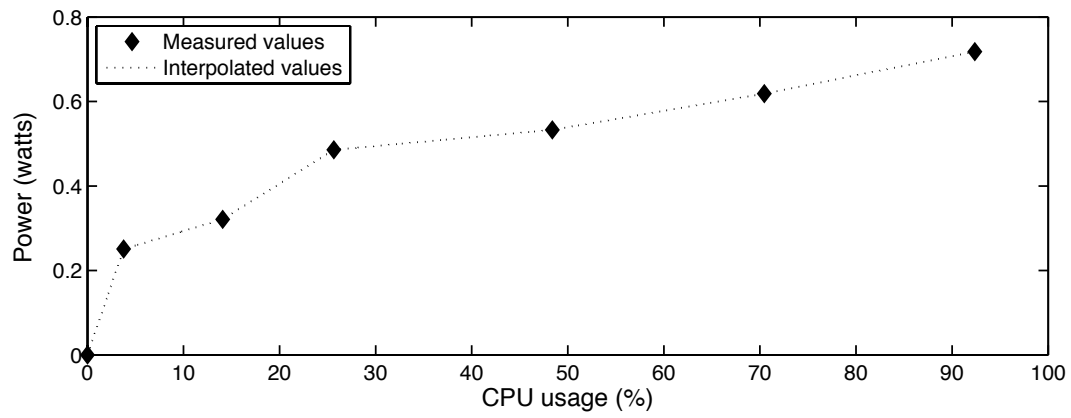

Figure 5.6: Measurement-based CPU model.

KLS settings: The settings for the KLS are set for the TeliaSonera network described in Table $3.1\left(B_{1}^{u}=1000\right.$ bytes and $B_{2}^{u}=294$ bytes $)$. We set a conservative value $T_{e}^{u}=300 \mathrm{~ms}$ to avoid potential FACH-DCH transitions. $T_{w}$ is varied for the different tests (30, 90 and 180 seconds). The inactivity timers are used to simulate the state of the UE.

\subsubsection{Algorithm benchmark methodology}

We evaluate the networking energy savings comparing them to TailEnder [24] as a baseline, the state-of-the-art algorithm to schedule background traffic. Directly measuring the communication energy on the broadband module provides a fine-grained evaluation environment. This section pro- 
vides a brief description of TailEnder, the data generation approach, the test framework used for the evaluation and a brief description of the algorithm implementations.

TailEnder: The algorithm schedules applications' data transmissions based on user-specified deadlines. Each transmission is deferred until its deadline unless it arrives within $\rho \cdot T$, a time fraction of the tail-time (i.e., inactivity timer) of the most recent deadline. The algorithm is parametrised by the constant $\rho \in[0,1]$ is a constant, which is set to 0.62 in their implementation. We use the same settings.

TestFramework: We developed a $\mathrm{C}++$ TestFramework which runs in the test computer of our physical setup in order to run any packet-driven algorithm. The application allows us to replay any captured data traffic trace and schedule the packet sendings to our $3 \mathrm{G}$ modem. The overview of the application flow is shown in Fig. 5.7 and is explained below.

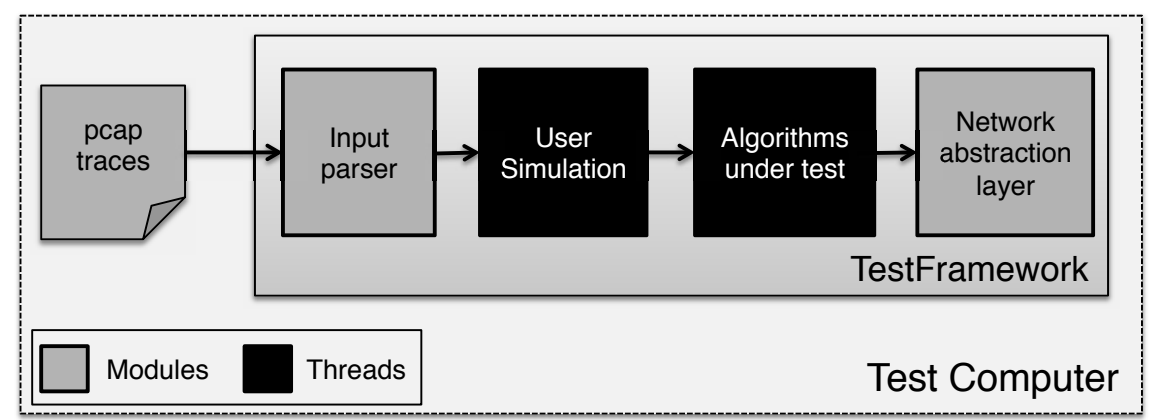

Figure 5.7: Overview of the TestFramework running in the test computer of the evaluation environment.

The pcap (packet capture) files consist of previously gathered data traffic packets. The input parser uses TShark [244] commands to convert the gathered pcap traces into sequence of packet sendings with the following format: \{timestamp, packetsize, packetnumber, ip_source, ip_destination, protocol\}. The user simulation thread replays the original trace providing the input packets to the interchangeable algorithm in turn. We implemented a simple packet forwarder (simulating FIFO), our CLBB algorithm and TailEnder as the algorithms under test. Finally, the network abstraction layer is a wrapper of network functionalities allowing the algorithms to send and receive packets to the server using simple functions.

TailEnder and CLBB in the TestFramework: Our algorithm is implemented in the TestFramework using two different threads (producerconsumer). The queues are implemented using C++ STL multimaps. Our TailEnder implementation is divided in two threads. The packet driven thread sends or queues a packet based on the following: if the UE is still within a time fraction of the tail time from the last sending it directly sends 
the new packet, otherwise the packet is queued. The other thread checks the shortest deadline of the queue and schedules transmissions when it is about to expire. Our implementation keeps track of the fraction of tail time by calculating the relative elapsed time from the last transmission.

Data generation: We collected a background trace of 40 minutes in a smartphone with the following applications running: Skype version 2.5.0.108 and Facebook 1.7.2. All the traces were gathered with the screen switched off and the application running in background. No user interaction was performed.

CLBB settings: Two different cellular operators are employed for this evaluation: TeliaSonera and Hi3g Access AB under the name 3 (operator 1 and 2 respectively). Table 5.1 shows the CLBB parameters for the two cellular operators. Operator 2 uses smaller RLC buffer thresholds and a longer $T_{1}$ than operator 1 , but it shows a shorter $T_{2}$.

Table 5.1: CLBB parameters for two different operators.

\begin{tabular}{|c|c|c|}
\multicolumn{1}{l|}{} & Operator 1 & Operator 2 \\
\hline$T_{1}(\mathrm{~s})$ & 4.1 & 8 \\
\hline$T_{2}(\mathrm{~s})$ & 5.6 & 2 \\
\hline$B_{1}^{u}$ (bytes) & 1000 & 90 \\
\hline$B_{2}^{u}($ bytes $)$ & 294 & 90 \\
\hline$T_{e}^{u}(\mathrm{~ms})$ & 300 & 600 \\
\hline$P_{D C H}(\mathrm{~W})$ & 1.3 & 1.2 \\
\hline$P_{F A C H}(\mathrm{~W})$ & 0.5 & 0.6 \\
\hline
\end{tabular}

\subsection{Energy simulation results}

The goal of this section is to calculate the total energy savings considering the networking energy savings and the cost of running the KLS (i.e., the implementation of the CLBB algorithm) embedded in a mobile device.

\subsubsection{Network communication energy savings}

In this section we validate the energy savings of the KLS in the mobile device for the 3 representative background traffic traces (High, Medium and Low) described in section 5.5. The replay of the captured trace without employing KLS is referred to original trace and used as the baseline for calculating the percentage savings on the y-axis in Figure 5.8.

Fig. 5.8 shows that the energy savings depend on the traffic volume: when the traffic volume is higher, the energy savings tend to be higher. The original traces consume 225, 161 and 105 joules for the high, medium and low traffic volume traces respectively. Moreover, as expected, increasing $T_{w}$ 


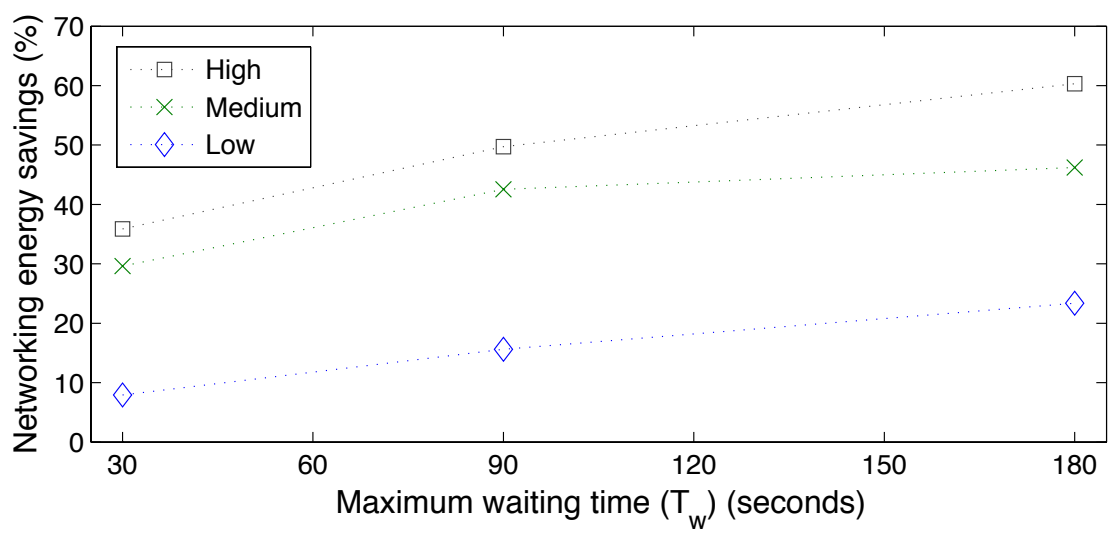

Figure 5.8: Network communication energy savings.

leads to higher energy savings. For the low traffic volume trace, the energy savings range from $8 \%\left(T_{w}=30 \mathrm{~s}\right)$ to $23 \%\left(T_{w}=180 \mathrm{~s}\right)$. However, the energy savings are much greater $\left(35 \%\right.$ when $\left.T_{w}=30 \mathrm{~s}\right)$ with more frequent background traffic. The maximum registered is $60 \%$.
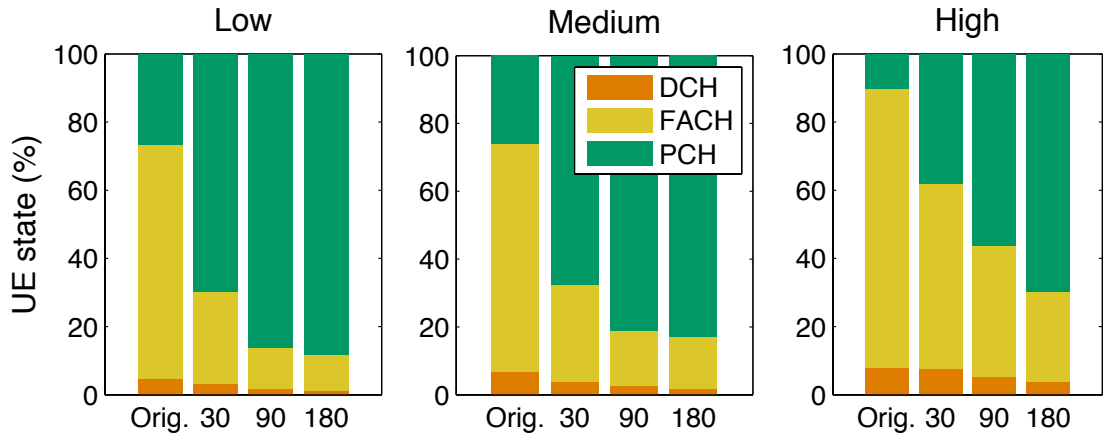

Figure 5.9: Percentage of time spent by the UE in the different $3 \mathrm{G}$ states over the total time of the original traces (y axis) scheduled with different $T_{w}$ (seconds) by the KLS (x-axis).

Fig. 5.9 shows the percentage of time spent by the UE in the different $3 \mathrm{G}$ states over the duration of the original traces compared to the case where the traffic is scheduled by the KLS. The KLS extends the time spent in PCH state which leads to energy savings. Intuitively, a greater $T_{w}$ makes the UE to be in $\mathrm{PCH}$ for longer period. In general, the amount of time in FACH is reduced in all the cases. For the original traces, it can be noticed that the UE spends more time in FACH with higher amount of traffic. Regarding $\mathrm{DCH}$, the UE performs occasional burst transmissions with the High and Medium traffic profile, leading the UE to $\mathrm{DCH}$ for a short time period. However, with Low traffic profile, the UE rarely transitions to DCH. 
To summarise, we validate that the KLS implementation of the CLBB algorithm provides high energy savings to the UE.

\subsubsection{CPU energy footprint}

In this section we show the CPU energy cost of running the KLS. The implementation performs two main operations, intercepting and queueing the packets, and sending them (dequeueing and re-injecting them in the network stack). The rest of the time the threads of the KLS are sleeping and therefore do not waste CPU time and energy.

In order to quantify the energy footprint when queueing packets, we queue several UDP packets from an application and observe the CPU load created by the KLS. The CPU usage of the KLS is logged and the energy footprint is obtained from the CPU load employing our CPU power characterisation explained in section 5.5. Our measured CPU load is below $0.01 \%$ which shows that the footprint due to the queuing operation is negligible in terms of CPU and therefore energy consumption.
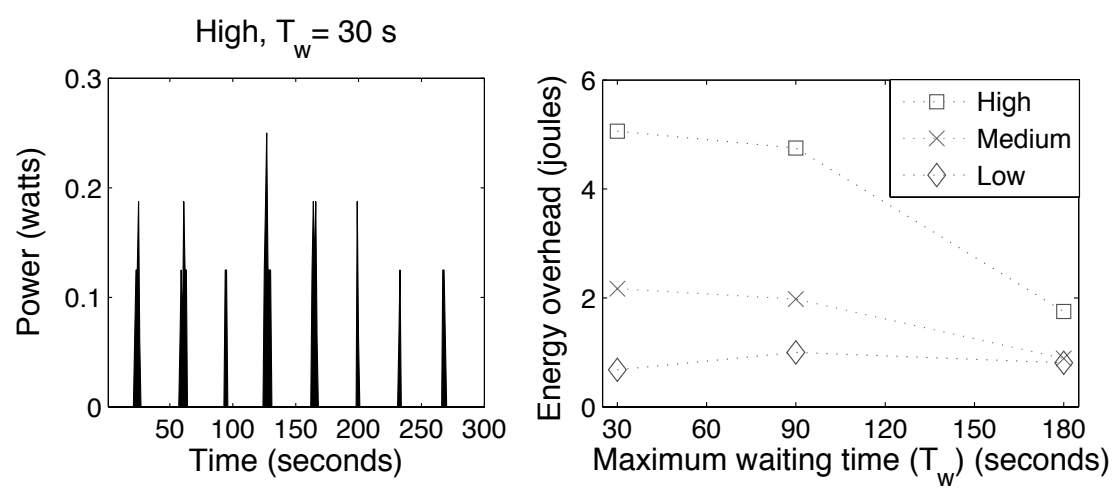

Figure 5.10: Example of CPU energy consumption by running the KLS (left) and CPU energy footprint for the different traces (right).

Next, the CPU energy footprint due to sending the packets is measured for the high, medium and low traffic volume traces. Fig. 5.10 (left) shows an example of the energy consumption when running the KLS for $T_{w}=30$ s for the high traffic volume trace. The KLS only creates CPU load spikes that are lower than $5 \%$, which consume less than $0.25 \mathrm{~W}$ as it is shown in Fig. 5.10. These power spikes do not consume much energy.

Fig. 5.10 (right) shows the additional energy consumption of the CPU for the traces varying $T_{w}$. The maximum energy cost is 5 joules, which is only $2 \%$ of the energy consumed for sending the original high traffic volume trace ( 225 joules as shown in section 5.5.1). The energy footprint is higher for traces with higher traffic volume since the KLS has to process more packets. Except for the case of low traffic volume, a greater $T_{w}$ leads to lower energy consumption. 
To summarise, we see that the maximum energy footprint of the KLS depends on the amount of traffic and $T_{w}$, but it is low compared to the energy used to send the original traffic due to energy-efficient kernel implementation of the algorithm.

\subsubsection{Total energy savings}

This section presents the total energy savings, i.e., the network transmissions savings minus the CPU energy cost of the KLS, i.e., the overhead. Since the energy cost of the KLS is small, the energy savings still remain high.
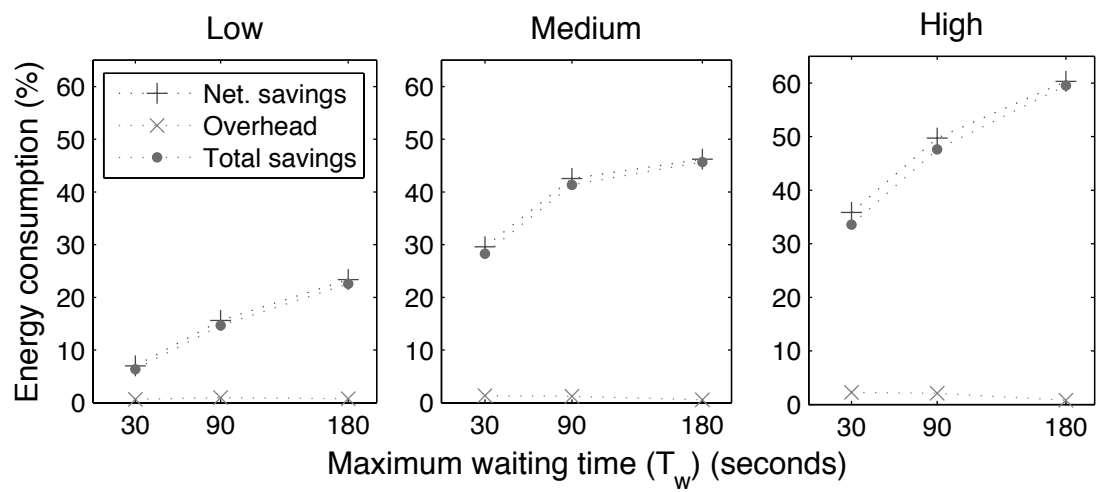

Figure 5.11: Network transmissions energy savings, KLS energy overhead and total energy savings as a percentage of no-KLS base energy consumption.

Fig. 5.11 shows the networking savings of the KLS, the energy cost of the KLS and the total energy savings as a percentage of the total energy consumption of the original traces $(225,161$ and 105 joules for High, Medium and Low traces respectively). When the traffic is low or moderate, the overhead is low and the total energy savings remain close to the networking energy savings. A somewhat higher energy overhead is observed for the High data profile, but still the total energy saving is significant. In this case, the highest saving is achieved with the highest $T_{w}$, leading to a $58 \%$ net saving.

\subsection{CLBB vs. TailEnder}

We now focus the study in thoroughly comparing the networking energy savings against the state-of-the-art algorithm using the broadband module for two different networks.

In order to calculate the energy savings, we measure the energy consumption baseline by transmitting the original trace gathered from the Android smartphone using the TestFramework. Then, for each of the points in Fig. 5.12 and 5.13 we ran our TestFramework with the original trace as input 
and measured the energy consumption using TailEnder and our algorithm separately. To compare the algorithms, $T_{w}$ is set to be the user specified deadline of TailEnder and it is equal for all the packets. The energy savings are computed with respect to the baseline varying the maximum waiting time $T_{w}$ for the packets.

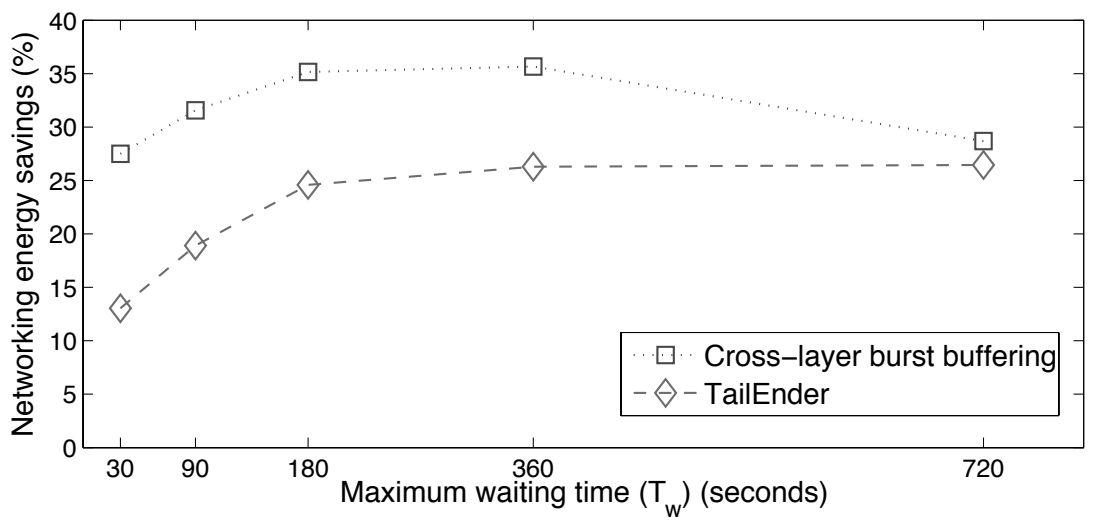

Figure 5.12: Energy savings of CLBB and TailEnder for operator 1.

Fig. 5.12 shows that the energy savings of the cross-layer scheduler are higher than the TailEnder. The energy consumption baseline was 777 joules of the original trace. The minimum energy savings in our measurements are $27 \%$ for this particular trace. It is worth mentioning that for the shortest $T_{w}$ our method achieves higher networking energy savings than larger $T_{w}$ for TailEnder.

The higher energy savings for the short $T_{w}$ of our method in comparison to TailEnder are achieved based on the fact that transmitting small packets in FACH is more energy-efficient than triggering DCH state transitions. Our method takes advantage of the small packets avoiding the unnecessary state transitions to DCH.

For TailEnder, the larger the $T_{w}$ value the higher energy savings can be achieved. Since the traffic aggregation is performed with more data packets, the UE stays longer in the PCH state. The same issue arises for the crosslayer burst buffering when increasing the $T_{w}$. However, the energy savings of our method decreases for applications with longer $T_{w}$. The cause of this fact is that the probability of having bigger packets in the queues increases when increasing $T_{w}$ and therefore the possibility of performing transmissions in $\mathrm{FACH}$ is lower.

The gap in Fig. 5.12 can further increase by network settings that have greater $B_{2}^{u}$ or shorter $T_{e}^{u}$, i.e., higher available data rate in FACH. Further, data sets with smaller packets would be more beneficial to our system as well as operator settings with a longer $T_{1}$.

Operator 2 employes smaller $B_{2}^{u}$ and allows lower data rates in FACH. Fig. 5.13 shows the networking energy savings results for operator 2 (base- 
line 1120 joules). Even though operator 2 has a very small $B_{2}^{u}$ and larger $T_{e}^{u}$, the energy savings of CLBB are still higher since it still performs transmissions in FACH. The gap between both algorithms is decreased since the value of $B_{2}^{u}$ allows fewer transmissions in $\mathrm{FACH}$ and the data rate in $\mathrm{FACH}$ is lower.

The energy savings provided by the algorithm compensate for the cost of the algorithms to infer the network parameters. This cost of was measured to be 95 joules in section 5.2.3, which is already compensated in the 40 minutes trace of the test.

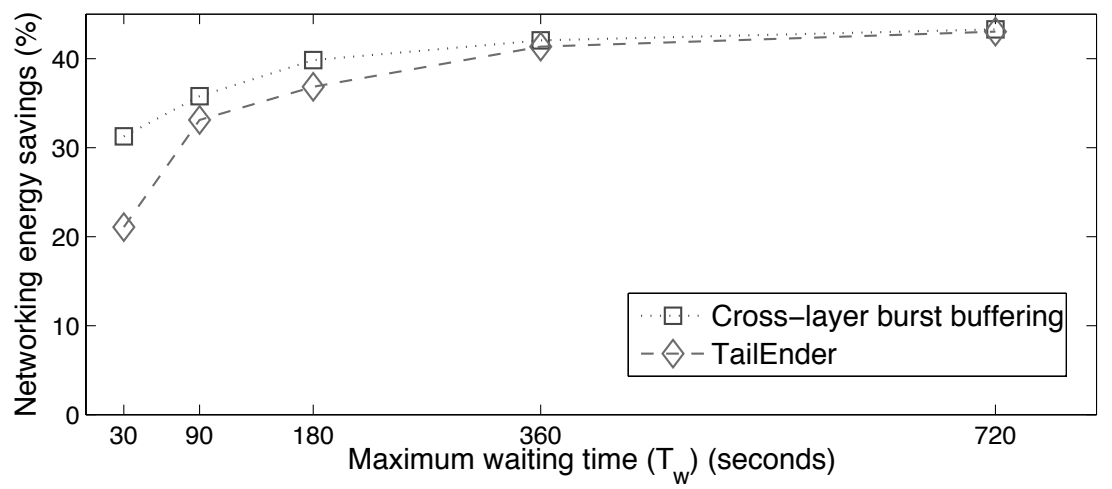

Figure 5.13: Energy savings of CLBB and TailEnder for operator 2.

Finally, Fig. 5.14 shows a fragment of one of the runs of CLBB using the network of operator 1 for $T_{w}=90$ seconds. The example shows that by aggregating transmissions CLBB keeps the interface in idle until a packet needs to be sent. Instead of transmitting the aggregated packets in a burst that would lead to DCH, the example illustrates how the transmissions are performed in the FACH state reducing the energy consumption due to the wireless interface.

\subsection{Discussion}

Achieving energy proportionality in systems that were designed for peak performance is a complex task. The focus of this chapter was on developing a generic energy saving solution that can provide energy proportionality during low utilisation periods given aggregated flows from different applications with no QoS requirements. These transmissions allow to alter their data pattern in order to create energy-efficient traffic.

The chapter also has shown that EnergyBox is indeed a valuable tool for providing energy awareness as well as evaluating energy-efficient solutions. EnergyBox has efficiently quantified the networking energy savings of the proposed solution. In this context, the information of the radio communication layer has been included as part of our solution: our scheduler uses a 

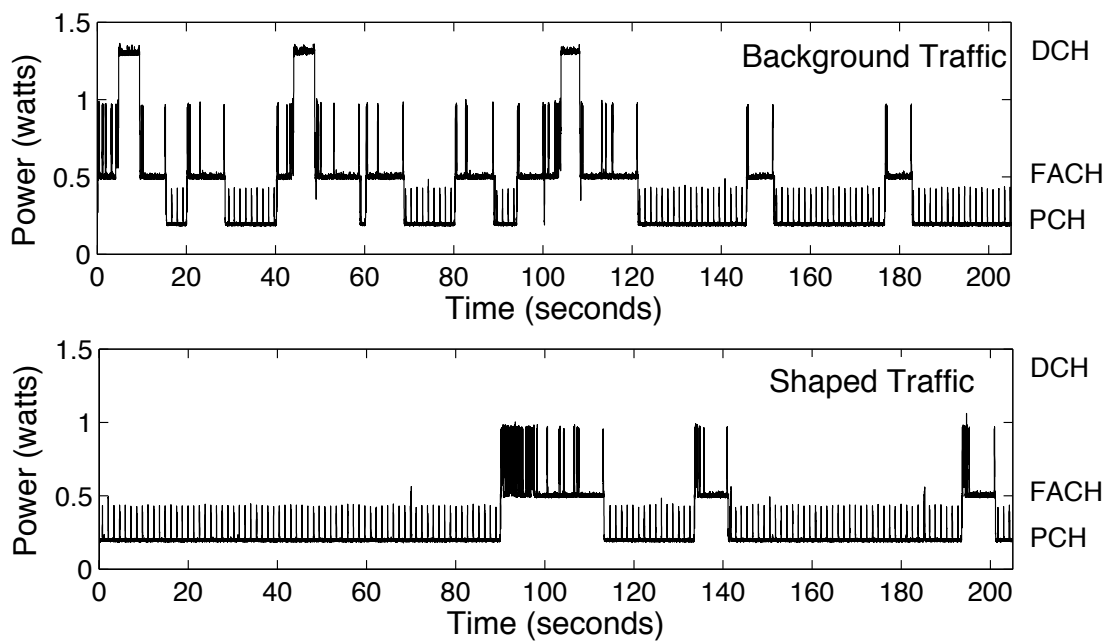

Figure 5.14: Energy consumption examples of background traffic and energy-efficient shaped background traffic.

more detailed radio communication layer knowledge to improve the energy savings results of the state-of-the-art. Moreover, we study the cost of running the solution itself and show that applying a mechanism that employs a small amount of energy to save energy pays off.

The maximum time to wait for a transmission is employed by the algorithm to delay transmissions. The parameter can be provided by applications (or users through the applications) or the operating system. While our evaluation used several values ranging from seconds to minutes, this can be adjustable by the user as a trade-off between energy and delivery delay. If a user has been idle for some time it is more likely that the user will continue inactive [75], and thus the maximum time to wait could be increased using inactivity time as an estimator. Mining common user communication can also improve the approach.

In this work we do not consider fragmentation, application layer information nor the use of the radio link quality estimator like RSS to adapt our data transmissions. Packet fragmentation would allow to reduce the packet sizes to be transferred in lower consuming states and application traffic statistics and historical data could be employed to improve the traffic aggregation. Considering link quality would further increase the energy savings by communicating during good link periods when the communication is less costly.

The question of traffic differentiation is an open field for further exploration. Applications (and therefore developers) could be in charge of setting different priorities to different transmissions or differentiating the background traffic from the rest. Thus approaches to efficiently and intuitively provide this alternative to applications and support it is an interesting 
investigation. However, great part of the background traffic is created by applications due to not being aware of the communication energy characteristics. Traffic differentiation without application cooperation is an interesting approach to investigate, which could be performed at different layers to maximally exploit the energy saving potential.

For traffic types other than background, energy-efficient solutions need to consider the requirements of the traffic or applications, which implies that the traffic data pattern cannot be completely modified. In the following chapters we study different tailor-made solutions to reduce the energy consumption of some applications informed by their usage context. 
"Every minute you spend in planning saves 10 minutes in execution; this gives you a 1000 percent Return on Energy!"

Brian Tracy

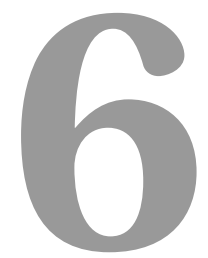

\section{Energy-Efficient Location Sharing}

Reducing $E(\{i\})$ can often improve the energy efficiency of a system. However, it does not always necessarily reduce the total energy consumption of the system $E(N)$ since the interaction between the different entities in the system might not be efficient. It is often the case that an entity has a limited or no control over the interaction with the other entities, and thus reducing $E(\{i\})$ becomes the main approach to improve the energy efficiency of the system from an entity perspective.

In our context, developers or the application itself can maximally influence $E(\{i\})$ by quantifying and studying the energy consumption the application in isolation with tools such as EnergyBox. In this chapter we study how the energy consumption of a specific application type can be reduced at the design stage by performing the correct choices that impact the energy consumption through the data pattern (e.g., protocols or payload size). Hopefully, the domain knowledge of certain application categories, the user usage and the generated data pattern enables the creation of tailor-made energy-efficient solutions.

We focus on location sharing services which are provided by applications integrating geographic location with services, such as navigation systems, sport trackers or business information delivery. There are countless applications and services that support the user for sharing their location data with other users (e.g., Glympse, Foursquare, Gowalla or the original Google Latitude now included in Google+), also referred to Location Sharing Applications (LSAs).

Fig. 6.1 shows an overview of data flow for a typical LSA, where the location data is often shared over cellular networks. Earlier studies have 
shown that the use of a mobile device's battery for frequent transmissions of position data in a LSA can be more expensive than the energy spent on location retrieval itself [120]. This is in part due to energy-agnostic application development and in part dependent on the choices that impact the data pattern (e.g., protocols or data format).

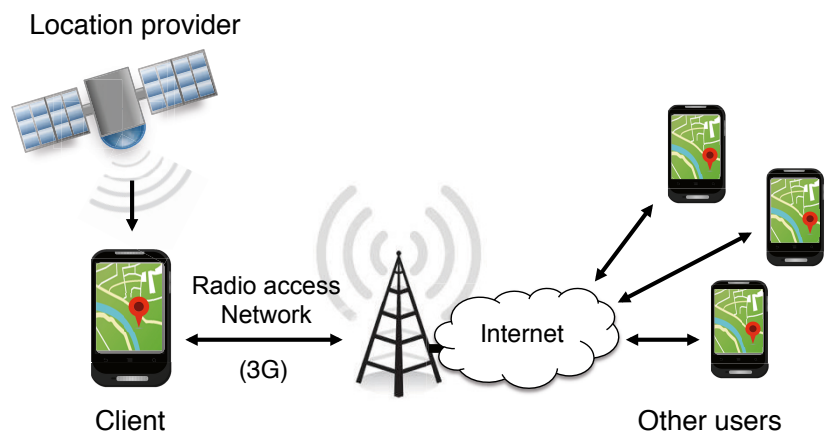

Figure 6.1: Overview of the data flow in a location sharing service based on cellular (3G) communication.

To explore the influence of potential protocols for location sharing on energy consumption, we compare two different approaches which represent two potential options for a developer while developing an application. Given usage data we also suggest a protocol choice based on the results of the comparative study. Finally, we briefly analyse the impact of a more compact data encoding format on energy consumption. The implementation of a LSA developed for the Master thesis of Mihails Prihodko [188] is used to experimentally collect real data and perform the comparative study. This chapter is mostly based on the material from that thesis which was performed under author's supervision.

In this mobile scenario EnergyBox facilitates the energy consumption evaluation by simply collecting the communication traces in the client smartphone with no additional equipment, where the portability of the physical measurement equipment and isolating the data communication from the rest of the energy consumption would have been an obstacle.

The chapter is organised as follows: The scenario and the developed LSA for experimentally comparing the protocols is presented in section 6.2 . The evaluation settings are described in section 6.2 , and the results are presented in sections 6.3 to 6.5 . The results are discussed in section 6.6.

\subsection{Scenario setup}

Since current location sharing applications are closed systems (products), we develop a prototype test application designed to share location information 
among different users connected to Internet via a UMTS network. The location sharing application (LSA) consists of a client side and a server side that can communicate with two different protocols representing application layer design options. TCP is used as transport layer protocol for both techniques. The server side simulates location updates of the friends of the user.

Protocol selection: We select the lightweight Message Queuing Telemetry Transport protocol (MQTT) as an application layer protocol. The MQTT protocol is a simple publish-subscribe messaging protocol designed for machine-to-machine (M2M) or "Internet of Things" contexts, e.g., constrained devices used in telemetry applications [157]. The messages are sent in a push-oriented manner, similar to other technologies such as Google Cloud Messaging, Apple Push Notification Service or cloud push notification services (e.g., Pushapp).

We also select the standard Hypertext Transfer Protocol (HTTP) as one of the most common client-server protocols. HTTP or newer protocols such as Bidirectional-streams Over Synchronous HTTP (BOSH) or WebSockets can be used in different ways in order to achieve bi-directional communication (e.g., HTTP server push or private push services). However, we choose the standard form of HTTP for its pull-oriented nature.

The selection of the protocols is not critical since the aim of the study is to show the methodology to improve the energy consumption of an application in isolation and the practicality of EnergyBox. Other protocols can be added to the comparison as future work. The two protocol alternatives are summarised below:

- HTTP technique: Since HTTP functions as a request-response protocol using the client-server paradigm, the client polls the server in order to retrieve other clients' location information updates. A textencoded location object is sent between the client and the server.

- MQTT technique: The publish/subscribe paradigm implemented by MQTT is used as an alternative protocol for location sharing services. The location data is a text-encoded object sent between the publisher/subscriber and the MQTT broker. The broker is a piece of software in the server side that matches publisher messages to subscribers.

Client-server side interaction: In the client side implemented in the mobile device, the GPS location provider creates an event roughly once every second while the device is moving. The sharing period can be chosen independently from the location provider update period, that can depend on current application requirements such as accuracy or energy efficiency [120]. The location sharing update period is denoted by $T$.

Fig. 6.2 shows how a single location sharing update is performed for both techniques. Whenever the client updates its location using HTTP, it also retrieves other users' location updates. The location update of the 


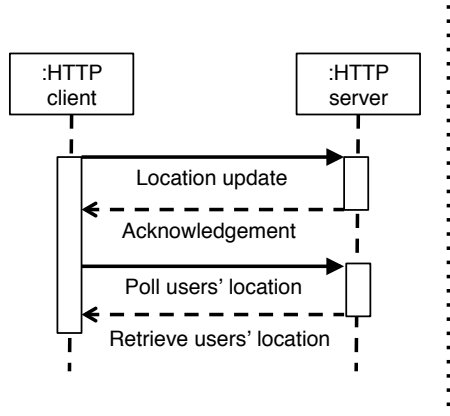

(a) HTTP

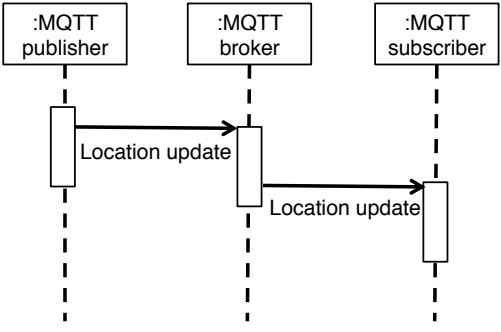

(b) MQTT

Figure 6.2: Location Sharing Application interaction.

publisher is instantly forwarded by the broker to the subscribers when using MQTT. Since the HTTP technique polls the server to retrieve users' location updates, the locations are sent in batches. The MQTT broker forwards the published updates to the subscribers as soon as they reach the broker.

User simulator: It is hosted in the server side and simulates the location sharing activity of any number of (other) users in our experimental evaluation. The simulator is designed to mimic the behaviour of users constantly moving, i.e., updating their position periodically once every $T$.

Since the HTTP technique polls the server to retrieve users' location updates, the locations are sent in batches. The user simulation creates a new location for every user once every $T$. The default behaviour of the MQTT technique is different since the broker forwards the published updates to the subscribers as soon as they reach the broker. The simulated publishers send their location update once every $T$ using a random offset to mimic real user location sharing behaviour.

Location data and encoding: We selected a subset of the values provided by the Android location object based on the study of the API of a popular application ${ }^{1}$, and included time, latitude, longitude, speed, accuracy, altitude and bearing in our location object. Since the data encoding influences the data pattern in terms of packet size, we also consider different data encoding formats in our study. JavaScript Object Notation (JSON) is a text-based human-readable format used as de facto standard for mobilebased applications.

In the search of more compact formats, we also encoded the location data in Base64 Content-Transfer-Encoding format (base64). Base64 is a format designed to represent arbitrary sequences of octets in a text-based non-human readable form. The sizes of the different types of formats are 154 and 61 bytes for JSON and base64 respectively.

\footnotetext{
${ }^{1}$ Google Latitude API
} 


\subsection{Evaluation settings}

Analysis of the energy consumption, assisted by EnergyBox, allows exploration of the design space for reducing the energy footprint of a LSA. The LSA architecture is used varying the number of users in the system (different network load), location sharing update interval $(T)$, and data encoding.

Data collection: We perform our experimental study by using an Android smartphone (LG P990) with a GPS receiver as a UE connected to a $3 \mathrm{G}$ network of the TeliaSonera operator in Sweden. The experiments emulate real user location traces performed walking outdoors while carrying the smartphone with GPS signal over a predefined path at the university campus. The idle experiments were performed indoors where no GPS signal is available and the smartphone is stationary to avoid location updates. We simulate a number of users with whom the client shares location updates using the user simulator hosted in the server or broker for HTTP and MQTT respectively. The length of each experiment is 10 minutes. An iptables based firewall is employed to allow only the traffic created by the LSA. We capture the data traffic of the LSA using tcpdump in the smartphone, which is later used to quantify the energy consumption using EnergyBox.

LSA settings: The location sharing update interval $(T)$ is increased in multiples of 15 seconds $(15,30,45$ and $60 \mathrm{~s})$ to study the impact of the update frequency on the data traffic and energy consumption. In order to study the impact of higher traffic load, the number of simulated users (active friends sharing their location data) in the system is set to $0,3,6$ or 9 . The settings for the data encoding formats are specified for each experiment.

Communication energy: The communication energy is calculated from the collected traces using EnergyBox with the settings shown in Table 3.1. The UE power values for the different RRC states are based on earlier measurements [197]: $P_{D C H}=800 \mathrm{~mW}, P_{F A C H}=460 \mathrm{~mW}$. We set $P_{P C H}=0 \mathrm{~W}$ in order to quantify only the energy spent in location sharing communication. Since EnergyBox provides repeatable estimations for a given packet trace, we conveniently run each packet trace only once.

Evaluation metrics: Similar to previous evaluations, we employ the total energy consumption and total percentage of time spent by the UE in different RRC states over each experiment time as evaluation metrics. We also study the total amount of data sent over the experiment.

\subsection{MQTT vs. HTTP}

In this section we compare the HTTP and the MQTT techniques using the same data format (JSON), update period and number of simulated users. We differentiate the idle state (no location updates by any of the users in the system) from the active state. 


\subsubsection{Idle state}

The UE may spend significant part of its time in idle state between aperiodic or infrequent location sharing updates (e.g., check-in mode or infrequent events). Fig. 6.3 shows the energy consumption and amount of data traffic generated by the HTTP and MQTT techniques in the idle state, where no location data is exchanged. The data traffic and energy consumption values are normalised to the greatest values, $18.59 \mathrm{kB}$ and 143.37 joules respectively.
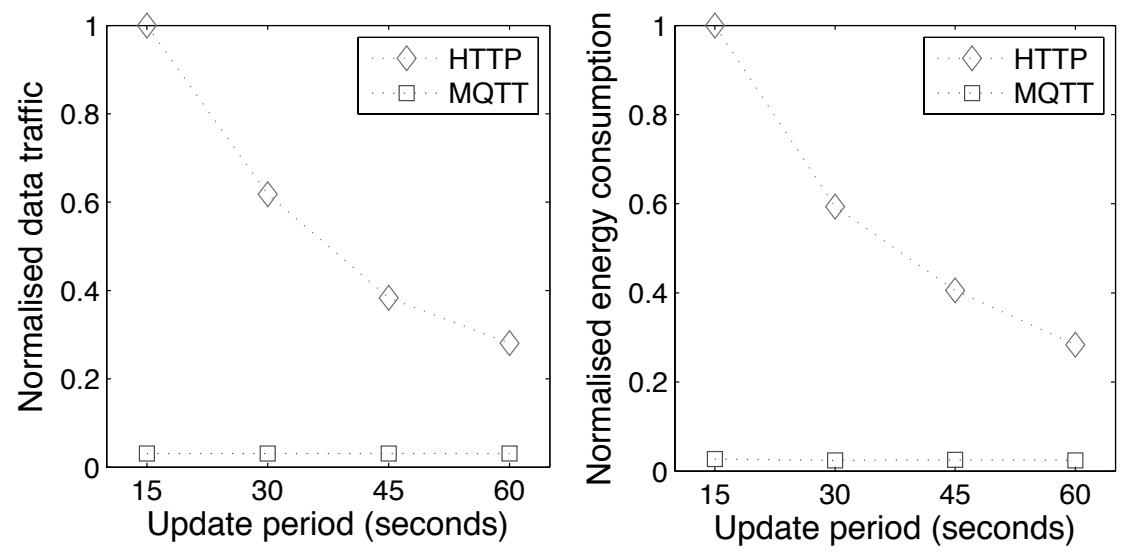

Figure 6.3: Amount of data traffic and energy consumption for HTTP and MQTT techniques in the idle state.

As expected, the HTTP (pull) technique creates more traffic than the MQTT (push) technique. The update period $T$ strongly influences the data traffic for HTTP, which gradually decreases when increasing $T$. The main benefit of the publish-subscribe paradigm employed by MQTT is that the broker can send a location sharing update whenever it happens without polling the server, irrespective of the update period.

Fig. 6.3 also illustrates that the HTTP technique consumes more energy than the MQTT technique. The HTTP client needs to poll the server even when no new location data is available leading to energy waste. The longer the update period $T$, the smaller the energy consumption becomes. However, the delay between the users' location updates and the reception of them becomes greater.

Since the HTTP transmissions do not contain any location data, the data only triggers the FACH state and makes the UE consume one energy tail. The energy consumption of MQTT is constant. The UE only switches to $\mathrm{FACH}$ for the connection establishment and then spends the rest of the experiment in $\mathrm{PCH}$. 


\subsubsection{Active state}

In the idle state experiments the energy was mostly consumed by polling or preparedness to send. We now study the behaviour of HTTP and MQTT when actual location data is transmitted. The goal of the experiments is to understand the operational needs of the application so that the most energyefficient protocol can be chosen by the service developer according to the requirements. We vary the update period $T$ and the number of simulated users that the client shares location data with, i.e., the load in the system. The case of 0 simulated users represents when the client updates its own location, sharing with no friends.
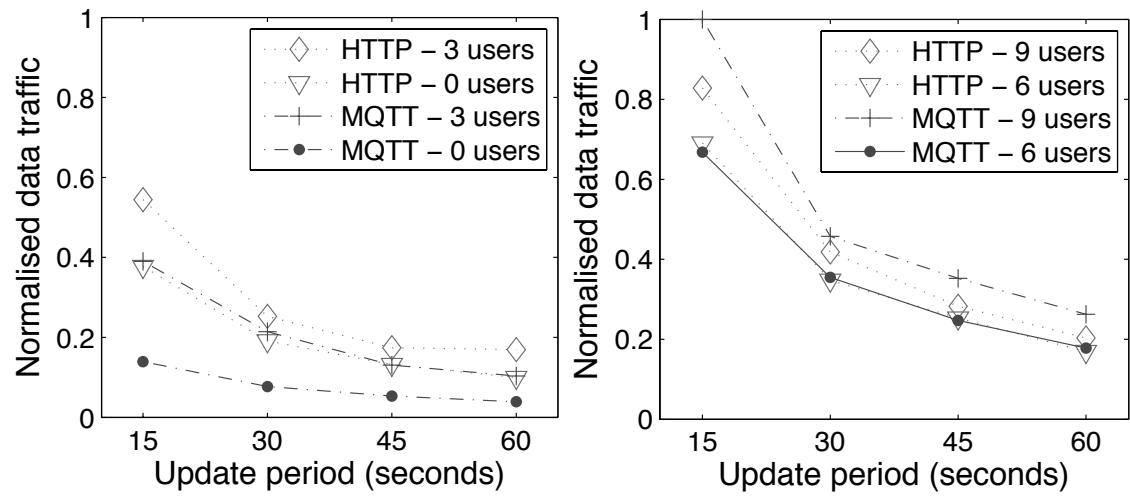

Figure 6.4: Amount of data traffic for the HTTP and MQTT techniques in active state.

Data communication: Fig. 6.4 shows the amount of data transmitted normalised to the greatest value $(125.24 \mathrm{kB})$. We split the results into two figures for readability. Fig. 6.4 (left) shows that when the number of users is low MQTT generates lower traffic load compared to HTTP, independently of the update period. The small overhead of the MQTT protocol is the main differentiating factor for low number of users. A single location update performed by the client consists on average of 421 bytes and 1159 bytes, for using MQTT and HTTP respectively.

Fig. 6.4 (right) shows that the MQTT and HTTP techniques generate similar amounts of traffic when the number of users is 6 . Somewhat surprisingly, the MQTT technique creates more traffic for 9 users compared to HTTP. HTTP retrieves all the location updates in bundles every update period, whereas the MQTT publish/subscribe updates are transmitted whenever they happen. Sending the updates in small packets separately increases the protocol data overhead. For example, the many small packet transmissions of MQTT result in a the total TCP overhead of $23 \%$ for the case with 9 users and an update period of 15 seconds, whereas it is only $3 \%$ for the HTTP technique as a result of bundling the updates.

Fig. 6.5 shows the packet size and inter-packet interval (IPI) distribu- 

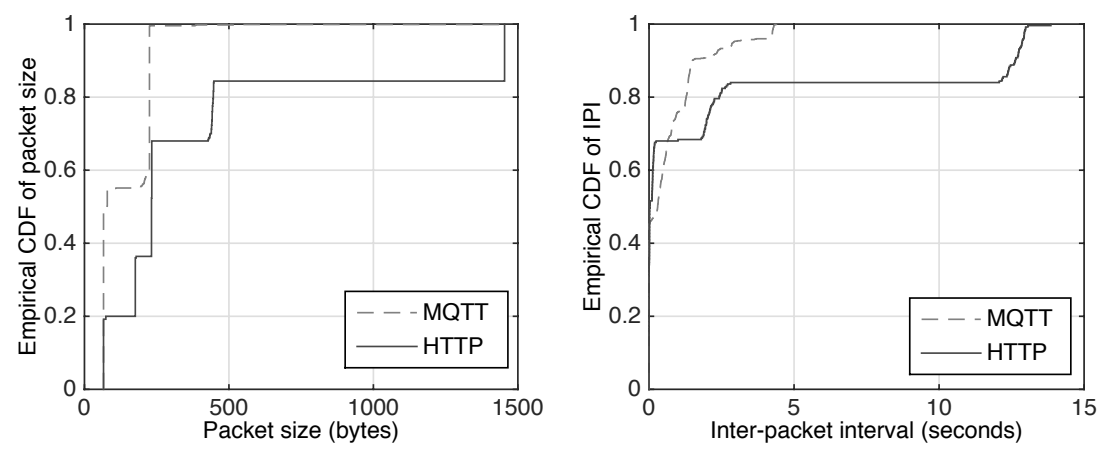

Figure 6.5: Empirical CDF of packet size and inter-packet interval of HTTP and MQTT (9 users and $T=15 \mathrm{~s}$ scenario).

tions for the case with 9 users and an update period of 15 seconds. Even with the same data encoding, the packet size for the MQTT technique is smaller as it is shown in empirical cumulative distribution function (CDF) of the packet size in Fig. 6.5 (left). Fig. 6.5 (right) shows that the IPI is typically shorter for HTTP. Most of the packet transmissions of the HTTP technique are bursty transmissions involving acknowledgements with short IPIs, whereas for MQTT the packet transmissions are more sparse. The other factors that impact the empirical CDF of HTTP are the PCH-DCH transition delay and the update interval $T$. Note that the MQTT technique is not affected by the $\mathrm{PCH}-\mathrm{DCH}$ transition delay since the transmissions are mostly performed in FACH (the $\mathrm{PCH}-\mathrm{FACH}$ transition delay is much shorter than the PCH-DCH transition delay).

Energy consumption: The communication energy footprint for the HTTP and MQTT techniques is shown in Fig. 6.6 normalised to the greatest value (285 joules for the case of MQTT with 9 users). For HTTP, we show the energy consumption when only the user uploads her location data ( 0 users) and the average when there are more users in the system. The energy consumption of HTTP is approximately the same for 3, 6 and 9 users, and therefore we only show the average value to compare with MQTT (see "HTTP - with users" in Fig. 6.6). Since the amount of data transmitted every update period using HTTP is always greater than the PCH-DCH RLC buffer threshold, the UE is moved to DCH. Hence, the number of energy tails caused by the inactivity timers dominate the energy consumption. Therefore, the longer the update period the lower the energy consumption.

For 0 users (when only the client updates it own location regularly), the energy consumption of the HTTP and MQTT techniques are similar. Fig. 6.6 shows that the MQTT technique is more energy-efficient for shorter update periods when the number of users is 3 . When the update period is greater than 3 users, the HTTP technique consumes less than the MQTT technique since it shares the location with all the users once every $T$. The 


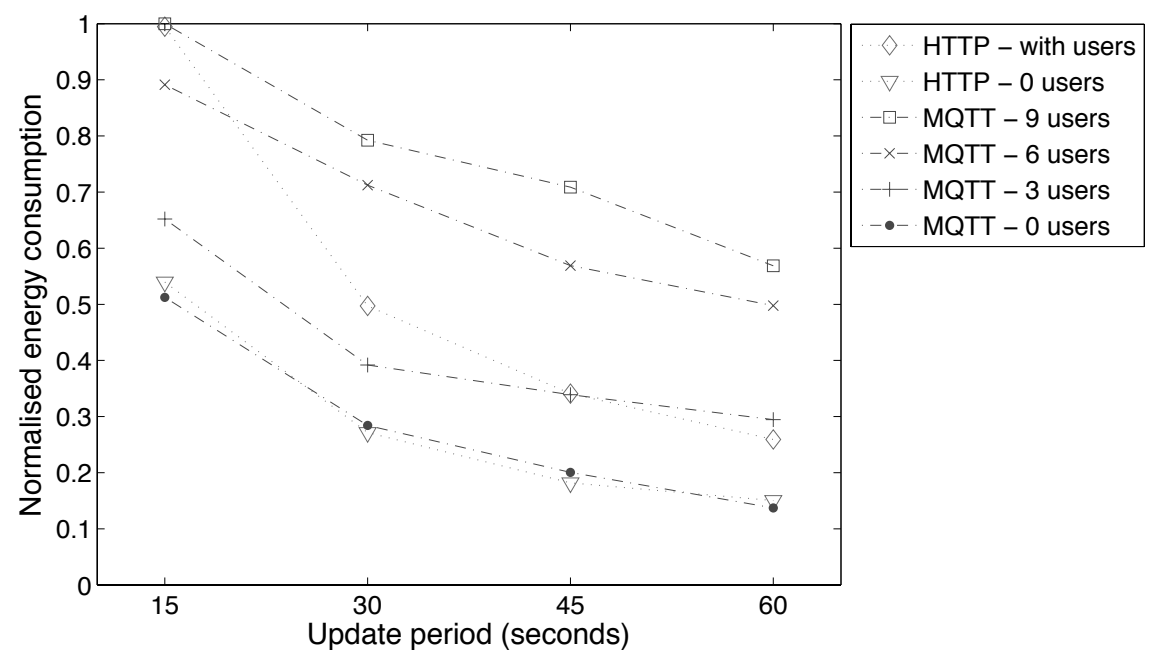

Figure 6.6: Energy consumption for the HTTP and MQTT techniques in active state.

HTTP technique becomes more efficient when the number of users increases (6 and 9). The reason is that HTTP transmits all the data in a single burst (pulling nature), at the cost of location sharing delay (every $T$ ).

RRC state occupation: Fig. 6.7 shows the fraction of time spent by the UE in the different RRC states over the total experiment time. Both techniques have a similar radio resource utilisation with 0 users. The UE performs transmissions mostly in $\mathrm{FACH}$, leading to the $\mathrm{FACH}$ energy tail after every update. When the client employs the HTTP technique with more users in the system, the transmissions are performed in $\mathrm{DCH}$ and the UE experiences the overhead of the DCH and FACH energy tails. Instead, the MQTT technique makes an efficient use of the FACH channel due to its low overhead while there are few users ( 3 user case). However, when the number of updates is increased, the FACH occupation is increased as well (6 and 9 users). The higher volume of location updates forces the UE to move to DCH using the MQTT technique.

The above charts demonstrate the usefulness of EnergyBox to perform efficient energy studies for location sharing services. EnergyBox facilitates the choice of protocols for applications by easily quantifying the energy consumption. The results show that the low protocol overhead and the publishsubscribe nature of MQTT makes the MQTT technique more energy-efficient in idle state and when the number of users is low. When the load is increased, the HTTP technique takes advantage of aggregating all the location updates in a single burst being more energy-efficient. However, this comes at the cost of delaying the reception of the updates at the client side.

Given the application usage (e.g., number of expected users or expected 

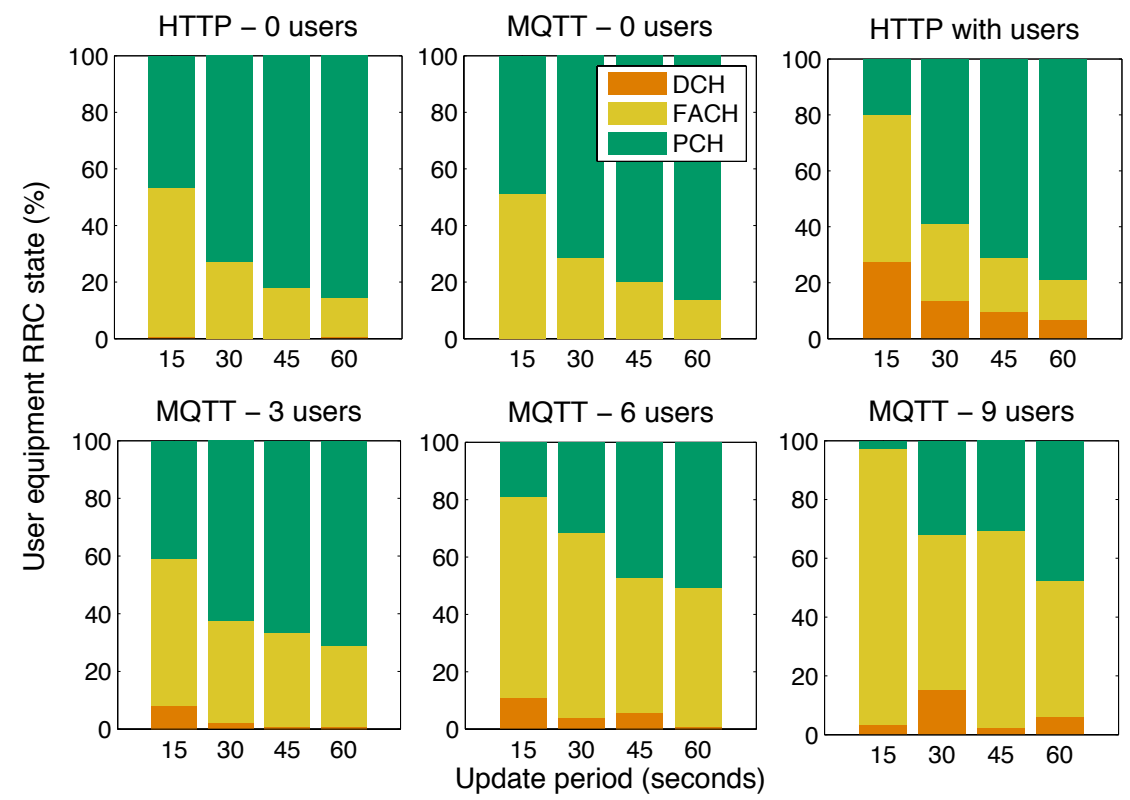

Figure 6.7: Percentage of time spent by the UE in the different RRC states over the experiments for HTTP and MQTT.

update period) the choice by developer of the service can greatly reduce the energy consumption at the user-end. According to our results, selecting an inappropriate technique can increase the energy consumption of the application by up to $112 \%$ (the case of 9 users in MQTT compared to HTTP). Systematically studying the energy consumption with EnergyBox may open up for avoiding such energy waste.

\subsection{Protocol for check-in}

We are interested in studying the time between updates (inter-update interval) for each user as an input to guide choosing between the two protocols. In this section we study the appropriate protocol for check-ins, i.e., the process whereby a user reports her presence or arrival at a location.

We employ the check-in dataset provided by Cheng et al. [47]. The dataset contains $22,387,930$ check-ins from 224,804 location sharing service users gathered from the public Twitter feed between September 2010 and January 2011. The check-ins dataset contains check-in information in tuples of $\{$ userID, TweetID, Latitude, Longitude, CreatedAt, Text, PlaceID $\}$ and user information $\{$ userID, StatusCount, Follower sCount, FriendsCount $\}$. We calculate the inter-update interval for each user using the above dataset. 

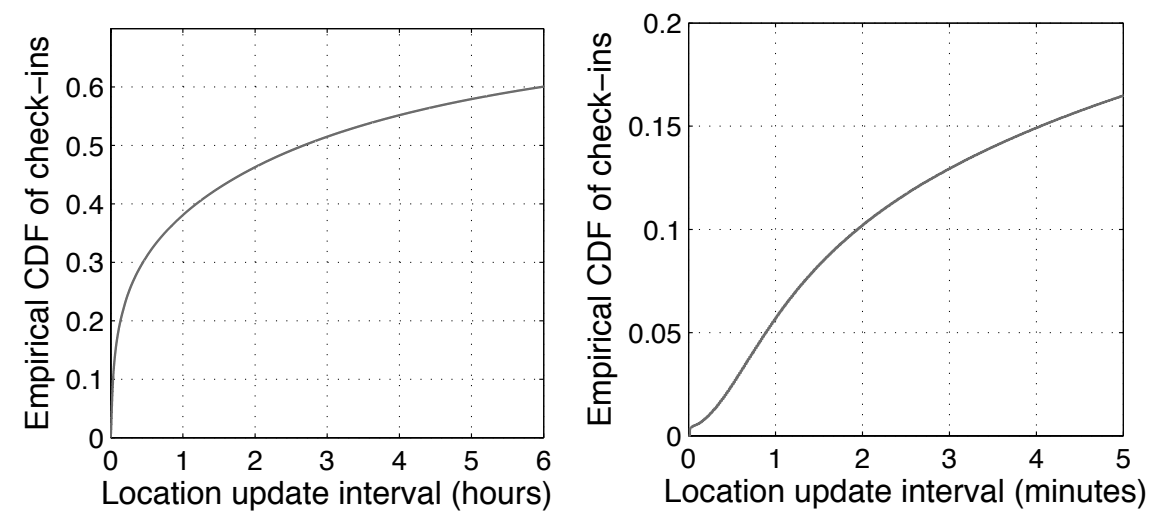

Figure 6.8: Empirical CDF of inter-update interval in hours and minutes of the $22,387,930$ check-ins from 224,804 users.

Fig. 6.8 shows the empirical CDF of the inter-update interval of all the users in the dataset. Fig. 6.8 (left) shows a general view in hours and Fig. 6.8 (right) describes the same empirical CDF for shorter location update intervals in minutes. The inter-update interval is long for check-ins, which makes the idle time to dominate the active time. It also presents a long tail (not fully shown in Fig. 6.8) that describes the sparsity in the check-ins of many users. Fig. 6.8 (right) shows that inter-update intervals shorter than 1 minute represent $5.6 \%$ of the total number of check-ins, and a single user rarely has more than 1 short consecutive updates per 1 minute (93\% of the users do not have any consecutive update shorter than 60 seconds).

In the previous section we saw that when the traffic load is small, MQTT was more energy-efficient than HTTP and resulted in similar energy when the period increased. Therefore, given that the majority of check-ins show long inter-update intervals, MQTT appears to be the protocol of choice due to its instant location update delivery and similar energy footprint.

\subsection{Data encoding impact on energy}

Earlier, we stipulated that communication energy of an application is greatly dependent on the data pattern. We now return to the data pattern in terms of packet sizes in the context of the LSA. The data encoding format impacts the size of the payload, and therefore the data pattern in terms of packet size. We now quantify the benefit of using a compact data encoding format (base64) for the HTTP technique over the standard verbose format (JSON). The comparison is performed using the scenario with 3 users, emulating the case where the client is to be notified of three friends locations continuously.

Fig. 6.9 (left) shows the energy consumption for both data formats normalised to the greatest value (283 joules). The energy consumption of 


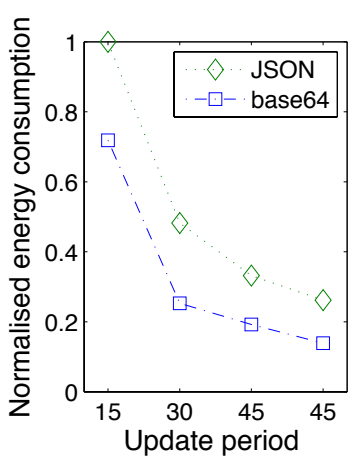

(seconds)

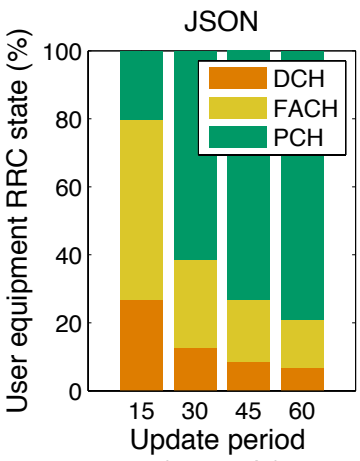

(seconds)

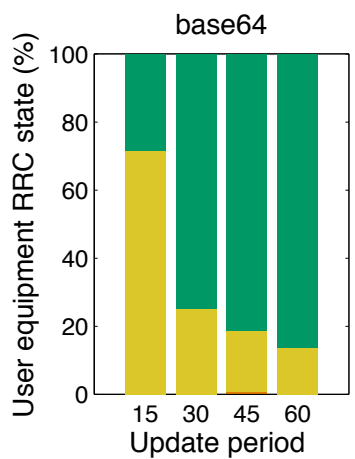

(seconds)

Figure 6.9: Energy consumption (left) and user equipment RRC states for different data encodings (right).

HTTP is significantly reduced when using a more compact format. The location sharing is performed in a single burst of packets in both cases. However, the reduced size of payload stops the UE from moving to DCH (Fig. 6.9 (right)), whereas the data transmissions of HTTP with JSON are performed in $\mathrm{DCH}$ (Fig. 6.9 (centre)). A single big packet is enough to trigger the FACH-DCH state transition based on the settings of the RLC thresholds, which leads to the $\mathrm{DCH}$ and $\mathrm{FACH}$ energy tails.

The results obtained with the EnergyBox show that the energy consumption can be reduced by 16 to $50 \%$ by using a compact data encoding format at the cost of losing human readability.

\subsection{Summary and discussion}

In the search for tailor-made energy-efficient solutions for application data communication, different design choices need to be systematically studied. We have shown that application protocol or data format choices impact the data pattern of the application, which in turn have a substantial impact on energy consumption.

As an example to illustrate such choices, this chapter compared the MQTT and HTTP protocols as suitable location sharing protocol choices over 3G networks. The publish/subscribe nature of MQTT ensures instant delivery of location updates, whereas a HTTP client needs to poll the server to retrieve updates creating a delay that is dependent on the update period. We show that MQTT creates less data traffic, is more energy-efficient in the idle state and when the number of users with whom the client shares its location is low. When the number of simultaneously sharing users increases, HTTP becomes the preferred option since the location updates are aggregated when the client polls the server. Our analysis shows that MQTT appears as the protocol of choice for check-in mode. Other use cases (e.g., 
continuous location tracking) expose an energy-delay tradeoff.

A further aspect that impacts the data pattern in terms of packet size is the data encoding format. Our results show that the energy consumption when using HTTP is reduced if the location data encoding format is compact.

Based on the presented results, the current work can be extended by developing an adaptive protocol switch (MQTT to HTTP) or switching the protocol mode (aggregating location updates for MQTT when the load is high) based on the number of users in the system to reduce their energy consumption. The energy overhead of switching the protocol mode, the location sharing delay and the energy savings would be interesting to study in a real application.

To sum up, this chapter has shown that reducing $E(\{i\})$, i.e., energy consumption of an application in isolation, depends greatly on developer choices at design stage which need to be informed in terms of energy consumption. Quantifying the energy consumption of different alternatives can support the selection of the most energy-efficient options, which in turn may contribute to reducing the energy consumption of the system. EnergyBox or similar energy simulation approaches can become valuable instruments in this respect to quantify $E(\{i\})$. In addition to this chapter, the next chapter studies how to reduce $E(\{i\})$ by knowing the general usage pattern of the application and quantifying application-specific mechanisms. 
"Typing..."

Unknown

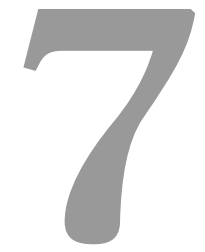

Energy-Efficient Instant Messaging

In this chapter we further explore means to lower the energy consumption of applications in isolation, i.e., $E(\{i\})$, now in the context of Instant Messaging (IM). In this type of application user interaction directly impacts system utilisation and energy consumption. Thus we show how the common utilisation knowledge can reveal inefficiencies and propose a simple solution to reduce $E(\{i\})$. We also argue that the energy consumption of additional features and mechanisms need to be quantified to further analyse their added value in terms of QoE compared to their energy footprint.

IM applications have overtaken the traditional Short Message Service (SMS) in total number of messages sent per year ${ }^{1}$. These applications usually offer the possibility of sending text messages (1-to-1 or to a group) and other multimedia messages for free (e.g., images, audio or video) as well as provide more functionalities than regular SMS, such as online presence awareness, typing notification or status updates.

Application developers integrate additional features without knowing the a posteriori impact on energy consumption. Recall from section 2.1 the great diversity of energy consumption of the different IM applications tested when sending the same conversation. We argue that the energy consumption of the added features need to be studied at the design stage in order to be aware of the correct operation choices (e.g., parameters) and the potential impact on the energy consumption, and therefore QoE. EnergyBox is a suitable tool to perform such studies.

Unfortunately, when the communication pattern is mostly determined by user interactions, the complexity of designing energy-efficient communication increases. This is very obvious in the case of IM applications, 
where interactive traffic is generated by a sequence of messages exchanged by users in a conversation. Traffic generated by IM applications is usually elastic, since no QoS support is assumed for delivering the text messages to the users.

IM messages are an example of a type of traffic with low bandwidth requirement, but they lead to high energy consumption due to the radio resource allocation of cellular networks. As previously discussed, the low utilisation and high energy consumption in a system shows a low energy proportionality. For example, the exchange of a couple of IM text messages can consume as much as sending an image which presents higher utilisation. From the cellular network operator perspective, the signalling overhead created by IM is very high given their intermittent and small data transmissions.

Knowledge of the user interaction pattern and its impact on the data pattern can enable the design of tailor-made energy-efficient solutions. Usage patterns can reveal inefficient communication since neither the users nor the applications are aware of the energy footprint characteristics.

In this chapter we collect, analyse and provide an IM text message dataset of 1,043,370 messages from 51 mobile users that describes users' diverse usage patterns. Informed by the user usage patterns found in our dataset, a message bundling algorithm is proposed showing that aggregating consecutive messages from the same user saves up to $43 \%$ energy. The suitability of using EnergyBox to quantify the energy consumption of different application features is demonstrated showing that typing notification, a network functionality most IM applications implement, amounts to additional energy consumption between $40-104 \%$ in isolation. While presence updates have been already studied in desktop-oriented machines [265] and in the mobile context $[51,149]$, the cost of the typing notification feature used by most chat applications has been neglected.

The chapter is organised as follows. Section 7.1 analyses the IM dataset of real user messages. Section 7.2 describes the proposed message bundling technique, followed by the algorithm applied to the typing notification feature in section 7.3. The evaluation environment is presented in section 7.4, and section 7.5 and 7.6 evaluate the energy savings of aggregating messages and the energy cost of the typing notification functionality respectively. Finally, the results are summarised and discussed in section 7.7.

The development of the IM application and the experimental tests reported in this chapter were performed by Simon Andersson as a Bachelor thesis [12] under the supervision of the author.

\footnotetext{
${ }^{1}$ http: //www.bbc.co.uk/news/business -22334338
} 


\subsection{Collected IM dataset}

Analysing the way users write text messages using IM applications can reveal current inefficiencies in terms of energy consumption. We are interested in the user input, which is translated to network traffic by the applications. Thus, we study a dataset of user text messages collected at the application layer in this section.

The messages were collected from one of the most widely used IM applications (WhatsApp) during a period between 23rd of January 2011 and 8th of January 2014. Note that the logs from different users have different starting time. We agreed on collecting the usage data with the users after they were created (from past logs), thus the data reflects the normal behaviour of the users. The users employ WhatsApp as their primary IM application.

A parser was developed to obtain the messages from the WhatsApp logs in each user device. Every text message is represented by its timestamp (UNIX time), the message length in characters, the direction (in/out), the user number, the chat type (single chat or group chat) and the chat number. A chat is a sequence of messages exchanged with a user (1-to-1 single chat) or a group of users (group chat) over the duration of collection. By conversation we will denote a subsequence of all the messages belonging to one of these chats. The user name and the actual content of the message are obfuscated for privacy reasons. The focus of this work is on text conversations, and therefore we do not consider multimedia messages, which are left for extensions of this work.

The dataset currently contains 1,043,370 messages collected from 51 users. The age of the users is different: 34 users between 25-30 years, 12 users between 30-35 years, and 5 users above 45 years. Regarding the country, most users are from Spain (33) and Sweden (13), whereas the rest are from Belgium (2), Germany (1), USA (1) and Mexico (1). The messages appear in 1815 conversations with other users (2089 users in total).

While the selection of representative subset of all messaging patterns in the world would require a careful analysis, we believe that the current dataset provides an interesting subset since (1) it has a diverse user base, and (2) it shows a great diversity in terms of number of messages sent per day as well as used chat types (single or group chat).

The timestamp and the message size are the most interesting values from the communication pattern perspective. We start by analysing the distribution of the messages according to their origin (in/out) and conversation type.

\subsubsection{Message origin}

Single chats are the most common type of conversation (83\% against $17 \%$ for group chats). The larger fraction of messages are originated from single chats ( $59 \%$ of all the messages), whereas the rest (41\%) is from group chats. Table 7.1 shows the number of messages per chat type and direction. It 
shows that the input messages dominate in the dataset being $65 \%$ of all messages.

Table 7.1: Message origin per direction and chat type.

\begin{tabular}{|c|c|c|}
\hline & In & Out \\
\hline Single chat & $29 \%$ & $30 \%$ \\
\hline Group & $36 \%$ & $5 \%$ \\
\hline
\end{tabular}

We observe that there is a great diversity in the above numbers across the different users of the dataset. Fig. 7.1 shows the message classification per user (the users are sorted by the number of single chat messages).

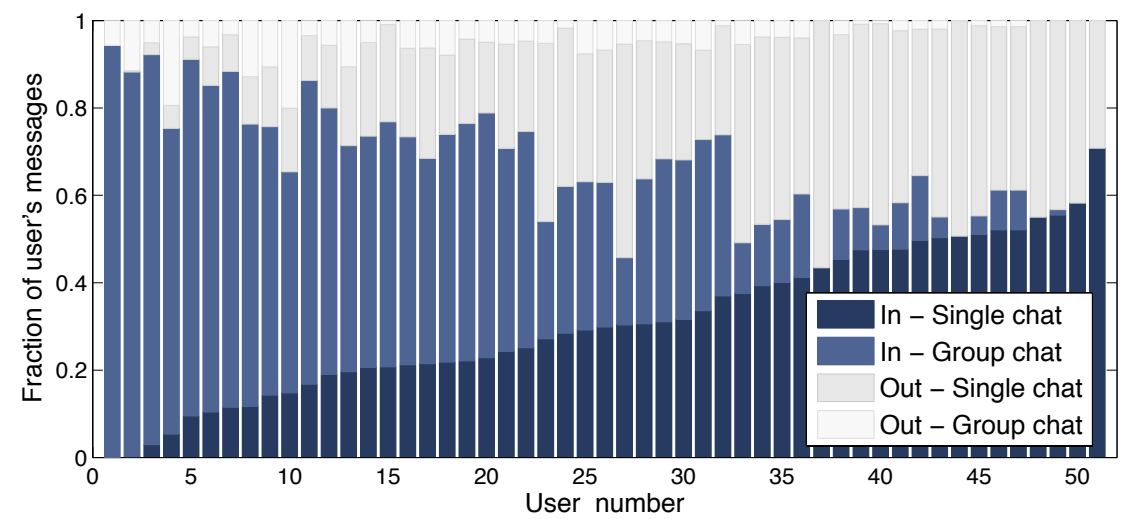

Figure 7.1: Message classification per user.

Most of the users are shown to have all the message classes, but in different proportion. For example, user 10 has a much larger proportion of group chat input messages compared to user 40 (50\% against 5\%), meaning that half of the messages received by user 10 are from group chats. However, user 40 has a larger proportion of single chat in and out messages, meaning that the user mostly uses single chats. Some users do not even use group chats (e.g., user 37 or 44).

The average number of users in group chats is 7 . However, larger groups are also present (up to 26 users) but less common. Only $11 \%$ of the groups are larger than 12 users.

Regarding the number of chats per user, the average and variance are $35 \pm 23$ chats. For an arbitrary user, the greatest proportion of messages is typically concentrated in few chats. On average, $50 \%$ of the messages of a user belong to only 2 of her chats (and these are typically single chats). The concentration of messages in few chats per user is shown in Fig. 7.2 (left), were we plot for some selected users (one curve per each user) the message 
distribution over the chats belonging to the user sorted by message volume. It illustrates the diversity across the users.
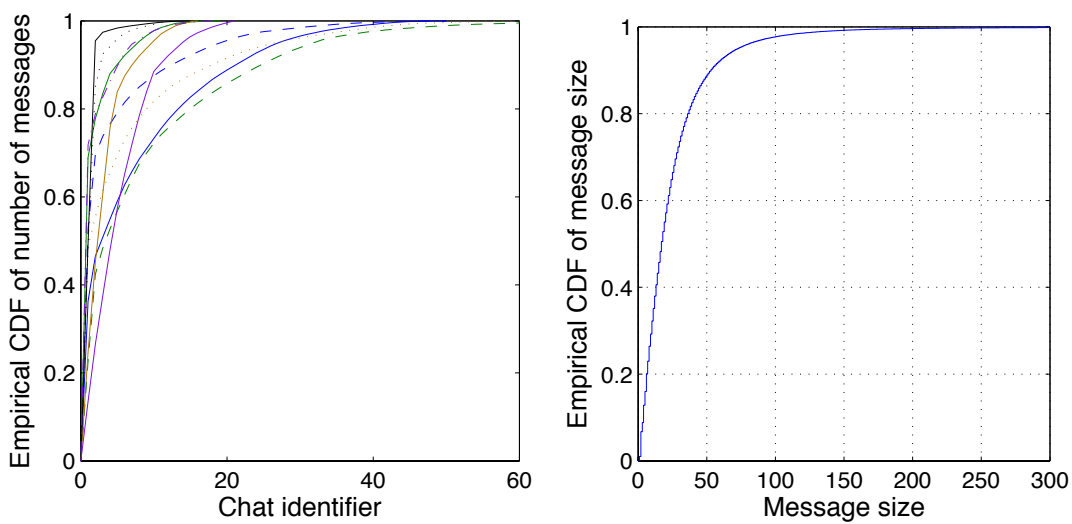

Figure 7.2: Message distribution of selected users over their chats (left) and overall message size distribution (right).

To summarise, the great diversity among users makes the usage depend much on the type of chat they employ. Differentiating these cases is interesting in order to employ different techniques to save energy. For example, some users might receive many messages from group chats while not using their device, leading to high energy consumption. A downlink message batching policy can reduce the energy consumption for this type of users.

\subsubsection{Message size}

Regarding message size, we observe that short messages are predominant. The average message size is 26 characters. Fig. 7.2 (right) shows that messages shorter than 40 characters comprise $83 \%$ of all the messages. Small messages lead to small packets, making it very inefficient to send each message in a separate packet. For example, if the transmission of these small messages is performed over TCP/IP (i.e., the most common case), the overhead created by the packet headers (40 bytes) is the same size as the payload (i.e., the message).

In order to understand which message sizes produce more traffic we multiply the message size by the number of messages. Fig. 7.3 shows the distribution of the total traffic over the message size and the proportion of the different origins (single/group chat and in/out). Most of the traffic is generated by the small packets in the case of IM.

Compression techniques can be used to replace recurring patterns in the data. Various works show the benefit of compression for transmitting less network data and thus reducing the energy cost [194, 255,262]. We analysed the compression performance of the default compression strategy 


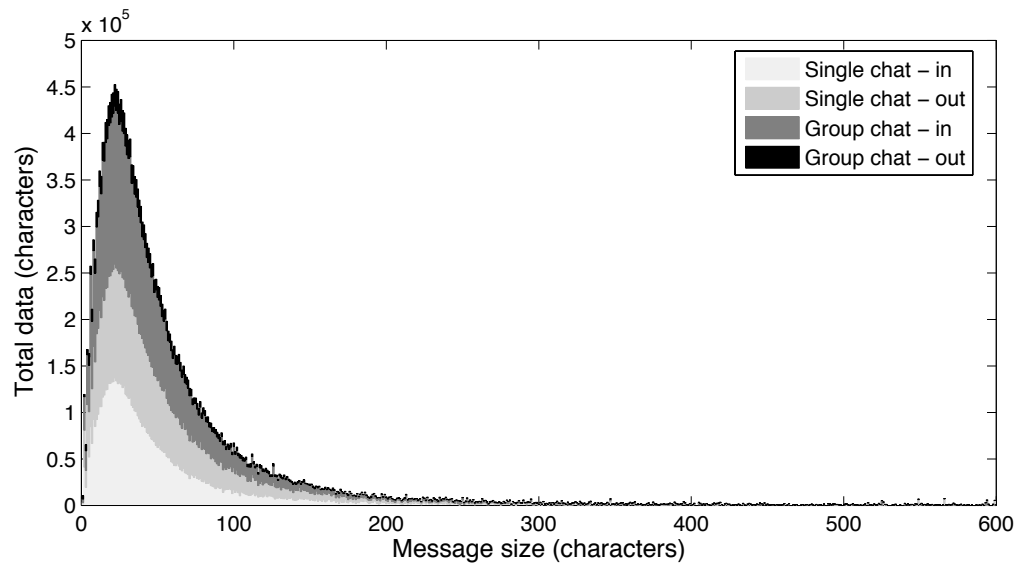

Figure 7.3: Distribution of the total data sent over the message size.

in Android $^{1}$ on the actual messages from the collected WhatsApp logs. Since most of the messages are short messages with few recurring patterns, we found that only $2.3 \%$ of the messages of our dataset would benefit from compression.

\subsubsection{Temporal properties}

Fig. 7.4 (top-left) shows the distribution of messages appearing in the collected dataset. As expected, the users tend to exchange more messages during the afternoon and the evening. Looking at the aggregates, the number of messages gradually grows during the day and peaks at 20:30 in the evening.

Fig. 7.4 (bottom) suggests that there is no clear weekly pattern in contrast to the cellular traffic observed in other works [225], where the daily peaks observed on the weekdays are higher than the ones observed during weekends. Every day of the week shows a similar trend and the number of messages does not significantly vary during the weekend.

Regarding the usage of every user, Fig. 7.5 shows the average and standard deviation of the number of messages sent per day and user. There is a great diversity across the dataset, from very active users with more than 200 messages per day to users that exchange few messages per day. The large standard deviation describes the variation for the same user between days, which makes it difficult to predict the IM traffic only based on the transmitted messages in the past.

The empirical CDF of inter-message interval (IMI) of the dataset is shown in Fig. 7.4 (top-right). We observe that messages with short IMI are predominant, where $73 \%$ of the messages have an IMI shorter than 1

\footnotetext{
${ }^{1}$ http://www.gzip.org/algorithm.txt
} 

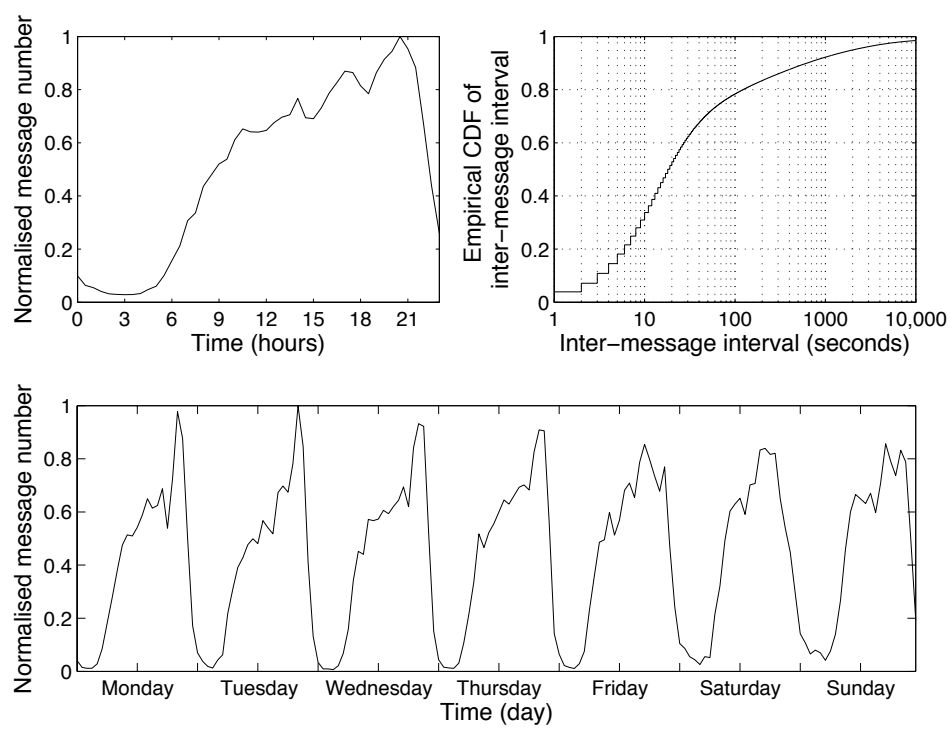

Figure 7.4: Normalised number of messages over hours in a day (top-left), empirical CDF of inter-message interval (top-right) and normalised number of messages during different days of a week (bottom).

minute. The long tail describes the distinct idle periods of the users.

We have observed that users generally write more than one message in a row before receiving any answer. This type of message is hereafter referred to as consecutive message and $48 \%$ of the messages in the dataset show this characteristic. From the energy perspective, consecutive messages are wasteful since they restart the inactivity timers of the cellular interfaces leading to higher energy consumption. If the elapsed time is greater than the inactivity timer, the UE consumes another energy tail. The short IMI of most messages, their small size and the significant presence of consecutive messages suggests that these could be coalesced to extend the idle time. In this paper we explore this option and present an algorithm that aggregates them in section 7.2.

Finally, two types of conversations (i.e., a chunk of the whole chat) can be identified over time. Based on the number of messages sent and their closeness in time, we can distinguish two different types of conversations over time: sparse and dense conversation periods. Sparse conversation periods have their messages more separated in time (i.e., higher IMI), while the dense ones represent intensive periods of interaction with shorter IMIs. This knowledge is used later on to select the evaluation traces.

To sum up, the dataset provides valuable insights for studying the message exchange characteristics of different users and efficiency of transmissions. 


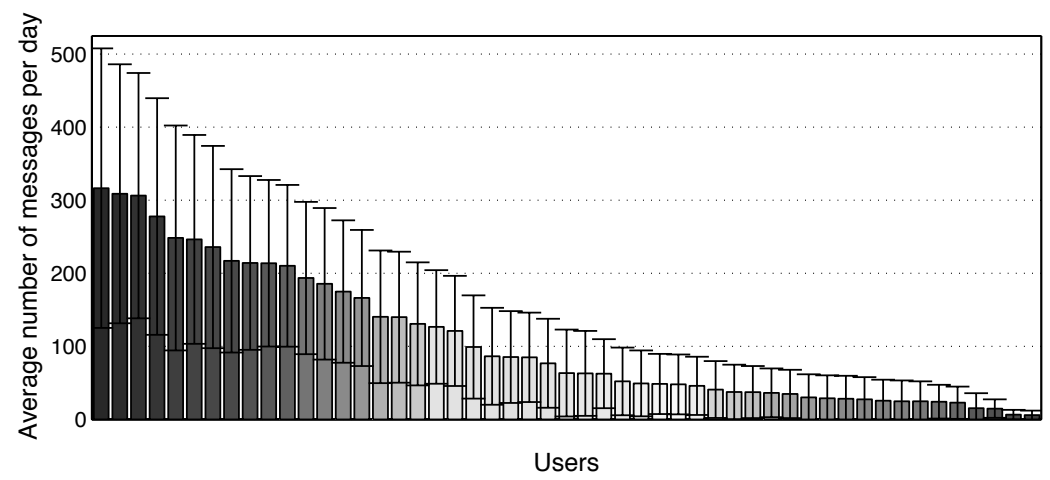

Figure 7.5: Average and standard deviation of messages per day per user.

\subsection{Bundling of messages}

Bundling aggregates consecutive messages by sending them together, ideally in the same packet. The potential benefits are the following:

- Less protocol overhead: Since the small messages predominate the IM text traffic, sending the messages in the same packet payload instead of sending them in separate packets would reduce the amount of protocol overhead.

- Extended idle state time: Reducing the number of transmissions by aggregating messages in the same bundle allows the UE to extend its idle state time, and therefore reduce the energy consumption.

- Opportunity for better compression: We conjecture that aggregating messages would improve the compression results. However, the impact of compression is not studied in this work.

Even though the benefits of message bundling are convincing, the main drawback is that the technique delays messages in order to send them together. Nevertheless, it is unclear whether introducing delay for consecutive messages is detrimental for the QoE when the other user is not active in the conversation.

In order to investigate the potential energy savings of message bundling and the impact on QoE in terms of message delay, we propose an event based algorithm, which uses user interaction information from the graphical user interface. Algorithm 5 describes the operation of message bundling. The intuition behind the algorithm is that when the user presses the send button the message is not directly sent over the network. Instead, a BundleTimer is started (or restarted if it is already running). The content of the text input area (i.e., the message $\mathrm{m}$ ) is queued in the message queue $Q_{M}$.

Whenever the text input area is changed, it means that the user is typing the next message. Therefore, the BundleTimer is restarted every time the 


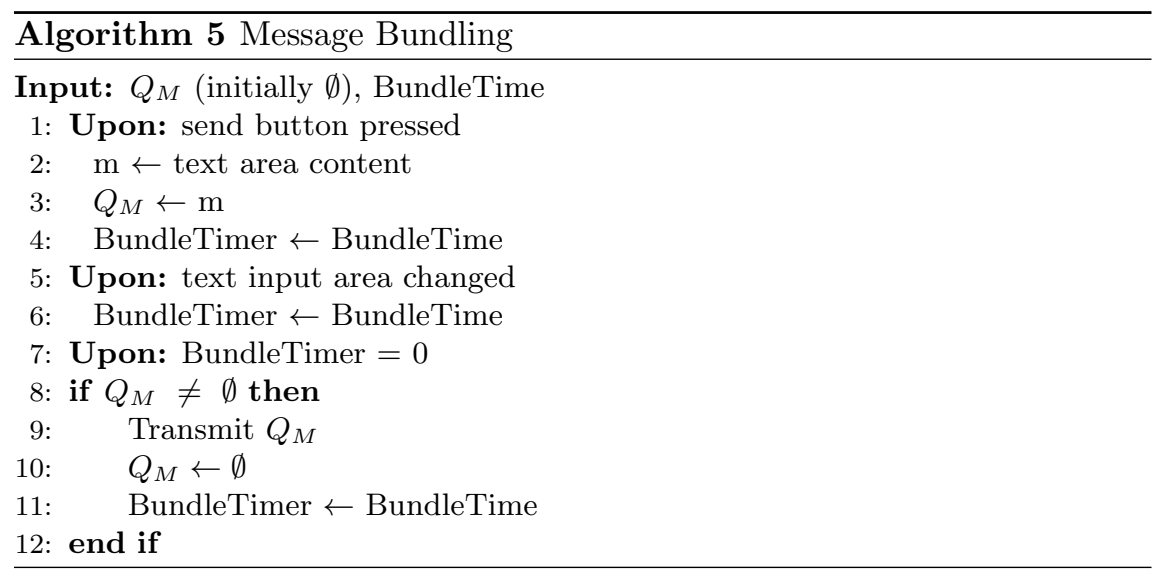

user changes the text, so that the new message can be bundled too. This prevents the $Q_{M}$ contents to be transmitted while the user is typing, thus recognises consecutive messages.

However, if the user never stops typing, the messages will never be sent. We argue that if the user keeps typing, the messages can be considered as the same one and QoE will not suffer from sending them together, no matter the length of the message. Therefore, we consider this case to be a pathological case.

The BundleTimer is a statically configured countdown timer, with the BundleTime as an input parameter. Whenever it expires, the messages in $Q_{M}$ are transmitted over the network, the $Q_{M}$ is emptied and the BundleTimer is restarted.

The proposed BundleTime parameter provides flexibility: a short BundleTime decreases the sending delay for single messages. A longer BundleTime would allow text over several consecutive messages to be aggregated. Since the "text input area changed" event requires the screen to be switched on, we do not consider more general indicators such as screen for simplicity.

\subsection{Typing notification}

The typing notification is a common feature implemented by most IM applications. The mechanism notifies the (receiving) user when the other (sending) user in the conversation is typing, creating a notion of presence and interactivity. Users can use this information to decide whether they should remain in the conversation or not.

In order to study the cost of this mechanism, we develop an algorithm which emulates its operation in a simple IM application. The notify updates should be triggered when the user types a character in the text input area on the graphical user interface. However, it is up to the implementation to decide the frequency of these events resulting in a network packet being 
sent to the receiving user as a notification. The typing notification feature increases the amount of packets transmitted, and potentially the energy consumption.

Algorithm 6 employs a countdown timer named NotifyTimer to restrict the rate of notify messages and thus prevent a packet flood. The NotifyTimer is statically configured with a NotifyTime value in seconds. When the user is typing, a text input area changed event is triggered. If the NotifyTimer is not running (i.e., NotifyTimer $=0$ ), the algorithm will send a notification packet to the network and start the NotifyTimer right after. The next time the user types, the NotifyTimer will avoid a new packet being sent to the network. Consequently, if the user is continuously typing the algorithm performs only a single notification packet transmission every NotifyTime.

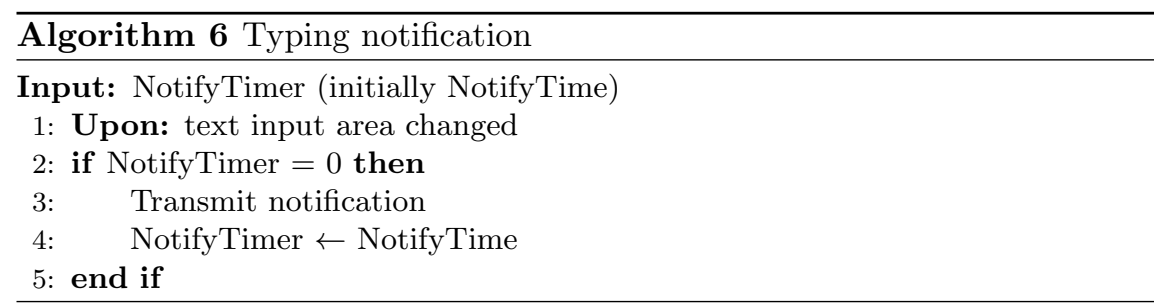

When the other user receives the typing notification, the receiving end of the application will show the receiving user the notification. For example, we have observed that WhatsApp uses approximately a 3 seconds timer for the NotifyTime.

\subsection{Evaluation methodology and settings}

This section describes the methodology and the evaluation environment used to quantify energy consumption of the typing notification and message bundling.

Similarly to previous chapters, the general methodology is as follows: a predefined set of real conversations is automatically replayed between a pair of clients using a prototype IM application running on commodity devices. The conversations are transformed to network transmissions (i.e., packet traces) by our prototype application. The communication is performed through a real $3 \mathrm{G}$ network and the resulting packet traces are captured. The energy is calculated using EnergyBox from the captured real packet traces.

First, the energy consumption of each conversation is calculated as a baseline (without typing notification nor message bundling). Second, the conversations are replayed with only message bundling enabled (Algorithm 5 ), and the energy savings are calculated and compared against the baseline. Third, the conversations are replayed with only the typing notification 
enabled (Algorithm 6), and the extra energy cost is calculated by comparing the resulting energy consumption against the baseline.

First, we briefly describe the evaluation environment as well as the evaluation conversations and EnergyBox.

\subsubsection{IM application}

The client application provides the chat functionality between two Android devices and implements the logic of Algorithms 5 and 6. The implementation of the message bundling and the typing notification feature are based on instances of the Async Task class provided by Android, where the Async Task represents the timers that can be cancelled, reset and started again.

We selected the Message Queueing Telemetry Transport (MQTT) protocol as the transport protocol for the following reasons: it is a lightweight application protocol, some IM applications officially use it (e.g., Facebook Messenger), and the Mosquitto open source project provides easy to set up public MQTT servers that accelerate the development phase. MQTT is a publish/subscribe protocol, where the subscribers are instantaneously notified whenever a publisher generates a new event.

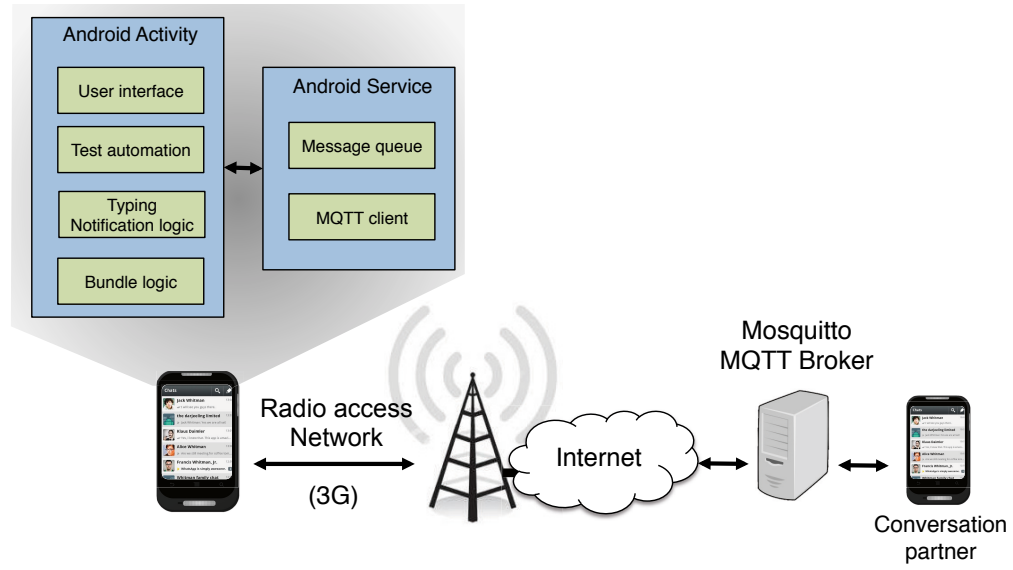

Figure 7.6: Architecture of the IM prototype implementation and the test environment.

Fig. 7.6 shows the general architecture of the application and the test environment. The IM clients are able to communicate using MQTT through the server that hosts the MQTT broker. Each user subscribes to her nickname. The conversation partners send a message by publishing the message to the nickname (in publish/subscribe terms, the topic) of the recipient. The MQTT broker keeps track of the subscriptions to different topics.

Since the IM applications tested in section 2.1 in the background chapter are black boxes, we cannot compare the results obtained from our prototype

\footnotetext{
${ }^{1}$ Mosquitto test server: http://test.mosquitto.org/
} 
application against them in a fair way. However, for the interested reader, the basic energy cost of the prototype application for the same conversation as that shown in section 2.1 is lower than the rest of IM applications (18 joules). More information can be found in Appendix C.

\subsubsection{Test traces and parameter settings}

This section describes the parameter settings and the conversations used to study the energy characteristics of the typing notification and the message bundling.

Test conversations: Four different test conversations are selected for the tests based on the different patterns observed in section 7.1. Two conversations (Dense and Sparse) were randomly selected from the dataset, representing intensive conversations and slow-paced ones characterised by short IMIs and longer IMIs respectively.

Random is a synthetic conversation generated by randomly selecting messages from the dataset not considering the resulting conversation's dense or sparse characteristics. By randomly selecting the messages we aim at obtaining messages of different sizes. The selection follows the following rules. Message pairs in the resulting conversation have an IMI of shorter than 30 s since larger IMIs are uninteresting from the energy perspective (greater than the typical inactivity timers). Moreover, if a selected message appears in a sequence of consecutive messages, then the other messages belonging to that sequence are also selected to keep the realistic consecutive message relations from the dataset.

Finally, Short is the same conversation used in the background chapter (section 2.1) to compare the different IM applications. It characterises a trace containing short messages, a single consecutive message, and IMIs between the Sparse and Dense conversations. The four categories have different number of consecutive messages, which is interesting in order to test the message bundling.

The test conversations used are shown in Fig. 7.7. The duration of the selected conversations is below $250 \mathrm{~s}$. We believe these are representative conversations for IM. Since our dataset did not delimit conversations (the users were always logged in and no distinction of different conversations were made within the chats), we base our reasoning about duration on earlier work [265], where 9900 of approximately 10000 conversations were shorter than $250 \mathrm{~s}$. Note that in Fig. 7.7 the conversations do not start from 0 since the time to write the message is also considered, and the difference in their length is not important since the results are studied per conversation.

Writing speed parameters: In order simulate the user typing and conveniently automate the tests, the prototype application is instrumented with the test automation capability that simulates the user writing characters at a parametrised writing speed. For each message of a given conversation, the 

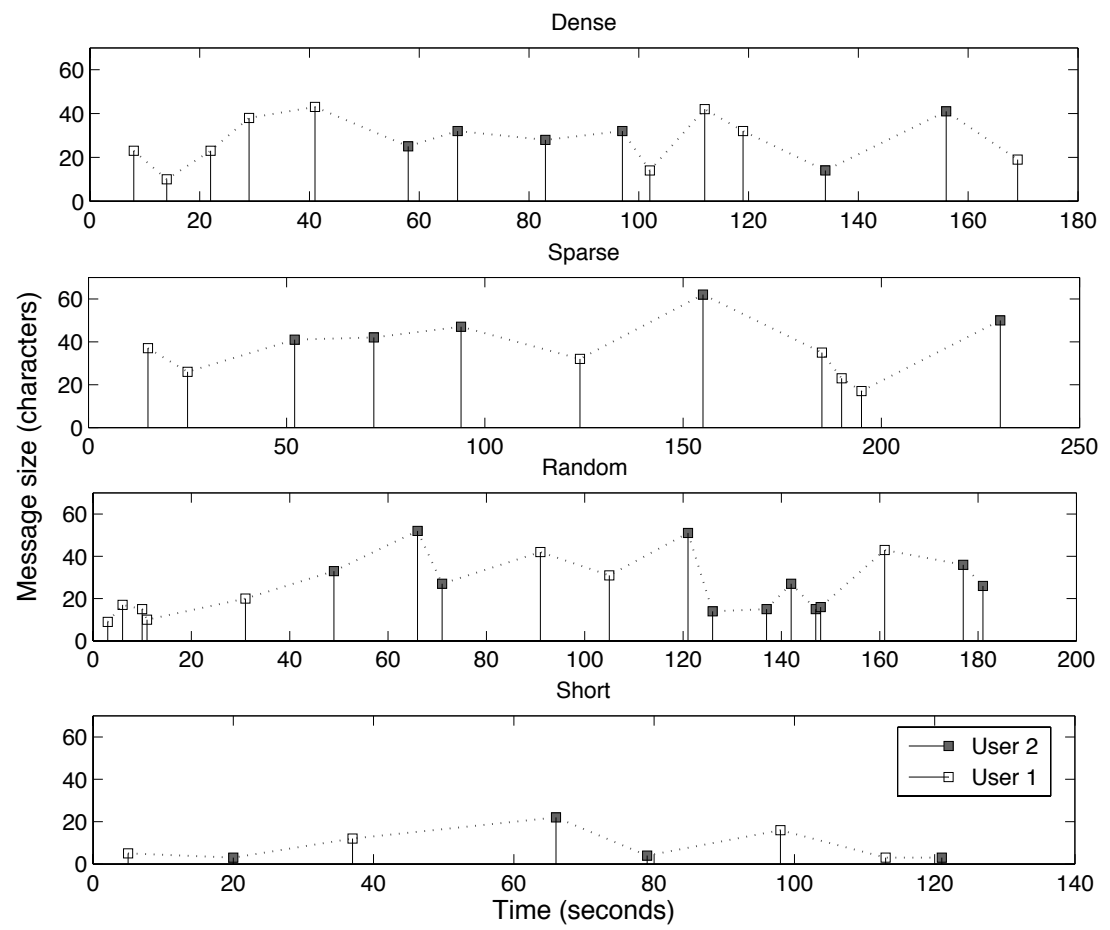

Figure 7.7: Test conversations.

characters of the message are written using a constant typing speed. The typing automation logic starts writing in the input text field of the application so that the message is sent in its correct timestamp. The time to start writing the message is calculated using the message length and the average writing speed.

For simplicity, the typing speed is set to a constant of $287 \mathrm{~ms}$ delay per character. This number was experimentally obtained by averaging the measured time to write 160 characters (i.e., a SMS) for 6 different people.

Communication energy: While replaying the sequence of messages of the test conversations, the packet traces are captured in the device of the user 1 using tcpdump for every test. Only the traffic of the IM application is allowed using a firewall to block the rest of the traffic. The communication energy is computed from the traces using EnergyBox with the parameters shown in Table 3.1. The UE power values for the different RRC states are based on earlier measurements [198]: $P_{D C H}=612 \mathrm{~mW}, P_{F A C H}=416 \mathrm{~mW}$. We set $P_{P C H}=0 \mathrm{~W}$ to quantify only the energy spent for data transmission.

The evaluation methodology and environment are employed to analyse the potential energy savings of the message bundling and the cost of the typing notification in the next sections. 


\subsection{Does message bundling pay off?}

Next, we quantify the energy savings that message bundling can provide at the cost of an introduced delay due to aggregating messages.

\subsubsection{Energy savings and bundling results}

Each test conversation is tested with the following BundleTimes: 1, 3, 5 and $7 \mathrm{~s}$. The results are normalised to the base energy consumption with the message bundling disabled $(32.63,26.68,32.81$ and 17.81 joules for Dense, Sparse, Random and Short respectively). The results are based on 3 repetitions of each unique test. Additional repetitions are run when large standard deviation is observed.
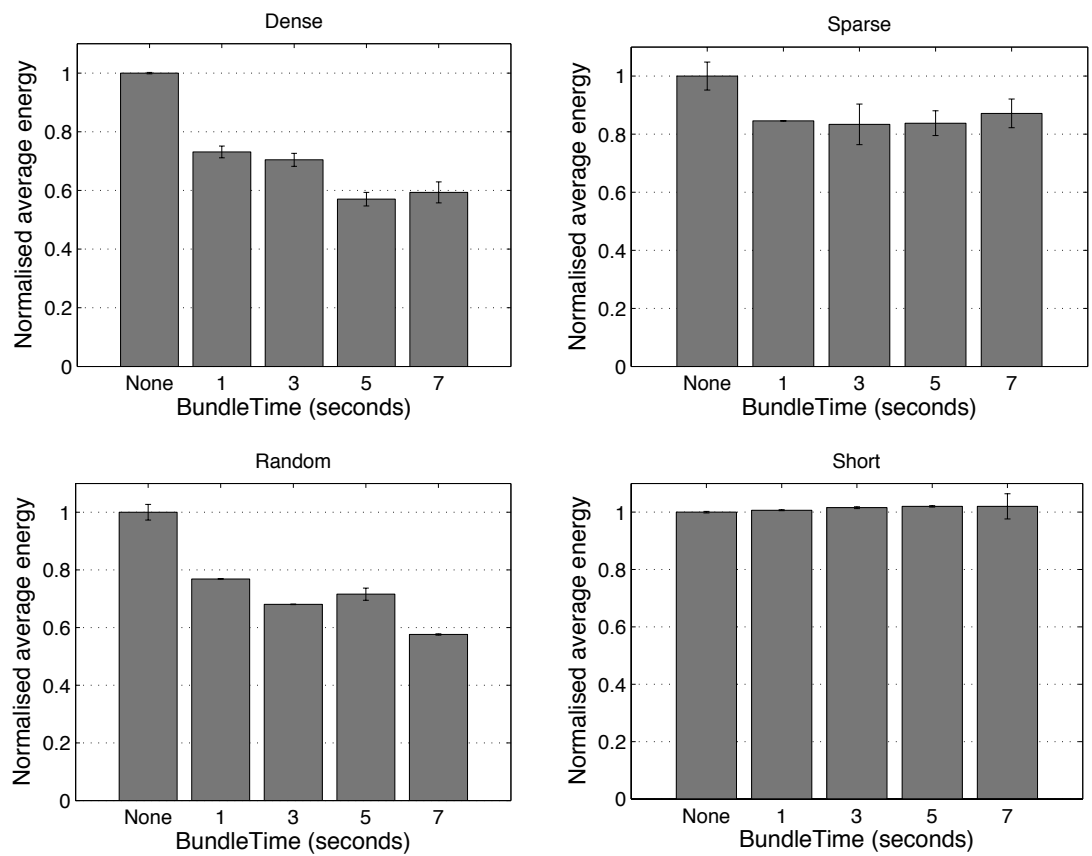

Figure 7.8: Normalised average energy and standard deviation for the effect of message bundling for the different conversations.

Energy savings: Fig. 7.8 shows the energy savings for the different test conversations. The message bundling provides energy savings when the algorithm successfully performs at least one bundle. The energy savings vary between the conversations, and thus we describe them separately.

The energy savings due to bundling range from $27 \%$ to $43 \%$ for the Dense test conversation. Since it contains many consecutive messages and these 
are often close in time (i.e., the user starts writing soon after a previous message is sent), even a short BundleTime of $1 \mathrm{~s}$ can achieve $27 \%$ savings.

The results for Sparse are different. The message bundling achieves $16 \%$ energy savings with a BundleTime of $1 \mathrm{~s}$. However, increasing the timer value does not increase the energy savings. The IMI of the consecutive messages is long for the Sparse conversation, thus the short BundleTime values do not allow performing most of the possible bundles.

Regarding the Random conversation, the energy savings range from $24 \%$ to $42 \%$ for the different BundleTimes. The shortest timer provides again significant energy savings.

There are only two consecutive messages in the Short conversation. No bundle is created since the inter-message interval of the two consecutive messages is longer than the BundleTimes, and the message lengths are short (i.e., the typing time is short).

Bundling results: Tables 7.2 and 7.3 show the achieved number of bundles and the messages per bundle for the different BundleTimes using the Dense and Sparse conversations. Possible bundles refers to the number of distinct bundles where each bundle is a sequence of consecutive messages with the maximum length appearing in the conversation.

For Dense, the BundleTime of $1 \mathrm{~s}$ creates 3 bundles out of the 4 possible bundles (i.e., 4 groups of consecutive messages). Only one more message is aggregated for the $3 \mathrm{~s}$ BundleTime. All the possible bundles are performed when the BundleTime is increased to $5 \mathrm{~s}$ achieving the maximum energy savings. Similar results are obtained for 5 and 7 s, i.e., no additional messages are bundled even increasing the BundleTime.

Table 7.2: Number of bundles and bundles per message for the Dense conversation.

\begin{tabular}{|c|c|c|}
\hline BundleTime (s) & Bundles & Messages per bundle \\
\hline 1 & 3 & $3,3,2$ \\
3 & 3 & $4,3,2$ \\
5 & 4 & $5,3,3,2$ \\
7 & 4 & $5,3,3,2$ \\
\hline \hline Possible bundles & 4 & $5,4,3,2$ \\
\hline
\end{tabular}

For Sparse, the BundleTime of $1 \mathrm{~s}$ leads to a single bundle out of the 3 possible bundles. The 3,5 and 7s BundleTimes only create an additional bundle of 2 messages.

Protocol overhead: The message bundling also provides less TCP/IP header overhead. The number of packets sent in the Random conversation with the message bundling disabled were reduced from 40 to 16 when using a BundleTime of $7 \mathrm{~s}$. Thus, the messages are sent in the same packet reducing the TCP/IP header overhead. 
Table 7.3: Number of bundles and bundles per message for the Sparse conversation.

\begin{tabular}{|c|c|c|}
\hline BundleTime (s) & Bundles & Messages per bundle \\
\hline 1 & 1 & 3 \\
3 & 2 & 2,3 \\
5 & 2 & 2,3 \\
7 & 2 & 2,3 \\
\hline \hline Possible bundles & 3 & $2,3,3$ \\
\hline
\end{tabular}

To sum up, our results show that even a short BundleTime can lead to significant energy savings. The next section studies the potential drawback of message bundling.

\subsubsection{Message delay}

Even though the bundle technique is desirable from the energy perspective, one needs to also consider its negative impact on per-message delay. The bundle technique delays each message by a minimum of the BundleTime value. The delay of the held messages increases when the user continues typing a consecutive message since the BundleTimer is restarted every time the user types a character.

The per-message delay is computed for the Dense and Sparse test conversations for different BundleTimes. The application is instrumented in order to obtain the delay between the moment of pressing the sending button and the time that the message is actually sent to the network. The minimum delay for each BundleTime is the BundleTime itself, representing the case that a message was not bundled. The bundled messages are typically delayed more than the minimum, depending on the IMI, the BundleTime and the number of characters in the next message.

Fig. 7.9 shows the delay introduced by bundling in the Dense and Sparse conversations. The median delay for the Dense conversation with the BundleTimes 1 and $3 \mathrm{~s}$ is the BundleTime since most messages are just delayed by the minimum delay. These are the last messages added to a bundle or the messages that were not bundled. As expected, the bundled messages experience greater delay.

Fig. 7.9 shows that the maximum delay for the BundleTimes 1 and $3 \mathrm{~s}$ is 20 and 30 seconds in the Dense scenario. The maximum delay for $3 \mathrm{~s}$ BundleTime increases because it bundles an additional message. The more messages in the bundle, the higher is the maximum delay of the first bundled message. However, increasing the BundleTime leads to a great increase of per-message delay, especially for the messages that are bundled. In Dense, even though the BundleTimes 5 and $7 \mathrm{~s}$ form exactly the same bundles, the messages experience an extra delay with no extra energy saving.

The results for the Sparse conversation show that the median delay is 

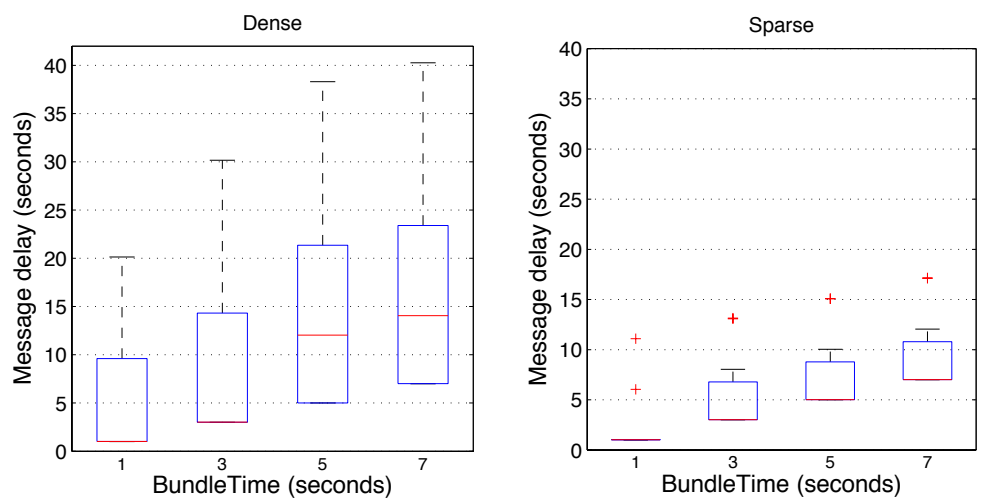

Figure 7.9: Box plot of the delay experienced by the different messages for Sparse and Dense conversations.

the minimum experienced delay. The long IMI of the Sparse conversation make the bundle technique to create a single bundle of 3 messages for the $1 \mathrm{~s}$ BundleTime (the outliers of 6 and $11 \mathrm{~s}$ in Fig. 7.9p, and an additional bundle of 2 messages for the rest. Thus, the per-message delay is shorter due to the smaller number of bundles than for the Dense conversation.

Comparing the energy savings and the introduced per-message delay, we observe that a short BundleTime of $1 \mathrm{~s}$ leads to significant energy savings while not causing huge delays. This indicates that keeping track of the user typing is enough for a simple bundle policy. Longer BundleTime values can increase the energy savings for dense conversations at the cost of higher delay. However, Sparse conversations with sporadic messages should not use long timers.

Finally, the message delay does not always have a negative impact on the QoE. When the user is not engaged in an active conversation, the reception of the non-delayed messages or a bundle of delayed messages can be argued to be the same. However, from the energy perspective, the latter drastically reduces the energy consumption.

\subsection{Cost of typing notification}

Aggressive notification policies can lead to energy waste. This section studies the additional energy cost incurred by the typing notification functionality for the different test conversations. The results are based on 3 repetitions of each unique test. An additional 2 repetitions are run if large standard deviation is observed in the results.

For each conversation, we compute the energy consumption with the typing notification functionality disabled as a baseline. We select 3,5 and $10 \mathrm{~s}$ as NotifyTime values for the different tests. These values are representative of the parameters used in real typing notification mechanisms. 
Since the focus is on the additional cost, we normalise all the values to the average energy consumed by the baseline for each conversation (30.65, 26.99, 33.36 and 18.47 joules for the Dense, Sparse, Random and Short respectively), i.e., the energy cost with no typing notification.
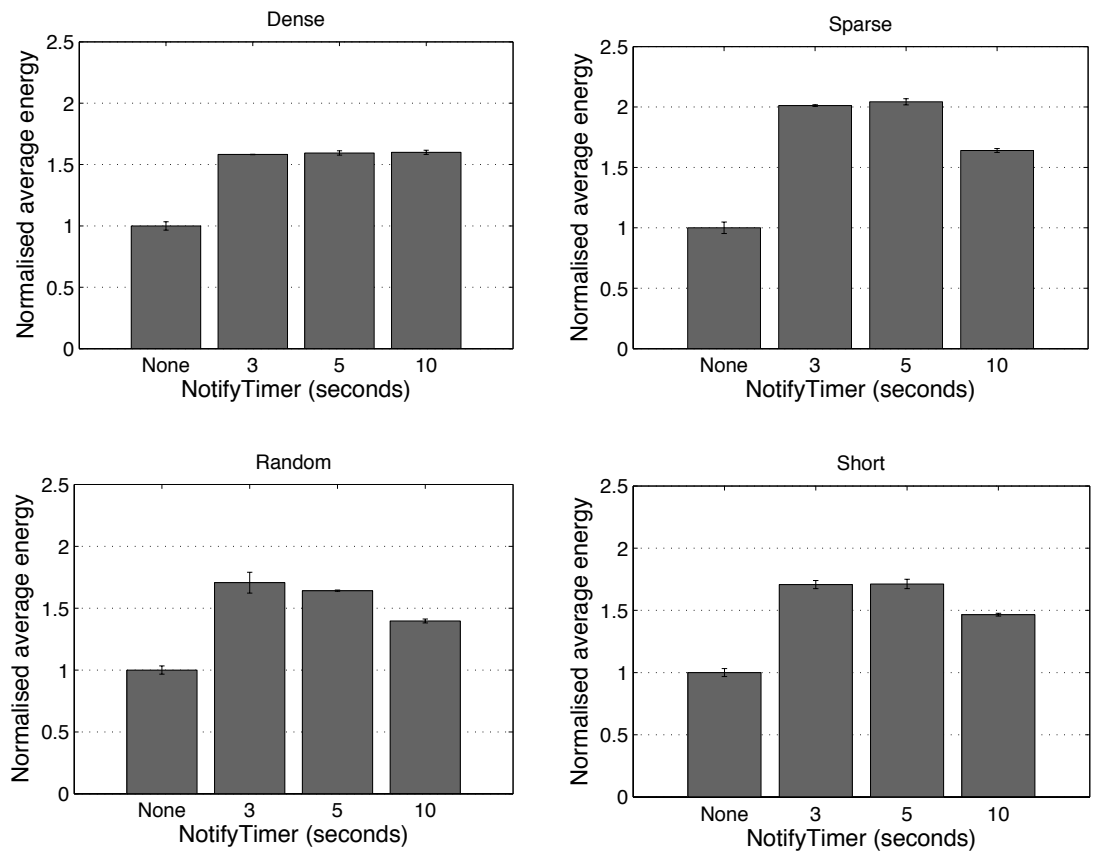

Figure 7.10: Normalised average energy and standard deviation of the typing notification feature for the different conversations.

Fig. 7.10 shows that in general the additional energy cost of the typing notification feature is large, varying from 1.4 to 2 times the base energy consumption (40-104\% more energy). Since the functionality sends packets whenever the user is typing, it keeps the $3 \mathrm{G}$ interface in an active state for almost the whole duration of the test. The results for each conversation are explained next.

For the Dense conversation, the energy consumption for the different NotifyTime values is similar. Even though a longer NotifyTime implies sending fewer notify packets, when the device enters a high energy RRC state, sending more data does not incur a higher energy cost. Most of the energy consumption is due to reseting the inactivity timers by the operator, and thus the message pattern of the conversation greatly influences the consumed energy. Since the conversation is dense, the impact of the typing notification is not as high as in the Sparse conversation.

The additional energy cost for the Sparse conversation is higher than the Dense one. The Sparse conversation has periods of time where the de- 
vice is in $\mathrm{PCH}$ between message sendings. However, the typing notification messages greatly reduce the idle time. Since the 3 and $5 \mathrm{~s}$ NotifyTimes are shorter than the inactivity timer $T_{2}$, the $T_{2}$ timer is reset before it has expired. When the NotifyTime is $10 \mathrm{~s}, T_{2}$ expires, which results in higher idle time. However, the energy cost is still $64 \%$ more than the baseline. The Sparse conversation is characterised by having slightly longer messages, and thus the time to type for the user is longer and more notify packets per message are sent.

The results for the different NotifyTimes for the Random and Short are similar to the Sparse conversation. The Short and Random conversations contain mostly short messages. When NotifyTime is $10 \mathrm{~s}$, less notify messages are sent since the user spends less time typing the messages.

To sum up, the typing notification functionality results in a high additional cost for an IM application. Even though a longer NotifyTime avoids excessive usage of the $3 \mathrm{G}$ interface, the cost is still high.

\section{7 $\quad$ Summary and discussion}

When developing energy-efficient solutions for application data communication, there is a trade-off between new application functionalities and energy conservation. There is a need to quantify the extra energy cost and the perceived functionality benefit from the user side. EnergyBox is a valuable instrument for this purpose.

In this chapter we quantified the typing notification functionality employed by most IM applications and showed its tremendous energy cost. According to our results, this functionality can increase the energy consumption of an IM application by $40-104 \%$ from the basic message exchange functionality. Quantifying the cost of a functionality can allow the developers to rethink the need for the functionality or provide the option to dynamically enable/disable it.

When the traffic is directly generated by user interaction, the network transmissions can easily result in energy waste. In our work we collected and studied an IM dataset from mobile users to create a better understanding of the user inputs that trigger network communication. Based on our study with collected real usage data, we observe that IM application users currently tend to write consecutive messages, which inefficiently increases the active time of the wireless interface. Thus, we believe that bundling can be used to lower the number of transmissions while the user continues typing, and send them at once. The solution matches users' common utilisation to the energy characteristics of cellular networks by combining multiple transmissions in a single energy event at the cost of additional delay.

We show that informed by user interaction (e.g., GUI inputs) bundling can be used to reduce transmissions while the user continues typing, and send them at one go. Our results show energy savings up to $43 \%$ depending on the message pattern of the conversation. Given the high percentage of 
the consecutive messages, this is a promising result. However, using longer timers for the bundle technique can lead to high delays for some messages. Thus, the bundle technique can be dynamically activated for sparse conversations, or when the receiving end of a chat is offline or away. This could be automatically deactivated in periods of high intensity conversations, i.e., for real-time communication with high responsiveness requirements.

More sophisticated bundling techniques are feasible leveraging knowledge of user activity (e.g., the screen is off, the user switched the focus to another application or historical data), message content parsing (e.g., conversational closings such as "talk to you later"), context information (e.g., built-in sensors such as the accelerometer $[15,115]$ ) or presence information from other users. Distinguishing between periods of sparse and dense conversations is interesting to dynamically activate the bundle technique (e.g., moderately using the typing notification). Considering multimedia messages is also a future direction.

To sum up, our work shows the feasibility of tailor-made solutions for specific application types to lower their energy consumption. Conceptually, the proposed solutions provide a trade-off between energy and performance (i.e., QoS parameters such as delay) and are applicable to other contexts as well. A more detailed knowledge of the specific mechanisms of applications or usage characteristics can inform more appropriate tailor-made energyefficient solutions.

However, the energy contribution of an application to the total system is not only determined by its isolated energy $E(\{i\})$. The interaction of the other applications can drastically impact the total energy consumption $E(N)$. The next chapter studies general methods to determine the energy contribution of different entities to a system's $E(N)$ and the potential implications of selecting a method. 
"The world isn't fair, Calvin."

"I know Dad, but why isn't it

ever unfair in my favor?"

Bill Watterson

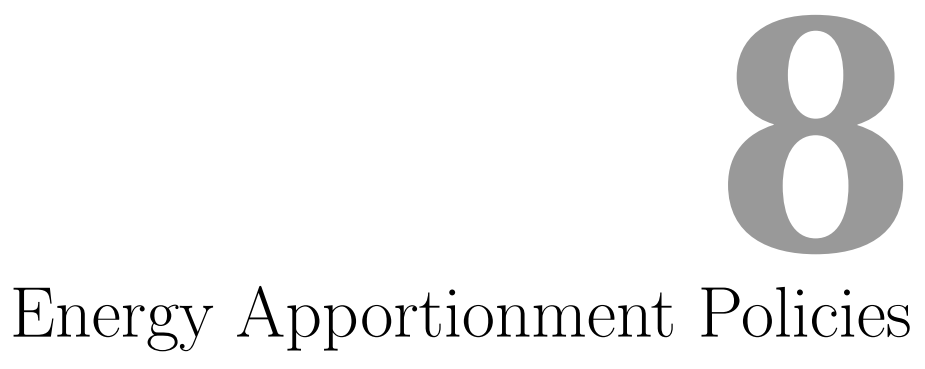

In previous chapters we provided means to estimate the energy consumption of the system $(E)$ as well as reduce the energy consumption of a given application isolation $(E(\{i\}))$ and the energy consumption of a group applications $(E(S))$ in the mobile communication context. However, we neglected the impact of the interactions among the different entities and the resources in the system (i.e., applications and the wireless interface in our context). This impact is not negligible as every entity contributes to the total energy consumption $E(N)$, and the interactions and coexisting accesses often result in $E(N)$ not being the sum of what the entities would consume using the resource alone. In this chapter we broaden the perspective and look at all the entities in a system to provide methods to support energy management, which are also applicable to our context in mobile communication. In particular we are interested in determining the contribution of an entity to the total energy consumption of the system.

Energy accounting is the procedure of quantifying, analysing and reporting the energy consumption of different entities or activities of interest in the system. Efficiently managing energy requires a detailed understanding of the level of resource consumption and energy accounting is a key medium to provide the needed transparency to the system. Energy accounting is also vital to evaluate the energy efficiency of entities in the system. There is evidence supporting that energy awareness can aid to conserve energy [20,184]. Unfortunately, apportionment is a hard problem of accounting in general which does not have a single best solution for all cases [129], and energy accounting does not escape from this problem.

Selecting an apportionment method, i.e., a method to divide the shared 
energy consumption, entails a set of principles used to achieve some rational division. Thus, we call these methods energy apportionment policies referring to the rule that prescribes the consumed energy to each entity. Examples of entities are different applications using a certain resource, processes of interest or system users. We illustrate the apportionment problem with two instances in the context of mobile and wireless networks, but as we show later in this chapter the problem arises in several other contexts.

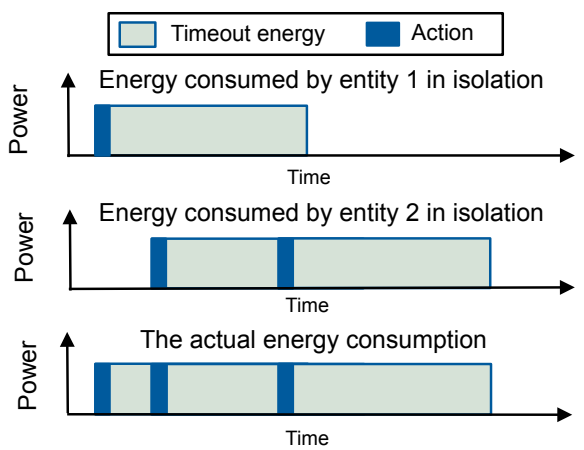

System states:

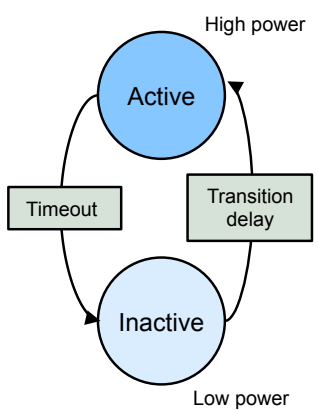

Figure 8.1: Energy apportionment example for a simplification of a common system.

Fig. 8.1 exemplifies a simple apportionment case. The system is characterised by two states, active and inactive, where an entity can perform an activity only in the active state. This is shown in Fig. 8.1 (right). After every activity a timeout is activated which keeps the system consuming energy in the active state for a while. The timeout is a power management mechanism that also serves responsiveness by avoiding the inactive to active state transition delay for subsequent activities. This is the notion behind the inactivity timers and the tail energy discussed in previous chapters. Many computing and communication components or systems show a similar behaviour, such as hard drives [172], Secure Digital cards [173], the already discussed wireless interfaces $[24,152,173]$ and even some electric appliances [207].

Fig. 8.1 (left) shows the energy consumption of the entities if they were alone in the system (i.e., in isolation). The actual total consumption of the interface is not the sum of entities' consumption in isolation. How should we prescribe a share of the total energy consumption to the different entities in the system? Equally dividing the energy consumption would mean that entity 1 subsidises entity 2 . Would a proportional apportionment policy be better?

Another example is from a wireless sensor network scenario (also applicable to an Internet of Things context) [83,116], where determining the energy consumption of network nodes due to user defined activities (e.g., queries) is required. In this scenario, the total energy consumption of the system is determined by the sum of each node's consumption, but the contribution of 
each node to the total consumption is not necessarily its own consumption. A node contributes to other nodes' energy consumption by being part of the system (e.g., transmissions or beacons). Determining the contribution of each node and the consumption due to the user-defined activities requires a solution to the apportionment problem as well.

Selecting an apportionment policy is a fundamental choice for a system, not only it enables the assessment of the energy consumption of an entity in the system, but we show that it also establishes the "rules of the game" for the system which potentially influence the behaviour of the entities, e.g., by providing incentives. The latter aspect is related to fairness of the apportionment that is often hidden at first sight in an energy apportionment policy. Tijs et al. [238] affirm that the choice of a solution for cost allocation depends on many factors such as the concrete situation or system, ideas of the designers or participants respect to fairness and the difficulty of calculating the cost allocation.

In our work we show that energy apportionment is a common problem in different areas of computer science, ranging over computing [79, 217], data centres [31], mobile devices [63, 152,161,173,212,278], wireless sensor networks [116,230], and energy-efficient buildings [46,97,236,243]. Our work is the first recognising the common ground of the energy apportionment problem in these areas and builds a bridge to study the this problem through the lens of cooperative game theory. This provides excellent mathematical methods to allocate cooperative costs and evaluate the solutions already used in multiple real world practical problems $[78,156]$. Additionally, in this chapter we:

1. Identify 5 relevant fairness criteria for energy apportionment. We show that providing incentives to improve the energy efficiency of the system, neglected in previous works, is a relevant purpose of an energy apportionment policy.

2. Recognise 8 different energy apportionment policies already proposed in different fields to provide a common understanding of the energy apportionment problem. In addition, our work formalises 3 of them not previously presented mathematically.

3. Propose 2 alternative novel policies based on cooperative game theory not previously used in energy apportionment. These policies provide a strong fairness notion and a rich incentive structure.

4. Analyse the information requirement and computational complexity of the 10 energy apportionment policies.

5. Evaluate the fairness of the 10 policies employing the identified 5 fairness properties.

Our analysis shows that there is a trade-off between fairness and the other evaluation criteria, and provides guidelines to select an policy de- 
pending on the purpose of the energy apportionment and the characteristics of the system. In particular, for the 5 formal fairness properties we show that the proposed game theoretical policies provide the strongest fairness. However, these policies have a high information requirement and computational complexity, and thus other simpler policies are identified to provide a satisfactory compromise when the fairest policies become unpractical. We also classify the policies based on the type of input information used, and pinpoint that if surrogate information (e.g., usage time) is used in the apportionment, this has to correlate with the energy consumption of the system in order to provide correct incentives to reduce the energy consumption.

The rest of the chapter is structured as follows: Section 8.1 introduces our system model as well as the background, and section 8.2 describes the 10 energy apportionment policies in their application context. Section 8.3 compares the policies in terms of required information and computational complexity. In section 8.4 we leverage game theory to provide a formal study of the fairness characteristics of the different energy apportionment policies. Section 8.5 summarises and discusses the insights of our analysis. Finally, section 8.6 summarises the chapter and discusses the results. We briefly comment the results considering the context of the thesis.

\subsection{Notation and background}

In order to formulate the different energy apportionment policies we first introduce our system model, a time model employed to formalise some policies and the corresponding notation used in the rest of the chapter. We also introduce the energy apportionment policy definition and the required background from cooperative game theory to understand our analysis.

\subsubsection{System model}

An energy consuming system is composed by a set of entities $N=\{1,2, \ldots, n\}$ that cause the system which they are part of to consume energy. We distinguish two types of system interpretations: (1) common resource, where the entities share a common energy resource, and (2) individual resource, where each entity has an energy resource. These system interpretations were briefly introduced in Chapter 1 . We now restate some of the notions used in the thesis in a formal manner.

Examples of a common resource system are the computers in a datacenter or a battery-powered device shared by different applications, whereas a set of mobile devices or sensor nodes in an ad-hoc or sensor network are systems with individual resources. 


\section{Common resource system}

$E(N)$ is the total energy consumption for a system configuration of the $N$ entities sharing a common resource. We assume that equipment to measure $E(N)$ is available, e.g., measuring the power consumption of a phone by intercepting the battery terminals (similar to our setup presented in the Appendix B) or the mains connected to a home appliance [46].

$E(S)$ is the isolated energy consumption of the entities in $S \subset N$. Thus, $E(\{i\})$ is the isolated energy consumption of the single entity $i \in N . E(\{i\})$ represents the energy consumption of the entity $i$ in the hypothetical case that it was alone in the system, not considering the interaction of the other entities in the system. This quantity is a construct which is estimated (generally not measurable), for example a single application running alone on a mobile device or computer.

\section{Individual resource system}

$E_{i}(N)$ is the individual energy consumption of the entity $i \in N$ given a system configuration of entities $N$. We assume that equipment to measure $E_{i}(N)$ at each entity $i$ is available. The total system consumption is $\sum_{i \in N} E_{i}(N)$, e.g., the total consumption of a network of sensor nodes is the sum of nodes' consumption in a network configuration ${ }^{1}$.

$E_{i}(S): i \in S, S \subset N$, is the individual energy consumption of the entity $i$ given a system configuration of entities $S$. This is a construct which is estimated (generally not measurable). The total energy consumption for a given $S \subset N$ is $\sum_{i \in S} E_{i}(S)$.

\section{Discrete-event time model}

The formalisation of some policies require to model time and thus we employ a discrete-event formal framework introduced in the context of real-time systems [127]. This section provides an overview of the model.

Atomic events occur along a physical time line where every event $e=$ $(t, v)$ is defined by a time point $t \in \mathbb{R}$ and a value $v \in V$. The value $v$ is a flexible representation specific to the system.

A discrete-event signal $s$ is a set of events and it cannot have two identical events. $D$ is the set of all all signals $(s \in D)$. The definition of a functional signal [127] states that the events in any $s$ can be enumerated chronologically, there is a single event per $t$ and that between any two time points there is a finite number of events.

Tuples of signals are used for naming purposes. A tuple $\mathbf{s}$ of $N$ signals is defined as $\mathbf{s}=\left[s_{1}, \ldots, s_{N}\right]$ where the set of all such tuples is $D^{N}$.

Fig. 8.2 shows an example of two discrete signals $s_{1}$ and $s_{2}$ with two value types $\left(v_{1}\right.$ and $\left.v_{2}\right)$ which are part of a discrete tuple $\mathbf{s}$.

\footnotetext{
${ }^{1} E(N)$ is only applicable to the common resource case.
} 


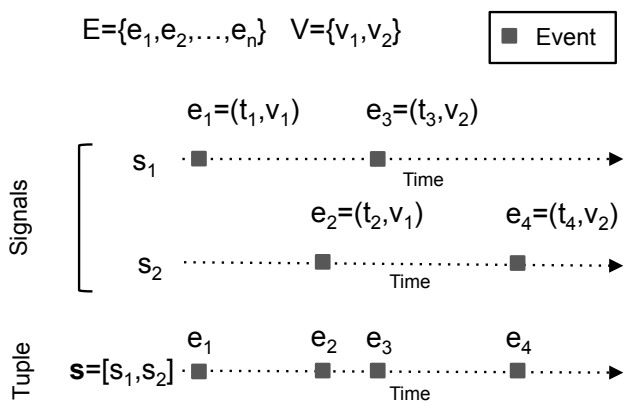

Figure 8.2: Example of the time model for two discrete signals and a tuple.

\subsubsection{Energy apportionment policy}

An energy apportionment policy $\Pi$ prescribes the share $\pi_{i} \in \mathbb{R}$ to an entity $i \in N$ of the total system consumption of the system composed by $N$ entities. A policy may use different input information to apportion the total energy cost among the entities. Equation 8.1 and 8.2 define $\Pi$ for a common and individual resource system respectively:

$$
\begin{gathered}
\Pi(E(N), N, \ldots)=\left(\pi_{1}, \pi_{2}, \ldots, \pi_{n}\right) \text { in } R^{n} \\
\Pi\left(E_{1}(N), \ldots, E_{n}(N), N, \ldots\right)=\left(\pi_{1}, \pi_{2}, \ldots, \pi_{n}\right) \text { in } R^{n}
\end{gathered}
$$

An apportionment policy should completely apportion the total energy cost: $\sum_{i \in N} \pi_{i}=E(N)$ and $\sum_{i \in N} \pi_{i}=\sum_{i \in N} E_{i}(N)$ for common and individual resource respectively. This is referred to as efficiency in the next section.

\subsubsection{Game theory for energy apportionment}

Cooperative game theory has been extensively employed as a method to model and solve cost division problems. We show that it can be employed to determine the energy contribution of the entities in the system.

A transferable utility game (TU-game) in coalitional form is defined by a set of players $N$ (the entities) and a characteristic function $c$ that models cost. A coalition is a subset $S \subseteq N$, where $N$ is the grand coalition and $\emptyset$ is the empty coalition. The characteristic function assigns a cost to each coalition $S, c: 2^{N} \rightarrow \mathbb{R}$, where $c(\emptyset)=0$ by convention. We employ the system energy function $E$ as the characteristic function $c$ to model the energy consumption of the system. A $n$-player game is defined by the pair $(N, E)$, where the players will divide $E(N)$ given the power structure in the grand coalition expressed by the characteristic function.

Properties of characteristic functions and their structure classify the games providing insights about the usage of apportionment policies. A cooperative game is monotone if $E\left(S_{1}\right) \leq E\left(S_{2}\right)$ when $S_{1} \subseteq S_{2} \subseteq N$. A 
game is subadditive if $E\left(S_{1}\right)+E\left(S_{2}\right) \geq E\left(S_{1} \cup S_{2}\right)$ given $S_{1}, S_{2} \subseteq N$ and $S_{1} \cap S_{2}=\emptyset$, which means that the coalitions have an incentive to cooperate since they lower the costs together. An essential game is characterised by $E(N)<\sum_{i \in N} E(\{i\})$. Finally, a game is concave if $E\left(S_{1} \cup\{i\}\right)-E\left(S_{1}\right) \geq$ $E\left(S_{2} \cup\{i\}\right)-E\left(S_{2}\right)$ for $i \in N, S_{1} \subset S_{2} \subseteq N \backslash\{i\}$. This implies that the incentive for joining a coalition increases when the coalition grows.

Given all possible divisions of the total cost, a single point solution concept for a cooperative game $(N, E)$ provides a unique vector representing the cost division (i.e., apportionment) to each player based on a given criteria. Solution concepts developed for cooperative TU-games can be employed as apportionment policies and its resulting $\pi$ can fulfil certain properties. Efficiency states that the total cost is completely apportioned: $\sum_{i \in N} \pi_{i}=E(N)$. We consider efficiency a minimum requirement for an apportionment policy, and thus, for our analysis all the policies satisfy the efficiency property.

Individual rationality, $\pi_{i} \leq E(\{i\}), \forall i \in N$, implies that a single player has lower or equal cost while cooperating than being alone. An apportionment is an imputation if it satisfies efficiency and individual rationality. We refer to the set of apportionments which satisfy individual rationality and efficiency for a given $E$ as the imputation set $I(E)$.

The game and apportionment properties will be used to compare different energy apportionment policies.

\subsection{Energy apportionment policies}

We first categorise energy apportionment policies based on the type of input information. An energy-based policy (EB) apportions the system energy using different constructs with energy as the only type of input information. A surrogate-based policy (SB) may employ any type of information except energy. A hybrid policy would combine energy and surrogates.

This section presents 10 different policies. We survey 5 of the most used energy-based apportionment policies, propose 2 new policies originated from cooperative game theory and formalise 3 existing surrogate-based policies not described mathematically in earlier work. We briefly explain each policy and provide a brief description of application examples. The application of the policies and their different contexts are explained in section 9.3 (related works). Table 8.1 summarises the 10 energy apportionment policies and describes their intuition.

\subsubsection{Energy-based policies}

This section describes 7 energy-based policies. All policies can be formulated for common and individual resource systems, but in order to simplify the presentation we present them using the common resource notation. 
Table 8.1: Summary of the 10 energy apportionment policies and their intuition.

\begin{tabular}{|c|c|c|c|}
\hline Nr. & Name & Type & Description \\
\hline 1 & Equal division & EB & $\begin{array}{l}\text { Apportion the total energy consumption } \\
\text { equally to all entities. }\end{array}$ \\
\hline 2 & $\begin{array}{l}\text { Proportional } \\
\text { to isolation }\end{array}$ & $\mathrm{EB}$ & $\begin{array}{l}\text { Apportion proportionally to each entity's } \\
\text { isolated energy. }\end{array}$ \\
\hline 3 & $\begin{array}{l}\text { Marginal } \\
\text { contribution }\end{array}$ & EB & $\begin{array}{l}\text { Apportion proportionally to each entity's } \\
\text { marginal contribution. }\end{array}$ \\
\hline 4 & $\begin{array}{l}\text { Isolation energy } \\
\text { and remainder }\end{array}$ & EB & $\begin{array}{l}\text { Isolated consumption and apportion the rest } \\
\text { equally. }\end{array}$ \\
\hline 5 & Shapley value & EB & $\begin{array}{l}\text { Apportion the average marginal contribution } \\
\text { with respect to all permutations on the set of } \\
\text { entities. }\end{array}$ \\
\hline 6 & Nucleolus & EB & $\begin{array}{l}\text { Minimise the unhappiness of the most unhappy } \\
\text { entity. }\end{array}$ \\
\hline 7 & $\begin{array}{l}\text { Least square } \\
\text { nucleolus }\end{array}$ & EB & $\begin{array}{l}\text { Minimise the variance of all entities' } \\
\text { unhappiness. }\end{array}$ \\
\hline 8 & $\begin{array}{l}\text { Proportional } \\
\text { to activity }\end{array}$ & SB & $\begin{array}{l}\text { Apportion proportionally to each entity's } \\
\text { activity, using some notion of activity } \\
\text { (surrogate). }\end{array}$ \\
\hline 9 & Time slicing & SB & $\begin{array}{l}\text { For each time interval apportion equally } \\
\text { among the active entities. If no entity is } \\
\text { active apportion equally among all entities. }\end{array}$ \\
\hline 10 & Last active & SB & $\begin{array}{l}\text { For each time interval apportion equally } \\
\text { among the active entities. If no entity is } \\
\text { active apportion to the last active entity. }\end{array}$ \\
\hline
\end{tabular}

EB: Energy-based apportionment policy. SB: Surrogate-based apportionment policy.

Isolated energy consumption: Energy consumption of an entity if this was alone in the system (see section 8.1.1). Marginal contribution of an entity: Energy when an entity is running in the system minus when it is not running.

Policy 1 - Equal division: The total energy consumption is divided equally by the number of entities:

$$
\pi_{i}=\frac{E(N)}{|N|}
$$

This apportionment policy is regarded as the simplest policy and has been proposed in the context of energy-efficient buildings [97] as well as in energy accounting for wireless sensor networks [83].

Policy 2 - Proportional to isolation: The resulting apportionment is proportional to the isolated energy consumption of each entity. The policy is the same as the pro-rata division of $E(N)$ considering the isolated or stand-alone energy:

$$
\pi_{i}=\frac{E(\{i\})}{\sum_{j \in N} E(\{j\})} E(N)
$$

The energy consumption of a multicore CPU is shared by the different entities in proportion to their estimated energy usage in a single core [217]. 
The same idea has been also proposed to share the cost of heating, ventilation, and air conditioning (HVAC) systems [243].

The most basic version of this policy is proposed for mobile application energy profiling, where the contribution of an application is assumed to be its consumption in isolation $\pi_{i}=E(\{i\})[152,161]$. However, the basic version does not satisfy efficiency since the total energy consumption is not always the sum of the isolated energy consumption of the entities, and thus we only consider the normalised version of the policy.

Policy 3 - Marginal contribution: This policy considers that the contribution of an entity to the total consumption is its marginal contribution to the system $E(N)-E(N \backslash\{i\})$, i.e., the total consumption when the entity is running minus the consumption when the entity is not running. The policy is normalised using all marginal contributions:

$$
\pi_{i}=\frac{E(N)-E(N \backslash\{i\})}{\sum_{j \in N}[E(N)-E(N \backslash\{j\})]} E(N)
$$

This policy was proposed for operating systems to account for runtime energy consumption of individual system hardware and software entities in multicore systems [216,217].

Similarly to Policy 2, a basic version of this policy was proposed in the area of graphical user interfaces [278], where an entity is prescribed its marginal contribution to the system $\pi_{i}=E(N)-E(N \backslash\{i\})$. This basic version does not satisfy efficiency since the sum of marginal contributions is not necessarily equal to the total energy consumption.

Policy 4 - Isolation energy and remainder: The policy prescribes the allocation of costs as a function of the energy consumption in isolation plus proportionally dividing the additional cost/benefit of non-isolated use.

$$
\pi_{i}=E(\{i\})+\frac{E(N)-\sum_{j \in N} E(\{j\})}{|N|}
$$

The usage of this policy is originated in the energy-efficient building community [97], where the personal consumption is estimated first and the rest is divided evenly (e.g., base energy load of building elements such as HVAC). In the context of wireless sensor networks, the energy cost of messages received by a certain task is prescribed to that task and the rest (e.g., synchronisation or message loss) is shared evenly [116].

Policy 5 - Shapley value: Given the marginal contribution of $i$ to a coalition $S \subseteq N \backslash\{i\}$ defined by $E(S \cup\{i\})-E(S)$, the Shapley value computes the average marginal contribution of $i$ averaging over all the possible sequences through which the grand coalition can be built from the empty coalition [226]:

$$
\pi_{i}=\sum_{S \subseteq N \backslash\{i\}} \frac{|S| !(|N|-|S|-1) !}{|N| !}[E(S \cup\{i\})-E(S)]
$$


The Shapley value is a well known single value solution from game theory applied to many cost sharing problems in computer science $[151,224]$ as well as in other fields [78]. Recently it has been argued to be the ground truth for energy accounting in mobile devices [63].

Policy 6 - Nucleolus: Given an imputation $\pi=\left(\pi_{1}, \pi_{2}, \ldots, \pi_{n}\right)$, i.e., an efficient apportionment satisfying individual rationality, the satisfaction of a coalition from apportionment $\pi$ is measured by the excess:

$$
e(S, \pi)=E(S)-\sum_{i \in S} \pi_{i}
$$

Let $\theta(\pi)$ denote a vector that contains the excess for each coalition $S \subseteq$ $N$ in non-decreasing order given an imputation $\pi$. The nucleolus is the apportionment $\pi$ which maximises $\theta(\pi)$ over all the other imputations $\pi^{*}$ in the imputation set $I(E)$ using the lexicographic ordering $\left(\geq_{L}\right)[220]$ :

$$
\pi \mid \theta(\pi) \geq_{L} \theta\left(\pi^{*}\right) \text { for all } \pi, \pi^{*} \in I(E)
$$

The nucleolus is commonly computed by solving a sequence of linear programming (LP) problems [144]. The prenucleolus computes an apportionment following the same method for the set of all efficient apportionments, providing an apportionment when the imputation set is empty.

Policy 6 is proposed in this paper for energy apportionment due to its interesting properties (see section 8.4).

Policy 7 - Least square nucleolus: For a given imputation $\pi$ the average excess $\bar{e}$ is:

$$
\bar{e}(\pi)=\frac{1}{2^{|N|}-1} \sum_{S \subset N} e(S, \pi)
$$

The least square nucleolus (LSN) is the imputation that minimises the following: $\sum_{S \subset N}(e(S, \pi)-\bar{e}(\pi))^{2}$, i.e., an imputation whose associated excesses are closest to the average excess under the least square criterion [214]. The least square prenucleolus (LSP) is the efficient apportionment that follows the same method.

The computation of the LSN is performed in two steps: (1) LSP $\pi^{P}$ is calculated using Equation 8.9,

$$
\pi_{i}^{P}=\frac{E(N)}{|N|}+\frac{1}{|N| 2^{|N|-2}}\left[|N| \sum_{S: i \in S} E(S)-\sum_{S \subset N}|S| E(S)\right]
$$

followed by (2) a recursive algorithm that computes the LSN $(\pi)$ using the LSP apportionment $\pi^{P}$ as input. For each step in the algorithm a 0 is given to the entities with a negative excess in some of the earlier steps, dividing the aggregated negative excess from the previous step evenly among rest of the entities. The algorithm stops when no entity gets a negative excess, which results in an apportionment $\pi$ which is an imputation.

To the best of our knowledge LSN has not been used for energy apportionment policies. 


\subsubsection{Surrogate-based policies}

This section employes the time model described in section 8.1.1 to formalise 3 previously proposed surrogate-based policies. These policies are selected because we believe that they are good representatives of surrogate-based policies.

Policy 8 - Proportional to activity: Given two events that signify that an entity becomes active and inactive respectively $(V=\{0,1\}$, where $v=1$ for active and $v=0$ for inactive), the policy apportions energy proportionally to the activity time. Let $s_{i}$ be the signal of events of entity $i$, the apportionment policy is defined as:

$$
\pi_{i}=\frac{a\left(s_{i}\right)}{\sum_{j \in N} a\left(s_{j}\right)} E(N)
$$

where $a(s): S \rightarrow \mathbb{R}$ is a function that provides the total active time of entity $i$ by performing a sum of the periods between events with values 1 and 0 . This policy has been proposed to model the occupancy of a building to divide its energy cost among the occupants [97].

Policy 9 - Time slicing: This policy considers that multiple entities may be active simultaneously and there exist idle time. Given the sequence of events $\left(e_{1}, e_{2}, \ldots, e_{m}\right)$ temporally sorted in $\mathbf{s}$, a sequence of intervals $J$ is computed. An interval $j \in J$ is given by the delimiting events' time points $\left[t_{j}, t_{j+1}\right)$, where $e_{j}=\left(t_{j}, v\right), e_{j+1}=\left(t_{j+1}, v^{\prime}\right)$ and $t_{j}<t_{j+1} . T$ is the period between the first and last event.

The Boolean function $a(i, j): N \times J \rightarrow\{0,1\}$ denotes if an entity $i$ is active during interval $j$ or not. Function $f(i, j): N \times J \rightarrow[0,1]$ divides an interval among the active entities proportionally, or evenly if the entities are inactive.

$$
f(i, j)= \begin{cases}\frac{1}{|N|} & \text { if } \sum_{i \in N} a(i, j)=0 \\ \frac{a(i, j)}{\sum_{i \in N} a(i, j)} & \text { otherwise }\end{cases}
$$

This function is used to define a policy as described in Equation 8.11:

$$
\pi_{i}=\frac{1}{T} \sum_{j \in J}\left[f(i, j)\left(t_{j+1}-t_{j}\right)\right] E(N)
$$

The policy is used to apportion a micro controller's consumption in a scenario of a distributed database over a network of sensors [116] and a building's energy to tenants [236]. Also, this policy is used for apportionment of a single core processor [79].

Policy 10 - Last active: Given the intervals $J$, the energy consumption for each interval is apportioned among the active entities. During any interval that all entities are inactive, the energy consumed during the interval is assigned to the last active entity. 
For each interval $j$ the energy consumption is denoted by $E(N, j)$, where $\sum_{j \in J} E(N, j)=E(N)$. The Boolean function $l(i, j): N \times J \rightarrow\{0,1\}$ denotes if entity $i$ is the last active entity of interval $j$ by simply keeping track of the latest "become active" event $(v=0)$.

$$
g(i, j)= \begin{cases}l(i, j) & \text { if } \sum_{i \in N} a(i, j)=0 \\ \frac{a(i, j)}{\sum_{i \in N} a(i, j)} & \text { otherwise }\end{cases}
$$

Policy 10 is defined using function $g(i, j)$ :

$$
\pi_{i}=\sum_{j \in J} g(i, j) E(N, j)
$$

The policy provides incentives to aggregate the active events of the different entities. This policy is used to apportion the energy tails created by inactivity timeouts for different smartphone components [173].

The next sections compare the presented 10 energy apportionment policies based on 3 criteria: input information, computational complexity and fairness. Section 8.3 analyses the input information and computational complexity, whereas in section 8.4 we analyse the fairness of the different policies employing five formal criteria.

\subsection{Input and computational complexity}

The presented policies require different amounts of input information to perform the apportionment and obtaining this information might be harder depending on the domain of the input and the system. In addition, the amount of input information has an impact on the computational complexity of the policies. We analyse and compare the computational complexity of the policies and provide pointers to efficient solutions in the literature for the most complex policies.

Table 8.2 summarises the comparison of the policies using the different criteria and we describe the details of the comparisons next.

\subsubsection{Required input information}

We compare the domain of the input information for the different policies, i.e., surrogate or energy information, and then analyse the amount of information needed for each policy.

\section{Information type}

We categorised the policies based on input type: energy consumption or surrogates. Dividing energy consumption based on energy information appears as a natural choice: if the cost function is known, it is natural to use 


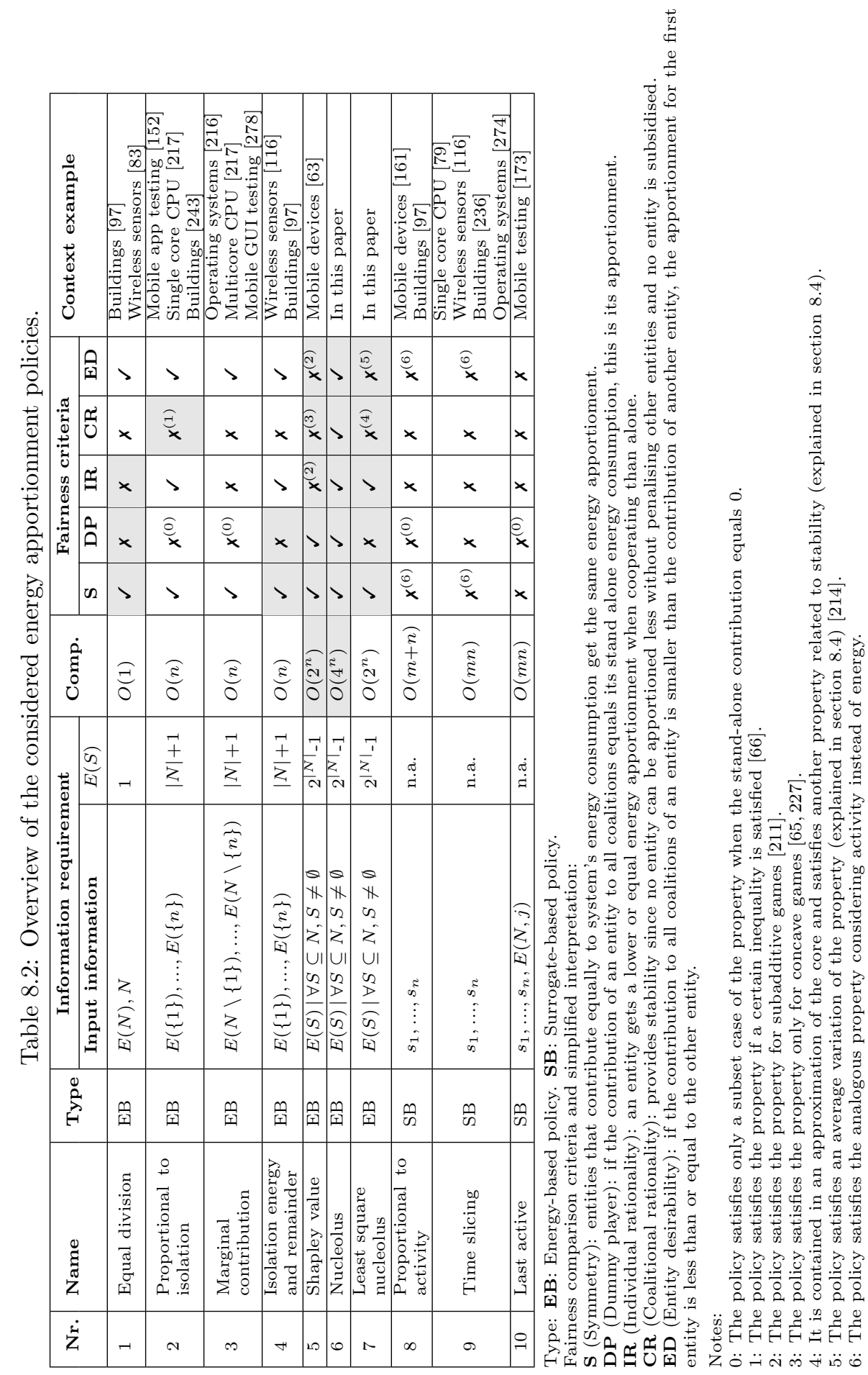


it to apportion the cost. However, while obtaining $E(N)$ is straightforward, measuring or estimating $E(S) \mid S \subset N$ is complex (if not impossible) given the shared nature and lack of visibility of energy consumption in current systems. We observe that obtaining $E(N \backslash\{i\})$ seems qualitatively harder than $E(\{i\})$ since the first already considers the energy resulting from the use of a shared resource, whereas the second one considers the energy in isolation without any interaction with the other entities. Approximations of $E(S)$ range from accurate energy models [152,173] (e.g., EnergyBox) to measuring some of the observable $S \subset N$ and approximating the unobserved values using regression techniques [63].

Surrogate-based policies perform energy apportionment by translating the problem to another domain outside energy consumption. This can simplify the apportionment problem and the data acquisition (e.g., surrogate information is easily accessible using performance counters in computers [31] or external activity sensors in buildings [46, 218]). However, the surrogate input does not necessarily reflect how energy is consumed in the system. For example, the amount of data sent does not reflect the energy consumed in the wireless interface but it is easily accessible.

\section{Information quantity}

Columns 4 and 5 in Table 8.2 show the amount of needed information for each policy. Policy 1 (Equal division) employes the minimum input quantity: $E(N)$ and $N$. Policies 2, 3 and 4 (Proportional to isolation, Marginal contribution and Isolation energy and remainder respectively) require the energy consumption of $|N|$ subsets. Policies 2 and 4 consider the energy in isolation $E(\{i\})$ and 3 considers the system energy excluding a single entity $E(N \backslash\{i\})$. Policies rooted in cooperative game theory (policies 5, 6 and 7 ) present the highest information requirement, requiring the energy consumption of all the subsets of $N\left(2^{|N|}\right)$ to build the game.

Apart from $E(N)$, the input the surrogate-based policies is from another domain than energy consumption. Surrogate-based policies (policies 8-10) require at least a signal $s_{i}$ per entity ( $n$ signals) with the events collected from the system. Additionally, Policy 10 (Last active) requires $E(N)$ per interval.

Thus, depending on the available information for a system some policies seem more appealing than others. It is worth mentioning that there exist ways to approximate games with incomplete information (e.g., missing information about the consumption of some subsets) $[146,186]$.

\subsubsection{Computational complexity}

This section presents the computational complexity of the policies. We analyse the general complexity and provide pointers for problem specific complexity reduction for the most complex policies. Column 6 in Table 8.2 shows the complexity of each policy. The size of the input (information 
quantity) is a highly influential aspect in the computational complexity of the policies.

\section{General complexity}

Policies 1 to 4 show low complexity. Policy 1 (Equal division) performs a single operation and its complexity is $O(1)$. Policies 2, 3 and 4 (Proportional to isolation, Marginal contribution and Isolation energy and remainder) are computed in $O(n)$ given the sum of $|N|$ elements. The computational complexity of Policy 5 (Shapley value) increases exponentially with the number of entities $O\left(2^{n}\right)$. Policy 6 (Nucleolus) is the most complex policy, computed by solving a sequence of at most $|N|$ number of linear programming (LP) problems of decreasing dimension [144] or a single large-scale LP problem with $O\left(4^{n}\right)$ constraints [190]. The complexity of Policy 7 (Least square nucleolus) is $O\left(2^{n}\right)$ due to two steps: (1) the calculation of LSP using Equation 8.9 involves iterating over the $2^{n}$ coalitions, and (2) a maximum of $|N|$ operations are performed by the algorithm to compute LSN.

The complexity of Policies 8-10 depends on the number of events $m$ of all the entities and the functions employed. The complexity of Policy 8 (Proportional to activity) is $O(m+n)$ due to analysing $m$ events when using function $a(s)$ and the sum of $|N|$ entities. Policy 9 (Time slicing) and 10 (Last active) employ $m-1$ intervals and for each interval and entity, and the policies compute the apportionment using functions $f(i, j)$ and $g(i, j)$ respectively, leading to a $O(m n)$ complexity.

\section{Optimisations}

There exist approximation techniques for Policy 5 (Shapley value) where the computational complexity increases linearly with the number of players $[42,76]$. There are also exact solutions that take advantage of some particular game representation to reduce the complexity (e.g., $O(n)$ and $O\left(n^{2}\right)$ for a tree [148] and weighted graph representation respectively [60]).

Efficient solutions have been found for the Policy 6 (Nucleolus) for specific game structures, such as a tree structure where it is computed in $O\left(n^{3}\right)$ [148] or a weighted graph in $O\left(n^{2}\right)$ [60]. However, NP-hardness has been shown for other specific game representations (e.g., minimum spanning tree game [74]). Reducing the computation requirement for the nucleolus is an ongoing work.

To sum up, the computational complexity is important to consider when performing energy apportionment of systems, especially with a large number of entities. Policies 5-7 exhibit higher complexity than the rest of policies. 


\subsection{Fairness in energy apportionment}

Except Policy 5, the application of all the presented policies to apportion energy consumption has been promoted as fair by the authors using them (see Table 8.2) with no formal justification. We analyse fairness by first introducing a formal notion of fairness that leverages game theory in section 8.4.1. Section 8.4.2 describes in detail the fairness properties satisfied by each policy, which is summarised in Table 8.2.

\subsubsection{Fairness criteria}

In this section we propose that fairness can be characterised by selecting five different criteria. These criteria capture properties that are important when apportioning energy consumption in an intuitive and rational manner. We introduce and motivate each criteria for energy apportionment:

- Symmetry: if $E(S \cup\{i\})=E(S \cup\{j\})$ for all $S \subseteq N \backslash\{i, j\}$, then $\pi_{i}=\pi_{j}$. If the marginal contribution of $i$ and $j$ to all coalitions is the same, their apportionment is the same. Symmetry is a desirable property for an apportionment policy since two entities that contribute equally to the energy consumption of the system should intuitively receive the same apportionment.

- Dummy player: if $E(S \cup\{i\})-E(S)=E(\{i\})$ for all $S \subseteq N \backslash\{i\}$, then $\pi_{i}=E(\{i\})$. If the marginal contribution of entity $i$ to all the coalitions is the same as its stand-alone value, then the latter is her value.

The property reflects the fact that the consumption of an entity in the system can be independent of the other entities, and thus it is intuitive to assume that if the entity does not impact the energy consumption of the system or the rest of the entities when sharing the resource, this entity should be prescribed its stand-alone cost. The property also includes the case of when $E(\{i\})=0$, which should intuitively lead to a prescription of no cost. This basic case of dummy player is also known as null player.

- Individual rationality: This property was briefly introduced in section 8.1.3. We recall that an apportionment is individually rational if $\pi_{i} \leq E(\{i\}), \forall i \in N$. An apportionment policy satisfies individual rationality if it results in such an apportionment when it exists. Recall that when such an apportionment exists it is called an imputation, and the set of such imputations is denoted $I(E)$. It is well known that $\sum_{i \in N} E(\{i\}) \geq E(N)$ iff $I(E) \neq \emptyset$. Hence, the property is formally defined as: if $I(E) \neq \emptyset$ then $\pi_{i} \leq E(\{i\}), \forall i \in N$.

We argue that individual rationality is an appropriate fairness criteria since: (1) When all the entities contribute to lower the total energy 
consumption compared to the sum of entities' stand-alone consumption, it is unintuitive to prescribe an entity more that it would consume alone, and (2) individual rationality provides incentives to cooperate since every entity's apportionment in the system is lower than what its energy consumption would have been in isolation.

- Coalitional rationality: An apportionment is coalitionally rational if $\sum_{i \in S} \pi_{i} \leq E(S) \forall S \subseteq N$. An apportionment policy satisfies coalitional rationality if it results in such an apportionment when it exists. In game theory, the set of apportionments that satisfy coalitional rationality is referred to as the core ${ }^{1}$ of a game denoted by $C(E)$. Then, coalition rationality is formally defined as:

if $C(E) \neq \emptyset$ then $\sum_{i \in S} \pi_{i} \leq E(S) \forall S \subseteq N$.

This property is relevant since it implies that no entity or coalition is subsidised: if one entity would be prescribed less cost in a coalitionally rational apportionment, another entity would be penalised, i.e., the apportionment is Pareto optimal [65]. Additionally, this property is attractive for energy apportionment in cooperative systems, since selecting a coalitionally rational apportionment leads to the case where no coalition could reach a better result than in the grand coalition. This introduces a stability notion by avoiding any incentive to divide the grand coalition.

- Entity desirability: For the final criterion we adapt the player desirability criterion introduced by Rosenbusch [211]. Entity $i$ is said to be more desirable than entity $j(i \succeq j)$ if the energy consumption contribution of $i$ to all coalitions that exclude both entities is less than or equal to the one that $j$ would contribute: $E(S \cup\{i\}) \leq$ $E(S \cup\{j\}) \forall S \subseteq N \backslash\{i, j\}$. The criterion states that a more desirable entity gets an apportionment less than or equal to the apportionment of the less desirable entity: $i \succeq j \Longrightarrow \pi_{i} \leq \pi_{j}$.

We refer to $i$ and $j$ as comparable if $i \succeq j$ or $j \succeq i$. We believe that the criterion is intuitive, since a lower contribution of an entity than another should be reflected in the apportionment.

\subsubsection{Fairness comparison}

The fairness criteria column in Table 8.2 shows whether a policy always satisfies the symmetry, dummy player, individual rationality, coalitional rationality and entity desirability properties. The 21 shaded cells in the table out of the 50 relate to known results based on previous extensive analysis from game theory literature. Table D.1 in the appendix provides the references to the existing results tracing each property to each policy.

\footnotetext{
${ }^{1}$ Even though the core presents desired properties, we do not consider the core as an apportionment policy and use it as a comparison criterion since the core is rarely unique and may be empty.
} 
The other 29 outcomes are our contributions. These results strictly follow from the respective fairness property and policy definitions. The detailed analysis of the policies in this section is structured per property, where we provide the intuition of why a certain policy satisfies or does not satisfy each property. We refer the interested reader to the electronic appendix (attached at the end of this manuscript) where all the proofs can be found. We also refer to each of our proofs in the text.

\section{Symmetry}

All policies satisfy symmetry except for the surrogate-based policies (Policies 8,9 and 10). The proofs for the symmetry property can be found in section D.1 of the appendix.

Since the Equations 8.3-8.9 of policies 1 to 7 do not consider any information other than the value of the system energy function $E$, two entities contributing with the same energy to all coalitions always get the same apportionment. For example, Policy 1 (Equal division) trivially satisfies symmetry since it always apportions the same value to all entities, or Policy 2 (Proportional to isolation) divides the stand-alone energy by the same value for all entities (Theorem 1). In a similar way, Policy 3 (Marginal contribution) satisfies symmetry since the denominator is equal for all entities (Theorem 2).

Policies 8, 9 and 10 (surrogate-based policies) do not satisfy symmetry since activity and energy are not necessarily correlated. Thus, even if two entities are symmetric in terms of energy, this is not reflected in the apportionment policies 8, 9 and 10 which consider activity as input (Theorems 3, 4 and 5 respectively).

\section{Dummy player}

Policies 5 and 6 (Shapley value and Nucleolus), are the only policies satisfying the dummy player criterion. The general reason for not satisfying the property is that the policies perform a certain operation that prevents an entity from getting its isolated energy consumption. The proofs for the dummy player property can be found on section D.2 of the appendix.

The normalisation factor of Equations 8.4 and 8.5 is the main reason for not satisfying dummy player for Policy 2 (Proportional to isolation) and Policy 3 (Marginal contribution) (Theorems 6 and 7 respectively). Even if an entity contributes with its isolated energy consumption $E(\{i\})$ to all coalitions, Policy 2 and 3 normalise the result and thus the apportionment does not always result in that isolated energy consumption.

Surrogate-based policies (8, 9 and 10) do not always satisfy the criterion even in the case where the activity function is equal to the system energy function. Policy 8 (Proportional to activity) employes a normalisation factor (similarly to Policy 2) (Theorem 8). Policy 9 (Time slicing) divides the idle energy equally among all entities (Theorem 9). Policy 10 (Last active) 
prescribes a greater energy consumption to the last active entity in the system (Theorem 10).

Finally, policies 2, 3, 8 and 10 satisfy the special case when the contribution of an entity is 0 to all coalitions. For surrogate-based policies, it is intuitive that if an entity does not have a surrogate attribute in a scenario (e.g., it does not have any activity), it does not consume energy in isolation and vice versa. Hence, $\mathrm{a}(\mathrm{i})=0$ iff $\mathrm{E}(\{\mathrm{i}\})=0$.

It is easy to verify this so called null player property for Policy 2 (Proportional to isolation), Policy 3 (Marginal contribution) and Policy 8 (Proportional to activity) by looking at Equations 8.4, 8.5 and 8.10: if the contribution of an entity is 0 , the entity is prescribed no energy since $E(\{i\}), a(i)$ and $E(N)-E(N \backslash\{i\})$ become 0 respectively, and thus $\pi_{i}=0$ (Theorems 11,12 and 13 respectively). For Policy 10 (Last active) if an entity is never active it cannot be the last active entity and thus it is prescribed no energy (Theorem 15).

\section{Individual rationality}

Policy 2 (Proportional to isolation), Policy 4 (Isolation energy and remainder), Policy 6 (Nucleolus) and Policy 7 (Least square nucleolus) always result in an individually rational apportionment when it exists. The proofs for the individual rationality property can be found on section D.3 of the appendix.

Policies 2 and 4 (Proportional to isolation and Isolation energy and remainer), always satisfy individual rationality since the apportionment is performed by considering the isolated energy consumption $E(\{i\})$ (Theorems 16 and 18).

However, Policy 3 (Marginal contribution) does not always satisfy individual rationality mainly due to Equation 8.5 does not consider the energy consumption of $E(\{i\})$ (Theorem 17). Policy 5 (Shapley value) does not always satisfy this property, but if the resulting game based on the energy consumption values $E(S)$ is subadditive Policy 5 will satisfy individual rationality.

Similarly, the surrogate-based policies (policies 8, 9 and 10) do not satisfy the property since the activity of an entity is not necessarily correlated with energy consumption (Theorems 19, 20 and 21).

\section{Coalitional rationality}

Policy 6 (Nucleolus) is the only apportionment policy that satisfies coalitional rationality when such a solution exists. Coalitional rationality implies individual rationality when $|S|=1$, and thus Policies $1,3,8,9$ and 10 that do not always satisfy individual rationality do not satisfy coalitional rationality either.

Policy 2 (Proportional to isolation) does not always satisfy coalitional rationality, but it will satisfy the criterion if the following restrictive inequality 
holds [66]:

$$
\frac{\sum_{i \in S} E(\{i\})}{\sum_{i \in N} E(\{i\})} \leq \frac{E(S)}{E(N)} \text { for all } S \subseteq N
$$

Policy 4 (Isolation energy and remainder) does not always satisfy coalitional rationality since cost of the coalitions is not considered in Equation 8.6 (Theorem 22). Policy 5 (Shapley value) does not always satisfy coalitional rationality. However, if the resulting game based on the energy consumption values $E(S)$ is concave, Policy 5 satisfies the property [211].

Finally, Policy 7 (Least square nucleolus) is not always coalitionally rational, but other works have shown that it is always contained in an approximation of the core (least-core), which describes approximately stable outcomes $[145,153]$. Additionally, Policy 7 is the only solution ensuring that on average all entities are equally treated if each entity evaluates the excess of the coalitions that the entity belongs to: $\sum_{S \mid i \in S} e(S, \pi)=\sum_{S \mid j \in S} e(S, \pi), \forall i, j \in N$. The egalitarian principle towards coalitions becomes egalitarian towards the entities too, and thus this solution contributes to stability.

\section{Entity desirability}

Policies 1, 2, 3, 4 and 6 always satisfy entity desirability. Our proofs for entity desirability can be found in section D.5 of the appendix.

Policy 1 (Equal division) trivially satisfies it since the policy always prescribes $\pi_{i}=\pi_{j}$ (Theorem 23). Policies 2 and 4 (Proportional to isolation and Isolation energy and remainder) always satisfy entity desirability. A more desirable entity has a lower (or at least equal) isolated consumption $E(\{i\})$ than a less desirable entity, and thus Equations 8.4 and 8.6 prescribe always a lower (or at least equal) apportionment (Theorems 24 and 26 respectively). Policy 3 (Marginal contribution) always satisfy entity desirability since a more desirable entity has a lower marginal contribution (Theorem 25).

Policy 5 (Shapley value) does not always satisfy the property for the general case, but the policy will always satisfy entity desirability when the game is subadditive [211].

Policy 7 (Least square nucleolus) does not always satisfy entity desirability, but instead it satisfies an attractive property: aggregated entity desirability $^{1}$. Instead of comparing the marginal contribution of two entities to each coalition, this property compares the total sum of the marginal contributions to all coalitions and prescribes a smaller cost if the sum of an entity's contribution is smaller than or equal to another entity's contribution [214]:

$$
\begin{gathered}
\text { if } \sum_{S \mid i, j \notin S} E(S \cup\{i\})-E(S) \leq \sum_{S \mid i, j \notin S} E(S \cup\{j\})-E(S) \\
\text { then } \pi_{i} \leq \pi_{j} \text { for all } i, j \in N
\end{gathered}
$$

\footnotetext{
${ }^{1}$ This property is called Average Marginal Contribution Monotonicity, but we rename it to keep term consistency in the evaluation.
} 
The approach of Policy 7 considers all the coalitions equally important.

Finally, the surrogate-based policies (8,9 and 10) do not always satisfy entity desirability since less activity does not necessarily mean lower energy consumption (Theorems 27, 28 and 29).

\subsubsection{Summary of the fairness analysis}

Table 8.2 shows that Policy 6 (Nucleolus) provides the strongest fairness since it satisfies all properties. The intuition is that Policy 6 is defined to minimise the unhappiness of the most unhappy entity in the system.

Policy 5 (Shapley value) is an excellent and fair alternative provided that the system energy function $E$ is always subadditive for the system. If subadditivity does not hold, the only advantage of this policy compared to the other ones is that it satisfies the dummy player property. Thus, subadditivity is a precondition for a system to employ the full potential of the Shapley value policy.

Policy 1 (Equal division) and Policy 3 (Marginal contribution) satisfy only symmetry and entity desirability, which makes other policies such as Policy 2 (Proportional to isolation) more attractive. Policy 2, is preferred to Policy 4 (Isolation energy and remainder) since Policy 2 additionally satisfies the subset case of dummy player when the contribution of an entity is 0 . Policy 2 is an attractive choice given the symmetry, individual rationality and entity desirability criteria, which provide incentives and a notion of fairness based on comparing two entities in the system.

Policy 7 (Least Square Nucleolus) is very interesting alternative given its more egalitarian approach to fairness and the additional fairness properties that it satisfies compared to Policy 5 or 6 . In addition, all coalitions are equally treated by the policy (i.e., all the complaints have the same weight) and it contributes to stability.

The detailed analysis of the above 10 policies pinpoints that surrogate policies do not provide guarantees in terms of fairness from the energy perspective defined as the 5 criteria. If the antecedents of the fairness properties are defined in terms of the activity function $a$ instead of $E$, e.g., for symmetry $a(S \cup\{i\})=a(S \cup\{j\})$ for all $S \subseteq N \backslash\{i, j\}$, Policies 8 (Proportional to activity) and 9 (Time slicing) satisfy activity-based symmetry and entity desirability. Nevertheless, the two criteria providing incentives (individual rationality and coalitional rationality) inherently require energy related concepts, and thus activity needs to be correlated to energy, otherwise surrogate-based policies can provide misleading incentives.

\subsection{Summary of the trade-offs and guidelines}

In this section we provide an overview of the presented analysis considering the different evaluation criteria and the identified trade-offs. Section 8.5.1 summarises the major trade-offs between fairness and the other evaluation 
criteria. Section 8.5.2 discusses the importance of analysing the system energy function $E$, and finally section 8.5.3 provides some high-level guidelines to select an energy apportionment policy given the purpose of the apportionment.

\subsubsection{General trade-offs}

The major trade-offs identified in the analysis are the following:

- Fairness vs. information type: We have distinguished between energy-based and surrogate-based energy apportionment policies based on the type of input information. Surrogate information (e.g., resource access time or data sent) is in general easily accessible compared to energy-based information which usually needs to be estimated.

However, we have shown that some fairness properties (dummy player, individual rationality and coalitional rationality) cannot be satisfied by a surrogate-based policy. In addition, if the surrogate information is not highly correlated with the energy information, the surrogate policies will not always satisfy the other fairness properties (symmetry and entity desirability). Table 8.2 reflects this issue showing that none of the surrogate-based policies satisfies any of the 5 fairness properties in the general case.

- Fairness vs. computational complexity: The amount of input information is tightly related to the computational complexity of a policy, which leads to a trade-off between fairness and computational complexity.

Policy 1 (Equal division) provides the lowest complexity but also weak fairness. Policies 2, 3 and 4 present low information requirement (a single value per entity) leading to a low computational complexity, but none of these policies satisfies the 5 fairness properties. We identified Policy 2 (Proportional to isolation) as an interesting policy in the trade-off between fairness and computational complexity: it provides reasonable fairness (providing symmetry and entity desirability) as well as incentives (individual rationality) in linear time.

Surrogate-based policies (Policies 8, 9 and 10) are not very attractive since they do not satisfy any fairness property and the computation requirement is still similar to Policies 2,3 and 4 .

Satisfying the additional fairness properties requires more input information (e.g., to compare coalitions) leading to higher computational complexity. Policy 6 (Nucleolus) satisfies all fairness properties, and Policies 5 and 7 (Shapley value and Least square nucleolus) have a well defined notion of fairness. However, the size of the input grows exponentially with the number of entities for the game-theoretic policies. Moreover, coalitional rationality (satisfied by Policy 6) relates to 
an inherently hard problem. Determining whether there exists a set of coalitionally rational apportionments is NP-complete $[52,53]$.

Thus, the computational cost of satisfying all fairness properties is high, and lowering the computational cost requires selecting a smaller set of fairness properties. Alternatively, one could apply a high complexity approach to a problem with a small set of inputs. Moreover, as pointed out in section 8.3.2, there are efficient algorithms to compute the game-theoretic policies for restricted classes of games.

To sum up, fairness comes at at cost of having to measure or estimate energy consumption as well as computational complexity.

\subsubsection{Properties of the system energy function}

The system energy function $E$ models the energy consumption of the system and its values are either measured or estimated. In section 8.1.3 we introduced some common properties used to classify $E$ (e.g., subadditive or concave). Knowing the class of $E$ is highly valuable for Policy 5 (Shapley value) since it satisfies relevant fairness properties only for some of the $E$ categories.

While subadditivity is often an assumption for some problems in the game theory literature $[70,226]$, it is difficult to say how likely it is that the system function $E$ is subadditive for energy systems. If $E$ is subadditive, the energy consumption of two coalitions (resp. entities) together is not higher than the sum of their isolated consumption. This implies that being together in the system is always advantageous, which implies that the interaction of the entities in the system is efficient. While analysing whether an instance of $E$ is subadditive is intuitively system dependent, we provide some illustrative examples that result in a subadditive $E$ and a non-subadditive $E$ respectively:

- Subadditive: Consider a system similar to the one presented in the introduction section, where major contribution to the energy consumption is due to an inactivity timer triggered after performing some activity. For example, a Secure Digital card (SD card) consumes energy for some seconds after each write/read operation [173]. If two applications use the SD card concurrently, the total energy is smaller than sum of the stand-alone energy of the applications (overlapping energy tails). If the applications use it separately, the consumption is simply the sum of the stand-alone energy. Thus, the system is subadditive. Other examples following a similar intuition are a water kettle or a TV [207], where the usage overlap by several users intuitively results in a subadditive $E$.

- Non-subadditive: Consider the $3 \mathrm{G}$ wireless interface studied in this thesis which is often characterised by two main consuming states depending on the utilisation. The drivers of some WiFi interfaces use a 
similar mechanism [192]. Small amounts of data are sent in a low consuming state, but when the data sent for a period of time is higher than a given threshold, the interface is moved to a high energy state. The consumption of two processes sending small amounts of data together can lead to the high energy state, whereas sending data separately would result in a lower consumption due to using the low energy state separately. Thus, this system is not always subadditive.

Consider another simple example from the energy-efficient building area, a refrigerator, whose compressor is activated periodically in order to maintain the desired temperature in the hysteresis region [46]. Opening the refrigerator increases the need of starting the compressor earlier. For this system, the result of two people opening the refrigerator separately can easily result in a non-subadditive $E$ since the entities alone would consume less than together.

Concavity implies subadditivity, and hence, from the discussion above, it follows that some real-world examples of $E$ may not satisfy concavity.

To sum up, we believe that further work is needed to analyse the properties of $E$ for different types of systems. It is also interesting to analyse the properties of $E$ for complex systems composed of different subsystems (e.g., a mobile device has a SD card as well as some wireless interfaces).

\subsubsection{Selection guidelines}

In this section we outline the most significant criteria that impact the selection of a policy for energy apportionment. Based on our analysis and the previous discussion, the following criteria serve as guidelines in order to select a policy:

- Surrogate vs. energy-based policy: Where applicable, we believe that energy-based policies are more appropriate than surrogate-based ones. Highly accurate energy models and measurement techniques are already available for many systems and obtaining better estimates of the $E$ values is an ongoing work. This will facilitate the information acquisition for the apportionment.

- Number of entities and real-time requirements: The number of entities in the system plays an important role on the computational complexity, and this can drastically impact the timing requirements for energy apportionment. In general, complex algorithms are not fit for large number of entities, especially in real-time applications of apportionment. However, they may make sense in offline analysis settings. Analysing the running time of the apportionment policy implementations in different systems as well as their energy cost is an interesting future direction. 
- The purpose of apportionment: We classify the purpose of apportionment into three main categories: informing, profiling and incentivising.

- Informing: When the purpose of the energy apportionment is simply to inform about the overall energy consumption of the entities (e.g., rank the most consuming entities), there is no strict need for a complex policy. We believe that Policy 2 (Proportional to isolation) is a good fit for this purpose since it includes a fairness notion where each entity is compared in a one-to-one fashion (symmetry, null player and entity desirability).

- Profiling: This applies when the purpose of the apportionment is to accurately profile the energy usage (via testing) by entities in a system, e.g., for improving the efficiency of applications or software optimisation. Symmetry and entity desirability are necessary properties for this purpose and the dummy player property is desirable. Table 8.2 shows that Policy 5 (Shapley value) and Policy 6 (Nucleolus) are good choices for this purpose.

- Incentivising: Energy apportionment can provide incentives to reduce the energy consumption, especially when the entities of the system can influence their energy consumption. Basic incentives can be realised by selecting a policy that satisfies individual rationality, such as the simple Policy 2 (Proportional to isolation). It provides incentives to reduce the stand-alone consumption of an entity, since this will be apportioned less than its stand-alone consumption when the system is efficient. For example, it can incentivise application developers to reduce the stand-alone energy of their applications.

Satisfying coalitional rationality is recommended when the purpose is to incentivise entities to efficiently cooperate in the system in addition to reduce their stand-alone consumption. For example, two applications can be incentivised to reduce their consumption together (e.g., by synchronising transmission patterns) because they are apportioned no more than their stand-alone consumption, but also no more than their consumption together. Coalitional rationality is desirable in systems where multiple dynamic entities can join or leave the system (e.g., device-to-device clusters in $5 \mathrm{G}[33,35])$. In such cases incentives are the key to cooperation.

Thus, a system can benefit from a strong incentive structure of Policy 6 (Nucleolus) which satisfies individual and coalitional rationality, at the cost of computational complexity. A more egalitarian fairness notion towards all coalitions of Policy 7 (Least square nucleolus) is also an interesting alternative when users are involved (e.g., energy-efficient buildings). 
Finally, if the subadditivity and concavity of $E$ can be shown, Policy 5 (Shapley value) can also provide incentives.

Thus, depending on the purpose of energy apportionment and the system specifics some policies can be a better fit than others. We have identified that Policy 2 (Proportional to isolation) provides an interesting tradeoff between computational complexity and fairness while still providing basic incentives. Policy 6 (Nucleolus) and Policy 7 (Least square nucleolus) are the most interesting properties in order to provide stronger incentives, and Policy 5 (Shapley value) and Policy 6 (Nucleolus) are a good fit to profile systems. Finally, it is clear that analysing $E$ is crucial for employing Policy 5 (Shapley value).

\subsection{Summary and discussion}

In this chapter we categorised, compared and analysed a total of 10 different energy apportionment policies in terms of required information, computation complexity and fairness. Among the 10 policies, we formalised 3 policies not described mathematically earlier, and proposed two novel policies based on cooperative game theory.

Our work shows that cooperative game theory provides an excellent framework and solutions for addressing the energy apportionment problem. Selecting an appropriate policy plays a role in providing incentives for energy efficiency to the entities in the system.

Unfortunately, there is no known ground truth for the general apportionment problem and therefore energy-based policies are no better than surrogate-based ones. However, we have pinpointed that providing incentives and stability requires energy related concepts and that an energy apportionment policy needs to reflect the inner workings of the system leading to energy consumption. Thus, employing the system energy function $E$ in the apportionment seems a more reasonable alternative.

Our results demonstrate that there is a trade-off between fairness and the other evaluation criteria. The strongly fair policies based on game theory have the highest information requirement and computational complexity, which can be less practical in some contexts (e.g., high number of entities in the system). Depending on the context, a simpler policy like Policy 2 (Proportional to isolation) can achieve a satisfactory compromise by sacrificing some fairness properties but drastically reducing information requirement as well as complexity and still providing basic incentives.

The characteristics and properties of the system energy function $E$ should be analysed since some policies assume a certain properties (e.g., Shapley value) and sometimes different policies can lead to identical results. In the latter case selecting the simplest policy among the equivalent ones is recommended. 
In our context, the selection of the best apportionment policy depends on the purpose of the energy apportionment, e.g., profile their application or provide incentives to applications from the operating system. We have preliminary pinpointed that $E$ is not always subadditive for a wireless interface (e.g., 3G), and thus studying the system energy function for the different wireless interfaces is suggested as part of future works. Finally, it is interesting to note that analysing the game formed by the $E(S)$ values helps to determine the efficiency of an application in isolation (by looking at $E(\{i\})$ ) and the efficiency of the interactions with the other applications (by looking at $E(S) \mid i \in S)$. While the developers can maximally influence the energy consumption in isolation, their influence regarding the interaction between the applications is limited. The operating system or lower level mechanisms can analyse the inefficient interactions and provide means to improve the coordination as well as potentially provide incentives. 
"They say a little knowledge is a dangerous thing, but it's not one half so bad as a lot of ignorance."

Terry Pratchett

\section{9 \\ Related Works}

Recent years have seen a growing interest in measuring and reducing the energy consumption in mobile devices towards green networking. Mobile network operators have become interested in the energy saving approaches as well, mostly due to increasing cost of their networks [73]. However, new proposals often adopt an infrastructure-centric approach $[108,142,143,165$, 279] rather than considering a holistic approach including user terminals. A comprehensive description of the challenges of green cellular networks is provided on a survey by Hasan et al. [96]. This chapter provides a survey of the related work focused on energy consumption of data communication at the user end, and thus infrastructure-centric approaches are not considered. The same reasoning is applied also for other technologies such as $\mathrm{WiFi}$, where the infrastructure-centric approaches are not considered [85,112,140,213].

The general categories of the taxonomy were briefly described in section 2.3. The extended taxonomy is shown in Fig. 9.1. Our contributions are within the shaded categories, and thus we compare our work in the corresponding sections. The other categories are not required for understanding the contributions of our work. They provide pointers to different approaches to reduce the consumption of mobile devices and are included here as complementary material. Some related works appear in more than one section due to their contributions to different areas (e.g., a measurement study and solution proposal).

It is worth mentioning that some researchers have collected large datasets to analyse general mobile device and application usage, such as type of applications, user interactions or battery charging patterns [36, 75, 170, 249, 254]. While these are very useful to study the interactions with applications, 


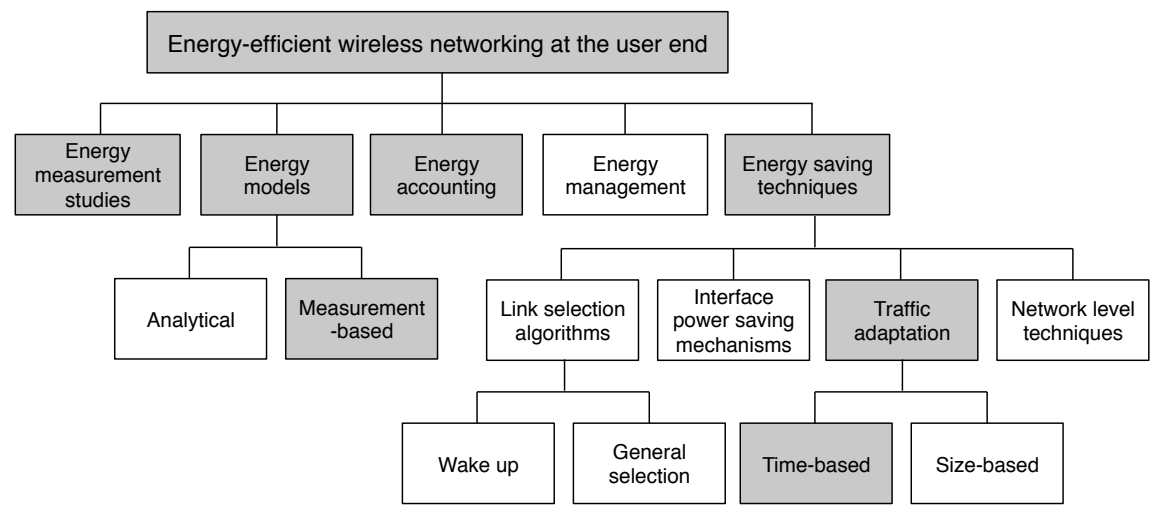

Figure 9.1: Taxonomy of wireless communication energy at the user end.

the transferred data information is often limited by a low sampling rate and they do not study communication energy in detail.

\subsection{Energy measurement studies}

Measurement studies typically aim at revealing inefficiencies, discovering the footprint characteristics of wireless interfaces and providing guidelines for energy-efficient operation and design. Experimental setups for energy consumption studies on mobile devices either use built-in software in mobile devices like Nokia Energy Profiler (NEP) [24,90,176,179,191,252] or external power meters [77, 198, 208, 223, 255].

We categorise the studies based on the radio technology used in their work. Some works focus on various technologies in order to compare them.

\subsubsection{Cellular communication}

An influential measurement study by Balasubramanian et al. [24] reports the categorisation of three different energy components in cellular networks: ramp, transfer and tail. The tail component is the most significant and is caused by the inactivity timer statically set by the network operators. Our previous work [17] refines this study by performing physical measurements using a cellular modem that isolates the energy for data transfers. Their work uses bulk data transfers, which do not characterise the impact of the data pattern on the energy consumption.

A detailed study of the tail energy overhead in $3 \mathrm{G}$ cellular networks is performed by Qian et al. [198] on user data traces retrieved from a network operator in 2009. The work is extended [197] pointing out how different applications inefficiently utilise the radio resources due to their data pattern. Both works provide an excellent ground for understanding the impact 
of the operator-configured timers and RLC data buffer thresholds on the theoretical user battery discharge.

Schulman et al. [223] and more recently Ding et al. [61] explore the impact of the network coverage on the $3 \mathrm{G}$ energy consumption. Their measurements show that mobile devices consume more energy when sending data under poor link conditions.

Puustinen et al. [191] measure the impact of unwanted Internet traffic on the energy consumption for $3 \mathrm{G}$ and propose blocking traffic and fast dormancy as potential solution. Ukhanova et al. [245] study the energy consumption of constant bit rate video communication over $3 \mathrm{G}$ and propose a RRC state machine parameter selection method to decrease the uplink energy consumption. Their work shows that by adapting the video quality and transmission power one can achieve energy savings. Cuervo et al. [56] state that $3 \mathrm{G}$ has a high cost to perform energy-aware code offloading.

Perrucci et al. [181] focus their study on the energy consumption difference of $2 \mathrm{G}$ and $3 \mathrm{G}$ according to common usage of the networks, such as SMS, voice calls and data traffic. They suggest to use SMS compression for long messages, which could be also applicable to other type of data. They propose to perform vertical handover between $2 \mathrm{G}$ and $3 \mathrm{G}$ depending on the usage pattern. Our previous work [17] confirms the benefit of using $2 \mathrm{G}$ instead of $3 \mathrm{G}$ for small amounts of data.

The main advantage of our measurement setup compared to the above works is that we are able to isolate the energy consumption of data transfers by performing measurements in a $2 \mathrm{G} / 3 \mathrm{G} / \mathrm{GPS}$ module.

The energy consumption characteristics of LTE have been also studied. Huang et al. [106] study the energy consumption of LTE using physical measurements in a smartphone connected to a commercial network. Using a dataset of 5 month traces of 20 users they show that LTE is less efficient than $3 \mathrm{G}$ given the contribution of the long energy tails. Siekkinen et al. [231] provide an interesting background in terms of LTE energy consumption. They question whether the LTE measurements by Huang et al. [106] were performed with DRX enabled since they show significant differences in terms of energy savings. A comprehensive explanation of the $4 \mathrm{G}$ (WiMAX and LTE) power saving techniques is provided by Kim et al. [119].

The benefit of using EnergyBox to efficiently complement physical measurements is shown in earlier chapters. Our work has contributed by performing energy consumption studies in the context of mobile games, location sharing applications and instant messaging applications described in Chapters 4,6 and 7 respectively. Thus, we devote the last part of this section to mention and compare some related work in the three contexts.

- Mobile gaming: Mobile games have not been subject to extensive attention in terms of energy studies and most of them consider WiFi instead of $3 \mathrm{G}$. A previous study has shown that the $3 \mathrm{G}$ communication contributes to more than $50 \%$ of the total energy consumed for a mobile game (e.g., Angry Birds) over 3G communication [173]. 
ARIVU [32] is an energy-aware middleware designed to reduce the energy consumption of the $\mathrm{WiFi}$ interface when playing online multiplayer games. Prokova et al. [189] compare the average power usage of Android games with and without advertisements connected via WiFi. Our work focuses on the 3G energy consumption and shows that game-specific background data is a major contributor in addition to ad-related traffic.

The following two works study the gaming usage patterns. Patro et al. [174] study data collected from a massively multiplayer game over a three year period and show, among other results, that around $70 \%$ of the sessions are 20 minutes or shorter. Another study of a single player game by Obermiller and Bayless [167] points out that half of the players played the game only a few times and for 15 minutes. We use this earlier data to justify that our selection of the duration of each play is reasonable given that we had to perform each session manually.

Recent works focus on improving the performance aspects, such as improving mobile graphics by performing GPU offloading to the cloud [57] or reducing the impact of latency on the game [128]. Other studies focus on matchmaking systems for online multiplayer games $[139,276]$.

- Location sharing services: The literature regarding energy consumption of location-based services is mostly focused on reducing the cost of the actual location retrieval rather than in the location sharing: Kjærgaard [120] describes the trade-off between energy consumption and location accuracy by measuring the energy consumption of different location-based services. EnTracked [122] is a configurable system that schedules position updates when tracking GPS-enabled devices using motion sensor. The system can be configured to minimise energy consumption by duty-cycling system components (e.g., GPS) while maintaining the real error below a given application limit. EnLoc [54] selects the localisation source (GPS, cellular or Wi-Fi) to use given the optimal localisation accuracy for a given energy budget. ALoc [136] trades off energy consumption and accuracy, replacing GPS by WiFi and cell-tower triangulation. Foll et al. [82] study contextual state (e.g., sitting in the office) update mechanisms, trade-off between number of messages and quality of the context. Zhuang et al. [281] propose an adaptive location-sensing framework involving alternative location-sensing mechanisms to GPS.

Whereas the above works mostly focus on reducing the energy consumption of retrieving the location or context, our work covers the location consumption gap studying the actual cost of sharing the location using $3 \mathrm{G}$. Sharing the location was previously identified as a high energy cost by Kjærgaard [120]. We experimentally compare two protocols and data formats used for location sharing purposes showing a significant difference in energy consumption. 
- Instant Messaging: Even though IM has a great popularity and a large number of users, little work has been done to understand its energy consumption at the user end. Most IM related works focus either on users' social interactions [88], security [222] or analysing the IM traffic generated in desktop-oriented machines [111,130,265].

While presence updates have been already studied in desktop-oriented machines $[256,265]$ and in the mobile context $[50,51,149]$, the cost of the typing notification feature or the impact of consecutive messages has not attracted attention so far. Thus, our work pinpoints the high cost of using the notification mechanism and a great energy consumption difference between IM applications as well as energy-inefficient user usage patterns identified using our dataset.

\subsubsection{WiFi communication}

Rice and Hay [208] measure the WiFi energy consumption in a variety of smartphones. Their experimental methodology for measuring mobile devices is an inspiration for our work. Using their measurement framework they study the energy consumption of the Dynamic Host Configuration Protocol process and data transmission in 802.11 networks in different devices, reaching the conclusion that mobile devices would make the most out of a transmission with big message sizes. Their work reports the power consumption differences due to hardware and software generations.

Several works focus on studying the energy consumption of WiFi based on bulk data transmissions $[24,84,256]$. Studies using bulk data transmissions do not capture the impact of adaptive PSM on energy consumption. Xiao et al. [263] show that the download of videos using WiFi is more energyefficient than in $3 \mathrm{G}$, suggesting a tailor-made solution for streaming using WiFi. Various works $[140,193,213]$ study the different adaptive PSM mechanisms in previous generation devices. A recent study by Pyles et al. [192] shows the threshold to switch between PSM and CAM for different devices.

Atheros [18] reports that the energy consumption decreases when increasing the data rate. The features included in the $802.11 \mathrm{n}$ standard leading to higher data rates (e.g., modulation and coding schemes, channel bonding, frame aggregation or multiple-input and multiple-output) are studied in terms of energy $[92,132,257]$. The impact of RSSI in the data rate and energy consumption is studied showing a significant energy increase under poor signal strength [61]. Finally, several works measure the high cost of the WiFi ad hoc mode dominated by the idle consumption [77,176,240,252]. The above works are a great input to further improve the WiFi model included in EnergyBox. 


\subsubsection{Bluetooth}

While Bluetooth is not the main technology used in the thesis, we provide few references to interesting studies $[67,84,155,183,242]$. These can be used to add Bluetooth models to EnergyBox.

Finally, several other measurement-based energy consumption studies focused on mobile devices are not considered here due to their non-networking related nature. The readers interested in other energy aspects than communication for single mobile devices are referred to the survey by VallinaRodriguez and Crowcroft [248] or to the recent survey by Hoque et al. [104].

\subsection{Energy models}

Energy models abstract the behaviour of the devices characterising the mechanisms that consume energy. Some works focus on specific mechanisms whereas others attempt to model the total energy consumption. Energy models are typically either measurement-based or analytical. The focus of this section is on the measurement-based models since EnergyBox our proposal in this regard (presented in Chapter 3).

\subsubsection{Measurement-based models}

We start by describing works that focus on characterising communication energy. We also include works that characterise the energy consumption of mobile devices since they consider communication energy in the models.

Communication energy: Balasubramanian et al. [24] model the energy consumption of $3 \mathrm{G}$ and $\mathrm{WiFi}$ using linear regression based on their bulk data download measurements. Given the amount of data to be sent in burst, their lineal model provides the energy consumed. This simple model does not capture the impact of the data pattern on the energy consumption and it is dependent on their measurements (e.g., device and network settings). Oliveira et al. [169] use a state-based model for 3G modelling the RLC buffer occupancy as fixed data rate (kbps) without performing any validation of their model.

Qian et al. [198] propose a finite state machine 3G model to study the impact of the inactivity timers on the energy consumption using a large traffic dataset. The radio resource usage application profiler for $3 \mathrm{G}$ presented by Qian et al. [197] is the work closest to our EnergyBox for 3G. Their profiler is a similar tool to EnergyBox that at the time of its publication was not available to other researchers. Our $3 \mathrm{G}$ state-based model includes a low activity mechanism implemented by some operators, thereby improving the accuracy of the model. Moreover, our EnergyBox captures different RRC state machines within a single parametrised one. 
The work by Harjula et al. [95] creates a device-specific power profile for different messaging intervals based on device measurements on a Nokia 95 for a single operator. Their model uses indicators such as average packet size and signalling frequency that need to be extracted from the data transmissions. Instead, EnergyBox directly works on data traces and derives the RRC states of different networks simulating the RLC buffer thresholds and state transitions. Our approach is more general and simplifies energy consumption studies.

The power consumption of the DCH state is modelled as a linear equation using measurements on a Nokia N900 given the uplink and downlink power consumption for different of packet sizes [245]. Their device-specific model can be easily applied to model the different data rates in the DCH state for EnergyBox.

RILAnalyzer [246] focuses on recording the low-level radio information (e.g., RRC states) directly from the radio chipset firmware without analysing energy. Their tool is available for a single chipset and requires root privileges in a similar way to the Voodoo RRC Tool [253], which also provides the RRC states for the same chipset. EnergyBox accurately emulates the RRC states to obtain the energy consumption using input traffic traces and provides repeatability for experiments. However, RILAnalyzer and Voodoo RRC Tool could be used to obtain the network parameters from different networks as an input to EnergyBox.

Huang et al. [106] compare the power and throughput characteristics for WiFi, 3G and LTE using measurement-based models for a single phone. The models include a linear throughput-power curve measured in the phone since the LTE power substantially increases with the data rate.

Xiao et al. [264] present a detailed power level model for the $802.11 \mathrm{~g}$ interface. The modelled data pattern based on traffic burstiness was only validated by simulating TCP download and upload traffic based on the amount of data transferred. In comparison, our adaptive PSM model is validated against a set of representative application traces.

Mobile device models including communication: Several works aim at quantifying the total energy expenditure of a device considering the wireless interfaces as well as other components such as CPU, screen or audio interfaces. A recent study by Pathak et al. [173] presents a system-callbased power modelling for smartphones, including device dependent $3 \mathrm{G}$ and WiFi models. Tracing system calls typically requires modifications to the operating system (e.g., root access), whereas we adopt a generic approach based on packet captures, which are generally available ${ }^{1}$. Ding et al. [61] extend the system-call-based model to include the RSS impact on the power consumption of $3 \mathrm{G}$ and WiFi by performing a detailed measurement study. Their measurement results are useful means to include the RSS impact on power consumption for EnergyBox.

PowerProf [121] is an automatic method to create energy models using a set of training measurements that exercise the different components of a 
mobile device. The resulting models are dependent on the training data, which are likely to miss the underlying mechanisms of cellular and WiFi interfaces. Their method requires the definition of a wide set of relevant tests to exercise the different components. Instead, EnergyBox directly models the lower layer operation of $3 \mathrm{G}$ and WiFi. A combination of both methods would provide versatility and accuracy by merging automatic model generation and expert knowledge.

Wilke et al. [258] propose a generic unit testing framework for profiling the energy consumption of applications. Their follow up work [260] proposes to compare the energy consumption of similar Android applications to derive energy labels using physical measurements. Their testing framework would benefit from using EnergyBox as generic trace-based communication energy measurement tool for multiple operators and devices.

The work by Mittal et al. [152] proposes an energy simulation tool for Windows phones leveraging component models taken from the literature. For $3 \mathrm{G}$, they measure different inactivity timers for different operators and a perform basic signal strength modelling by changing the fixed power values in each state.

Zhang et al. [275] propose an online energy estimation and model generator implemented on Android. Hardware components are trained to generate device specific models using system variables (e.g., LCD brightness level). For $3 \mathrm{G}$, a single operator is modelled by sending packets based on a previous methodology [198]. The WiFi model is based on a finite state machine using the number of packets sent/received per second, uplink channel and data rate. Their work proposes to substitute the external power measurements by the battery discharge curve and the current battery voltage. Since the battery discharge curve also varies from battery to battery, their approach requires to characterise the battery discharge for each battery separately.

Dong et al. [64] propose to automatically self-generate a system energy model within a device using the smart battery interface. Similarly to energy modelling in desktop computers, their work employs system statistics and memory usage and complements them with the battery interface readings. However, smart battery interfaces that provide current measurements are often not available in many devices.

\subsubsection{Analytical models}

These approaches often model the behaviour of a specific mechanisms of a wireless interface and suggest guidelines to select parameters for potential optimisations. Different studies focus on studying the energy consumption due to the inactivity timers and state transitions of UMTS [126, 268], the discontinuous reception mechanism (DRX) for UMTS [267] and LTE $[22,37,150,280]$ and specific mechanisms for other interfaces such as WiFi

\footnotetext{
${ }^{1}$ Android phones provide traffic statistics that can be captured every millisecond and packet level capture is available without root permission from Android 4.0 on.
} 
[271] and Bluetooth [270]. The named works are limited to modelling application traffic (e.g., web and streaming), whereas EnergyBox is applicable to arbitrary data traces captured from any real application.

\subsection{Energy accounting}

Energy accounting aims at quantifying, analysing and reporting the energy consumption of different entities or activities of interest in the system. Our work in Chapter 8 studied the energy apportionment problem and provided a theoretical foundation for fairness leveraging cooperative game theory. We showed that fairness is a relevant criterion and pinpoint that, as in cooperative game theory, there is no best solution in general. We surveyed the different policies and compared them. Additionally, we propose the usage of two apportionment policies with strong fairness notions which have not previously been used for energy apportionment. Finally, we provide guidelines to select a policy based on the information requirement, computational complexity and fairness properties and suggest that the analysing structure of the $E$ function for a system is decisive.

This section reviews the application and implementation of the surveyed energy apportionment policies. For each application area we briefly explain each energy apportionment implementation and report which policies they employ. Since very little work is available in the literature regarding energy accounting in the context of wireless communication, we broaden the scope to include the most relevant works. The works are categorised in four different contexts: processing and operating systems, wireless sensor networks, energy-efficient buildings and mobile devices. These works provide evidence that the energy apportionment problem is present in different contexts where the same policies have been used by different researchers.

\subsubsection{Processing and operating systems}

PowerScope [79] maps energy consumption to program structure by employing physical power measurements combined with kernel level system activity information. The work studies a single-core CPU, and thus attributes a process the energy consumed when the process is being executed. Since a single process can be executed simultaneously, the process is allocated its consumption based on the time slice when it was scheduled (similar to Policy 9 - Time slicing). This work does not consider cases where the activity of entities in the system is not instantaneously reflected in the power trace (e.g., energy tails).

A follow up work by Bellosa [28] proposes JouleWatcher, a similar eventdriven resource profiler to attribute energy consumption to individual threads. Even though the work does not clearly state the problem of multi-entity apportionment and no apportionment policy is proposed, it refers to surrogate information such as performance-monitoring information. 
Neugebauer and McAuley [161] argue that energy accounting of shared resources is a requirement for energy management. They discuss the difficulties in estimating each entity's consumption due to asynchronous energy overheads (e.g., energy tails). They suggest that the apportionment should be done in proportion to utilisation-based surrogates, i.e., Policy 8 (Proportional to activity). Example of surrogates are the access time to a resource, number of pixels used in a display or amount of data exchanged with the network.

ECOSystem [274] is an energy-centric operating system which employs energy accounting for the CPU, disk and network card. The running time of the process is used to apportion the CPU consumption assuming that the CPU consumes a fixed amount of power (Policy 9 - Time slicing). The active access of the disk is computed using surrogates when write or read operations occur. The consumption due to asynchronous operation (e.g., spin-up, spinning and spin-down) is proportionally divided by the tasks that employed it between the spin-up and spin-down events. This is similar to Policy 9 (Time slicing) considering the spin-up and spin-down as events. Finally, the energy cost of the network interface is attributed based the amount of data transmitted and received.

Ryffel et al. [217] and $\mathrm{LEA}^{2} \mathrm{P}$ [216] extend the idea of PowerScope to multi-core systems. Policy 3 (Marginal contribution) is proposed as fair without any formal justification to attribute the total energy consumption of the system to processes. The system employs power measurements of different hardware elements (e.g., CPU, GPU or hard drive) combined with indirect information based on performance counters. However, they mention that obtaining the energy consumption values for Policy 3 (Marginal contribution) is not feasible for some system components such as CPU since processes run simultaneously. Estimating the energy consumption of a process in isolation (in a single CPU) is proposed to overcome the limitation, and thus Policy 2 (Proportional to isolation) is employed to estimate the apportionment cost of each process for the multi-core processor.

Joulemeter [114] is a system to attribute the energy consumption of a cloud server to the running virtual machines (VMs). The energy consumption of each VM is estimated based on measurement-based energy models. It is argued that the apportionment is correct if the sum of the estimated energy is equal to the total measured energy (i.e., efficiency), which is not always the case. They consider the energy in isolation as the apportionment for each VM (similar to Policy 2 - Proportional to isolation).

\subsubsection{Wireless sensor networks}

Quanto [83] is an energy profiler for network nodes which combines energy metering hardware and program activity information to quantify the energy consumption for user defined activities. They use the notion of activity as a logical set of operations whose resource usage is grouped together. An 
entity can adopt the same notion in our work.

Quanto estimates the energy breakdown of the different components and then system quantifies the resource consumption of the activities. A singleactivity device is considered leading to a straightforward apportionment, but the authors mention that the apportionment for a multiple-activity device is a policy decision. They preliminary select to divide the energy consumption equally (similar to Policy 1 - Equal division).

Kellner [116] considers the context of dynamic sensor networks where various applications can query a sensor network at any point in time. The work proposes an online energy accounting system employing measurementbased finite state machine (FSM) models to attribute energy consumption to tasks. Since energy consumption does not only depend on the active usage (e.g., energy tails or between uses), the author identifies several ways to divide the remainder energy. Policy 4 (Isolation energy and remainder) is applied to message reception where the energy consumption of the messages that cannot be attributed directly to any task is equally shared. Policy 9 (Time slicing) is used to divide the energy consumption of the micro controller considering active or inactive tasks.

\subsubsection{Energy-efficient buildings}

A first and influential work by Hay and Rice [97] formulates the problem of attributing the energy consumption of a building or organisation to individual users. They argue that it can provide incentives to reduce the total consumption and identify two requirements that every policy should satisfy: completeness and accountability. The first one refers to efficiency, whereas the second states that actions of a user should have a maximal effect on their own share and a minimal on the rest of users. The entity desirability property is related to accountability, where a more consuming entity will get prescribed a higher energy consumption.

Their work proposes Policy 1 (Equal division) as the most basic policy. This is further extended using occupancy time as a surrogate of energy resulting in a version of Policy 8 (Proportional to activity). Policy 4 (Isolation energy and remainder) is then proposed by first calculating the personal load of a user and dividing the rest evenly. Their efforts focus on accurately estimating the personal load employing sensors.

WattShare [236] seeks to attribute the energy consumption of a shared building to individual occupants (personal apportionment) using Policy 9 (Time slicing). Considering only the availability of the total energy consumption measured at the smart meter, their work proposes to employ smartphones carried by the occupants to perform the apportionment. Their approach uses the following information: indoor localisation based on the received signal strength indicator of $\mathrm{WiFi}$, microphone data from the smartphones, appliance-to-room mapping and appliances' power profiles. Using the collected information the system associates the users to the detected 
electrical events using time slices and divides the total energy cost.

Tsao et al. [243] investigate how to attribute the energy consumption of centralised heating, ventilation and air conditioning system to different rooms in a building. They propose to employ Policy 2 (Proportional to isolation) and argue that it satisfies efficiency and accountability. There is no evidence showing that their apportionment approach satisfies the two properties.

\subsubsection{Mobile devices}

Pushed by short battery lifetimes energy profiling has attracted a great attention in mobile devices to improve software energy efficiency. Section 9.2.1 already presented some of the works present in this area from a different perspective. We now focus describing the energy accouting approach and the used energy apportionment policy.

When analysing the energy consumption of applications in Chapters 4, 6 and 7 we do not seek to perform apportionment, we have assumed the energy contribution of an application to be its consumption in isolation. We isolated the traffic of the application under test to make sure that only the traffic from the application is contributing to the total energy consumption of the wireless interface. Other studies assume the same in order to profile the energy consumption due to communication $[197,208]$ which is similar to the basic version of Policy 2 (Proportional to isolation).

Similarly, WattsOn [152] is a system to estimate the energy consumption by an application at the development environment which reuses and extends previously developed FSM-based energy models. This work considers that the energy consumption of the application is the total energy consumption of the system when only the application is running (i.e., similar to Policy 2 - Proportional to isolation). Zhang et al. [275] develop activity-based power models (FSM and linear functions) using measurements in order to attribute the energy consumption of each component to the different processes in the system. Their work does not consider the asynchronous energy consumption such as energy tails.

Bugu [133] is an application level service which aims to profile the applications running in client devices by sending consumption statistics to a server. The considered apportionment policy is that an application's consumption is the energy consumption in isolation $(E(\{i\}))$ based on their power model (similar to Policy 2 - Proportional to isolation), which does not always satisfy efficiency. The energy profiling is performed with simple models based on active time and inactive time, which limits the accuracy of their approach.

The characterisation of graphical user interfaces and display energy consumption from hardware, software and application perspective is performed with a focus on optimising the energy consumption of GUI applications and GUI platforms [278]. The work uses Policy 3 (Marginal contribution) to 
obtain the energy contribution of GUIs.

Eprof [173] is an energy profiler for smartphones to support efficient application development. It employs system call-based resource usage information and FSM-based models to attribute the energy consumption to applications (and activities within the application). They discuss different alternatives to attribute the energy consumption of energy tails such as equally (Policy 1 - Equal division) or proportionally (Policy 2 - Proportional to isolation). Finally, they propose the last-trigger policy (Policy 10 - Last active in our work) in order to incentivise the developer to batch energy events. In our work we formalised their proposed policy to analyse its properties.

Zhang et al. [275] develop activity-based power models (FSM and linear functions) using measurements in order to attribute the energy consumption of each component to the different processes in the system. Their work does not consider the asynchronous energy consumption such as energy tails.

The recent work by Dong et al. [63] is the first work considering the application of cooperative game theory in the context of mobile energy accounting. They argue that Policy 5 (Shapley value) is the ground truth for the apportionment problem in mobile systems and provide an approximation of it by approximating the game. Using physical power measurements they define every $10 \mathrm{~ms}$ interval as a game where the energy consumption needs to be attributed. By considering a short interval they attempt to observe the consumption of different coalitions (i.e., observing intervals where only some entities are active) and employ a combination of the observed coalitions with hardware states (for $\mathrm{WiFi}$ and $\mathrm{CPU}$ ) to reduce the variance of the observations. The unobserved coalitions are statistically estimated from historical data. Their results show that other accounting methods differ significantly from their approach.

Recent work has also focused on identifying energy hungry applications by collaboratively collecting usage information from many devices and employing statistical techniques. Carat [168] is a collaborative method which employes coarse-grained client-side information such as battery level and local data (e.g., running processes or device model) sent to a server to detect energy anomalies across many different devices. Following a similar idea E-Loupe [44] aims to collaboratively detect the cause of energy spikes by collecting resource usage and battery state information. These works provide a limited level of energy accounting due to the coarse granularity of the approach which reduces the possibilities of using them in energy management.

Compared to the previous works we focus on analysing the fundamental aspects of fairness in energy apportionment policies rather than application or implementation aspects of the policies. Our work provides an overview of the energy apportionment problem in different research areas in order to unify efforts towards energy efficiency. 


\subsection{Energy management}

Efficiently managing system resources in terms of energy requires to control and determine the energy expenditure of the system and the entities. Energy accounting, and thus energy apportionment too, are key elements to determine the energy consumption of the entities. This section describes approaches considering energy allocation to competing entities in the system, mostly from the operating system and mobile device literature. These rely on energy accounting and apportionment policies. This section is complementary material that illustrates the application of energy apportionment into energy management.

\subsubsection{First energy-aware approaches}

The first energy-aware operating system concepts and attempts for mobile devices (laptops in this case) date from late 90s [69,161]. Ellis [69] advocates that energy consumption should be consider as a first class resource while still meeting performance requirements. Since then, the main approach in energy management is to devise different strategies to control the energy consumption of the system by limiting the energy usage of entities in the system (e.g., applications).

Budget-based energy management (BBEM) allocates an energy budget to entities in the system (e.g., applications) and these will not run if they run out of budget. Setting a target lifetime is also a recurring trend. Nemesis [161], ECOSystem $[69,273,274]$ and Odyssey [80,81] are examples of early operating systems that propose adaptive applications which can tradeoff QoS for lower consumption. These works also pinpointed the need for energy accounting. For example, Odyssey uses PowerScope [79] to build a system's power model which and employs Policy 8 as apportionment policy.

ECOSystem [69, 273, 274] employs BBEM by allocating energy to competing tasks based on a performance and energy consumption tradeoff. Currentcy, the energy currency used by ECOSystem, represents a certain amount of consumed energy. A user-specified battery lifetime target is set and ECOSystem periodically allocates currentcy to tasks represented as resource containers. Resource containers [25] are an operating system resource abstraction used to control the resource consumption. In periods of high demand the currentcy is allocated to competing tasks according to user-specified proportions, and if an application has no currentcy it will not be scheduled.

The Odyssey operating system $[80,81]$ proposes a collaborative relationship between operating system and applications to adapt QoS requirements to meet energy demands using application level adaptation. Odyssey lets the user set a battery lifetime goal and when the energy of the system is not sufficient to reach the goal the operating system solicits the applications to reduce their consumption. The system requires application developers 
to develop adaptation and low-power modes for the applications. PowerScope [79] is used to build a power model of the system.

\subsubsection{Current approaches in mobile devices}

More recently BBEM has been adopted and extended by several works to extend the battery lifetime of mobile devices. The Cinder operating system $[212,215]$ refines the ideas presented in ECOSystem and presents two abstractions called reserves and taps: virtual energy storages based on resource containers and the connection between these storages respectively. Each entity in the system (e.g., application or process) has a reserve of energy which can use to consume system resources and the taps create a resource tree in which each edge limits the rate at which energy is accumulated in the reserves, where the root reserve is the battery. Similarly to ECOSystem, when the energy in the reserve is not enough to perform any activity the process or application will not be run (even if the application is running in the foreground). An additional issue is the complexity on setting the tap values since consumption peaks are common in mobile devices and thus it can decrease QoS.

The complexity of BBEM lies on devising a strategy to define the budgets and the replenishment rate to overcome the potential QoS and QoE burden on the user when applications run out of budget. Prioritising some applications [48], limiting background applications [44] or exploiting context information (e.g., user location) $[49,247]$ are some examples of recent proposals for energy management.

Finally, the theoretical work by Dong [62] formulates energy management as a utility optimisation problem: schedule the different processes to maximise the aggregate utility of all system processes under the energy capacity constraint. BBEM is compared to a novel energy management strategy which dominates by optimal utility value.

\subsection{Energy saving techniques}

Energy saving techniques are categorised in different groups based on their aim and means. The solutions presented in Chapters 5, 6 and 7 are categorised under traffic adaptation techniques and are compared against the other works.

\subsubsection{Traffic adaptation techniques}

Given the footprint characteristics of a wireless interfaces (e.g., data rate or power saving mechanism), energy-aware traffic adaptation techniques aim at adapting regular traffic to create energy-efficient traffic, i.e., adapting the system load. The traffic adaptation is usually performed by shaping the 
traffic over time, reducing the amount of data sent or a mix of both by altering the data pattern using different techniques.

Cellular periodic transfers and background traffic of mobile applications are known to incur a great energy consumption. There exists a vast literature aiming to reduce its energy consumption. Many techniques accommodate different flavours of fast dormancy within their proposed technique due to it is an improvement over the standard, and orthogonal to traffic adaptation techniques.

Additionally, multimedia streaming for mobile devices has also attracted great attention. Hoque et al. [103] provide a survey of energy-efficient solutions which are categorised based on the IP stack layers and divided into traffic scheduling and multimedia content adaptation techniques.

Size-based: Some techniques consider the benefit of sending less data or stripping the redundant data (e.g., compression). The viability of data compression for wireless communication in mobile devices is studied by Wang et al. [255] concluding that it can provide up to $50 \%$ of energy savings by reducing the amount of data transmitted. Stratus [4] combines compression with bursty communication during good signal strength periods. Armstrong et al. [14] propose an infrastructure to support dynamic content updates by only updating the interesting part within Web pages selected based on the client interest reducing the transmission data.

While compression works for big amounts of data, the work presented in Chapter 7 in the area of IM applications has shown that small messages do not benefit from compression. These would require a combination of size and time techniques to compress several messages at the same time.

Time-based: Given that data transmissions can be shifted over time, different techniques enable to create energy-efficient traffic by aggregating transmissions. Traffic aggregation defines the set of techniques that defer the data transmissions within a maximum period until one of them needs to be sent (e.g., user-specified deadline). All the transfers that were postponed until the last moment are sent together reducing the number of state transitions and energy tails at the cost of the delay. Batching refers to grouping several periodic transfers into a single one, i.e., increasing the period of the transmissions. The batching point is usually defined in advance and the applications send their traffic in those time points. Batching and traffic aggregation can lead to different results. The batching points are periodic and non-traffic dependent. However, traffic aggregation techniques usually consider the traffic. Finally, piggybacking is a method where background data transfers can be deferred or moved earlier in time to match the transfers with users' foreground traffic.

Fig. 9.2 shows the conceptual difference between traffic aggregation and batching. For traffic aggregation, each packet is delayed up to a maximum delay and buffered. The packets are sent in burst before the maximum delay. The batching points are defined in advance given a batching interval. 
Then, the packets are shifted in time and sent in those batching points. The example shows how the same time for batching interval and maximum delay can lead to different bursts due to traffic aggregation depends on the packet arrival time.
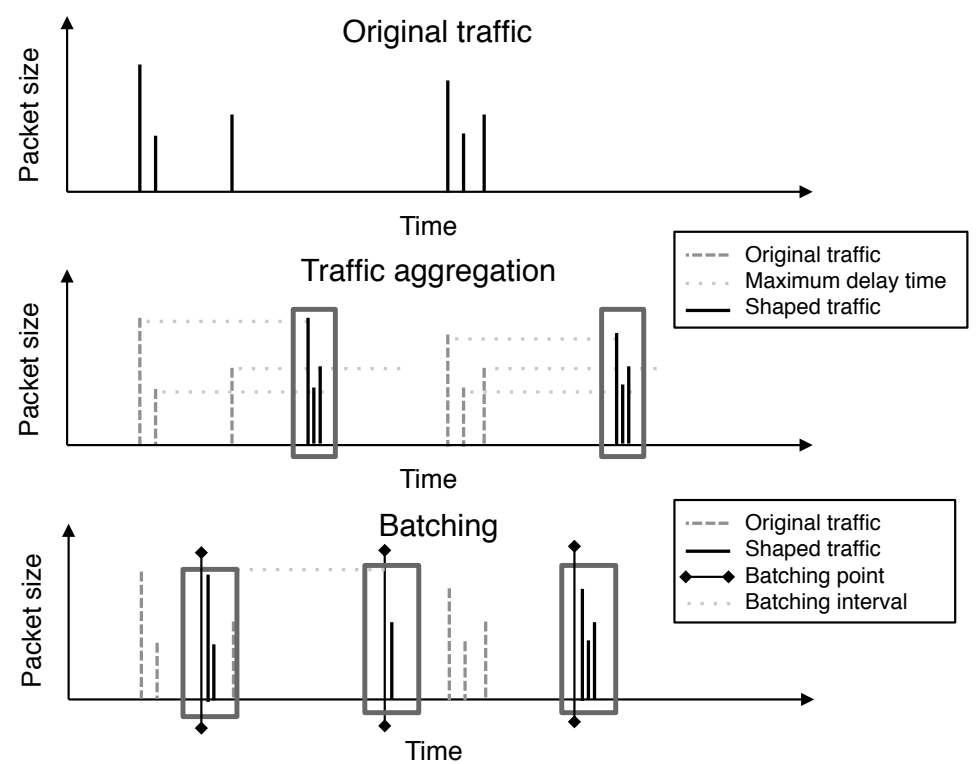

Figure 9.2: Traffic aggregation and batching.

- Traffic aggregation: TailEnder [24] employs an online scheduling algorithm using user-specified deadlines, and predicts future data accesses to prefetch data to reduce network transfers. Each packet is assigned a deadline and buffered. When the deadline of any of the packets is about to expire, all the buffer is sent in burst. CLBB (Chapter 5) schedules data transfers considering the inactivity timers and the RLC data buffers in combination. Our evaluation shows an improvement over TailEnder.

In a similar way to TailEnder, TailTheft [137] combines traffic aggregation and fast dormancy. Their work shows an improvement in prefetching the content by performing more accurate usage predictions.

Bartendr [223] schedules bulk data transmissions in periods of good signal strength. Nguyen et al. [163] improve the network condition predictions using a mixture of location, time and signal strength to schedule data transfers. Their work is orthogonal to ours since CLBB could benefit from their network estimations to alter the transmission based on the link quality.

Zhang et al. [277] propose different algorithms that aim at maximising 
the battery lifetime given a battery capacity and different workload considerations using a simplified model of the $3 \mathrm{G}$ interface. An optimal algorithm is proposed that transmits the maximum amount of data given the battery capacity. Their work does not consider the inactivity timers or RLC buffer thresholds that make the data pattern drastically impact the energy consumption.

At application level, the bundle technique proposed for IM applications in Chapter 7 follows the same idea of the above works by aggregating messages. While the above work attempt at reducing energy footprint for a generic class (or a subset of) application flows, this work explores tailor-made solutions for IM using application indicators and the user interaction knowledge obtained from our dataset.

Cinder [212] is an operating system provides to processes with a limited energy budget (reserve) and a energy discharge limits (taps). The high cost of sending data through the cellular interfaces leads to traffic aggregation since a single process has not enough energy budget to perform the transmission alone.

Coté et al. [55] propose an online packet scheduling based on packet deadlines in a similar way to TailEnder. The aim is to maximise the value of the packets sent and their analysis is based on competitive algorithms (online problem). However, only theoretical results are shown and the energy savings are not evaluated.

Based on the energy-delay tradeoff, the same concepts can be also applied to other technologies. For example, Palit et al. [171] employ packet aggregation applied to $\mathrm{WiFi}$, where packets are aggregated at the medium access control layer in order to fill a full frame and allow the device to extend its doze time. However, the timescale is in order of milliseconds whereas studies focusing on cellular communication consider seconds.

- Batching: Könönen et al. [123] propose the alignment of timers of different applications to perform synchronised bursty transmissions with a determined periodicity. Calder et al. [40] schedule data transmissions of applications with different transmission intervals in multiples of the shortest interval.

Siekkinen et al. [231] propose to shape the downlink streaming traffic using a proxy to create bursty transmissions. The maximum value of the inter-burst interval depends on the amount of data sent in the fast start and the TCP receive buffer size at the client. They analyse the energy savings of their approach for $3 \mathrm{G}$ using video streaming and audio streaming for LTE. Their results show that up to $60 \%$ savings are achievable when streaming music over LTE.

Huang et al. [107] show the energy consumption of the traffic generated when the screen of the device is off in $3 \mathrm{G}$ and $4 \mathrm{G}$ networks, and propose 
a combination of fast dormancy and batching to reduce background energy consumption.

- Piggybacking: Traffic backfilling [125] uses piggybacking to opportunistically transmit background data during unused gaps of energy tails between interactive traffic. This can be considered as opportunistic batching.

CLBB considers the current RRC state of the UE to schedule the background data. If background and foreground traffic differentiation is possible, the foreground traffic can alter the RRC state making CLBB to perform piggybacking, i.e., transmitting background data together with the foreground data. However our work does not present any evaluation in this regard.

Qian et al. [195] perform a simulation comparison of different approaches (fast dormancy, piggybacking, traffic aggregation and batching) showing that a combination of approaches can lead to higher savings.

Compared to the above works, CLBB is the only algorithm considering the RLC buffer thresholds when scheduling data transmissions to avoid unnecessary state transitions. Additionally our work was implemented as a kernel-level module in Android for evaluation.

\subsubsection{Link selection techniques}

Wake up techniques are the simplest version of link selection techniques, where a low power interface is used during idle time and a high power interface is woken up when there is a need for data communication $[3,179$, 229]. The general interface selection is more complex. It is based on the intuition that some interfaces (e.g., WiFi, 3G or Bluetooth) are more efficient than others given factors like power consumption of the interface, network conditions, link availability, amount of data sent or traffic pattern $[23,159$, 178,202]. These techniques aim at selecting the most energy-efficient link at a certain point in time considering different information. Measuring network availability is a common trend too $[205,261]$.

Switching between different interfaces is known as vertical handover and the IEEE 802.21 Media Independent Handover (MIH) [110,233] standard facilitates the handover initiation and handover preparation (network selection and interface activation). However, it does not standardise the handover execution mechanism and thus improvements have proposed [118,209]. In this context, Rahmati et al. [204] addresses the TCP/IP flow migration when performing a horizontal (i.e., same technology) or vertical handoff.

An interesting proposal is Intentional Networking [99], where developers provide semantic labels (e.g., small or large and foreground or background) to differentiate the data communication and match it to the different interfaces (WiFi or $3 \mathrm{G}$ ). Even though the aim of their work is on optimising the 
QoS rather than energy, the results of opportunistically deferring messages to send it via the selected interface can provide a benefit on the energy (similar to traffic adaptation techniques). However, their proposal requires developers to describe their transmissions via labels, and therefore rewrite parts of the applications.

\subsubsection{Interface power saving mechanisms}

Several works aim at improving the low-level power saving mechanisms provided by the standards by tuning parameters for different types of traffic. We categorise them by technology (cellular or WiFi).

Cellular interface: Recall that fast dormancy is the mechanism introduced in the 3GPP Release 8 that allows the UE to signal the desire to move to $\mathrm{PCH}$ before the expiration of the inactivity timers. Some proposals $[19,59,196]$ employ different flavours of fast dormancy to reduce the energy overhead of the inactivity timers. These works focus on $3 \mathrm{G}$ periodic transfers and background traffic. While current mobile devices use fixed fast dormancy timers to release the channel, the above works aim at mining application information to reduce energy while not increasing the signalling overhead and the state transitions (i.e., latency). Yang et al. [267] study the parameters of the power saving discontinuous reception (DRX) mechanism performed between the network and the mobile station.

Several works aim at optimising the values of the inactivity timers to reduce handsets' energy consumption while still maintaining network performance or reduce channel occupancy $[134,135,198]$. The different proposals range from global dynamic timers to fixed timers per user.

WiFi interface: Several works improve the standard power saving mechanisms of WiFi. The concept used in current devices (adaptive PSM) was introduced by Krashinsky et al. [124]. The sleep durations of the PSM are modified given the traffic levels while maintaining a level of RTT. Anand et al. [10] propose to adapt the PSM behaviour given the current traffic and the application intention provided by the developers. Pyles et al. [192] assign priority labels to each application using a machine learning classifier and the switch between PSM and CAM is triggered only by high priority applications. Using buffering techniques Bernardo et al. [30] propose an optimised power saving algorithm for continuous media applications.

While improving the power saving mechanisms is a generic technique, the communication of an inefficient application would still waste energy even with an optimised power saving mechanism.

\subsubsection{Network level techniques}

The previously presented works consider mostly a common resource perspective. This section describes works that adopt an individual resource perspective by considering several devices at the same time. These studies 
are complementary to our work. While the work in this area of research is vast, we present some works here since they achieve high energy savings.

Network level techniques combine different wireless interfaces and often employ device clustering techniques. The energy savings are mostly achieved by using a less energy consuming interface in a similar way to link selection techniques, but the focus of network level techniques is on creating the availability of the less energy consuming option by connecting among several mobile devices. Compared to the wireless sensor network literature [11, $38,98]$ where clustering has been deeply studied, mobile devices in cellular network lack a common goal and require fairness and incentives to cooperate. Moreover, the footprint characteristics of the different interfaces and the traffic load for the individual nodes is different and unpredictable.

The aim of the network level techniques is commonly divided in two cases: minimise the aggregated energy consumption of all the devices (global) or minimise locally the energy consumption of each terminal (local). Table 9.1 summarises main aspects of the complementary works categorised as network level techniques, which are further described below.

Several works employ clustering assuming that the mobile device users are interested in the same content $[7,182,266]$. Other applications include a cellular overload scenario [16], cellular traffic offloading and opportunistic device-to-device (D2D) data transmission [45, 93, 241]. Yoo and Park [269] consider Bluetooth clusters within WLANs.

Earlier work by the author, Watts2Share [251], proposes to form coalitions where the mobile devices employ a single cellular link offered by a member of the coalition (a rotating aggregator). The traffic of the coalition devices is consolidated in a single cellular link via a second wireless interface (e.g., WiFi or Bluetooth). Experimental studies based on real traffic traces and operator settings (using EnergyBox) show that the scheme provides high energy savings and reduces the signalling overhead. Our follow up work [33] generalises the scheme to organise the coalitions (proxy groups) and minimise the total energy cost as well as incorporating QoS considerations. The formation of the groups is formulated as an integer linear programming problem to minimise proxy changes while minimising the energy consumption as well as incorporating a reward-based sharing scheme for proxy rotation.

Mobile device clusters are often formed using WiFi software access points (SoftAP) or other technologies such as Bluetooth, and thus comparing the energy footprint of the different technologies used for D2D is important $[155,242]$. Improving the energy efficiency of the device that creates the SoftAPs has been studied by researchers $[41,94,117,228]$. Finally, methods to improve the performance of D2D communication (e.g., MAC layer [180]) have been also analysed [90,138].

While the energy saving potential of cooperation techniques is promising, security and privacy issues are commonly the main impediments that hold back the applicability of the techniques. The fairness between the different users and the incentives for the users to form part of the cooperation tech- 


\begin{tabular}{|c|c|c|c|c|c|c|c|c|c|c|c|c|c|}
\hline 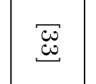 & 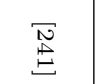 & $\begin{array}{l}\text { N } \\
\text { N }\end{array}$ & ت己 & $\begin{array}{l}\mathbb{N} \\
\mathbb{N} \\
\infty\end{array}$ & $\vec{\sigma}$ & 趈 & $\begin{array}{l}\text { N. } \\
0 \\
0\end{array}$ & 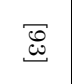 & 舁 & $\begin{array}{l}\bar{N} \\
\mathscr{\Omega} \\
\end{array}$ & $\begin{array}{l}\vec{\infty} \\
\stackrel{D}{N}\end{array}$ & $\begin{array}{l}\vec{\nabla} \\
\stackrel{\infty}{\infty}\end{array}$ & | \\
\hline 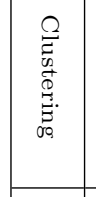 & 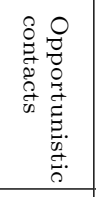 & 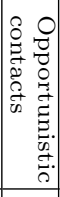 & 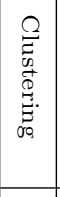 & 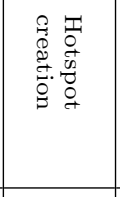 & 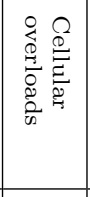 & 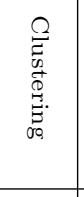 & 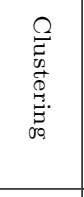 & 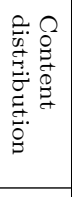 & 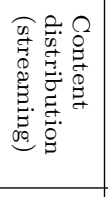 & 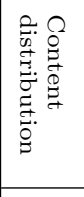 & 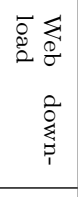 & 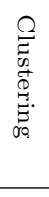 & 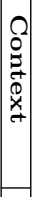 \\
\hline 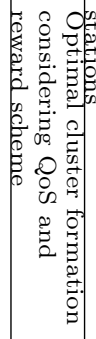 & 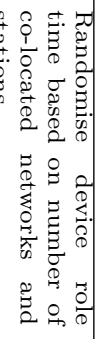 & 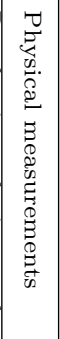 & 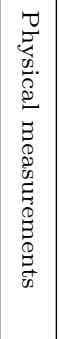 & 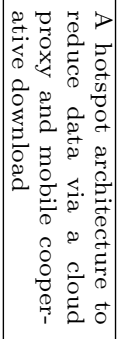 & 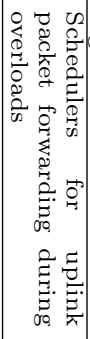 & 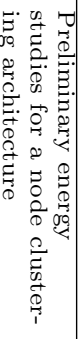 & 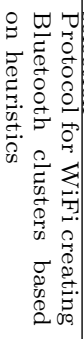 & 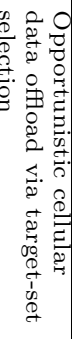 & 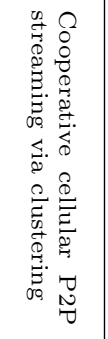 & 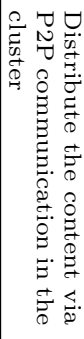 & 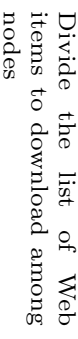 & 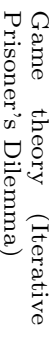 & $\begin{array}{l}0 \\
0 \\
0 \\
0 \\
0 \\
0 \\
0 \\
0\end{array}$ \\
\hline$\sum_{\substack{\Omega \\
\hdashline}}^{\infty}$ & 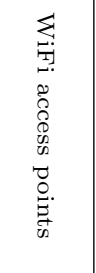 & 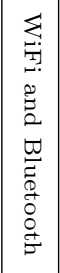 & 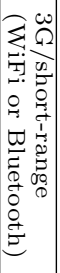 & $\sum_{\substack{\Omega \\
\hdashline}}^{\infty}$ & 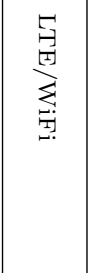 & $\sum_{\substack{\operatorname{Q} \\
\hdashline}}^{\infty}$ & 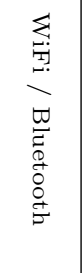 & 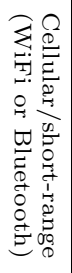 & 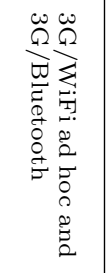 & 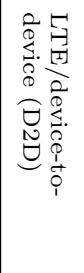 & 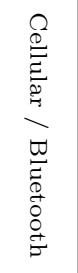 & 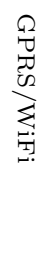 & \begin{tabular}{|c|}
0 \\
0 \\
0 \\
0 \\
0 \\
0 \\
0 \\
0 \\
0 \\
\hdashline
\end{tabular} \\
\hline 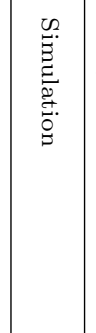 & 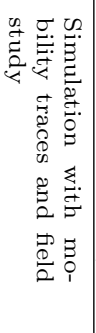 & 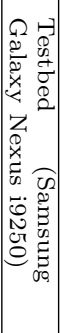 & 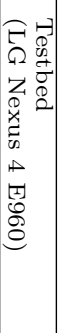 & 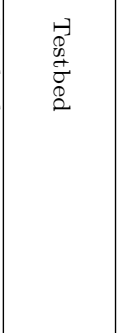 & 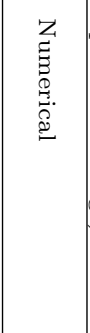 & 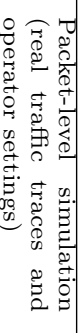 & 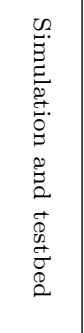 & 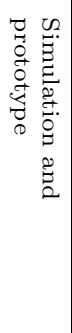 & 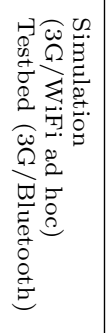 & 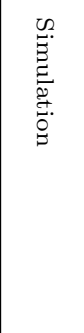 & 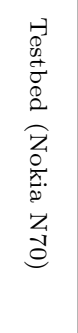 & 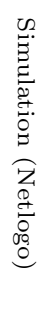 & 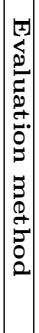 \\
\hline 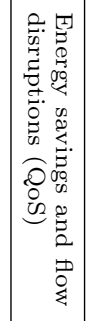 & 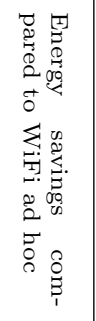 & 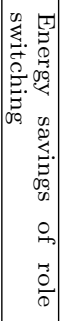 & 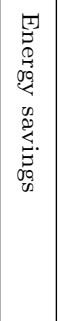 & 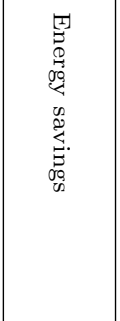 & 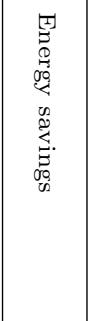 & 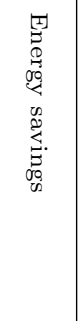 & 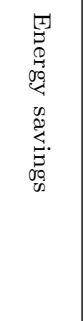 & 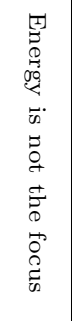 & 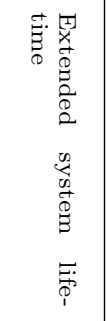 & 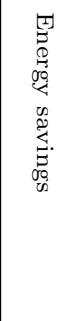 & 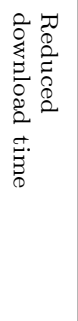 & 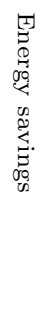 & $\mid \begin{array}{l}3 \\
0 \\
0 \\
\vdots \\
\vdots \\
\vdots \\
0\end{array}$ \\
\hline $\begin{array}{l}\dot{\partial} \\
\partial\end{array}$ & $\begin{array}{l}8 \\
\stackrel{8}{\partial 9}\end{array}$ & 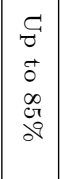 & 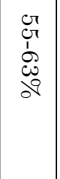 & 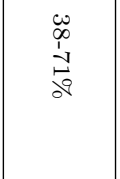 & 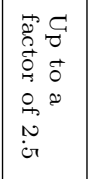 & 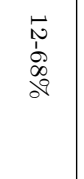 & 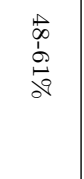 & بٌ & 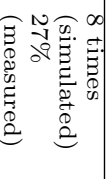 & $\begin{array}{l}\text { P } \\
\text { dn } \\
\text { dQ } \\
\text { d }\end{array}$ & $\stackrel{\vec{d}}{\vec{d}}$ & $\begin{array}{l}\text { gr } \\
\text { dq }\end{array}$ & $\mid \begin{array}{l}\mid 70 \\
0 \\
0 \\
2\end{array}$ \\
\hline
\end{tabular}


niques are also significant problems to solve, which require the application of appropriate energy apportionment policies.

The increasing number of works considering energy consumption as a vital issue within mobile communication (and in general) shows the importance of an area with a very active community. 
"Esana esan, emana eman."

What's said is said,

what's given is given.

Basque proverb

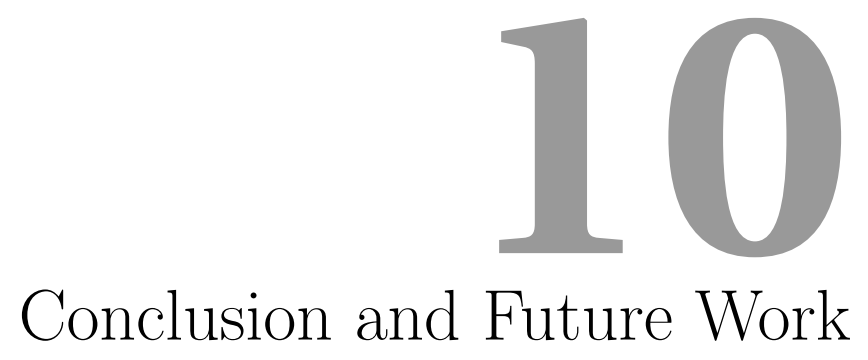

This chapter presents the general conclusions based on the performed work. We also discuss the potential future directions.

\subsection{Conclusions}

Energy is a resource of paramount importance in computer and communication systems. This is especially true for battery powered devices where it is a scarce and essential resource, which calls for its optimal utilisation. Analysing the energy footprint characteristics of individual system components and entities is a precondition for energy efficiency. There is a need to reveal hidden inefficiencies and promote low energy footprint designs and solutions.

The main context of our work is mobile communication at the device end, and thus we link the conclusions to the original problem formulation points presented in section 1.4:

\section{Energy awareness}

"Provide efficient means to support the analysis of communication energy footprint at the user end. The proposed approach should complement physical power measurements, and facilitate the design of energy-efficient solutions."

Throughout the thesis we argued that improving energy efficiency starts by being able to quantify energy consumption. We identified the data pattern as one of the main aspects impacting the energy consumption of the user 
end, and proposed EnergyBox in order to provide energy awareness. It allows us to estimate the system energy function $E$ for mobile communication. In addition, we illustrated how the tool facilitates the process of revealing energy-inefficient data patterns by quantifying the energy consumption of popular mobile applications in isolation. EnergyBox has already been used in research (e.g., throughout the thesis), other research projects [91, 164] and has been integrated in the testing environment of the online music service Spotify [87]. This shows its practicality and utility to provide energy awareness and complement physical power measurements.

While EnergyBox certainly supports the analysis of communication energy, the measurement effort required to integrate network and mobile device input values into EnergyBox should not be overlooked. Even though mobile operators are not likely to reveal their network configuration parameters, we provided algorithms to estimate network parameters as well as methods to measure the power consumption of devices which partially solve the problem. However, given the variety of mobile networks and vendors, determining which parameters can be representative as well as keeping the models up to date are interesting aspects that remain relevant.

Using EnergyBox we quantified the energy of applications in isolation and pinpointed improvements to reduce their consumption. We characterised the data pattern using indicators such as inter-packet interval or packet size and compared the energy consumption of similar applications. Nevertheless, determining the energy efficiency of single application or feature requires also the characterisation of aspects like the utility of the application for the user given the consumed energy.

Finally, it is worth mentioning that the general methodology used to model energy consumption of wireless interfaces is applicable to other systems within and outside mobile communication (e.g., base stations or other devices). In addition, EnergyBox (or similar approaches) could be used to analyse the impact of configuration decisions on actual data patterns.

\section{Methods to support energy management}

"Identify and provide methods to perform energy accounting to ultimately support energy management. The proposed means should determine the contribution of the entities in the system to the total energy consumption in order to evaluate their energy efficiency and be illustrated in the context of networking applications."

While analysing the entities in isolation is certainly beneficial, a system perspective is required to examine the actual efficiency of the entities in the system and provide transparency. Leveraging cooperative game theory we analysed different methods to determine the contribution of the entities to the total energy consumption, which is essential in energy accounting in order to support energy management. Even though there is no ground truth 
for the energy apportionment problem, we showed that selecting a policy depends on the trade-off between fairness and the other evaluation criteria (i.e., computation complexity and information requirement). We provided guidelines to select an energy apportionment policy depending on the purpose of the apportionment and the energy consumption characteristics of the system.

Selecting an energy apportionment policy implies the selection of fairness properties. A common approach has been to select the apportionment policy first without considering the potential fairness implications (see Chapter 8 and section 9.3). Instead, we believe that analysing the desired fairness properties for the system is the first step to further select an appropriate apportionment policy. In this context we identified 5 relevant fairness properties for energy apportionment.

The recommended energy apportionment policies require energy information as input. The combination of an appropriate energy apportionment policy and EnergyBox can identify the efficiency of the entities in isolation as well as in the system in the context of mobile communication. By analysing the energy consumption of isolated groups of entities we can evaluate the efficiency of their interactions. This can lead to better ways to coordinate applications' transmissions.

We show that our efforts in quantifying the energy consumption in isolation are valuable since the most suitable apportionment policies employ the isolation energy to determine an entity's contribution. Moreover, the choice of energy apportionment policy is tightly coupled to providing incentives to reduce the total energy consumption of the system. An appropriate policy can provide incentives by rewarding the efficient entities or penalising the inefficient ones as well as the developers. Informing the end user is also a relevant application of energy apportionment.

While there are still many open problems, some of the proposed solutions have a great potential at multiple levels in mobile communication as well as in other contexts. For example, an appropriate energy apportionment policy enables evaluating the efficiency of applications in a dynamic system in addition to in isolation, or identifying the most consuming features and processes of an application under their actual usage (e.g., once the application is released for testing). Analysing the energy of the mobile infrastructure (e.g., small cells) and the impact of mobile devices and their communication is an interesting application.

\section{Energy saving techniques}

"Explore and develop methods to reduce the energy consumption of the system. The proposed methods should help to achieve energy efficiency for the entities in the system given knowledge about the activities. The aim is to gain an understanding of the advantages and disadvantages of tailor-made and traffic class based solutions." 
Once the methods to quantify and understand the factors affecting energy consumption are established, it is feasible to devise means to control and reduce energy consumption. In the search for energy-efficient solutions we considered the isolated entity and the system perspectives:

- Entity perspective: It is often the case that entities can maximally influence their own consumption in isolation, but they have small or no control over the interactions with other entities when using the components of the system. In this respect we argued that developers can boost the energy efficiency of their applications in isolation with the support of appropriate tools such as EnergyBox. We showed that quantifying the energy of different design choices, considering application usage patterns and evaluating new features can lead to a reduced footprint. In addition, we designed tailor-made solutions informed by user utilisation information to provide a trade-off between message delivery delay and energy consumption.

We analysed the energy of the extra features (e.g., typing notification) in isolation (with and without the feature). An alternative to this approach could be to employ an energy apportionment policy to determine the actual contribution of the feature to the total application consumption. This would require to identify or tag the traffic of the feature and differentiate it from the rest of traffic of the application. However, it would enable the option to analyse the energy consumption outside the testing environment.

- System perspective: System level solutions operate under the application layer and can influence application interactions to promote an efficient coordination. However, their influence over applications' operation and thus their individual energy consumption is somewhat limited.

The advantage of the developed background traffic class solution is the capability of considering the traffic from several applications at once. Traffic class solutions can enforce energy-efficient communication for several applications. Moreover, a system level approach like ours can be informed by the energy footprint characteristics of the system (e.g., the radio layer information), which can result in more efficient solutions.

One of the main remaining challenges is to understand how to provide the means to use the energy-efficient solution from the application perspective. We pinpointed that traffic class solutions require application traffic differentiation, and thus application cooperation becomes important to fully benefit from the potential energy savings. In this respect energy apportionment policies and energy management can promote the adoption of system-level energy-efficient solutions by rewarding the applications that make use of them. 
We conclude that methods focusing on both entity and system perspective are complementary and required in order to maximise the total efficiency of the system. While tailor-made solutions take advantage of a richer knowledge of the application layer and can maximise entities' energy efficiency in isolation, without any system level coordination chances are that the aggregate entity operation will not be fully optimised. Yet, given that the entities are optimised for lower energy usage, the aggregated energy consumption may be lower than running inefficient entities. Traffic-class based solutions can coordinate application traffic, but the solutions are required to provide effortless and simple methods to the application layer in order to achieve entity cooperation and be successfully employed.

Finally, an important aspect that we did not consider in this work is the control of the provided energy-efficient solutions. While we created the trade-off between energy and delay, we did not provide any method to select the parameters of the solutions. There is a need to quantify the perceived functionality benefit from the user side and provide simple but yet effective means to the user to influence the energy consumption of the system and applications. Thus, the user, application and operating system interplay is interesting to explore in order to optimise resource usage while maximising the user experience.

To sum up, this thesis has contributed towards making energy consumption a first class system citizen. We hope that the current and future research efforts will be followed by both application and system software developers by adopting the proposed new methods, tools and solutions to improve energy efficiency.

\subsection{Future work}

Energy efficiency is a very active and a large area of research. While our work focused only on certain areas, there are many other research directions. We list some interesting future directions, ranging from immediate work based on the presented studies to more general future work. These directions are categorised into future work related to the main context of study of the thesis (i.e., mobile communication) and directions considering general problems related to energy consumption which are applicable to a multitude of contexts. The following directions consider energy consumption in mobile communication:

- Evaluating energy-efficiency: As previously mentioned, the development of methodologies and metrics to evaluate the efficiency of a single application still remain as an open question. How can we characterise the utility of an application? How can we define energy efficiency classes? Which usage scenarios are representative for the evaluation? 
In addition, while tailor-made solutions are a feasible approach for developers, the time spent to optimise the energy usage of their application can be detrimental to the time to market and other priorities (e.g., bug fixes or development of new functionalities). Thus, in a more technological aspect, automatically evaluating energy as part of the testing environment is a first step. However, instead of trying to mimic all relevant test cases, studying energy consumption once the application is deployed becomes highly interesting.

- Incentives to developers: Even though the combination of energy apportionment policies and EnergyBox (or similar) can facilitate the analysis of energy consumption and reveal the most consuming entities, none of these two approaches impose any responsibility on developers to improve the energy efficiency of their software. Thus, we believe that there is still a need to provide incentives to developers to adopt energy-efficient solutions and optimise the energy consumption of their applications.

These incentives can come from different actors in the ecosystem, since energy efficiency can benefit mobile vendors, end users and even cellular operators. For example, a simple comparison of software providing similar functionality and ranking them by the consumed energy (e.g., similar to the EnergyStar initiative ${ }^{1}$ ) could trigger the need to optimise software efficiency for the most consuming applications. These type of studies can be part of marketing to advertise applications.

- Tailor-made user-centric solutions: We showed that tailor-made solutions can be informed by user information. For example, user activity (e.g., the screen is off, the user switched to another application) and context information (e.g., built-in sensors) are interesting inputs. However, we believe that applications could provide energy management settings to the user in the same way that they provide performance settings (e.g., increase the video quality). Providing the option to enable or disable certain features can be an approach with an easy implementation.

- Energy ranking of reusable modules: We argued that developers should quantify the energy consumption of different design options and illustrated the benefit in the context of IM applications and location sharing services. However, as previously mentioned, the agenda of the developers can show other priorities rather than optimising energy consumption.

A previous work proposed to label the energy consumption of applications to help the users select the most efficient one among similar applications [260]. In a similar way, some of the most common software module alternatives (e.g., libraries or protocol implementations)

\footnotetext{
${ }^{1}$ https://www.energystar.gov
} 
could be quantified in terms of energy and ranked accordingly, which would assist developers in the selection between modules providing similar functionalities.

- Towards generic traffic class solutions: Our work treated a single traffic class separately. The coordination of the different traffic classes generated from different applications is also challenging due to the different QoS requirements. Traffic differentiation and application coordination becomes the main limitation of the traffic class approach. Application level traffic differentiation would require the developers to cooperate by labelling their transmissions. Lower layer traffic differentiation without application cooperation is an interesting approach to investigate. Future traffic estimation per-application can be a significant aid to optimise the scheduling of data transmissions.

- Individual and common resource perspective: A great part of work presented in this thesis adopted a common resource perspective of mobile devices. Our complementary studies in the area of network level techniques $[33,251]$ adopt an individual resource perspective to reduce the energy consumption of groups of mobile devices using data relaying.

A combination and coordination of the individual and common resource perspective can further improve the energy efficiency in this context. Additionally, employing an appropriate energy apportionment policy at network and device level can reveal inefficiencies and provide incentives for cooperation and optimisation at both levels.

- Holistic infrastructure and user terminal approaches: We focused on the mobile device side in mobile communication, and while the research in green networking focuses either on the infrastructure or the user side, approaches considering the cooperation of both ends could lead to more efficient solutions. For example, user transmissions can be scheduled to enable sleeping techniques for infrastructure nodes and vice versa. Studying the interplay between the infrastructure and devices (e.g., mobile or sensor devices) in terms of energy is a potential future work. We hope that energy consumption and sustainability become an important aspect in the developing 5G standard. Energy apportionment becomes highly relevant in this context.

The following future directions consider the general problem of energy consumption and could benefit different contexts (included mobile communication):

- Energy apportionment: A direct extension to our energy apportionment study includes applying its insights in systems from different contexts and empirically studying the impact of the incentives on energy efficiency. Extending the study with additional properties (e.g., 
reduced game property, monotonicity or additivity) or other cost allocation methods (e.g., $\tau$-value or cost gap method) is certainly interesting.

Developing means to measure or better approximate the system function for different systems is a key requirement which can provide insights about the most suitable policy to use given the structure of the system function.

An interesting aspect to study is the impact of using different energy apportionment policies in the same context. For example, application developers might use a certain policy for profiling while the operating systems employ another policy to provide incentives. Are we limited to select a single policy per system? What is the impact of the coexistence of different policies?

- User-centric energy management: We believe that the user should have the final say when it comes to managing the energy consumption in user-centric systems. Energy apportionment can provide the required transparency in the system, but we need to provide methods to increase the influence of the user in energy management while still providing simple and intuitive information and control.

- Energy as part of QoS: As we mentioned in the area of mobile communication, there is a need to focus efforts on developing methods and metrics for making energy consumption a parameter in QoS. Different QoS classes can be defined based on all the parameters including energy which would allow to define energy requirements for entities as well as means to allocate energy resources.

- Unify methods from different fields: While energy consumption is often considered system dependent, we believe that there is enough common ground among the different fields studying energy efficiency to look for common methods and solutions. The energy apportionment problem is an example, which has been studied in different contexts ranging from wireless sensor nodes to building environments or data centres. We believe that the progress made in some contexts focusing on specific systems can absolutely benefit other contexts.

While the potential directions can significantly help to reduce the energy consumption in mobile communication as well as in other contexts, there is still plenty of work to be done to achieve energy-efficient systems, software and communication. 
Appendices 



\section{A Energy for Recharging Smartphones}

This Appendix estimates the amount of energy used worldwide to power up the current number of smartphones.

We selected two common smartphones (iPhone 5 and Samsung Galaxy S3) and obtain its time to charge from $0 \%$ to $100 \%$, and the average power drain during the recharge period. The iPhone 5 and Samsung Galaxy S3 are shipped with $1440 \mathrm{mAh}$ and $2100 \mathrm{mAh}$ batteries respectively. The Galaxy S3 consumed 12.3 Wh, whereas the iPhone 5 consumed $9.5 \mathrm{Wh}^{1}$. Note that the tendency in the manufacturers is to increase the battery capacity, where the recharging time will increase.

This estimation uses a simplifying assumption that an average smartphone user charges her device once per day. The year consumption is obtained by multiplying the consumption by 365 days: $4.49 \mathrm{kWh}$ and $3.47 \mathrm{kWh}$ for the Galaxy S3 and iPhone 5 respectively. For simplicity, we consider 4 $\mathrm{kWh}$ as the year consumption for every smartphone (10.95 Wh per day). Given a billion of smartphones shipped around the world in $2013^{2}$ and 4 $\mathrm{kWh}$ year consumption, the total cost of recharging the global smartphones becomes 4 TWh per year.

The capacity of production of a nuclear power plant varies much depending on factors such as the number of reactors. $2346 \mathrm{TWh}$ of nuclear electricity production were registered in $2012^{3}$. Fig. A.1 shows the year electricity production of the 437 operational nuclear power plants in the world ${ }^{4}$. The cost of charging the smartphones corresponds to a relatively small power plant.

The average annual electricity consumption for a U.S. residential utility customer was $11280 \mathrm{kWh}$ in $2011^{5}$. Therefore, the energy spent by charging 


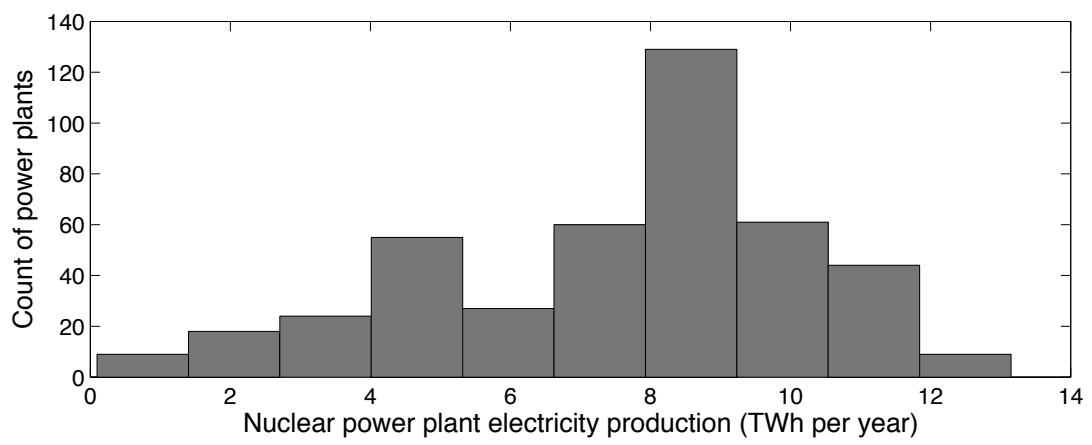

Figure A.1: Energy production of the world's nuclear power plants.

the smartphones is equivalent to the energy consumption of 354609 households (4 TWh / $11280 \mathrm{kWh}$ ) or the energy production of a small nuclear power plant.

\footnotetext{
${ }^{1}$ http://blog.opower.com/2012/09/how-much-does-it-cost-to-charge-an-iphone-5-athought-provokingly-modest-0-41year/

${ }^{2}$ http://www.gartner.com/newsroom/id/2227215

${ }^{3}$ http://www.world-nuclear.org/info/Facts-and-Figures/Nuclear-generation-bycountry/\#.Ua9NHHC3i84

${ }^{4}$ http://world-nuclear.org/nucleardatabase/rdResults.aspx?id=27569 Retrieved 2013/05/05

${ }^{5} \mathrm{http}: / /$ www.eia.gov/tools/faqs/faq.cfm?id $=97 \& \mathrm{t}=3$
} 


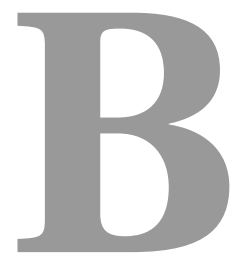

Measurement setup

Fig. B.1 shows an overview of our measurement setup used for $3 \mathrm{G}$ and WiFi measurements. The setup is described separately for $3 \mathrm{G}$ and WiFi.

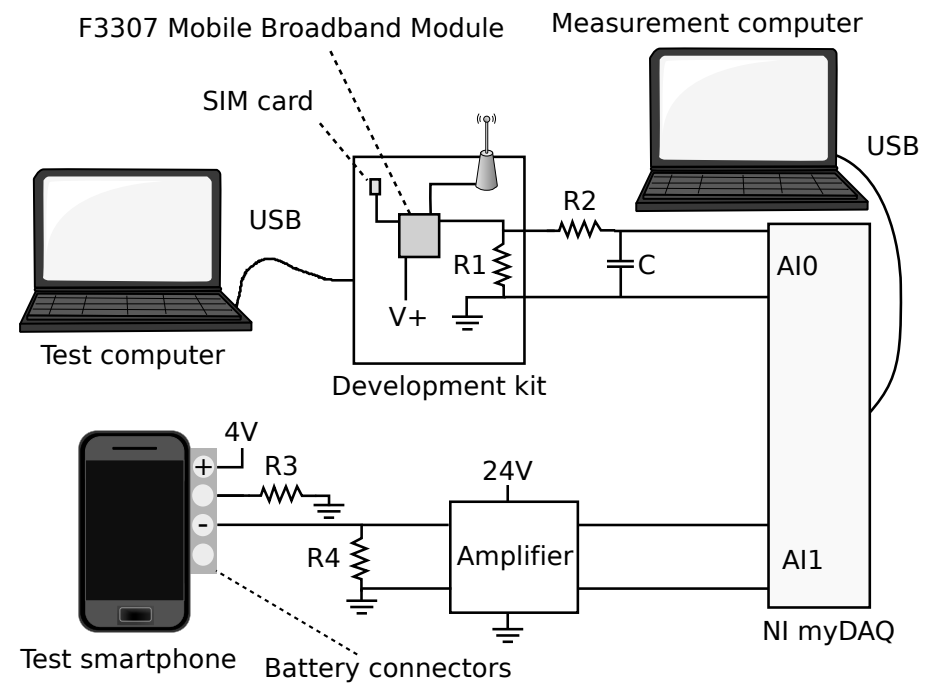

Figure B.1: Measurement setup for 3G and WiFi.

3G setup: The 3G measurements were performed on a power-efficient mobile broadband module (Ericsson F3307) provided by Ericsson AB (KRY 901214/01, marked as Development Kit in Figure B.1). Since this exposes 
interfaces to measure the power consumption of the $3 \mathrm{G}$ modem easily isolating it from the rest of system (e.g., CPU or screen), we employ this platform instead of the $3 \mathrm{G}$ module in a smartphone. The mobile broadband module provides $2 \mathrm{G}, 3 \mathrm{G}$ and GPS connectivity. It is designed to provide mobile broadband to consumer electronic devices such as tablets and laptops. The module provides uplink and downlink speeds up to 5.76 and 7.2 Mbps respectively implementing the High Speed Packet Access (HSPA) standard.

The measurement setup depicted in Figure B.1 consists of the mobile broadband module placed on an Ericcson's Developer Starter Kit. The kit provides network connectivity via USB to a test computer that runs a Ubuntu 10.10 with a firewall activated in order to allow only the desired network connections. The measurements were performed using a National Instruments myDAQ data acquisition device sampling the voltage drop over a shunt resistor $(\mathrm{R} 1=0.1 \Omega$ in Fig. B.1) at $1 \mathrm{kHz}$. The power consumption is derived from the voltage and in order to avoid any anti-aliasing effects a low-pass filter of approximately $16 \mathrm{~Hz}(\mathrm{R} 2=10 \Omega \mathrm{k}$ and $\mathrm{C}=1 \mathrm{uF}$ in the figure) was added.

Most measurements were performed using a Subscriber Identity Module (SIM) card from the network operator TeliaSonera providing full access to the available capacity of the Sweden 3G network. Unless specified, all the measurements were performed in the same location at university where the received signal strength did not vary significantly.

WiFi setup: The WiFi measurements were performed removing the battery of the smartphones under test and intercepting the battery terminals of the smartphone using copper tape. We add a low-side sensing circuit with a shunt resistor $(\mathrm{R} 4=0.1 \Omega)$. The voltage drop over the shunt is amplified using the Phoenix Contacts NI-MCR-SL-SHUNT-UI-NC ${ }^{1}$ isolating amplifier (maximum transmission error of $0.4 \%$ and gain 50 ). The power consumption is sampled at $1 \mathrm{kHz}$ using the myDAQ and the measurement values where compared against a digital multimeter (Agilent U1252B) for validation purposes.

Apart from $\mathrm{V}+$ and ground pins, smartphone batteries often have a temperature and a communication line back to the phone. These pins are used to tell the phone the charge condition of the battery (smart battery interface) and when the charge cycle is complete. We added R3 (33 k $\Omega$ ) in order to allow smartphones to switch on.

Ideally, one would like to only measure the communication energy (i.e., the energy spent by the peripheral hardware). We adopt a methodology to isolate the communication energy from the rest of the system as in earlier works [208]. All smartphone components are switched off (e.g., screen, sensors or other radios). Since the operating system is preemptively multitasking, different processes are waking up and consuming resources such as CPU. An example of this effect is shown in Figure B.2, where it is hard to

\footnotetext{
${ }^{1}$ http://www.conrad.com/ce/en/product/569243/Isolating-amplifier-MINI-MCR-SLSHUNT-UI-NC-2810780-Phoenix-Contact
} 
separate the energy consumed for the network transmission from the aggregated power consumption.

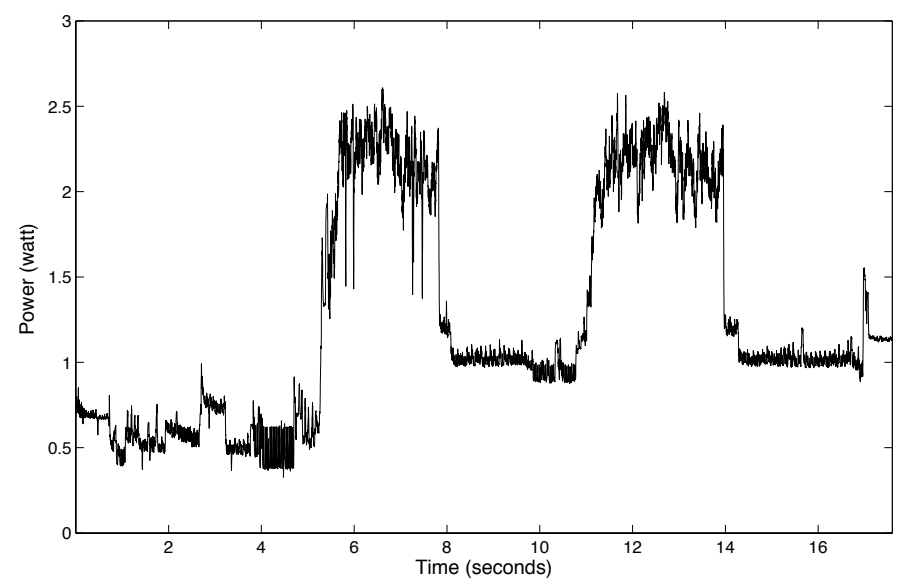

Figure B.2: Aggregated power consumption for CPU and network transmission.

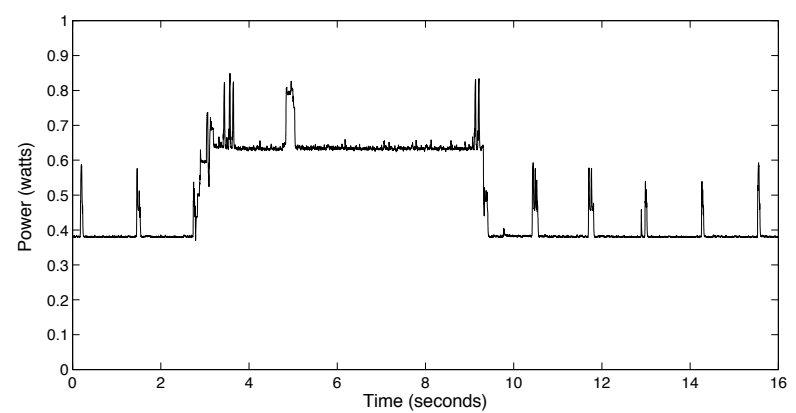

Figure B.3: Stabilised CPU power trace and a network transmission.

In order to stabilise the power trace and isolate the transmissions from the rest of the system, we fix the CPU frequency and run a low-priority background process in a busy-loop. This stabilises the power trace. Figure B.3 shows the aggregated energy of a network transmission and the CPU with our methodology. The CPU creates an almost constant power consumption $(0.37 \mathrm{~W})$ that enables the isolation of the communication energy.

The drawback of this technique is that we cannot distinguish between the CPU load created by the test and the background load. However, this should have little effect at the time of studying the peripheral hardware of the phone such as the wireless interfaces. 


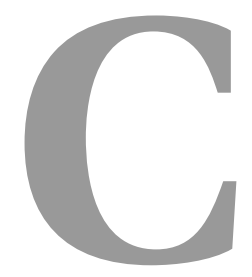

\section{Baseline Energy Footprint for IM Apps}

A qualitative comparison of our IM application and the popular IM applications is shown in this appendix. We are interested on knowing the energy consumption of the most basic 1-to-1 chatting functionality, thus our prototype is expected to have a lower energy consumption. The Short conversation is used as example and the test is repeated 5 times.

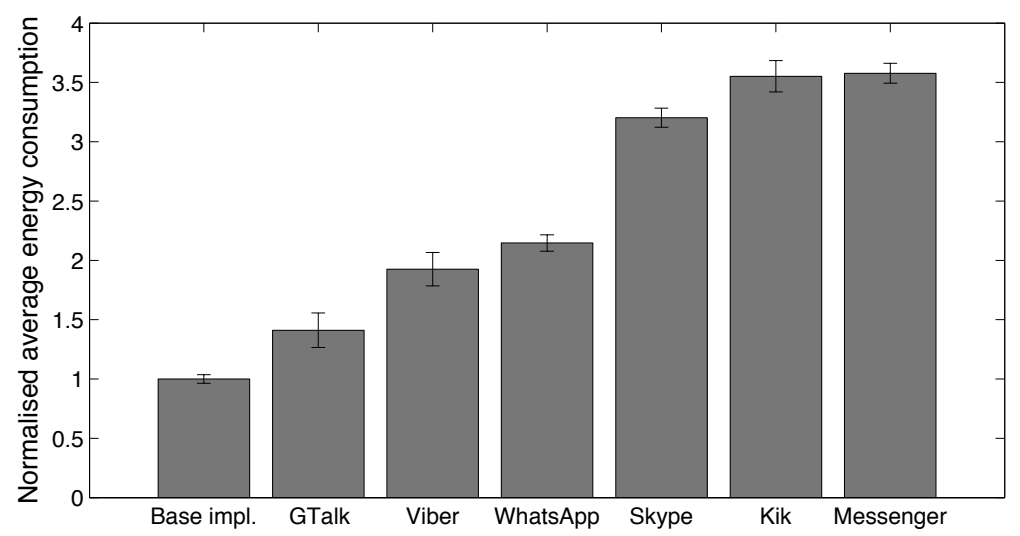

Figure C.1: Energy consumption of the basic implementation compared to popular IM applications when sending the Short conversation.

Fig. C.1 shows the energy consumption of the different applications normalised to the base case (22.56 joules). The energy cost of only sending the Short conversation increases $40 \%$ by sending it with GTalk instead of with the base implementation. Since applications are black boxes, no conclusions 
can be drawn from this result. However, it provides insight of how much room there is for implementing extra features before the base implementation reacher the energy consumption of other applications. The energy consumption of Messenger seems unjustifiable based on the fact that it uses the same protocol.

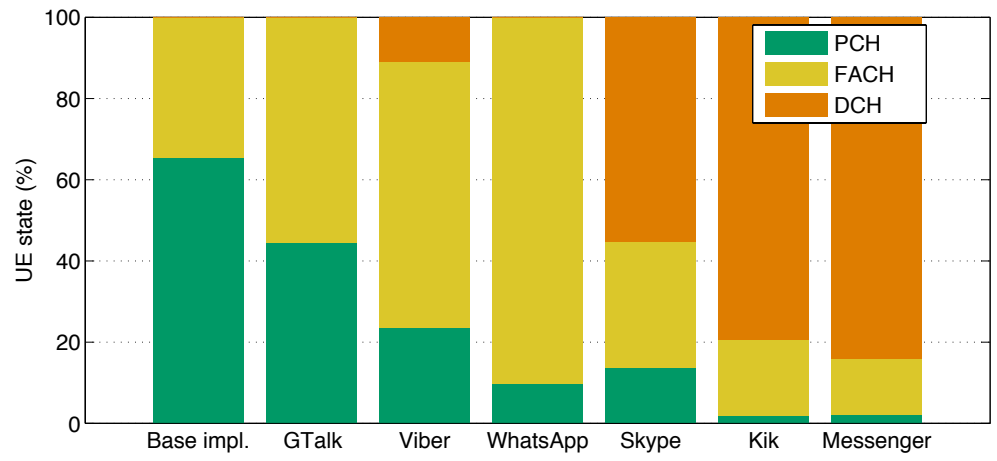

Figure C.2: Time spent in the different RRC states of the basic implementation compared to popular IM applications when sending the Short conversation.

Fig. C.2 shows the time spent by the client in the different RRC states for a single run of the test. The least consuming applications make a more efficient use of the FACH channel by sending small packets. The main difference between the base implementation and GTalk is the amount of data sent, which makes the device to spend more time in the FACH state. Even though Viber switches the UE to DCH for a short period, it still consumes less than WhatsApp since the time spent in the PCH state is longer. The data sent by GTalk and WhatsApp made the UE to occasionally switch to DCH for short time (3 out of 5 tests for GTalk and 1 out of 5 for WhatsApp). However, efficiently using the DCH channel by sending data in bursts and keeping the UE in PCH for longer periods (e.g., GTalk and Viber) can be less consuming than continuos data transfer in FACH (e.g., WhatsApp). Data intensive IM applications that send big packets (e.g., Skype, Kik and Messenger) make the device spends most of the test time in the DCH state, consuming a large amount of energy. An energy-efficient IM application should avoid the DCH state when possible since the data to be sent is generally small. 


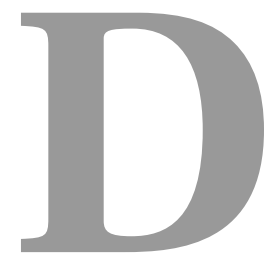

\section{Fairness Property Proofs}

This appendix provides our proofs regarding the symmetry, dummy player, individual rationality, coalitional rationality and entity desirability properties shown in Table 8.2. We also provide a reference to the properties that we do not analyse in this appendix.

Table D.1 provides the reference to the works where the other properties are studied. The table also refers to the theorems in this appendix.With the exception of entity desirability, the properties of the game-theoretical policies (Policies 5, 6 and 7) are well known results in the game theory community, and thus we provide some references as examples. Note that the references of the coalitional rationality property refer to the individual rationality theorems since a policy does not satisfy coalitional rationality if it does not satisfy individual rationality.

Table D.1: Reference to the fairness properties results for each policy.

\begin{tabular}{|c|c|c|c|c|c|}
\hline Nr. & S & DP & IR & CR & ED \\
\hline 1 & {$[250]$} & {$[86]$} & {$[86]$} & Not IR & 23 \\
\hline 2 & 1 & 6 & 16 & 16 & 24 \\
\hline 3 & 2 & 7 & 17 & 17 & 25 \\
\hline 4 & {$[250]$} & {$[86]$} & 18 & 22 & 26 \\
\hline 5 & {$[226]$} & {$[226]$} & {$[147$, p. 151$]$} & {$[129$, sec. 5] } & {$[211$, p. 42] } \\
\hline 6 & {$[220]$} & {$[220]$} & {$[220]$} & {$[220]$} & {$[211$, p. 151] } \\
\hline 7 & {$[214]$} & {$[214]$} & {$[214]$} & {$[214]$} & {$[214]$} \\
\hline 8 & 3 & 8 & 19 & 19 & 27 \\
\hline 9 & 4 & 9 & 20 & 20 & 28 \\
\hline 10 & 5 & 10 & 21 & 21 & 29 \\
\hline
\end{tabular}

S: Symmetry. DP: Dummy player. IR: Individual rationality.

CR: Coalitional rationality. ED: Entity desirability. 


\section{D.1 Symmetry}

This section presents the proofs regarding symmetry: Policies 2 and 3 satisfy the property, whereas Policy 8, 9 and 10 do not satisfy it. The symmetry property presented in section 8.4.1 is the following: if $E(S \cup\{i\})=E(S \cup\{j\})$ for all $S \subseteq N \backslash\{i, j\}$, then $\pi_{i}=\pi_{j}$.

Theorem 1. Policy 2 (Proportional to isolation) always satisfies symmetry.

Proof. In order to prove symmetry will assume $E(S \cup\{i\})=E(S \cup\{j\})$ for all $S \subseteq N \backslash\{i, j\}$, and prove that $\pi_{i}=\pi_{j}$ for Policy 2. From Equation 8.4 the apportionment for entities $i$ and $j$ is the following:

$$
\begin{aligned}
& \pi_{i}=E(\{i\}) \frac{E(N)}{\sum_{k \in N} E(\{k\})} \\
& \pi_{j}=E(\{j\}) \frac{E(N)}{\sum_{k \in N} E(\{k\})}
\end{aligned}
$$

It follows from the assumption that $E(\{i\})=E(\{j\})$ for $S=\emptyset$. Hence, from Equation 8.4 and $E(\{i\})=E(\{j\})$ we have that:

$$
\pi_{i}=E(\{i\}) \frac{E(N)}{\sum_{k \in N} E(\{k\})}=E(\{j\}) \frac{E(N)}{\sum_{k \in N} E(\{k\})}=\pi_{j}
$$

Policy 2 always satisfies symmetry.

Theorem 2. Policy 3 (Marginal contribution) always satisfies symmetry.

Proof. In the proof we will assume $E(S \cup\{i\})=E(S \cup\{j\})$ for all $S \subseteq$ $N \backslash\{i, j\}$, and prove $\pi_{i}=\pi_{j}$ for Policy 3 .

We know that $E(S \cup\{i\})=E(N \backslash\{j\})$ and $E(S \cup\{j\})=E(N \backslash\{i\})$ for $S=N \backslash\{i, j\}$. Thus, it follow from the assumption that $E(N \backslash\{i\})=$ $E(N \backslash\{j\})$.

From Equation 8.5 and $E(N \backslash\{i\})=E(N \backslash\{j\})$ we have the following:

$$
\begin{aligned}
& \pi_{i}=E(N \backslash\{i\}) \frac{E(N)}{\sum_{k \in N}[E(N)-E(N \backslash\{k\})]} \\
= & E(N \backslash\{j\}) \frac{E(N)}{\sum_{k \in N}[E(N)-E(N \backslash\{k\})]}=\pi_{j}
\end{aligned}
$$

Policy 3 satisfies symmetry.

Since activity and energy are not necessarily correlated we can easily find a counterexample for the surrogate-based policies.

Theorem 3. Policy 8 (Proportional to activity) does not always satisfy symmetry.

Proof. A counterexample is enough to prove the theorem. We show that $E(S \cup\{i\})=E(S \cup\{j\})$ for all $S \subseteq N \backslash\{i, j\}$ holds in the counterexample and prove that $\pi_{i} \neq \pi_{j}$ for Policy 8 . 
Table D.2: Two entity game with symmetric entities.

\begin{tabular}{|c||c|c|c|}
\hline$S$ & $\{1\}$ & $\{2\}$ & $\{1,2\}$ \\
\hline$E(S)$ & 2 & 2 & 3 \\
\hline \hline$a(S)$ & 4 & 5 & 7 \\
\hline
\end{tabular}

Table D.2 shows a system $N=\{1,2\}$ together with the energy consumption and activity function $a(S)$ for all possible configurations of this system.

Since $E(\{1\})=E(\{2\})$ for $S=\emptyset$ the antecedent for the symmetry requirement holds.

From Equation 8.10 and the values in Table D.2 the apportionment for entities 1 and 2 is computed: $\pi=(1.33,1.66)$. Since $\pi_{1} \neq \pi_{2}$, Policy 8 does not satisfy symmetry.

Theorem 4. Policy 9 (Time slicing) does not always satisfy symmetry.

Proof. A counterexample is enough to prove the theorem. We show that $E(S \cup\{i\})=E(S \cup\{j\})$ for all $S \subseteq N \backslash\{i, j\}$ holds in the counterexample and prove that $\pi_{i} \neq \pi_{j}$ for Policy 9 .

Table D.2 and Figure D.1 show the counterexample. Table D.2 shows a system $N=\{1,2\}$ together with the energy consumption and activity function $a(S)$ for all possible configurations of this system. Figure D.1 shows the placement of the activities in time for the period $T=\left[t_{1}, t_{5}\right]: s_{1}=$ $\left.\left[\left(t_{1}, 1\right),\left(t_{3}, 0\right)\right], s_{2}=\left[\left(t_{2}, 1\right),\left(t_{4}, 0\right)\right)\right]$, where $t_{1}=0, t_{2}=2, t_{3}=4, t_{4}=7$ and $t_{5}=10$.

\section{Event}

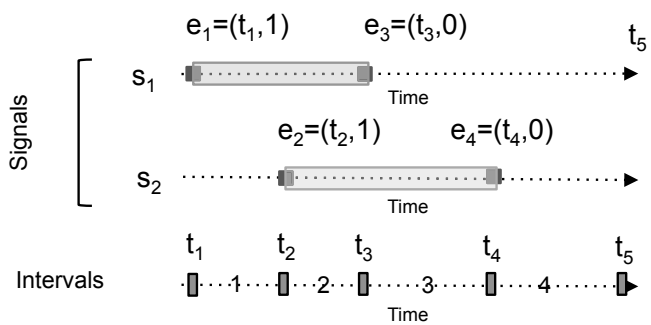

Figure D.1: Counterexample used in the proofs for symmetry for Policies 9 and 10 .

Since $E(\{1\})=E(\{2\})$ for $S=\emptyset$ the antecedent for the symmetry requirement obviously holds. From Equation 8.11 we have that:

$$
\begin{gathered}
\pi_{1}=\frac{1}{10}\left[2+\frac{1}{2}(4-2)+0+\frac{1}{2}(10-7)\right] E(N)=1.35 \\
\pi_{2}=\frac{1}{10}\left[0+\frac{1}{2}(4-2)+(7-4)+\frac{1}{2}(10-7)\right] E(N)=1.65
\end{gathered}
$$


Hence, $\pi_{1} \neq \pi_{2}$. Policy 9 does not satisfy symmetry.

Theorem 5. Policy 10 (Last active) does not always satisfy symmetry.

Proof. A counterexample is enough to prove the theorem. We show that $E(S \cup\{i\})=E(S \cup\{j\})$ for all $S \subseteq N \backslash\{i, j\}$ holds for the counterexample and prove that $\pi_{i} \neq \pi_{j}$ for Policy 10 .

Table D.2 shows a system $N=\{1,2\}$ together with the energy consumption and activity function $a(S)$ for all possible configurations of this system. Figure D.1 shows an illustration of the counterexample for the pe$\left.\operatorname{riod} T=\left[t_{1}, t_{5}\right]: s_{1}=\left[\left(t_{1}, 1\right),\left(t_{3}, 0\right)\right], s_{2}=\left[\left(t_{2}, 1\right),\left(t_{4}, 0\right)\right)\right]$, where $t_{1}=0$, $t_{2}=2, t_{3}=4, t_{4}=7$ and $t_{5}=10$. There are four intervals and all entities are inactive in the last interval, i.e., $\sum_{i \in N} a(i, 4)=0$. Entity 2 is the last active entity for interval $4: l(2,4)=1$. For simplicity we assume $E(N, j)=\frac{E(N)}{|J|}$ for the four intervals $j \in J(|J|=4)$.

Since $E(\{1\})=E(\{2\})$ for $S=\emptyset$ the antecedent for the symmetry property is satisfied. The apportionment for entities 1 and 2 is computed from Equation 8.12:

$$
\begin{gathered}
\pi_{1}=\frac{E(N)}{|J|}+\frac{E(N)}{2|J|}+0+0=\frac{3 E(N)}{2|J|}=1.125 \\
\pi_{2}=0+\frac{E(N)}{2|J|}+\frac{E(N)}{|J|}+\frac{E(N)}{|J|}=\frac{5 E(N)}{2|J|}=1.875
\end{gathered}
$$

Hence, $\pi_{1} \neq \pi_{2}$. Policy 10 does not satisfy symmetry.

\section{D.2 Dummy player}

In this section we provide the proofs for the dummy player property. Policies $2,3,8,9$ and 10 do not satisfy the dummy player property. The dummy player property presented in section 8.4.1 is the following: if $E(S \cup\{i\})-$ $E(S)=E(\{i\})$ for all $S \subseteq N \backslash\{i\}$, then $\pi_{i}=E(\{i\})$.

Theorem 6. Policy 2 (Proportional to isolation) does not always satisfy dummy player.

Proof. It is enough to provide a counterexample to prove the theorem. We show that $E(S \cup\{i\})-E(S)=E(\{i\})$ for all $S \subseteq N \backslash\{i\}$ holds in the counterexample and show that $\pi_{i} \neq E(\{i\})$ for Policy 2 .

Table D.3 shows a system $N=\{1,2,3\}$ together with the energy consumption for all possible configurations of this system.

Table D.3: A game where entity 2 is a dummy player.

\begin{tabular}{|c||c|c|c|c|c|c|c|}
\hline$S$ & $\{1\}$ & $\{2\}$ & $\{3\}$ & $\{1,2\}$ & $\{1,3\}$ & $\{2,3\}$ & $\{1,2,3\}$ \\
\hline$E(S)$ & 4 & 2 & 3 & 6 & 5 & 5 & 7 \\
\hline
\end{tabular}

Entity 2 satisfies the antecedent for the dummy player property: 


$$
\begin{gathered}
E(\{1,2,3\})-E(\{1,3\})=E(\{2\}) \\
E(\{1,2\})-E(\{1\})=E(\{2\}) \\
E(\{2,3\})-E(\{3\})=E(\{2\}) \\
E(\{2\})-E(\emptyset)=E(\{2\})
\end{gathered}
$$

From Equation 8.4 and the values in Table D.3, the apportionment for the three entities is computed: $\pi=(3.11,1.55,2.33)$. Clearly $\pi_{2} \neq E(\{2\})$ and thus Policy 2 does not satisfy the dummy player property.

The normalisation factor of Equation 8.4 is the main reason for not satisfying dummy player for Policy 2 .

Theorem 7. Policy 3 (Marginal contribution) does not always satisfy dummy player.

Proof. It is enough to provide a counterexample to prove the theorem. We show that $E(S \cup\{i\})-E(S)=E(\{i\})$ for all $S \subseteq N \backslash\{i\}$ holds in the counterexample and prove that $\pi_{i} \neq E(\{i\})$ for Policy 3 .

We employ the same counterexample as in the previous proof: Table D.3 shows a system $N=\{1,2,3\}$ together with the energy consumption for all possible configurations of this system. Entity 2 satisfies the antecedent for the dummy player property.

From Equation 8.5 and the values in Table D.3 the apportionment for the three entities is computed: $\pi=(2.8,1.4,2.8)$. Clearly $\pi_{2} \neq E(\{2\})$ and thus Policy 3 does not satisfy the dummy player property.

Similarly to Policy 2, the normalisation factor of Equation 8.5 is the main reason for not satisfying dummy player for Policy 3.

Theorem 8. Policy 8 (Proportional to activity) does not always satisfy dummy player.

Proof. It is enough to provide a counterexample to prove the theorem. We show that $E(S \cup\{i\})-E(S)=E(\{i\})$ for all $S \subseteq N \backslash\{i\}$ holds in the counterexample and show that $\pi_{i} \neq E(\{i\})$ for Policy 8 .

Table D.3 shows a system $N=\{1,2,3\}$ together with the energy consumption for all possible configurations of the system. The values for the activity function are shown in Table D.4 which are the same as the $E(S)$ values.

Table D.4: Activity function values for the counterexample.

\begin{tabular}{|c||c|c|c|c|c|c|c|}
\hline$S$ & $\{1\}$ & $\{2\}$ & $\{3\}$ & $\{1,2\}$ & $\{1,3\}$ & $\{2,3\}$ & $\{1,2,3\}$ \\
\hline$a(S)$ & 4 & 2 & 3 & 6 & 5 & 5 & 7 \\
\hline
\end{tabular}

Entity 2 satisfies the antecedent for the dummy player property from the values of Table D. 3 as shown in the Theorem 6 . 
From Equation 8.10 the apportionment for the entities is computed: $\pi=$ $(3.11,1.55,2.33)$. Obviously $\pi_{2} \neq E(\{2\})$. Hence, Policy 8 does not satisfy the dummy player property.

Similarly to the Policies 2 and 3, the normalisation factor is the main reason for not satisfying the dummy player.

Theorem 9. Policy 9 (Time slicing) does not always satisfy dummy player.

Proof. It is enough to provide a counterexample to prove the theorem. We show that $E(S \cup\{i\})-E(S)=E(\{i\})$ for all $S \subseteq N \backslash\{i\}$ holds in the counterexample and show that $\pi_{i} \neq E(\{i\})$ for Policy 9 .

Tables D. 3 and D.4 show a system $N=\{1,2,3\}$ and the energy consumption and activity function for every configuration of the system. An illustration of the counterexample is shown in Figure D.2 for a period $T=\left[t_{1}, t_{7}\right]$ : $s_{1}=\left[\left(t_{1}, 1\right),\left(t_{3}, 0\right)\right], s_{2}=\left[\left(t_{5}, 1\right),\left(t_{6}, 0\right)\right], s_{3}=\left[\left(t_{2}, 1\right),\left(t_{4}, 0\right)\right]$, where $t_{1}=0$, $t_{2}=2, t_{3}=4, t_{4}=5, t_{5}=6, t_{6}=8$ and $t_{7}=10$. There are 6 intervals $(|J|=6)$ where there are no active entities for intervals 4 and 6 , i.e., $\sum_{i \in N} a(i, 4)=0$ and $\sum_{i \in N} a(i, 6)=0$.

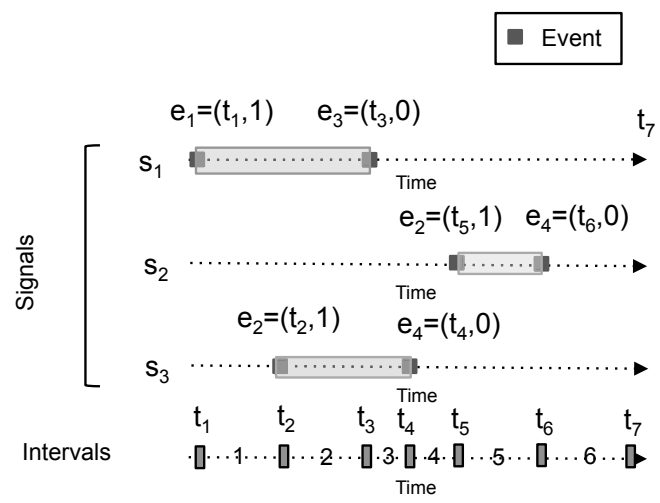

Figure D.2: Illustration of the counterexample used in the proofs for dummy player for Policies 9 and 10.

Entity 2 satisfies the antecedent for the dummy player property from the values of Table D.3 as shown in the Theorem 6 .

From Equation 8.11 the apportionment for the entities is computed:

$$
\begin{aligned}
& \pi_{1}=\frac{1}{10}\left[2+\frac{2}{2}+0+\frac{1}{3}+0+\frac{2}{3}\right] E(N)=2.8 \\
& \pi_{2}=\frac{1}{10}\left[0+0+0+\frac{1}{3}+2+\frac{2}{3}\right] E(N)=2.1 \\
& \pi_{3}=\frac{1}{10}\left[0+\frac{2}{2}+1+\frac{1}{3}+0+\frac{2}{3}\right] E(N)=2.1
\end{aligned}
$$

Clearly $\pi_{2} \neq E(\{2\})$, and thus Policy 9 does not satisfy the dummy player property.

Theorem 10. Policy 10 (Last active) does not always satisfy dummy player. 
Proof. It is enough to provide a counterexample to prove the theorem. We show that $E(S \cup\{i\})-E(S)=E(\{i\})$ for all $S \subseteq N \backslash\{i\}$ holds in the counterexample and show that $\pi_{i} \neq E(\{i\})$ for Policy 10.

Tables D. 3 and D. 4 show a system $N=\{1,2,3\}$ and the energy consumption and activity function for every configuration of the system. An illustration of the counterexample is shown in Figure D.2 for a period $T=\left[t_{1}, t_{7}\right]$ : $s_{1}=\left[\left(t_{1}, 1\right),\left(t_{3}, 0\right)\right], s_{2}=\left[\left(t_{5}, 1\right),\left(t_{6}, 0\right)\right], s_{3}=\left[\left(t_{2}, 1\right),\left(t_{4}, 0\right)\right]$, where $t_{1}=0$, $t_{2}=2, t_{3}=4, t_{4}=5, t_{5}=6, t_{6}=8$ and $t_{7}=10$. There are 6 intervals $(|J|=6)$ where there are no active entities for intervals 4 and 6 , i.e., $\sum_{i \in N} a(i, 4)=0$ and $\sum_{i \in N} a(i, 6)=0$. For these intervals we have that $l(3,4)=1$ and $l(2,6)=1$, i.e., entity 3 is the last active entity for interval 4 and entity 2 is the last active entity for interval 6 respectively. For simplicity we assume $E(N, j)=\frac{E(N)}{|J|}$ for the four intervals $j \in J(|J|=6)$.

Entity 2 satisfies the antecedent for the dummy player property from the values of Table D. 3 as shown in the Theorem 6 .

The apportionment for entities 1, 2 and 3 is computed from Equation 8.12:

$$
\begin{gathered}
\pi_{1}=\frac{E(N)}{|J|}+\frac{E(N)}{2|J|}+0+0+0+0=\frac{3 E(N)}{2|J|}=1.75 \\
\pi_{2}=0+0+0+0+\frac{E(N)}{|J|}+\frac{E(N)}{|J|}=\frac{2 E(N)}{|J|}=2.33 \\
\pi_{3}=0+\frac{E(N)}{2|J|}+\frac{E(N)}{|J|}+\frac{E(N)}{|J|}+0+0=\frac{5 E(N)}{2|J|}=2.92
\end{gathered}
$$

Obviously $\pi_{2} \neq E(\{2\})$ and thus Policy 10 does not satisfy the dummy player property.

Null player: Now we show that even though Policies 2, 3, 8 and 10 do not always satisfy dummy player, they satisfy a basic case of dummy player known as the null player property: if $E(S \cup\{i\})-E(S)=0$ for all $S \subseteq N \backslash\{i\}$, then $\pi_{i}=0$.

Theorem 11. Policy 2 (Proportional to isolation) always satisfies null player.

Proof. In the proof we assume $E(S \cup\{i\})-E(S)=0$ for all $S \subseteq N \backslash\{i\}$ and show $\pi_{i}=0$.

It follows from the antecedent of the null player property that $E(\{i\})=0$ for $S=\emptyset$. From Equation 8.4 and $E(\{i\})=0$ we have the following:

$$
\pi_{i}=\frac{E(\{i\})}{\sum_{j \in N} E(\{j\})} E(N)=\frac{0}{\sum_{j \in N} E(\{j\})} E(N)=0
$$

Thus, Policy 2 satisfies the null player property.

Theorem 12. Policy 3 (Marginal contribution) always satisfies null player. 
Proof. In the proof we assume $E(S \cup\{i\})-E(S)=0$ for all $S \subseteq N \backslash\{i\}$ and show $\pi_{i}=0$.

It follows from the antecedent of the null player property that $E(N)-$ $E(N \backslash\{i\})=0$ for $S=N \backslash\{i\}$. From Equation 8.5 and $E(N)-E(N \backslash\{i\})=0$ we have the following:

$$
\begin{aligned}
& \pi_{i}=\frac{E(N)-E(N \backslash\{i\})}{\sum_{j \in N}[E(N)-E(N \backslash\{j\})]} E(N) \\
= & \frac{0}{\sum_{j \in N}[E(N)-E(N \backslash\{j\})]} E(N)=0
\end{aligned}
$$

Hence, Policy 3 satisfies the null player property.

The notion of null player was defined in terms of energy. When we discuss surrogate-based policies we should envisage a surrogate type of null player property. Intuitively, it makes sense that if an entity does not have a surrogate attribute in a scenario (e.g., it does not have any activity), it does not consume energy in isolation and vice versa. Hence, $a(i)=0$ iff $E(\{i\})=0$.

Theorem 13. Policy 8 (Proportional to activity) always satisfies null player.

Proof. In the proof we assume $E(S \cup\{i\})-E(S)=0$ for all $S \subseteq N \backslash\{i\}$ and show $\pi_{i}=0$.

It follows from the antecedent of the null player property that $E(\{i\})=0$ for $S=\emptyset$. Since $E(\{i\})=0, a(i)=0$. From Equation 8.10 and $a(i)=0$ we have the following:

$$
\pi_{i}=\frac{a(i)}{\sum_{j \in N} a(j)} E(N)=\frac{0}{\sum_{j \in N} a(j)} E(N)=0
$$

Hence, Policy 8 satisfies the null player property.

Theorem 14. Policy 9 (Time slicing) does not always satisfy null player.

Proof. A counterexample is enough to prove the theorem. We show that $E(S \cup\{i\})-E(S)=0$ for all $S \subseteq N \backslash\{i\}$ holds in the counterexample and show $\pi_{i} \neq 0$.

Table D.5 and Figure D.3 show the counterexample. Table D.5 shows a system $N=\{1,2\}$ and the energy consumption and activity function for all possible configurations of this system. Figure D.3 shows the placement of the activities in time for the period $T=10: s_{1}=\left[\left(t_{1}, 1\right),\left(t_{2}, 0\right)\right]$ and $s_{2}=[]$ (no events), where $t_{1}=0, t_{2}=5$ and $t_{3}=10$. There are two intervals and in the last interval there is no active entity $\left(\sum_{i \in N} a(i, 2)=0\right)$.

Since $E(\{2\})-E(\{\emptyset\})=0, E(\{1,2\})-E(\{1\})=0$ and $a(2)=0$ from Table D.5 entity 2 satisfies the antecedent for the null player property.

From Equation 8.11 the apportionment is computed:

$$
\begin{aligned}
& \pi_{1}=\frac{1}{10}\left[5+\frac{5}{2}\right] E(N)=2.25 \\
& \pi_{2}=\frac{1}{10}\left[0+\frac{5}{2}\right] E(N)=0.75
\end{aligned}
$$


Table D.5: A counterexample used to show that Policy 9 does not satisfy then null player property.

\begin{tabular}{|c||c|c|c|}
\hline$S$ & $\{1\}$ & $\{2\}$ & $\{12\}$ \\
\hline$E(S)$ & 3 & 0 & 3 \\
\hline \hline$a(S)$ & 5 & 0 & 5 \\
\hline
\end{tabular}

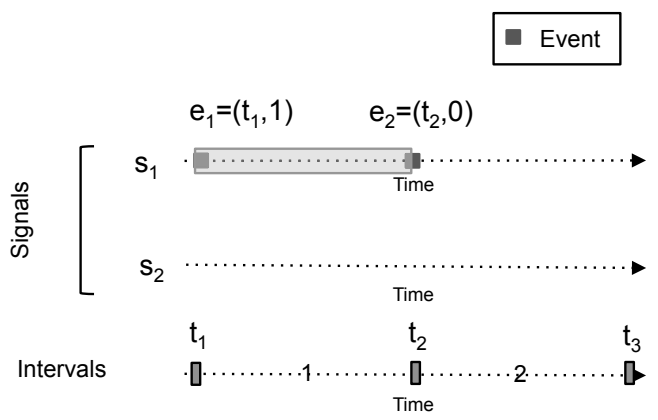

Figure D.3: Illustration of the counterexample used in the proof for null player for Policy 10.

Clearly $\pi_{2} \neq 0$ and thus Policy 9 does not satisfy the null player property.

Theorem 15. Policy 10 (Last active) always satisfies null player.

Proof. In the proof we assume $E(S \cup\{i\})-E(S)=0$ for all $S \subseteq N \backslash\{i\}$ and show $\pi_{i} \neq 0$.

From $E(S \cup\{i\})-E(S)=0$ for all $S \subseteq N \backslash\{i\}$ we have that $E(\{i\})=0$ for $S=\emptyset$. Since $E(\{i\})=0 a(i)=0$. Thus, $a(i, j)=0$ for all $j$. From Equation 8.12 there are two cases for $g(i, j)$ :

1. If $\sum_{i \in N} a(i, j)=0$, then $g(i, j)=l(i, j)$. Since $a(i, j)=0, l(i, j)=0$ for all $j$ (if an entity was not active, it cannot be the last active entity). Thus, $g(i, j)=0$.

2. If $\sum_{i \in N} a(i, j) \neq 0$, then $g(i, j)=\frac{a(i, j)}{\sum_{k \in N} a(k, j)}$. Since $a(i, j)=0$ we have that $g(i, j)=0$.

Since $g(i, j)=0$ for all $j, \pi_{i}=0$. Hence, Policy 10 satisfies the null player property.

Note that Policy 10 does not satisfy dummy player due to the last active entity mechanism, whereas it satisfies null player since an entity that never is active cannot be the last active entity. 


\section{D.3 Individual rationality}

In this section we provide the proofs for individual rationality. Policy 2 and 4 always satisfy individual rationality. Policies 3, 8, 9 and 10 do not always satisfy individual rationality. Individual rationality states that if $I(E) \neq \emptyset$ then $\pi_{i} \leq E(\{i\}), \forall i \in N$. It is well known that $\sum_{i \in N} E(\{i\}) \geq E(N)$ iff $I(E) \neq \emptyset$.

Theorem 16. Policy 2 (Proportional to isolation) always satisfies individual rationality.

Proof. In the proof we will assume $I(E) \neq \emptyset$ and show $\pi_{i} \leq E(\{i\}), \forall i \in N$.

It follows from the antecedent of individual rationality that $\sum_{i \in N} E(\{i\}) \geq$ $E(N)$. Thus:

$$
\frac{E(N)}{\sum_{i \in N} E(\{i\})} \leq 1
$$

Consequently, from Equation 8.4 we have that:

$$
\begin{gathered}
\pi_{i}=E(\{i\}) \frac{E(N)}{\sum_{j \in N} E(\{j\})} \\
\pi_{i} \leq E(\{i\})
\end{gathered}
$$

Hence, Policy 2 satisfies individual rationality.

Theorem 17. Policy 3 (Marginal contribution) does not always satisfy individual rationality.

Proof. A counterexample is enough to prove the theorem. We show that $I(E) \neq \emptyset$ holds in the counterexample and show $\pi_{i} \leq E(\{i\}), \forall i \in N$ is not satisfied for Policy 3.

Table D.6 shows a system $N=\{1,2,3\}$ and the energy consumption for all possible configurations of this system.

Table D.6: An example where Policy 3 does not satisfy individual rationality.

\begin{tabular}{|c||c|c|c|c|c|c|c|}
\hline$S$ & $\{1\}$ & $\{2\}$ & $\{3\}$ & $\{1,2\}$ & $\{1,3\}$ & $\{2,3\}$ & $\{1,2,3\}$ \\
\hline$E(S)$ & 2 & 1 & 2 & 2 & 2 & 2 & 4 \\
\hline
\end{tabular}

$\sum_{i \in N} E(\{i\}) \geq E(N)$ is satisfied from the values in Table D.6 $(2+1+2>$ $4)$, and thus $I(E) \neq \emptyset$ holds for the counterexample.

From Equation 8.5 and the values in Table D.6 the apportionment is computed: $\pi=(1.33,1.33,1.33)$. Clearly $\pi_{2}>E(\{2\})$, and thus Policy 3 does not satisfy individual rationality.

Since Policy 3 does not look at the energy consumption of the singletons, it fails to be individually rational. Instead, for the same game, Policy 2 results in $\pi=(1.6,0.8,1.6)$ which is individually rational. 
Theorem 18. Policy 4 (Isolation energy and remainder) always satisfies individual rationality.

Proof. In the proof we will assume $I(E) \neq \emptyset$ and show $\pi_{i} \leq E(\{i\}), \forall i \in N$.

It follows from the antecedent of individual rationality that $\sum_{i \in N} E(\{i\}) \geq$ $E(N)$. Thus:

$$
E(N)-\sum_{i \in N} E(\{i\}) \leq 0
$$

Consequently, from Equation 8.6 we have that:

$$
\begin{gathered}
\pi_{i}=E(\{i\})+\frac{E(N)-\sum_{j \in N} E(\{j\})}{|N|} \\
\pi_{i} \leq E(\{i\})
\end{gathered}
$$

Hence, Policy 4 satisfies individual rationality.

Theorem 19. Policy 8 (Proportional to activity) does not always satisfy individual rationality.

Proof. A counterexample is enough to prove the theorem. We show that $I(E) \neq \emptyset$ holds in the counterexample and prove that the following is not satisfied $\pi_{i} \leq E(\{i\}), \forall i \in N$ for Policy 8 .

Table D.6 shows a system $N=\{1,2,3\}$, the energy consumption and the activity function for all possible configurations of this system.

Table D.7: A counterexample used to show that Policies 8, 9 and 10 do not always satisfy individual rationality.

\begin{tabular}{|c||c|c|c|c|c|c|c|}
\hline$S$ & $\{1\}$ & $\{2\}$ & $\{3\}$ & $\{1,2\}$ & $\{1,3\}$ & $\{2,3\}$ & $\{1,2,3\}$ \\
\hline$E(S)$ & 2 & 1 & 2 & 2 & 2 & 2 & 4 \\
\hline \hline$a(S)$ & 4 & 3 & 4 & 7 & 6 & 6 & 8 \\
\hline
\end{tabular}

$\sum_{i \in N} E(\{i\}) \geq E(N)$ is satisfied from the values in Table D.7 $(2+1+2>$ $4)$, and thus $I(E) \neq \emptyset$ holds for the counterexample.

From Equation 8.10 and the values in Table D.7 the apportionment for the three entities is computed: $\pi=(1.45,1.09,1.45)$. Obviously $\pi_{2}>$ $E(\{2\})$. Policy 8 does not satisfy individual rationality.

Theorem 20. Policy 9 (Time slicing) does not always satisfy individual rationality.

Proof. A counterexample is enough to prove the theorem. We show that $I(E) \neq \emptyset$ holds in the counter example and prove that $\pi_{i} \leq E(\{i\}), \forall i \in N$ is not satisfied for Policy 9 .

Table D. 6 and Figure D.4 show the counterexample. Table D.6 shows a system $N=\{1,2,3\}$, the energy consumption and the activity function for 
all possible configurations of this system. Figure D.4 shows the placement of the activities in time for the period $T=10: s_{1}=\left[\left(t_{1}, 1\right),\left(t_{3}, 0\right)\right], s_{2}=$ $\left[\left(t_{4}, 1\right),\left(t_{6}, 0\right)\right]$ and $s_{3}=\left[\left(t_{2}, 1\right),\left(t_{5}, 0\right)\right]$, where $t_{1}=0, t_{2}=2, t_{3}=4, t_{4}=5$, $t_{5}=6, t_{6}=8$ and $t_{7}=10$. There are 6 intervals and for the last interval there is no active entity $\left(\sum_{i \in N} a(i, 6)=0\right)$.

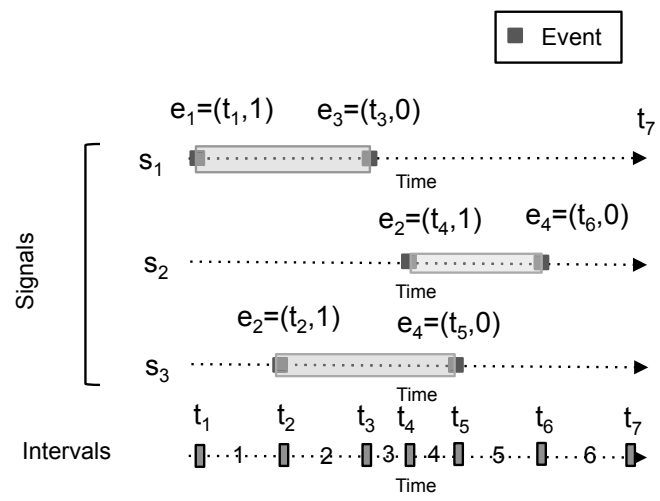

Figure D.4: Illustration of the counterexample used in the proofs for individual rationality for Policies 8, 9 and 10.

$\sum_{i \in N} E(\{i\}) \geq E(N)$ is satisfied from the values in Table D.7 $(2+1+2>$ $4)$, and thus $I(E) \neq \emptyset$ holds for the counterexample.

From Equation 8.11 the apportionment for the entities 1, 2 and 3 is computed:

$$
\begin{aligned}
& \pi_{1}=\frac{1}{10}\left[2+\frac{2}{2}+0+0+0+\frac{2}{3}\right] E(N)=1.46 \\
& \pi_{2}=\frac{1}{10}\left[0+0+0+\frac{1}{2}+2+\frac{2}{3}\right] E(N)=1.26 \\
& \pi_{3}=\frac{1}{10}\left[0+\frac{2}{2}+1+\frac{1}{2}+0+\frac{2}{3}\right] E(N)=1.26
\end{aligned}
$$

Clearly $\pi_{2}>E(\{2\})$, and thus Policy 9 does not satisfy individual rationality.

Theorem 21. Policy 10 (Last active) does not always satisfy individual rationality .

Proof. A counterexample is enough to prove the theorem. We show that $I(E) \neq \emptyset$ holds in the counter example and prove that $\pi_{i} \leq E(\{i\}), \forall i \in N$ is not satisfied for Policy 10.

Table D.6 and Figure D.4 show the counterexample. Table D. 6 shows a system $N=\{1,2,3\}$, the energy consumption and the activity function for all possible configurations of this system. Figure D.4 shows the placement of the activities in time for the period $T=10: s_{1}=\left[\left(t_{1}, 1\right),\left(t_{3}, 0\right)\right], s_{2}=$ $\left[\left(t_{4}, 1\right),\left(t_{6}, 0\right)\right]$ and $s_{3}=\left[\left(t_{2}, 1\right),\left(t_{5}, 0\right)\right]$, where $t_{1}=0, t_{2}=2, t_{3}=4, t_{4}=5$, $t_{5}=6, t_{6}=8$ and $t_{7}=10$. There are 6 intervals and for the last interval there is no active entity $\left(\sum_{i \in N} a(i, 6)=0\right)$. Thus, $l(2,6)=1$. For simplicity we assume $E(N, j)=\frac{E(N)}{|J|}$ for the six intervals $j \in J(|J|=6)$. 
$\sum_{i \in N} E(\{i\}) \geq E(N)$ is satisfied from the values in Table D.7 $(2+1+2>$ $4)$, and thus $I(E) \neq \emptyset$ holds for the counterexample.

From Equation 8.12 the apportionment for the counterexample is computed:

$$
\begin{gathered}
\pi_{1}=\frac{E(N)}{|J|}+\frac{E(N)}{2|J|}+0+0+0+0=\frac{3 E(N)}{2|J|}=1 \\
\pi_{2}=0+0+0+\frac{E(N)}{2|J|}+\frac{E(N)}{|J|}+\frac{E(N)}{|J|}=\frac{5 E(N)}{2|J|}=1.66 \\
\pi_{3}=0+\frac{E(N)}{2|J|}+\frac{E(N)}{|J|}+\frac{E(N)}{2|J|}+0+0=\frac{2 E(N)}{|J|}=1.33
\end{gathered}
$$

Obviously $\pi_{2}>E(\{2\})$ and thus Policy 10 does not satisfy individual rationality.

\section{D.4 Coalitional rationality}

In this section we provide the proofs for the conditional rationality property which states that if $C(E) \neq \emptyset$ then $\sum_{i \in S} \pi_{i} \leq E(S) \forall S \subseteq N$. The property includes individual rationality when $|S|=1$, and thus Policies $1,3,8,9$ and 10 that do not satisfy individual rationality do not satisfy coalitional rationality either. In this section we provide the proof showing that Policy 4 does not satisfy coalitional rationality even though it satisfies individual rationality.

Theorem 22. Policy 4 (Isolation energy and remainder) does not always satisfy coalitional rationality.

Proof. A counterexample is enough to prove the theorem. We show that $C(E) \neq \emptyset$ in the counterexample and show that $\sum_{i \in S} \pi_{i} \leq E(S) \forall S \subseteq N$ is not satisfied for Policy 4.

Table D. 8 shows a system $N=\{1,2,3\}$ and the energy consumption for every possible system configuration.

Table D.8: Counterexample to prove that Policy 4 does not satisfy coalitional rationality.

\begin{tabular}{|c||c|c|c|c|c|c|c|}
\hline$S$ & $\{1\}$ & $\{2\}$ & $\{3\}$ & $\{1,2\}$ & $\{1,3\}$ & $\{2,3\}$ & $\{1,2,3\}$ \\
\hline$E(S)$ & 3 & 3 & 4 & 4.5 & 5.5 & 6 & 8 \\
\hline
\end{tabular}

An individually rational apportionment satisfies the following inequalities:

$$
\begin{aligned}
& \pi_{1} \leq E(\{1\}) \\
& \pi_{2} \leq E(\{2\}) \\
& \pi_{3} \leq E(\{3\})
\end{aligned}
$$


Additionally, a coalitionally rational apportionment satisfies also the following inequalities:

$$
\begin{gathered}
\pi_{1}+\pi_{2} \leq E(\{1,2\}) \\
\pi_{1}+\pi_{3} \leq E(\{1,3\}) \\
\pi_{2}+\pi_{3} \leq E(\{2,3\}) \\
\pi_{1}+\pi_{2}+\pi_{3} \leq E(\{1,2,3\})
\end{gathered}
$$

The counterexample in Table D.8 satisfies $C(E) \neq \emptyset$ since the inequality system has at least an answer (e.g., $\pi=(2,2.5,3.5))$.

From Equation 8.6 the apportionment for entities 1, 2 and 3 is computed: $\pi=(2.33,2.33,3.33) . \quad \pi$ is individually rational since $\pi_{1}<E(\{1\}), \pi_{2}<$ $E(\{2\})$ and $\pi_{3}<E(\{3\})$. For coalitional rationality we have the following:

$$
\begin{gathered}
2.33+2.33>E(\{1,2\}) \\
2.33+3.33>E(\{1,3\}) \\
2.33+3.33<E(\{2,3\}) \\
2.33+2.33+3.33<E(\{1,2,3\})
\end{gathered}
$$

Clearly $\pi_{1}+\pi_{2}>E(\{1,2\})$ and $\pi_{2}+\pi_{3}>E(\{1,3\})$, and thus Policy 4 does not satisfy coalitional rationality.

The main reason for Policy 4 to not satisfy coalitional rationality is that Equation 8.6 only consider the value of the singleton coalitions to apportion the energy consumption of the grand coalition.

\section{D.5 Entity desirability}

In this section we provide the proofs showing that Policies 1, 2, 3 and 4 satisfy entity desirability and Policies 8, 9 and 10 do not satisfy entity desirability.

Entity desirability states that $i \succeq j \Longrightarrow \pi_{i} \leq \pi_{j}$, where $i \succeq j$ is defined as $E(S \cup\{i\}) \leq E(S \cup\{j\}) \forall S \subseteq N \backslash\{i, j\}$.

Theorem 23. Policy 1 always satisfies entity desirability.

Proof. In order to prove the theorem we assume $i \succeq j$ and show $\pi_{i} \leq \pi_{j}$ for Equation 8.3.

From Equation 8.3 we have that $\pi_{i}=\pi_{j}$ for all $i, j \in N$. Hence, Policy 1 trivially satisfies entity desirability.

Theorem 24. Policy 2 (Proportional to isolation) always satisfies entity desirability. 
Proof. In order to prove it we assume $i \succeq j$ and show $\pi_{i} \leq \pi_{j}$ for Equation 8.4 .

It follows from the antecedent of entity desirability that $E(\{i\}) \leq E(\{j\})$ for $S=\emptyset$. From Equation 8.4 and $E(\{i\}) \leq E(\{j\})$ we have the following for $i$ and $j$ :

$$
\begin{aligned}
E(\{i\}) \frac{E(N)}{\sum_{k \in N} E(\{k\})} & \leq E(\{j\}) \frac{E(N)}{\sum_{k \in N} E(\{k\})} \\
\pi_{i} & \leq \pi_{j}
\end{aligned}
$$

Hence, Policy 2 satisfies entity desirability.

Theorem 25. Policy 3 (Marginal contribution) always satisfies entity desirability.

Proof. In order to prove the theorem we assume $i \succeq j$ and show $\pi_{i} \leq \pi_{j}$ for Equation 8.5.

We know that $E(S \cup\{i\})=E(N \backslash\{j\})$ and $E(S \cup\{j\})=E(N \backslash\{i\})$ for $S=N \backslash\{i, j\}$. Thus, it follows from the assumption that $E(N \backslash\{j\}) \leq$ $E(N \backslash\{i\})$. Hence, $E(N)-E(N \backslash\{j\}) \geq E(N)-E(N \backslash\{i\})$.

From Equation 8.5 and $E(N)-E(N \backslash\{i\}) \leq E(N)-E(N \backslash\{j\})$ we have the following for $i$ and $j$ :

$$
\begin{aligned}
\frac{E(N)-E(N \backslash\{i\})}{\sum_{k \in N}[E(N)-E(N \backslash\{k\})]} E(N) & \leq \frac{E(N)-E(N \backslash\{j\})}{\sum_{k \in N}[E(N)-E(N \backslash\{k\})]} E(N) \\
\pi_{i} & \leq \pi_{j}
\end{aligned}
$$

Thus, Policy 3 satisfies entity desirability.

Theorem 26. Policy 4 always satisfies entity desirability.

Proof. In order to prove the theorem we assume $i \succeq j$ and prove that for Equation 8.6 we have $\pi_{i} \leq \pi_{j}$.

From $i \succeq j$ it follows that $E(\{i\}) \leq E(\{j\})$ for $S=\emptyset$. From Equation 8.6 and $E(\{i\}) \leq E(\{j\})$ we have that:

$$
\begin{aligned}
E(\{i\})+\frac{E(N)-\sum_{k \in N} E(\{k\})}{|N|} & \leq E(\{j\})+\frac{E(N)-\sum_{k \in N} E(\{k\})}{|N|} \\
\pi_{i} & \leq \pi_{j}
\end{aligned}
$$

Hence, Policy 4 satisfies entity desirability.

Theorem 27. Policy 8 (Proportional to activity) does not always satisfy entity desirability.

Proof. It is enough to provide a counterexample to prove the theorem. We show $i \succeq j$ in the counterexample and prove $\pi_{i}>\pi_{j}$.

Table D.9 shows a system $N=\{1,2\}$, the energy consumption and the activity function for every possible configuration of this system.

We know that $S=N \backslash\{1,2\}=\emptyset$. Thus, $1 \succeq 2$ is $E(S \cup\{1\}) \leq E(S \cup\{2\})$ for $S=\emptyset$, i.e., $E(\{1\}) \leq E(\{2\})$. This inequality holds for the values in Table D.9. Thus, $1 \succeq 2$ holds in the counterexample. 
Table D.9: A counterexample used to show that Policies 8, 9 and 10 do not satisfy entity desirability.

\begin{tabular}{|c||c|c|c|}
\hline$S$ & $\{1\}$ & $\{2\}$ & $\{12\}$ \\
\hline$E(S)$ & 4 & 5 & 3 \\
\hline \hline$a(S)$ & 4 & 3 & 6 \\
\hline
\end{tabular}

From Equation 8.10 and the values in Table D.9 the apportionment for the entities is computed: $\pi=(1.72,1.28)$. Clearly $\pi_{1}>\pi_{2}$, and thus Policy 8 does not satisfy entity desirability.

Theorem 28. Policy 9 (Time slicing) does not always satisfy entity desirability.

Proof. It is enough to provide a counterexample to prove the theorem. We show $i \succeq j$ in the counterexample and prove $\pi_{i}>\pi_{j}$ for Policy 9 .

Table D.9 and Figure D.5 show the counterexample. Table D.9 shows a system $N=\{1,2\}$, the energy consumption and the activity function for every possible configuration of this system. Figure D.5 shows the placement of the activities in time for the period $T=10: s_{1}=\left[\left(t_{2}, 1\right),\left(t_{4}, 0\right)\right]$ and $s_{2}=\left[\left(t_{1}, 1\right),\left(t_{3}, 0\right)\right]$ where $t_{1}=0, t_{2}=2, t_{3}=3, t_{4}=6$ and $t_{5}=10$. There are four intervals and there is no active entity in the last interval $\left(\sum_{i \in N} a(i, 4)=0\right)$.

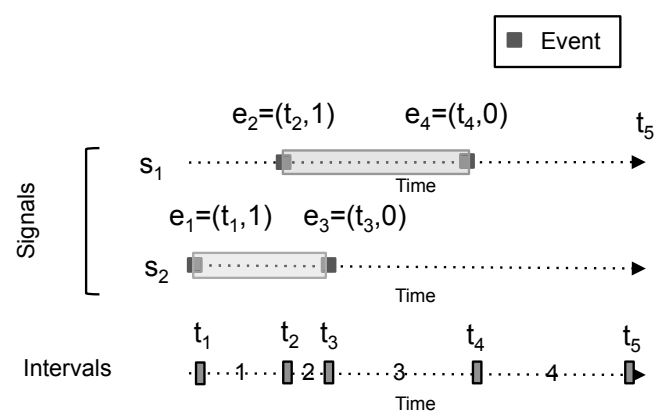

Figure D.5: Counterexample used to show that Policies 8, 9 and 10 do not satisfy entity desirability.

We know that $S=N \backslash\{1,2\}=\emptyset$. Thus, $1 \succeq 2$ is $E(S \cup\{1\}) \leq E(S \cup\{2\})$ for $S=\emptyset$, i.e., $E(\{1\}) \leq E(\{2\})$. This inequality holds for the values in Table D.9. Thus, $1 \succeq 2$ holds in the counterexample.

From Equation 8.11 the apportionment for entities 1 and 2 is computed:

$$
\begin{aligned}
& \pi_{1}=\frac{1}{10}\left[0+\frac{1}{2}+3+\frac{4}{2}\right] E(N)=1.65 \\
& \pi_{2}=\frac{1}{10}\left[2+\frac{1}{2}+0+\frac{4}{2}\right] E(N)=1.35
\end{aligned}
$$


Obviously $\pi_{1}>\pi_{2}$, and thus Policy 9 does not satisfy entity desirability.

Theorem 29. Policy 10 (Last active) does not always satisfy entity desirability.

Proof. It is enough to provide a counterexample to prove the theorem. We show $i \succeq j$ in the counterexample and prove $\pi_{i}>\pi_{j}$ for Policy 10 .

Table D.9 and Figure D.5 show the counterexample. Table D.9 shows a system $N=\{1,2\}$, the energy consumption and the activity function for every possible configuration of this system. Figure D.5 shows the placement of the activities in time for the period $T=10: s_{1}=\left[\left(t_{2}, 1\right),\left(t_{4}, 0\right)\right]$ and $s_{2}=\left[\left(t_{1}, 1\right),\left(t_{3}, 0\right)\right]$ where $t_{1}=0, t_{2}=2, t_{3}=3, t_{4}=6$ and $t_{5}=10$. There are four intervals and there is no active entity in the last interval $\left(\sum_{i \in N} a(i, 4)=0\right) . l(1,4)=1$ since entity 1 is the last active entity for interval 4. For simplicity we assume $E(N, j)=\frac{E(N)}{|J|}$ for the four intervals $j \in J(|J|=4)$.

We know that $S=N \backslash\{1,2\}=\emptyset$. Thus, $1 \succeq 2$ is $E(S \cup\{1\}) \leq E(S \cup\{2\})$ for $S=\emptyset$, i.e., $E(\{1\}) \leq E(\{2\})$. This inequality holds for the values in Table D.9. Thus, $1 \succeq 2$ holds in the counterexample.

From Equation 8.12 the apportionment for the counterexample is computed:

$$
\begin{gathered}
\pi_{1}=0+\frac{E(N)}{2|J|}+\frac{E(N)}{|J|}+\frac{E(N)}{|J|}=\frac{5 E(N)}{2|J|}=1.875 \\
\pi_{2}=\frac{E(N)}{|J|}+\frac{E(N)}{2|J|}+0+0=\frac{3 E(N)}{2|J|}=1.125
\end{gathered}
$$

Clearly $\pi_{1}>\pi_{2}$, and thus Policy 10 does not satisfy entity desirability.

\section{D.6 Activity-based fairness}

For systems where the energy information might not be available, it is relevant to analyse whether the surrogate-based policies satisfy fairness properties based on the available information: activity. In this section we analyse the surrogate-based policies considering a variation of the fairness criteria where the antecedents of the properties are stated in terms of activity.

We define the activity-based fairness criteria translating the antecedent from energy to activity when possible:

- Activity-based symmetry: if $a(S \cup\{i\})=a(S \cup\{j\})$ for all $S \subseteq$ $N \backslash\{i, j\}$, then $\pi_{i}=\pi_{j}$.

- Activity-based dummy player: if $a(S \cup\{i\})-a(S)=a(\{i\})$ for all $S \subseteq N \backslash\{i\}$, then $\pi_{i}=E(\{i\})$. Note that the apportionment policies divide the energy consumption, and thus the consequent of the property remains $\pi_{i}=E(\{i\})$ and not $\pi_{i}=a(\{i\})$, since the latter would imply that the apportionment policy apportions activity which is incorrect. 
- Activity-based individual rationality: if $I(E) \neq \emptyset$ and $\sum_{i \in N} a(i) \geq$ $a(N)$ then $\pi_{i} \leq E(\{i\}), \forall i \in N . I(E) \neq \emptyset$ is a required precondition for an apportionment that satisfies $\pi_{i} \leq E(\{i\}), \forall i \in N$ and it is well known that $I(E) \neq \emptyset$ iff $\sum_{i \in N} E(\{i\}) \geq E(N)$. Thus, an individually rational apportionment has still requirements in terms of energy given the consequent.

- Activity-based entity desirability: $i \succeq_{a} j \Longrightarrow \pi_{i} \leq \pi_{j}$, where $i \succeq_{a} j$ is defined as $a(S \cup\{i\}) \leq a(S \cup\{j\}) \forall S \subseteq N \backslash\{i, j\}$.

\section{D.6.1 Activity-based symmetry}

We provide the proofs that show that Policy 8 and 9 satisfy activity-based symmetry, whereas Policy 10 does not satisfy the property.

Theorem 30. Policy 8 (Proportional to activity) always satisfies activitybased symmetry.

Proof. In the proof we will assume $a(S \cup\{i\})=a(S \cup\{j\})$ for all $S \subseteq$ $N \backslash\{i, j\}$, and show $\pi_{i}=\pi_{j}$ for Policy 8 .

From Equation 8.10 the apportionment for entities $i$ and $j$ is the following:

$$
\begin{aligned}
& \pi_{i}=a(\{i\}) \frac{a(N)}{\sum_{k \in N} a(\{k\})} \\
& \pi_{j}=a(\{j\}) \frac{a(N)}{\sum_{k \in N} a(\{k\})}
\end{aligned}
$$

It follows from the antecedent of activity-based symmetry that $a(i)=a(j)$ for $S=\emptyset$. From Equation 8.10 and $a(i)=a(j)$ we have that:

$$
\pi_{i}=a(i) \frac{a(N)}{\sum_{k \in N} a(k)}=a(j) \frac{a(N)}{\sum_{k \in N} a(k)}=\pi_{j}
$$

Policy 8 satisfies symmetry.

Theorem 31. Policy 9 (Time slicing) always satisfies activity-based symmetry.

Proof. In the proof we will assume $a(S \cup\{i\})=a(S \cup\{j\})$ for all $S \subseteq$ $N \backslash\{i, j\}$, and show $\pi_{i}=\pi_{j}$ for Policy 9 .

From Equation 8.11 the apportionment for entities $i$ and $k$ is computed using the function $f(i, j)$ which has two cases. We analyse each case separately.

1. If $\sum_{i \in N} a(i, j)=0$, then $f(i, j)=f(k, j)=\frac{1}{|N|}$ for entities $i$ and $k$.

2. If $\sum_{i \in N} a(i, j) \neq 0$, then $f(i, j)$ and $f(k, j)$ are the following:

$$
\begin{aligned}
& f(i, j)=\frac{a(i, j)}{\sum_{m \in N} a(m, j)} \\
& f(k, j)=\frac{a(k, j)}{\sum_{m \in N} a(m, j)}
\end{aligned}
$$


It follows from the antecedent $a(S \cup\{i\})=a(S \cup\{j\})$ for all $S \subseteq$ $N \backslash\{i, j\}$ that $a(i, j)=a(k, j)$. From Equation 8.10 and $a(i, j)=a(k, j)$ we have that:

$$
f(i, j)=\frac{a(i, j)}{\sum_{m \in N} a(m, j)}=\frac{a(k, j)}{\sum_{m \in N} a(m, j)}=f(k, j)
$$

Since $f(i, j)=f(k, j)$ we have that $\pi_{i}=\pi_{k}$, and thus Policy 9 satisfies symmetry.

Theorem 32. Policy 10 (Last active) does not always satisfy activity-based symmetry.

Proof. A counterexample is enough to prove the theorem. We show that $a(S \cup\{i\})=a(S \cup\{j\})$ for all $S \subseteq N \backslash\{i, j\}$ holds in the conterexample and show $\pi_{i} \neq \pi_{j}$ for Policy 10 .

Figure D. 6 shows the counterexample for the system $N=\{1,2\}$ and the placement of the activity of each entity in time for the period $T=\left[t_{1}, t_{5}\right]$ : $\left.s_{1}=\left[\left(t_{1}, 1\right),\left(t_{3}, 0\right)\right], s_{2}=\left[\left(t_{2}, 1\right),\left(t_{4}, 0\right)\right)\right]$, where $t_{1}=0, t_{2}=3, t_{3}=4$, $t_{4}=7$ and $t_{5}=10$. There are four intervals where all entities are inactive in the last interval, i.e., $\sum_{i \in N} a(i, 4)=0 . l(2,4)=1$ since entity is the last active entity for interval 4 . For simplicity $E(N, j)=\frac{E(N)}{|J|}$ for the four intervals $j \in J(|J|=4)$ and $E(N)>0$.

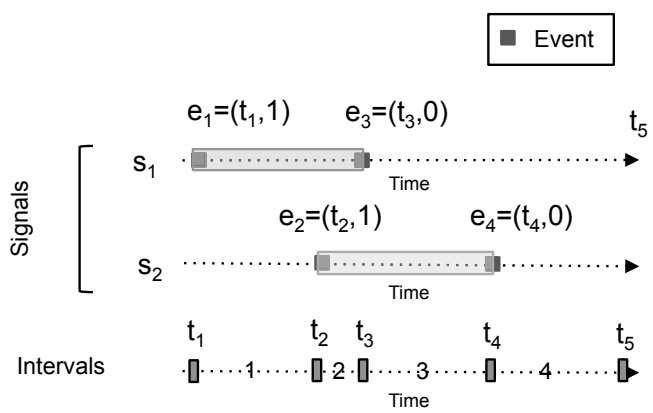

Figure D.6: Counterexample used in the proofs for activity-based symmetry for Policy 10.

The antecedent for the activity-based symmetry property holds since $a(1)=a(2)=4$. The apportionment for entities 1 and 2 is computed from Equation 8.12:

$$
\begin{gathered}
\pi_{1}=\frac{E(N)}{|J|}+\frac{E(N)}{2|J|}+0+0=\frac{3 E(N)}{2|J|} \\
\pi_{2}=0+\frac{E(N)}{2|J|}+\frac{E(N)}{|J|}+\frac{E(N)}{|J|}=\frac{5 E(N)}{2|J|}
\end{gathered}
$$

Clearly $\pi_{1} \neq \pi_{2}$, and thus Policy 10 does not satisfy activity-based symmetry. 


\section{D.6.2 Activity-based dummy player}

Theorems 8, 9 and 10 show that Policies 8, 9 and 10 do not satisfy the activity-based dummy player since the counterexamples show $a(S \cup\{i\})-$ $a(S)=a(\{i\})$ for all $S \subseteq N \backslash\{i\}$ and prove $\pi_{i} \neq E(\{i\})$ for $i=2$.

\section{D.6.3 Activity-based individual rationality}

Theorems 19, 20 and 21 show that Policies 8, 9 and 10 do not satisfy the activity-based individual rationality since in the counterexamples we assume $I(E) \neq \emptyset$ and $\sum_{i \in N} a(i) \geq a(N)$, and show that $\pi_{i} \leq E(\{i\})$ is not satisfied for all $i \in N$.

\section{D.6.4 Activity-based entity desirability}

We provide the proofs showing that Policies 8 and 9 satisfy activity-based entity desirability and Policy 10 does not satisfy the property.

Theorem 33. Policy 8 (Proportional to activity) always satisfies activitybased entity desirability.

Proof. In order to prove the theorem we assume $i \succeq j$ and prove $\pi_{i} \leq \pi_{j}$ for Policy 8.

It follows from the antecedent of activity-based entity desirability that $a(i) \leq a(j)$ for $S=\emptyset$. From Equation 8.4 and $a(i) \leq a(j)$ we have the following for $i$ and $j$ :

$$
\begin{aligned}
a(i) \frac{E(N)}{\sum_{k \in N} a(k)} & \leq a(j) \frac{E(N)}{\sum_{k \in N} a(k)} \\
\pi_{i} & \leq \pi_{j}
\end{aligned}
$$

Hence, Policy 8 satisfies entity desirability.

Theorem 34. Policy 9 (Time slicing) satisfies activity-based entity desirability.

Proof. In the proof we will assume $a(S \cup\{i\}) \leq a(S \cup\{j\})$ for all $S \subseteq$ $N \backslash\{i, j\}$, and prove $\pi_{i} \leq \pi_{j}$ for Policy 9 .

From Equation 8.11 the apportionment for entities $i$ and $k$ is computed using the function $f(i, j)$ which has two cases. We analyse each case separately.

1. If $\sum_{i \in N} a(i, j)=0$, then $f(i, j)=f(k, j)=\frac{1}{|N|}$ for entities $i$ and $k$.

2. If $\sum_{i \in N} a(i, j) \neq 0$, then $f(i, j)$ and $f(k, j)$ are the following:

$$
\begin{aligned}
& f(i, j)=\frac{a(i, j)}{\sum_{m \in N} a(m, j)} \\
& f(k, j)=\frac{a(k, j)}{\sum_{m \in N} a(m, j)}
\end{aligned}
$$


It follows from the antecedent $a(S \cup\{i\}) \leq a(S \cup\{j\})$ for all $S \subseteq N \backslash$ $\{i, j\}$ that $a(i, j) \leq a(k, j)$. From Equation 8.10 and $a(i, j) \leq a(k, j)$ we have that:

$$
\begin{aligned}
\frac{a(i, j)}{\sum_{m \in N} a(m, j)} & \leq \frac{a(k, j)}{\sum_{m \in N} a(m, j)} \\
f(i, j) & \leq f(k, j)
\end{aligned}
$$

Since $f(i, j) \leq f(k, j)$ for all $j$ intervals we have that $\pi_{i} \leq \pi_{k}$. Thus, Policy 9 satisfies symmetry.

Theorem 35. Policy 10 (Last active) does not always satisfy activity-based entity desirability.

Proof. A counterexample is enough to prove the theorem. We show that $i \succeq j$ holds in the counterexample and prove $\pi_{i}>\pi_{j}$ for Policy 10 .

Figure D.7 shows the counterexample for a system $N=\{1,2\}$ and the placement of the activity of the entities in time in the period $\left[t_{1}, t_{5}\right]: s_{1}=$ $\left[\left(t_{1}, 1\right),\left(t_{3}, 0\right)\right], s_{2}=\left[\left(t_{2}, 1\right),\left(t_{4}, 0\right)\right]$, where $t_{1}<t_{2}<t_{3}<t_{4}<t_{5}$ and $\left(t_{3}-t_{1}\right)>\left(t_{4}-t_{2}\right)$. There are four intervals and all the entities are inactive in the fourth interval, i.e., $\sum_{i \in N} a(i, 4)=0$. Thus, $l(1,4)=0$ and $l(2,4)=1$. For simplicity $E(N, j)=\frac{E(N)}{|J|}$ for the four intervals $j \in J(|J|=4)$.

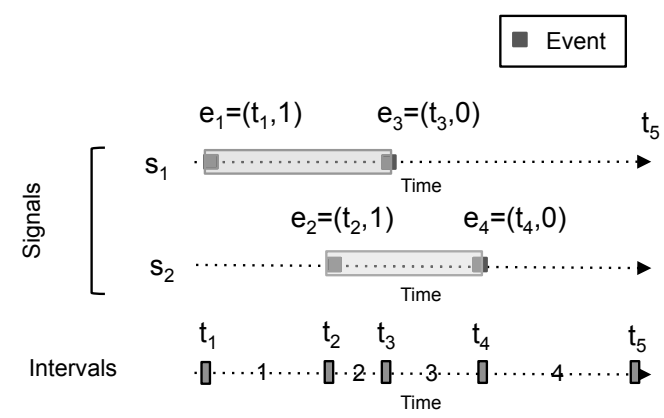

Figure D.7: Counterexample showing that Policy 10 does not satisfy entity desirability.

Since $\left(t_{3}-t_{1}\right)>\left(t_{4}-t_{2}\right)$ we have that $a(2)<a(1)$. Thus $2 \succeq 1$ holds in the counterexample.

From Equation 8.12 the apportionment for the entities is computed:

$$
\begin{gathered}
\pi_{1}=\frac{E(N)}{|J|}+\frac{E(N)}{2|J|}+0+0=\frac{3}{8} E(N) \\
\pi_{2}=0+\frac{E(N)}{2|J|}+\frac{E(N)}{|J|}+\frac{E(N)}{|J|}=\frac{5}{8} E(N)
\end{gathered}
$$

Clearly $\pi_{1}<\pi_{2}$ even if $2 \succeq 1$, and thus Policy 10 does not satisfy activitybased entity desirability. 


\section{Bibliography}

[1] 3GPP TS 25.331. Radio Resource Control (RRC), Protocol Specification, accessed 20th February, 2014.

http://www . 3gpp.org/DynaReport/25331.htm.

[2] 3GPP TS 36.331. Evolved Universal Terrestrial Radio Access (EUTRA), Radio Resource Control (RRC), Protocol Specification, accessed 20th February, 2014.

http://www.3gpp.org/DynaReport/36331.htm.

[3] Y. Agarwal, R. Chandra, A. Wolman, P. Bahl, K. Chin, and R. Gupta. Wireless wakeups revisited: energy management for VoIP over Wi-Fi smartphones. In Proceedings of the 5th International Conference on Mobile Systems, Applications and Services, MobiSys '07, pages 179191. ACM, 2007.

[4] B. Aggarwal, P. Chitnis, A. Dey, K. Jain, V. Navda, V. N. Padmanabhan, R. Ramjee, A. Schulman, and N. Spring. Stratus: Energyefficient mobile communication using cloud support. In Proceedings of the ACM SIGCOMM 2010 Conference, volume 41, pages 477-478. ACM, 2010.

[5] Agilent Technologies. Agilent 66321D Power Supply, accessed 20th February, 2014.

http://www.home.agilent.com/upload/cmc_upload/All/

66300series_datasheet_Jan07.pdf?\&cc $=$ SE\&lc=eng.

[6] Agilent Technologies. Agilent Digital Multimeters, accessed 20th February, 2014.

http://www .home.agilent.com/en/pc-1000000189\%3Aepsg\%

$3 \mathrm{Apgr} /$ digital-multimeter $-\mathrm{dmm} ? \& \mathrm{cc}=\mathrm{SE} \& \mathrm{l} \mathrm{c}=\mathrm{eng}$.

[7] L. Al-Kanj and Z. Dawy. Impact of network parameters on the design of energy-aware cooperative content distribution protocols. Transactions on Emerging Telecommunications Technologies, 24(3):317-330, John Wiley \& Sons, Ltd., 2013.

[8] A. Alesand. Emulating 3G Network Characteristics on WiFi Networks. Master Thesis. Linköping University, 2015. 
[9] M. Almquist and V. Almquist. A study on Android games: 3G energy consumption, CPU-utilization and system calls. Bachelor Thesis. Linköping University, 2015.

[10] M. Anand, E. B. Nightingale, and J. Flinn. Self-tuning wireless network power management. In Proceedings of the 9th Annual International Conference on Mobile Computing and Networking, MobiCom '03, pages 176-189. ACM, 2003.

[11] G. Anastasi, M. Conti, M. Di Francesco, and A. Passarella. Energy conservation in wireless sensor networks: A survey. Ad Hoc Networks, 7(3):537-568, Elsevier. May, 2009.

[12] S. Andersson. Energy consumption of 3G transmissions for instant messaging on mobile devices. Bachelor Thesis. Linköping University, 2013.

[13] AppDynamics. Application Performance Management and Monitoring, accessed 20th February, 2014.

http://www . appdynamics.com.

[14] T. Armstrong, O. Trescases, C. Amza, and E. de Lara. Efficient and transparent dynamic content updates for mobile clients. In Proceedings of the 4th International Conference on Mobile Systems, Applications and Services, MobiSys '06, pages 56-68. ACM, 2006.

[15] A. Artetxe, A. Beristain, and L. Kabongo. Activity classification using mobile phone based motion sensing and distributed computing. In 2nd KES International Conference on Innovation in Medicine and Healthcare, InMed 2014, volume 207, pages 1-10, IOS Press. 2014.

[16] A. Asadi and V. Mancuso. Energy efficient opportunistic uplink packet forwarding in hybrid wireless networks. In Proceedings of the 4 th International Conference on Future Energy Systems, e-Energy '13, pages 261-262. ACM, 2013.

[17] M. Asplund, A. Thomasson, E. J. Vergara, and S. Nadjm-Tehrani. Software-related energy footprint of a wireless broadband module. In Proceedings of the 9th ACM International Symposium on Mobility Management and Wireless Access, MobiWac '11. ACM, 2011.

[18] Atheros. Power consumption and energy efficiency comparisons of WLAN products. White paper, April 2004. Accessed 20th October 2013.

http://www . atheros.com/media/resource/resource_15_file2. pdf.

[19] P. K. Athivarapu, R. Bhagwan, S. Guha, V. Navda, R. Ramjee, D. Arora, V. N. Padmanabhan, and G. Varghese. Radiojockey: mining 
program execution to optimize cellular radio usage. In Proceedings of the 18th Annual International Conference on Mobile Computing and Networking, Mobicom '12, pages 101-112. ACM, 2012.

[20] K. Athukorala, E. Lagerspetz, M. von Kügelgen, A. Jylhä, A. J. Oliner, S. Tarkoma, and G. Jacucci. How carat affects user behavior: Implications for mobile battery awareness applications. In Proceedings of the 32nd Annual ACM Conference on Human Factors in Computing Systems, CHI '14, pages 1029-1038. ACM, 2014.

[21] G. Auer, V. Giannini, C. Desset, I. Godor, P. Skillermark, M. Olsson, M. Imran, D. Sabella, M. Gonzalez, O. Blume, and A. Fehske. How much energy is needed to run a wireless network? Wireless Communications, IEEE, 18(5):40-49, 2011.

[22] S. Baek and B. D. Choi. Analysis of Discontinuous Reception (DRX) with both downlink and uplink packet arrivals in 3GPP LTE. In Proceedings of the 6th International Conference on Queueing Theory and Network Applications, QTNA '11, pages 8-16. ACM, 2011.

[23] A. Balasubramanian, R. Mahajan, and A. Venkataramani. Augmenting mobile 3G using WiFi. In Proceedings of the 8th International Conference on Mobile Systems, Applications, and Services, MobiSys '10, pages 209-222. ACM, 2010.

[24] N. Balasubramanian, A. Balasubramanian, and A. Venkataramani. Energy consumption in mobile phones: a measurement study and implications for network applications. In Proceedings of the 9th ACM SIGCOMM conference on Internet measurement conference, IMC '09, pages 280-293. ACM, 2009.

[25] G. Banga, P. Druschel, and J. C. Mogul. Resource containers: A new facility for resource management in server systems. In Proceedings of the Third Symposium on Operating Systems Design and Implementation, OSDI '99, pages 45-58. USENIX Association, 1999.

[26] A. Barbuzzi, F. Ricciato, and G. Boggia. Discovering parameter setting in $3 \mathrm{G}$ networks via active measurements. Communications Letters, IEEE, 12(10):730-732, 2008.

[27] L. A. Barroso and U. Hölzle. The case for energy-proportional computing. Computer, IEEE, 40(12):33-37, Dec. 2007.

[28] F. Bellosa. The benefits of event: Driven energy accounting in powersensitive systems. In Proceedings of the 9th Workshop on ACM SIGOPS European Workshop: Beyond the PC: New Challenges for the Operating System, EW 9, pages 37-42. ACM, 2000. 
[29] G. Berg. Extension of EnergyBox for LTE Networks. Bachelor Thesis. Linköping University, 2016.

[30] V. Bernardo, M. Curado, and T. Braun. An IEEE 802.11 energy efficient mechanism for continuous media applications. Sustainable Computing: Informatics and Systems, 4(2):106 - 117, Elsevier. 2014.

[31] R. Bertran, Y. Becerra, D. Carrera, V. Beltran, M. Gonzalez Tallada, X. Martorell, J. Torres, and E. Ayguade. Accurate energy accounting for shared virtualized environments using PMC-based power modeling techniques. In 11th IEEE/ACM International Conference on Grid Computing (GRID), 2010, pages 1-8, Oct 2010.

[32] A. Bhojan, A. L. Akhihebbal, M. C. Chan, and R. K. Balan. ARIVU: making networked mobile games green. Mob. Netw. Appl., 17(1):2128, Feb. Springer-Verlag. 2012.

[33] A. P. Bianzino, M. Asplund, E. J. Vergara, and S. Nadjm-Tehrani. Cooperative proxies: Optimally trading energy and quality of service in mobile devices. Computer Networks, 75, Part A(0):297 - 312, Elsevier. 2014 .

[34] O. Blume, D. Zeller, A. Pascht, and K. Wuenstel. Green Future Mobile Networks. In ICT 2010, Networking Session ID: 3501 What does the future hold for mobile networks?, 2010, accessed 20th October 2013.

http://ec.europa.eu/digital-agenda/events/cf/ict2010/

document.cfm?doc_id=14742.

[35] F. Boccardi, R. Heath, A. Lozano, T. Marzetta, and P. Popovski. Five disruptive technology directions for 5G. Communications Magazine, IEEE, 52(2):74-80, February 2014.

[36] M. Böhmer, B. Hecht, J. Schöning, A. Krüger, and G. Bauer. Falling Asleep with Angry Birds, Facebook and Kindle: A Large Scale Study on Mobile Application Usage. In Proceedings of the 13th International Conference on Human Computer Interaction with Mobile Devices and Services, MobileHCI '11, pages 47-56. ACM, 2011.

[37] C. Bontu and E. Illidge. DRX mechanism for power saving in LTE. Communications Magazine, 47(6):48-55, IEEE, June 2009.

[38] O. Boyinbode, H. Le, A. Mbogho, M. Takizawa, and R. Poliah. A survey on clustering algorithms for wireless sensor networks. In 13th International Conference on Network-Based Information Systems (NBiS), 2010, pages 358-364, Sept. IEEE. 2010.

[39] L. Braun, A. Didebulidze, N. Kammenhuber, and G. Carle. Comparing and improving current packet capturing solutions based on 
commodity hardware. In Proceedings of the 10th ACM SIGCOMM Conference on Internet Measurement, IMC '10, pages 206-217. ACM, 2010.

[40] M. Calder and M. Marina. Batch scheduling of recurrent applications for energy savings on mobile phones. In 7th Annual IEEE Communications Society Conference on Sensor Mesh and Ad Hoc Communications and Networks (SECON), pages 1-3, June 2010.

[41] D. Camps-Mur, X. Pérez-Costa, and S. Sallent-Ribes. Designing energy efficient access points with Wi-Fi Direct. Computer Networks, 55(13), Elsevier. Sept. 2011.

[42] J. Castro, D. Gómez, and J. Tejada. Polynomial calculation of the shapley value based on sampling. Comput. Oper. Res., 36(5):17261730, Elsevier. May, 2009.

[43] D. Chalmers and M. Sloman. A survey of quality of service in mobile computing environments. Communications Surveys and Tutorials, 2(2):2-10, IEEE, 1999.

[44] R. Chandra, O. Fatemieh, P. Moinzadeh, C. A. Thekkath, and Y. Xie. End-to-end energy management of mobile devices. Technical Report MSR-TR-2013-69, Microsoft Research, July 2013.

[45] M.-H. Chen, C.-F. Chou, K.-H. Lee, and C.-Y. Chang. On cooperative energy-efficient $\mathrm{P} 2 \mathrm{P}$ live streaming system for mobile hotspots. In International Conference on Green Computing and Communications (GreenCom), 2013, IEEE. 2013.

[46] Y. Cheng, K. Chen, B. Zhang, C.-J. M. Liang, X. Jiang, and F. Zhao. Accurate real-time occupant energy-footprinting in commercial buildings. In Proceedings of the Fourth ACM Workshop on Embedded Sensing Systems for Energy-Efficiency in Buildings, BuildSys '12, pages 115-122. ACM, 2012.

[47] Z. Cheng, J. Caverlee, K. Lee, and D. Z. Sui. Exploring millions of footprints in location sharing services. In Proceedings of the Fifth International Conference on Weblogs and Social Media. AAAI, July 2011.

[48] J. Cho, Y. Woo, S. Kim, and E. Seo. A battery lifetime guarantee scheme for selective applications in smart mobile devices. IEEE Transactions on Consumer Electronics,, 60(1):155-163, February 2014.

[49] D. Chu, A. Kansal, J. Liu, and F. Zhao. Mobile apps: It's time to move up to condos. In Proceedings of the 13th USENIX Conference on Hot Topics in Operating Systems, HotOS'13, pages 16-16. USENIX Association, 2011. 
[50] Y. W. Chung. An improved energy saving scheme for instant messaging services. In Wireless Advanced, 2011, pages 278-282. IEEE, 2011.

[51] Y. W. Chung. Investigation of energy consumption of mobile station for instant messaging services. In Proceedings of the 2011 Tenth International Symposium on Autonomous Decentralized Systems, ISADS '11, pages 343-346, IEEE. 2011.

[52] V. Conitzer and T. Sandholm. Complexity of determining nonemptiness of the core. In Proceedings of the 4th ACM Conference on Electronic Commerce, EC '03, pages 230-231. ACM, 2003.

[53] V. Conitzer and T. Sandholm. Complexity of constructing solutions in the core based on synergies among coalitions. Artificial Intelligence, 170(6-7):607-619, Elsevier. 2006.

[54] I. Constandache, S. Gaonkar, M. Sayler, R. Choudhury, and L. Cox. EnLoc: Energy-Efficient Localization for Mobile Phones. In IEEE INFOCOM 2009, pages $2716-2720$, april 2009.

[55] A. Coté, A. Meyerson, and B. Tagiku. Energy-efficient mobile data transport via online multi-network packet scheduling. Sustainable Computing: Informatics and Systems, 1(3):196 - 212, 2011.

[56] E. Cuervo, A. Balasubramanian, D.-k. Cho, A. Wolman, S. Saroiu, R. Chandra, and P. Bahl. Maui: Making smartphones last longer with code offload. In Proceedings of the 8th International Conference on Mobile Systems, Applications, and Services, MobiSys '10, pages 49-62. ACM, 2010.

[57] E. Cuervo, A. Wolman, L. P. Cox, K. Lebeck, A. Razeen, S. Saroiu, and M. Musuvathi. Kahawai: High-quality mobile gaming using gpu offload. In Proceedings of the 13th Annual International Conference on Mobile Systems, Applications, and Services, MobiSys '15, pages 121-135. ACM, 2015.

[58] CurrentWidget. An Android widget that display the electric current usage of the device, accessed 20th February, 2014.

https://code.google.com/p/currentwidget/.

[59] S. Deng and H. Balakrishnan. Traffic-aware techniques to reduce 3G/LTE wireless energy consumption. In Proceedings of the 8th International Conference on Emerging Networking Experiments and Technologies, CoNEXT '12, pages 181-192. ACM, 2012.

[60] X. Deng and C. H. Papadimitriou. On the complexity of cooperative solution concepts. Math. Oper. Res., 19(2):257-266, May 1994. 
[61] N. Ding, D. Wagner, X. Chen, A. Pathak, Y. C. Hu, and A. Rice. Characterizing and modeling the impact of wireless signal strength on smartphone battery drain. In Proceedings of the ACM SIGMETRICS/International Conference on Measurement and Modeling of Computer Systems, SIGMETRICS '13, pages 29-40. ACM, 2013.

[62] M. Dong. Energy Accounting and Optimization for Mobile Systems. $\mathrm{PhD}$ thesis, Rice University, 2013.

[63] M. Dong, T. Lan, and L. Zhong. Rethink energy accounting with cooperative game theory. In Proceedings of the 20th Annual International Conference on Mobile Computing and Networking, MobiCom '14, pages 531-542. ACM, 2014.

[64] M. Dong and L. Zhong. Self-constructive high-rate system energy modeling for battery-powered mobile systems. In Proceedings of the 9th International Conference on Mobile Systems, Applications, and Services, MobiSys '11, pages 335-348. ACM, 2011.

[65] J. Drechsel. Selected topics in cooperative game theory. In Cooperative Lot Sizing Games in Supply Chains, volume 644 of Lecture Notes in Economics and Mathematical Systems, pages 5-39. Springer, 2010.

[66] J. Drechsel and A. Kimms. Computing core allocations in cooperative games with an application to cooperative procurement. International Journal of Production Economics, 128(1):310 - 321, Elsevier. 2010.

[67] M. Ekström, M. Bergblomma, M. Linden, M. Björkman, and M. Ekström. A Bluetooth radio energy consumption model for low-dutycycle applications. Instrumentation and Measurement, IEEE Transactions on, 61(3):609-617, 2012.

[68] M. El-Gendy, A. Bose, and K. Shin. Evolution of the internet QoS and support for soft real-time applications. Proceedings of the IEEE, 91(7):1086-1104, july 2003.

[69] C. Ellis. The case for higher-level power management. In Proceedings of the Seventh Workshop on Hot Topics in Operating Systems, 1999., pages 162-167, USENIX Association. 1999.

[70] S. Engevall, M. Göthe-Lundgren, and P. Värbrand. The traveling salesman game: An application ofcost allocation in a gas and oil company. Annals of Operations Research, 82(0):203-218, Springer. 1998.

[71] Ericsson Mobility Report, On the Pulse of the Networked Society. Ericsson, 2015. Accessed 26th May 2015.

http://www.ericsson. com/res/docs/2015/ericsson-mobilityreport-feb-2015-interim.pdf. 
[72] Ericsson Labs. Smartphone Traffic Impact on Battery and Networks, 2010. Accessed 26th May 2015.

http://www. ericsson.com/research-blog/uncategorized/ smartphone-traffic-impact-battery-networks/.

[73] M. Etoh, T. Ohya, and Y. Nakayama. Energy consumption issues on mobile network systems. In Proceedings of the 2008 International Symposium on Applications and the Internet, pages 365-368, IEEE. 2008.

[74] U. Faigle, W. Kern, and J. Kuipers. Computing the nucleolus of mincost spanning tree games is NP-hard. Int. J. Game Theory, 27(3):443450, Physica-Verlag, Dec. 1998.

[75] H. Falaki, R. Mahajan, S. Kandula, D. Lymberopoulos, R. Govindan, and D. Estrin. Diversity in smartphone usage. In Proceedings of the 8th International Conference on Mobile Systems, Applications, and Services, MobiSys '10, pages 179-194. ACM, 2010.

[76] S. S. Fatima, M. Wooldridge, and N. R. Jennings. A linear approximation method for the shapley value. Artif. Intell., 172(14):1673-1699, Sept. Elsevier. 2008.

[77] L. Feeney and M. Nilsson. Investigating the energy consumption of a wireless network interface in an ad hoc networking environment. In Proceedings of the Twentieth Annual Joint Conference of the IEEE Computer and Communications Societies. INFOCOM 2001, pages 1548-1557 vol.3, 2001.

[78] M. Fiestras-Janeiro, I. García-Jurado, and M. Mosquera. Cooperative games and cost allocation problems. TOP, 19(1):1-22, SpringerVerlag. 2011.

[79] J. Flinn and M. Satyanarayanan. Powerscope: A tool for profiling the energy usage of mobile applications. In Proceedings of the Second IEEE Workshop on Mobile Computer Systems and Applications, WMCSA '99, pages 2-11. IEEE Computer Society, 1999.

[80] J. Flinn and M. Satyanarayanan. Managing battery lifetime with energy-aware adaptation. ACM Trans. Comput. Syst., 22(2):137-179, May 2004.

[81] J. Flinn and M. Satyanarayanan. Energy-aware adaptation for mobile applications. SIGOPS Oper. Syst. Rev., 33(5):48-63, ACM. Dec. 1999.

[82] S. Foll, K. Herrmann, and K. Rothermel. Energy-Efficient Update Protocols for Mobile User Context. In IEEE 26th International Conference on Advanced Information Networking and Applications (AINA), 2012, pages $120-127$, march 2012. 
[83] R. Fonseca, P. Dutta, P. Levis, and I. Stoica. Quanto: Tracking energy in networked embedded systems. In Proceedings of the 8th USENIX Conference on Operating Systems Design and Implementation, OSDI'08, pages 323-338. USENIX Association, 2008.

[84] R. Friedman, A. Kogan, and Y. Krivolapov. On power and throughput tradeoffs of WiFi and Bluetooth in smartphones. IEEE Transactions on Mobile Computing, 12(7):1363-1376, July 2013.

[85] F. Ganji, L. Budzisz, F. G. Debele, N. Li, M. Meo, M. Ricca, Y. Zhang, and A. Wolisz. Greening campus WLANs: Energy-relevant usage and mobility patterns. Computer Networks, 78:164 - 181, Elsevier. 2015.

[86] J. González-Díaz and E. Sánchez-Rodríguez. Understanding the coincidence of allocation rules: symmetry and orthogonality in tu-games. International Journal of Game Theory, 43(4):821-843, Springer. 2014.

[87] J. Green Olander. Optimizing Communication Energy Efficiency for a Multimedia Application. Master Thesis. Linköping University, 2016.

[88] R. E. Grinter, L. Palen, and M. Eldridge. Chatting with teenagers: Considering the place of chat technologies in teen life. ACM Trans. Comput.-Hum. Interact., 13(4):423-447, Dec. 2006.

[89] The Mobile Economy. GSMA., 2015. Accessed 26th May 2015. http://www.gsmamobileeconomy.com/GSMA_Global_Mobile_ Economy_Report_2015.pdf.

[90] L. Guo, X. Ding, H. Wang, Q. Li, S. Chen, and X. Zhang. Cooperative relay service in a wireless LAN. IEEE Journal on Selected Areas in Communications, 25(2):355-368, February 2007.

[91] H. Hall and C. Luckey. 3G Transmission Energy Savings through Adaptive Traffic Shaping Policies. Bachelor Thesis. Linköping University, 2014.

[92] D. Halperin, B. Greenstein, A. Sheth, and D. Wetherall. Demystifying 802.11n power consumption. In Proceedings of the 2010 International Conference on Power Aware Computing and Systems, HotPower'10, pages 1-5. USENIX Association, 2010.

[93] B. Han, P. Hui, V. Kumar, M. Marathe, J. Shao, and A. Srinivasan. Mobile data offloading through opportunistic communications and social participation. IEEE Transactions on Mobile Computing,, 11(5):821-834, May 2012.

[94] H. Han, Y. Liu, G. Shen, Y. Zhang, and Q. Li. DozyAP: power-efficient Wi-Fi tethering. In Proceedings of the 10th international conference on Mobile systems, applications, and services, MobiSys '12, pages 421434. ACM, 2012. 
[95] E. Harjula, O. Kassinen, and M. Ylianttila. Energy consumption model for mobile devices in $3 \mathrm{G}$ and WLAN networks. In IEEE Consumer Communications and Networking Conference (CCNC), pages 532-537, 2012.

[96] Z. Hasan, H. Boostanimehr, and V. Bhargava. Green cellular networks: A survey, some research issues and challenges. Communications Surveys Tutorials, 13(4):524-540, IEEE, 2011.

[97] S. Hay and A. Rice. The case for apportionment. In Proceedings of the First ACM Workshop on Embedded Sensing Systems for EnergyEfficiency in Buildings, BuildSys '09, pages 13-18. ACM, 2009.

[98] W. R. Heinzelman, A. Chandrakasan, and H. Balakrishnan. Energyefficient communication protocol for wireless microsensor networks. In Proceedings of the 33rd Hawaii International Conference on System Sciences, HICSS '00. Vol. 8. IEEE, 2000.

[99] B. D. Higgins, A. Reda, T. Alperovich, J. Flinn, T. J. Giuli, B. Noble, and D. Watson. Intentional networking: opportunistic exploitation of mobile network diversity. In Proceedings of the Sixteenth Annual International Conference on Mobile Computing and Networking, MobiCom '10, pages 73-84. ACM, 2010.

[100] T. Hoífeld and A. Binzenhöfer. Analysis of Skype VoIP traffic in UMTS: End-to-end QoS and QoE measurements. Computer Networks, 52(3):650-666, Elsevier. Feb. 2008.

[101] R. Holm. Energy-Efficient Mobile Communication with Cached Signal Maps. Bachelor Thesis. Linköping University, 2016.

[102] H. Holma and A. Toskala. WCDMA for UMTS: HSPA Evolution and LTE. Wiley Online Library: Books. John Wiley \& Sons, Ltd., 2010.

[103] M. Hoque, M. Siekkinen, and J. Nurminen. Energy efficient multimedia streaming to mobile devices - a survey. Communications Surveys Tutorials, 16(1):579-597, IEEE, 2014.

[104] M. A. Hoque, M. Siekkinen, K. N. Khan, Y. Xiao, and S. Tarkoma. Modeling, profiling, and debugging the energy consumption of mobile devices. ACM Comput. Surv., 48(3):39:1-39:40, Dec. 2015.

[105] C.-Y. Huang, C.-M. Chen, S.-P. Yu, S.-Y. Hsu, and C.-H. Lin. Accelerate in-line packet processing using fast queue. In Proceedings of IEEE TENCON 2010, Nov 2010.

[106] J. Huang, F. Qian, A. Gerber, Z. M. Mao, S. Sen, and O. Spatscheck. A close examination of performance and power characteristics of $4 \mathrm{~g}$ lte networks. In Proceedings of the 10th International Conference on 
Mobile Systems, Applications, and Services, MobiSys '12, pages 225238. ACM, 2012.

[107] J. Huang, F. Qian, Z. M. Mao, S. Sen, and O. Spatscheck. Screenoff traffic characterization and optimization in $3 \mathrm{G} / 4 \mathrm{G}$ networks. In Proceedings of the 2012 ACM Internet Measurement Conference, IMC '12, pages 357-364. ACM, 2012.

[108] I. Humar, X. Ge, L. Xiang, M. Jo, M. Chen, and J. Zhang. Rethinking energy efficiency models of cellular networks with embodied energy. IEEE Network, 25(2):40-49, Mar. 2011.

[109] In a Near Tie, Apple Closes the Gap on Samsung in the Fourth Quarter as Worldwide Smartphone Shipments Top 1.3 Billion for 2014. IDC, 2015. Accessed 26th May 2015.

http: //www . idc . com/getdoc . j sp? container Id=prUS25407215.

[110] IEEE. 802.21 Media Independent Handover http://www.ieee802.org/21/ accessed 20th October 2013.

[111] E. Isaacs, A. Walendowski, S. Whittaker, D. J. Schiano, and C. Kamm. The character, functions, and styles of instant messaging in the workplace. In Proceedings of the 2002 ACM Conference on Computer Supported Cooperative Work, pages 11-20. ACM, 2002.

[112] A. P. Jardosh, K. Papagiannaki, E. M. Belding, K. Almeroth, G. Iannaccone, and B. Vinnakota. Green WLANs: On-demand WLAN infrastructures. Mobile Networks and Applications, 14(6):798-814, Springer. 2009.

[113] M. Jones, P. Leach, R. Draves, and I. Barrera, J.S. Modular real-time resource management in the Rialto operating system. In Fifth Workshop on Hot Topics in Operating Systems, 1995. (HotOS-V), pages 12-17. USENIX Association, May 1995.

[114] A. Kansal, F. Zhao, J. Liu, N. Kothari, and A. A. Bhattacharya. Virtual machine power metering and provisioning. In Proceedings of the 1st ACM Symposium on Cloud Computing, SoCC '10, pages 39-50. ACM, 2010.

[115] Y. Kawahara, H. Kurasawa, and H. Morikawa. Recognizing user context using mobile handsets with acceleration sensors. In IEEE International Conference on Portable Information Devices, PORTABLE0\%., pages 1-5, May 2007.

[116] S. Kellner. Flexible online energy accounting in TinyOS. In Proceedings of the 4 th International Workshop on Real-World Wireless Sensor Networks (RealWSN'10), volume 6511 of Lecture Notes in Computer Science, pages 62-73. Springer, Dec. 2010. 
[117] K. Keshav, V. Indukuri, and P. Venkataram. Energy efficient scheduling in $4 \mathrm{G}$ smart phones for mobile hotspot application. In National Conference on Communications (NCC), pages 1-5. IEEE, Feb. 2012.

[118] M. J. Kim, S. W. Son, and B. H. Rhee. A new approach network selection with MIH between WLAN and WMAN. In Fourth International Conference on Computer Sciences and Convergence Information Technology, ICCIT '09, pages 751-755, IEEE, Nov. 2009.

[119] R. Y. Kim and S. Mohanty. Advanced power management techniques in next-generation wireless networks. Communications Magazine, IEEE, 48(5):94-102, May 2010.

[120] M. Kjærgaard. Location-based services on mobile phones: minimizing power consumption. Pervasive Computing, IEEE, 11(1):67 -73, 2012.

[121] M. Kjærgaard and H. Blunck. Unsupervised power profiling for mobile devices. In Mobile and Ubiquitous Systems: Computing, Networking, and Services, Lecture Notes of the Institute for Computer Sciences, Social Informatics and Telecommunications Engineering, pages 138149. Springer, 2012.

[122] M. B. Kjærgaard, J. Langdal, T. Godsk, and T. Toftkjær. EnTracked: Energy-Efficient Robust Position Tracking for Mobile Devices. In Proceedings of the 7th international conference on Mobile systems, applications, and services, MobiSys '09, pages 221-234. ACM, 2009.

[123] V. Könönen and P. Paakkonen. Optimizing power consumption of always-on applications based on timer alignment. In Third International Conference on Communication Systems and Networks (COMSNETS), pages 1-8. IEEE, Jan. 2011.

[124] R. Krashinsky and H. Balakrishnan. Minimizing energy for wireless web access with bounded slowdown. In Proceedings of the 8th Annual International Conference on Mobile Computing and Networking, MobiCom '02, pages 119-130. ACM, 2002.

[125] H. A. Lagar-Cavilla, K. Joshi, A. Varshavsky, J. Bickford, and D. Parra. Traffic backfilling: subsidizing lunch for delay-tolerant applications in umts networks. SIGOPS Operating Systems Review, 45(3):77-81, ACM. Jan. 2012.

[126] C.-C. Lee, J.-H. Yeh, and J.-C. Chen. Impact of inactivity timer on energy consumption in WCDMA and cdma2000. In Wireless Telecommunications Symposium, pages 15-24, IEEE. 2004.

[127] E. A. Lee. Modeling concurrent real-time processes using discrete events. Ann. Softw. Eng., 7(1-4):25-45, J. C. Baltzer AG, Science Publishers, June 1999. 
[128] K. Lee, D. Chu, E. Cuervo, J. Kopf, Y. Degtyarev, S. Grizan, A. Wolman, and J. Flinn. Outatime: Using speculation to enable low-latency continuous interaction for mobile cloud gaming. In Proceedings of the 13th Annual International Conference on Mobile Systems, Applications, and Services, MobiSys '15, pages 151-165. ACM, 2015.

[129] J. Lemaire. An application of game theory: Cost allocation. ASTIN Bulletin, 14:61-81, 41984.

[130] J. Leskovec and E. Horvitz. Planetary-scale views on a large instantmessaging network. In Proceedings of the 17th International Conference on World Wide Web, pages 915-924. ACM, 2008.

[131] B. Leslie, P. Chubb, N. Fitzroy-dale, S. Götz, C. Gray, L. Macpherson, D. Potts, Y. Shen, K. Elphinstone, and G. Heiser. User-level device drivers: Achieved performance. Journal of Computer Science and Technology, 20(5):654-664, Springer. 2005.

[132] C.-Y. Li, C. Peng, S. Lu, and X. Wang. Energy-based rate adaptation for $802.11 \mathrm{n}$. In Proceedings of the 18th Annual International Conference on Mobile Computing and Networking, Mobicom '12, pages 341-352. ACM, 2012.

[133] Y. Li, H. Chen, and W. Shi. Power behavior analysis of mobile applications using Bugu. Sustainable Computing: Informatics and Systems, 4(3):183 - 195, Elsevier. 2014.

[134] F. Liers, C. Burkhardt, and A. Mitschele-Thiel. Static RRC timeouts for various traffic scenarios. In Proceedings of the 18th International Symposium on Personal, Indoor and Mobile Radio Communications, PIMRC 2007, pages 1-5, IEEE. 2007.

[135] F. Liers and A. Mitschele-Thiel. UMTS data capacity improvements employing dynamic RRC timeouts. In Proceedings of the 16th International Symposium on Personal, Indoor and Mobile Radio Communications, PIMRC 2005, pages 2186-2190 Vol. 4, IEEE. 2005.

[136] K. Lin, A. Kansal, D. Lymberopoulos, and F. Zhao. Energy-Accuracy Trade-off for Continuous Mobile Device Location. In Proceedings of the 8th international conference on Mobile systems, applications, and services, MobiSys '10, pages 285-298. ACM, 2010.

[137] H. Liu, Y. Zhang, and Y. Zhou. TailTheft: leveraging the wasted time for saving energy in cellular communications. In Proceedings of the Sixth International Workshop on MobiArch, MobiArch '11, pages 31-36. ACM, 2011. 
[138] H. Luo, R. Ramjee, P. Sinha, L. E. Li, and S. Lu. UCAN: a unified cellular and ad-hoc network architecture. In Proceedings of the 9th Annual International Conference on Mobile Computing and Networking, MobiCom '03, pages 353-367. ACM, 2003.

[139] J. Manweiler, S. Agarwal, M. Zhang, R. Roy Choudhury, and P. Bahl. Switchboard: A matchmaking system for multiplayer mobile games. In Proceedings of the 9th International Conference on Mobile Systems, Applications, and Services, MobiSys '11, pages 71-84. ACM, 2011.

[140] J. Manweiler and R. R. Choudhury. Avoiding the rush hours: WiFi energy management via traffic isolation. IEEE Transactions on Mobile Computing, 11(5):739-752, 2012.

[141] J. Marian Santos, S. Nadjm-Tehrani, and A. Bianzino. Monkey Gamer: Automatic profiling of Android games. In 6th International Conference on Mobile Computing, Applications and Services (Mobi$C A S E)$, pages 125-132. IEEE, Nov 2014.

[142] M. Marsan, L. Chiaraviglio, D. Ciullo, and M. Meo. Optimal energy savings in cellular access networks. In International Conference on Communications. ICC Workshops, pages 1-5, IEEE. June 2009.

[143] M. A. Marsan and M. Meo. Energy efficient wireless internet access with cooperative cellular networks. Computer Networks, 55(2):386398, Elsevier. 2011.

[144] M. Maschler, B. Peleg, and L. S. Shapley. Geometric properties of the kernel, nucleolus, and related solution concepts. Mathematics of Operations Research, 4(4):303-338, INFORMS. 1979.

[145] M. Maschler, J. Potters, and S. Tijs. The general nucleolus and the reduced game property. International Journal of Game Theory, 21(1):85-106, Physica-Verlag. 1992.

[146] S. Masuya and M. Inuiguchi. Toward the theory of cooperative games under incomplete information. In Proceedings of the 6th International Conference on Modeling Decisions for Artificial Intelligence, MDAI '09, pages 102-113. Springer-Verlag, 2009.

[147] R. A. McCain. Value Solutions in Cooperative Games. World Scientific Publishing Company, 2013.

[148] N. Megiddo. Computational complexity of the game theory approach to cost allocation for a tree. Mathematics of Operations Research, 3(3):189-196, INFORMS. 1978.

[149] L.-S. Meng, D.-S. Shiu, P.-C. Yeh, K.-C. Chen, and H.-Y. Lo. Low power consumption solutions for mobile instant messaging. IEEE Transactions on Mobile Computing, 11(6):896-904, June 2012. 
[150] Y. Mihov, K. Kassev, and B. Tsankov. Analysis and performance evaluation of the DRX mechanism for power saving in LTE. In IEEE 26th Convention of Electrical and Electronics Engineers in Israel (IEEEI), 2010, pages 520-524, Nov 2010.

[151] V. Misra, S. Ioannidis, A. Chaintreau, and L. Massoulié. Incentivizing peer-assisted services: A fluid shapley value approach. In Proceedings of the ACM SIGMETRICS International Conference on Measurement and Modeling of Computer Systems, SIGMETRICS '10, pages 215226. ACM, 2010.

[152] R. Mittal, A. Kansal, and R. Chandra. Empowering developers to estimate app energy consumption. In Proceedings of the 18th Annual International Conference on Mobile Computing and Networking, Mobicom '12, pages 317-328. ACM, 2012.

[153] E. Molina and J. Tejada. The least square nucleolus is a general nucleolus. International Journal of Game Theory, 29(1):139-142, SpringerVerlag. 2000.

[154] Monsoon Solutions Inc., Power Monitor, accessed 20th February, 2014.

http://www .msoon. com/LabEquipment/PowerMonitor/.

[155] U. Moreno. Energy Consumption Studies for 3G Traffic Consolidation on Android using WiFi and Bluetooth. Master Thesis. Linköping University, 2014.

[156] S. Moretti and F. Patrone. Transversality of the Shapley value. TOP, 16(1):1-41, Springer-Verlag. 2008.

[157] Message Queueing Telemetry Transport specification v3.1, accessed 20th October 2013.

http://public.dhe.ibm.com/software/dw/webservices/ws$\mathrm{mqtt} / \mathrm{mqtt}-\mathrm{v} 3 \mathrm{r} 1 . \mathrm{html}$.

[158] R. B. Myerson. Game Theory: Analysis of Conflict. Harvard University Press, 1991.

[159] M. Nam, N. Choi, Y. Seok, and Y. Choi. WISE: energy-efficient interface selection on vertical handoff between $3 \mathrm{G}$ networks and WLANs. In Proceedings of the 15th IEEE International Symposium on Personal, Indoor and Mobile Radio Communications, PIMRC 2004, pages 692698 Vol.1, 2004.

[160] National Instruments. NI USB-6255, accessed 20th February, 2014. http://sine.ni.com/nips/cds/view/p/lang/sv/nid/209157. 
[161] R. Neugebauer and D. McAuley. Energy Is Just Another Resource: Energy Accounting and Energy Pricing in the Nemesis OS. In Proceedings of the Eighth Workshop on Hot Topics in Operating Systems, HOTOS '01. IEEE, 2001.

[162] New Relic. Mobile Application Monitoring: Cloud Based App Monitoring, accessed 20th February, 2014.

http://newrelic.com/mobile-monitoring.

[163] N. Nguyen, Y. Wang, X. Liu, R. Zheng, and Z. Han. A nonparametric bayesian approach for opportunistic data transfer in cellular networks. In Wireless Algorithms, Systems, and Applications, Lecture Notes in Computer Science, pages 88-99 Vol. 7405. Springer, 2012.

[164] S. Nilsson and J. Eriksson. Estimating Application Energy Consumption Through Packet Trace Analysis. Bachelor Thesis. Linköping University, 2014.

[165] Z. Niu, Y. Wu, J. Gong, and Z. Yang. Cell zooming for cost-efficient green cellular networks. Communications Magazine, IEEE, 48(11):7479, November 2010.

[166] Nokia. Nokia Energy Profiler, accessed 20th February, 2014. http://store.ovi.com/content/73969.

[167] I. Obermiller and S. Bayless. Legends of Descent: Analytics in an Adsupported Windows Phone Game. In Proceedings of the First ACM International Workshop on Mobile Gaming, MobileGames '12, pages 1-6. ACM, 2012.

[168] A. J. Oliner, A. P. Iyer, I. Stoica, E. Lagerspetz, and S. Tarkoma. Carat: Collaborative energy diagnosis for mobile devices. In Proceedings of the 11th ACM Conference on Embedded Networked Sensor Systems, SenSys '13, pages 10:1-10:14. ACM, 2013.

[169] T. Oliveira, E. Ursini, and V. Timoteo. Simulation inspired model for energy consumption in $3 \mathrm{G}$ always-on mobiles. In IEEE 2nd National Conference on Telecommunications (CONATEL), pages 1-7, 2011.

[170] E. A. Oliver and S. Keshav. An empirical approach to smartphone energy level prediction. In Proceedings of the 13th International Conference on Ubiquitous Computing, UbiComp '11, pages 345-354. ACM, 2011.

[171] R. Palit, K. Naik, and A. Singh. Impact of packet aggregation on energy consumption in smartphones. In 7th International Wireless Communications and Mobile Computing Conference, IWCMC 2011, pages 589-594, IEEE. July 2011. 
[172] A. Papathanasiou and M. Scott. Energy efficiency through burstiness. In Fifth IEEE Workshop on Mobile Computing Systems and Applications, 2003, pages 44-53, Oct 2003.

[173] A. Pathak, Y. C. Hu, and M. Zhang. Where is the energy spent inside my app?: Fine grained energy accounting on smartphones with Eprof. In Proceedings of the 7th ACM European Conference on Computer Systems, EuroSys '12, pages 29-42, 2012.

[174] A. Patro, S. Rayanchu, M. Griepentrog, Y. Ma, and S. Banerjee. The Anatomy of a Large Mobile Massively Multiplayer Online Game. SIGCOMM Comput. Commun. Rev., 42(4):479-484, ACM, Sept. 2012.

[175] U. Paul, A. Subramanian, M. Buddhikot, and S. Das. Understanding traffic dynamics in cellular data networks. In Proceedings of the 30th IEEE International Conference on Computer Communications, INFOCOM 2011, pages 882-890, 2011.

[176] M. Pedersen, F. Fitzek, G. P. Perrucci, and T. Larsen. Energy and link measurements for mobile phones using IEEE $802.11 \mathrm{~b} / \mathrm{g}$. In Proceedings of the 6th International Symposium on Modeling and Optimization in Mobile, Ad Hoc, and Wireless Networks and Workshops, WiOPT 2008, pages 36-42, IEEE. April 2008.

[177] P. Perala, A. Barbuzzi, G. Boggia, and K. Pentikousis. Theory and practice of RRC state transitions in UMTS networks. In GLOBECOM Workshops, 2009, pages 1-6, IEEE. 2009.

[178] T. Pering, Y. Agarwal, R. Gupta, and R. Want. CoolSpots: Reducing the power consumption of wireless mobile devices with multiple radio interfaces. In Proceedings of the 4th International Conference on Mobile Systems, Applications and Services, MobiSys '06, pages 220-232. ACM, 2006.

[179] G. Perrucci, F. Fitzek, G. Sasso, and M. Katz. Energy saving strategies for mobile devices using wake-up signals. In 4th International Mobile Multimedia Communications Conference, MobiMedia 2008. ICTS/ACM, July 2008.

[180] G. P. Perrucci, P. Anggraeni, S. Wardana, F. H. P. Fitzek, and M. Katz. Bio-inspired energy-aware protocol design for cooperative wireless networks. International Journal of Autonomous and Adaptive Communications Systems (IJAACS), 4(2):164-179, Inderscience, Apr. 2011.

[181] G. P. Perrucci, F. Fitzek, G. Sasso, W. Kellerer, and J. Widmer. On the impact of $2 \mathrm{G}$ and $3 \mathrm{G}$ network usage for mobile phones' battery life. In European Wireless 2009, IEEE. May 2009. 
[182] G. P. Perrucci, F. H. P. Fitzek, Q. Zhang, and M. D. Katz. Cooperative mobile web browsing. EURASIP Journal on Wireless Communications and Networking, 2009, April 2009.

[183] G. P. Perrucci, M. V. Pedersen, T. K. Madsen, and F. H. P. Fitzek. Energy evaluation for Bluetooth link layer packet selection scheme. In European Wireless 2009, IEEE. 2009.

[184] J. E. Petersen, V. Shunturov, K. Janda, G. Platt, and K. Weinberger. Dormitory residents reduce electricity consumption when exposed to real-time visual feedback and incentives. International Journal of Sustainability in Higher Education, 8:16-33, 2007.

[185] R. Polis. EnergyBox: Tool improvement and GUI. Bachelor Thesis. Linköping University, 2014.

[186] L. Polkowski and B. Araszkiewicz. A rough set approach to estimating the game value and the shapley value from data. In International Workshop on Rough Sets in Knowledge Discovery and Soft Computing (Satellite Event for ETAPS 2003), volume 82, pages 219 - 227, Elsevier. 2003.

[187] J.D. Power and Associates Reports: Smartphone Battery Life has Become a Significant Drain on Customer Satisfaction and Loyalty. PR Newswire, 2012. Accessed 26th May 2015.

http://www . prnewswire.com/news-releases/jd-power-andassociates-reports-smartphone-battery-life-has-become-asignificant-drain-on-customer-satisfaction-and-loyalty142765065.html.

[188] M. Prihodko. Energy consumption in location sharing protocols for Android applications. Master Thesis. Linköping University, 2012.

[189] I. Prochkova, V. Singh, and J. K. Nurminen. Energy Cost of Advertisements in Mobile Games on the Android Platform. In Proceedings of the 2012 Sixth International Conference on Next Generation Mobile Applications, Services and Technologies, NGMAST '12, pages 147152. IEEE, 2012.

[190] J. Puerto and F. Perea. Finding the nucleolus of any n-person cooperative game by a single linear program. Computers and Operations Research, 40(10):2308 - 2313, Elsevier. 2013.

[191] I. Puustinen and J. Nurminen. The effect of unwanted internet traffic on cellular phone energy consumption. In 4th IFIP International Conference on New Technologies, Mobility and Security, NTMS 2011, pages 1-5, IEEE. Feb. 2011. 
[192] A. J. Pyles, X. Qi, G. Zhou, M. Keally, and X. Liu. SAPSM: Smart adaptive 802.11 PSM for smartphones. In Proceedings of the 14th International Conference on Ubiquitous Computing, UbiComp '12. ACM, 2012.

[193] A. J. Pyles, Z. Ren, G. Zhou, and X. Liu. SiFi: Exploiting VoIP silence for WiFi energy savings in smart phones. In Proceedings of the 13th International Conference on Ubiquitous Computing, UbiComp '11, pages 325-334. ACM, 2011.

[194] F. Qian, J. Huang, J. Erman, Z. M. Mao, S. Sen, and O. Spatscheck. How to reduce smartphone traffic volume by $30 \%$ ? In Proceedings of the 14th International Conference on Passive and Active Measurement, pages 42-52. Springer-Verlag, 2013.

[195] F. Qian, Z. Wang, Y. Gao, J. Huang, A. Gerber, Z. Mao, S. Sen, and O. Spatscheck. Periodic transfers in mobile applications: networkwide origin, impact, and optimization. In Proceedings of the 21st International Conference on World Wide Web, WWW'12, pages 51-60. ACM, 2012.

[196] F. Qian, Z. Wang, A. Gerber, Z. Mao, S. Sen, and O. Spatscheck. TOP: Tail optimization protocol for cellular radio resource allocation. In 18th International Conference on Network Protocols (ICNP), 2010, pages 285-294, IEEE. Oct. 2010.

[197] F. Qian, Z. Wang, A. Gerber, Z. Mao, S. Sen, and O. Spatscheck. Profiling resource usage for mobile applications: A cross-layer approach. In Proceedings of the 9th International Conference on Mobile Systems, Applications, and Services, MobiSys '11, pages 321-334. ACM, 2011.

[198] F. Qian, Z. Wang, A. Gerber, Z. M. Mao, S. Sen, and O. Spatscheck. Characterizing radio resource allocation for $3 \mathrm{G}$ networks. In Proceedings of the 10th ACM SIGCOMM Conference on Internet Measurement, IMC '10, pages 137-150. ACM, 2010.

[199] L. Qian, E. W. W. Chan, P. P. C. Lee, and C. He. Characterization of 3G Control-Plane Signaling Overhead from a Data-Plane Perspective. In Proceedings of the 15th ACM International Conference on Modeling, Analysis and Simulation of Wireless and Mobile Systems, MSWiM '12. ACM, 2012.

[200] Qualcomm. Snapdragon Development Platform, accessed 20th February, 2014.

http://www . qualcomm. com/snapdragon/tools/developmentplatform.

[201] Trepn Profiler. Qualcomm, accessed 20th February, 2014. https://developer .qualcomm.com/mobile-development/ development-devices/trepn-profiler. 
[202] M.-R. Ra, J. Paek, A. B. Sharma, R. Govindan, M. H. Krieger, and M. J. Neely. Energy-delay tradeoffs in smartphone applications. In Proceedings of the 8th International Conference on Mobile Systems, Applications, and Services, MobiSys '10, pages 255-270. ACM, 2010.

[203] Radio Link Control protocol, 3GPP specification 25.322, accessed 20th October 2013.

http: //www . 3gpp.org/ftp/Specs/html-info/25322.htm.

[204] A. Rahmati, C. Shepard, C. Tossell, L. Zhong, P. Kortum, A. Nicoara, and J. Singh. Seamless TCP migration on smartphones without network support. IEEE Transactions on Mobile Computing, (99), 2013.

[205] A. Rahmati and L. Zhong. Context-based network estimation for energy-efficient ubiquitous wireless connectivity. IEEE Transactions on Mobile Computing, 10(1):54-66, 2011.

[206] P. Ranganathan. Recipe for efficiency: Principles of power-aware computing. Commun., 53(4):60-67, ACM, Apr. 2010.

[207] A. Reinhardt, P. Baumann, D. Burgstahler, M. Hollick, H. Chonov, M. Werner, and R. Steinmetz. On the accuracy of appliance identification based on distributed load metering data. In Sustainable Internet and ICT for Sustainability (SustainIT), 2012, pages 1-9, IFIP. Oct 2012.

[208] A. Rice and S. Hay. Measuring mobile phone energy consumption for 802.11 wireless networking. Pervasive Mobile Computing, 6(6):593606, Elsevier. Dec. 2010.

[209] J. Rodriguez, M. Tsagaropoulos, I. Politis, S. Kotsopoulos, and T. Dagiuklas. A middleware architecture supporting seamless and secure multimedia services across an intertechnology radio access network. Wireless Communications, IEEE, 16(5):24-31, October 2009.

[210] T\&M Solution. Rohde \& Schwarz, accessed 20th October 2013. http://www .rohde-schwarz.com/en/applications/optimizethe-quality-of-experience-of-mobile-devices-applicationcard_56279-35727.html.

[211] A. P. Rosenbusch. Fairness Considerations in Cooperative Games. PhD thesis, TU Darmstadt / Mathematik, May 2011.

[212] A. Roy, S. M. Rumble, R. Stutsman, P. Levis, D. Mazières, and N. Zeldovich. Energy Management in Mobile Devices with the Cinder Operating System. In Proceedings of the Sixth Conference on Computer Systems, EuroSys '11, pages 139-152. ACM, 2011. 
[213] E. Rozner, V. Navda, R. Ramjee, and S. Rayanchu. NAPman: Network-assisted power management for WiFi devices. In Proceedings of the 8th International Conference on Mobile Systems, Applications, and Services, MobiSys '10, pages 91-106. ACM, 2010.

[214] L. M. Ruiz, F. Valenciano, and J. M. Zarzuelo. The Least Square Prenucleolus and the Least Square Nucleolus. Two values for TU Games based on the excess vector. International Journal of Game theory, 25:113-134, Physica-Verlag. 1996.

[215] S. M. Rumble, R. Stutsman, P. Levis, D. Mazières, and N. Zeldovich. Apprehending joule thieves with cinder. In Proceedings of the 1st ACM Workshop on Networking, Systems, and Applications for Mobile Handhelds, MobiHeld '09, pages 49-54. ACM, 2009.

[216] S. Ryffel. LEA2P the linux energy attribution and accounting platform. Master's thesis, Swiss Federal Institute of Technology, 2009.

[217] S. Ryffel, T. Stathopoulos, D. McIntire, W. Kaiser, and L. Thiele. Accurate energy attribution and accounting for multi-core systems. Technical report, Center for Embedded Network Sensing, University of California, Los Angeles, September 2009.

[218] M. Saha, S. Thakur, A. Singh, and Y. Agarwal. Energylens: Combining smartphones with electricity meter for accurate activity detection and user annotation. In Proceedings of the 5th International Conference on Future Energy Systems, e-Energy '14, pages 289-300. ACM, 2014.

[219] J. Sanjuan. 3G energy-efficient packet handling kernel module for Android. Master Thesis. Linköping University, 2012.

[220] D. Schmeidler. The nucleolus of a characteristic function game. SIAM Journal of Applied Mathematics, pages 1163-1170, Society for Industrial and Applied Mathematics. 1969.

[221] F. Schneider, J. Wallerich, and A. Feldmann. Packet capture in 10gigabit ethernet environments using contemporary commodity hardware. In Passive and Active Network Measurement, volume 4427 of Lecture Notes in Computer Science, pages 207-217. Springer, 2007.

[222] S. Schrittwieser, P. Frühwirt, P. Kieseberg, M. Leithner, M. Mulazzani, M. Huber, and E. R. Weippl. Guess who's texting you? evaluating the security of smartphone messaging applications. In 19th Annual Network 8 Distributed System Security Symposium (NDSS), The Internet Society (ISOC). 2012.

[223] A. Schulman, V. Navda, R. Ramjee, N. Spring, P. Deshpande, C. Grunewald, K. Jain, and V. N. Padmanabhan. Bartendr: a practical approach to energy-aware cellular data scheduling. In Proceedings 
of the Sixteenth Annual International Conference on Mobile Computing and Networking, MobiCom '10, pages 85-96. ACM, 2010.

[224] M. Sereno. Cooperative game theory framework for energy efficient policies in wireless networks. In Proceedings of the 3rd International Conference on Future Energy Systems: Where Energy, Computing and Communication Meet, e-Energy '12, pages 17:1-17:9. ACM, 2012.

[225] M. Z. Shafiq, L. Ji, A. X. Liu, and J. Wang. Characterizing and modeling internet traffic dynamics of cellular devices. In Proceedings of the ACM SIGMETRICS Joint International Conference on Measurement and Modeling of Computer Systems, pages 305-316. ACM, 2011.

[226] L. S. Shapley. A value for n-person games. Contributions to the theory of games, 2:307-317, Princeton University Press. 1953.

[227] L. S. Shapley. Cores of convex games. International Journal of Game Theory, 1(1):11-26, Springer. 1971.

[228] A. Sharma, V. Navda, R. Ramjee, V. N. Padmanabhan, and E. M. Belding. Cool-Tether: energy efficient on-the-fly WiFi hot-spots using mobile phones. In Proceedings of the 5th International Conference on Emerging Networking Experiments and Technologies, CoNEXT '09. ACM, 2009.

[229] E. Shih, P. Bahl, and M. J. Sinclair. Wake on wireless: an event driven energy saving strategy for battery operated devices. In Proceedings of the 8th Annual International Conference on Mobile Computing and Networking, MobiCom '02, pages 160-171. ACM, 2002.

[230] A. Sieber and J. Nolte. Online device-level energy accounting for wireless sensor nodes. In Proceedings of the 10th European Conference on Wireless Sensor Networks, EWSN'13, pages 149-164. SpringerVerlag, 2013.

[231] M. Siekkinen, M. A. Hoque, J. K. Nurminen, and M. Aalto. Streaming over 3G and LTE: How to save smartphone energy in radio access network-friendly way. In Proceedings of the 5th Workshop on Mobile Video, MoVid '13, pages 13-18. ACM, 2013.

[232] D. Soldani, D. Chiavelli, J. Laiho, M. Li, N. Muhammad, G. Giambiasi, and C. Rodriquez. QoE and QoS Monitoring, pages 315-384. John Wiley \& Sons, Ltd, 2006.

[233] K. Taniuchi, Y. Ohba, V. Fajardo, S. Das, M. Tauil, Y.-H. Cheng, A. Dutta, D. Baker, M. Yajnik, and D. Famolari. IEEE 802.21: media independent handover: features, applicability, and realization. IEEE Communications Magazine, 47:112-120, Jan. 2009. 
[234] Larry Page: Your Android battery life should last a day. If it's not, then blame your apps, says Google co-founder. Techradar, 2010. Accessed 26th May 2015.

http: //www.techradar.com/news/phone-and-communications/

mobile-phones/larry-page-your-android-battery-shouldlast-a-day-690439.

[235] Tektronix. Oscilloscopes, accessed 20th February, 2014. http://www.tek.com/oscilloscope.

[236] S. Thakur, M. Saha, A. Singh, and Y. Agarwal. Wattshare: Detailed energy apportionment in shared living spaces within commercial buildings. In Proceedings of the 1st ACM Conference on Embedded Systems for Energy-Efficient Buildings, BuildSys '14, pages 30-39. ACM, 2014.

[237] Apps behind Android problems, says Motorola. The Telegraph, 2011. Accessed 26th May 2015.

http://www.telegraph.co.uk/technology/news/8555589/Appsbehind-Android-problems-says-Motorola.html.

[238] S. H. Tijs and T. S. H. Driessen. Game theory and cost allocation problems. Manage. Sci., 32(8):1015-1028, Aug. INFORMS. 1986.

[239] J. Torsner and J. Bergstrom. Direct transition to cell dynamic host configuration (DCH), 112012. http://www.lens.org/lens/patent/US_8320929_B2.

[240] S. Trifunovic, B. Distl, D. Schatzmann, and F. Legendre. WiFi-Opp: ad-hoc-less opportunistic networking. In Proceedings of the 6th ACM workshop on Challenged networks, CHANTS '11. ACM, 2011.

[241] S. Trifunovic, M. Kurant, K. A. Hummel, and F. Legendre. Wlanopp: Ad-hoc-less opportunistic networking on smartphones. Ad Hoc Networks, 25, Part B:346 - 358, 2015.

[242] S. Trifunovic, A. Picu, T. Hossmann, and K. A. Hummel. Adaptive role switching for fair and efficient battery usage in device-todevice communication. SIGMOBILE Mob. Comput. Commun. Rev., 18(1):25-36, Feb. ACM. 2014.

[243] Y.-t. Tsao, C.-C. Huang, and J. Y.-j. Hsu. Demand-based Apportionment on Electricity Payment of Hvac Systems. In Proceedings of the 2014 International Conference on Autonomous Agents and Multiagent Systems, AAMAS '14, pages 1601-1602. International Foundation for Autonomous Agents and Multiagent Systems, 2014.

[244] tshark - The Wireshark Network Analyzer 1.12.2, accessed 24th May 2015.

https ://www.wireshark.org/docs/man-pages/tshark.html. 
[245] A. Ukhanova, E. Belyaev, L. Wang, and S. Forchhammer. Power consumption analysis of constant bit rate video transmission over $3 \mathrm{G}$ networks. Computer Communications, 35(14):1695 - 1706, 2012. Special issue: Wireless Green Communications and Networking.

[246] N. Vallina-Rodriguez, A. Auçinas, M. Almeida, Y. Grunenberger, K. Papagiannaki, and J. Crowcroft. RILAnalyzer: A comprehensive 3G monitor on your phone. In Proceedings of the 2013 Conference on Internet Measurement Conference, IMC '13, pages 257-264. ACM, 2013.

[247] N. Vallina-Rodriguez and J. Crowcroft. Erdos: Achieving energy savings in mobile os. In Proceedings of the Sixth International Workshop on MobiArch, MobiArch '11, pages 37-42. ACM, 2011.

[248] N. Vallina-Rodriguez and J. Crowcroft. Energy management techniques in modern mobile handsets. Communications Surveys Tutorials, 15(1):179-198, IEEE, 2013.

[249] N. Vallina-Rodriguez, P. Hui, J. Crowcroft, and A. Rice. Exhausting battery statistics: Understanding the energy demands on mobile handsets. In Proceedings of the Second ACM SIGCOMM Workshop on Networking, Systems, and Applications on Mobile Handhelds, MobiHeld '10, pages 9-14. ACM, 2010.

[250] R. van den Brink. Null or nullifying players: The difference between the shapley value and equal division solutions. Journal of Economic Theory, 136(1):767 - 775, Elsevier. 2007.

[251] E. J. Vergara and S. Nadjm-Tehrani. Watts2share: Energy-aware traffic consolidation. In International Conference on Green Computing and Communications (GreenCom), 2013, pages 14-22, Aug IEEE. 2013.

[252] E. J. Vergara, S. Nadjm-Tehrani, M. Asplund, and U. Zurutuza. Resource footprint of a manycast protocol implementation on multiple mobile platforms. In Proceedings of the 2011 Fifth International Conference on Next Generation Mobile Applications, Services and Technologies, NGMAST '11, pages 154-160. IEEE, 2011.

[253] Voodoo RRC Tool App, 2012. Accessed 26th May 2015. https://github.com/supercurio/voodoo-rrc-tool-app.

[254] D. T. Wagner, A. Rice, and A. R. Beresford. Device analyzer: Understanding smartphone usage. In 10th International Conference Mobile and Ubiquitous Systems: Computing, Networking, and Services: MOBIQUITOUS 2013, pages 195-208. Springer International Publishing, 2013. 
[255] L. Wang and J. Manner. Evaluation of data compression for energyaware communication in mobile networks. In International Conference on Cyber-Enabled Distributed Computing and Knowledge Discovery, CyberC '09, pages 69 -76. IEEE, Oct. 2009.

[256] L. Wang and J. Manner. Energy consumption analysis of WLAN, 2G and $3 \mathrm{G}$ interfaces. In International Conference on Green Computing and Communications (GreenCom), 2010, pages 300-307. IEEE, 2010.

[257] N. Warty, R. K. Sheshadri, W. Zheng, and D. Koutsonikolas. A first look at $802.11 n$ power consumption in smartphones. In Proceedings of the First ACM International Workshop on Practical Issues and Applications in Next Generation Wireless Networks, PINGEN '12, pages 27-32. ACM, 2012.

[258] C. Wilke, S. Götz, and S. Richly. Jouleunit: A generic framework for software energy profiling and testing. In Proceedings of the 2013 Workshop on Green in/by Software Engineering, GIBSE '13, pages 9-14. ACM, 2013.

[259] C. Wilke, S. Richly, S. Gotz, C. Piechnick, and U. Assmann. Energy consumption and efficiency in mobile applications: A user feedback study. In International Conference on Green Computing and Communications (GreenCom), 2013, pages 134-141, Aug. IEEE. 2013.

[260] C. Wilke, S. Richly, G. Püschel, C. Piechnick, S. Götz, and U. Aßmannn. Energy Labels for Mobile Applications. In Proceedings of 1. Workshop zur Entwicklung energiebewusster Software / First Workshop for the Development of Energy-aware Software (EEbS 2012), volume 208 of Lecture Notes in Informatics, pages 93-102. Gesselschaft für Informatik, 2012.

[261] H. Wu, K. Tan, J. Liu, and Y. Zhang. Footprint: cellular assisted WiFi AP discovery on mobile phones for energy saving. In Proceedings of the 4th ACM International Workshop on Experimental Evaluation and Characterization, WINTECH '09, pages 67-76. ACM, 2009.

[262] L. Xiang, J. Luo, and C. Rosenberg. Compressed data aggregation: Energy-efficient and high-fidelity data collection. IEEE/ACM Transactions on Networking, 21(6):1722-1735, 2013.

[263] Y. Xiao, R. Kalyanaraman, and A. Yla-Jaaski. Energy consumption of mobile YouTube: Quantitative measurement and analysis. In Proceedings the 2008 Second International Conference on Next Generation Mobile Applications, Services and Technologies, NGMAST '08, pages 61-69. IEEE, Sept. 2008.

[264] Y. Xiao, P. Savolainen, A. Karppanen, M. Siekkinen, and A. YläJääski. Practical power modeling of data transmission over $802.11 \mathrm{~g}$ 
for wireless applications. In Proceedings of the 1st International Conference on Energy-Efficient Computing and Networking, e-Energy '10, pages 75-84. ACM, 2010.

[265] Z. Xiao, L. Guo, and J. Tracey. Understanding instant messaging traffic characteristics. In Proceedings of the 27th International Conference on Distributed Computing Systems, ICDCS '07, pages 51-59. IEEE, 2007.

[266] E. Yaacoub, H. Ghazzai, M.-S. Alouini, and A. Abu-Dayya. Achieving energy efficiency in LTE with joint D2D communications and green networking techniques. In 9th International Wireless Communications and Mobile Computing Conference (IWCMC), 2013, pages 270-275, IEEE. 2013.

[267] S.-R. Yang, S.-Y. Yan, and H.-N. Hung. Modeling UMTS power saving with bursty packet data traffic. Mobile Computing, IEEE Transactions on, 6(12):1398-1409, Dec. 2007.

[268] J.-H. Yeh, J.-C. Chen, and C.-C. Lee. Comparative analysis of energysaving techniques in $3 \mathrm{GPP}$ and $3 \mathrm{GPP} 2$ systems. IEEE Transactions on Vehicular Technology, 58(1):432-448, 2009.

[269] J. W. Yoo and K. H. Park. A cooperative clustering protocol for energy saving of mobile devices with wlan and bluetooth interfaces. IEEE Transactions on Mobile Computing, 10:491-504, April 2011.

[270] A. Zanella. A mathematical framework for the performance analysis of Bluetooth with enhanced data rate. IEEE Transactions on Communications, 57(8):2463-2473, Aug. 2009.

[271] A. Zanella and F. D. Pellegrini. Mathematical analysis of IEEE 802.11 energy efficiency. In Proceedings of the 7th International Symposium on Wireless Personal Multimedia Communications. IEEE, 2004.

[272] H. Zeng, C. S. Ellis, A. R. Lebeck, and A. Vahdat. ECOSystem: Managing Energy As a First Class Operating System Resource. SIGOPS Oper. Syst. Rev., 36(5):123-132, ACM, Oct. 2002.

[273] H. Zeng, C. S. Ellis, A. R. Lebeck, and A. Vahdat. Currentcy: A unifying abstraction for expressing energy management policies. In Proceedings of the Annual Conference on USENIX Annual Technical Conference, ATEC '03, pages 4-4. USENIX Association, 2003.

[274] H. Zeng, C. S. Ellis, A. R. Lebeck, and A. Vahdat. Ecosystem: Managing energy as a first class operating system resource. SIGARCH Comput. Archit. News, 30(5):123-132, Oct. ACM. 2002. 
[275] L. Zhang, B. Tiwana, Z. Qian, Z. Wang, R. P. Dick, Z. M. Mao, and L. Yang. Accurate online power estimation and automatic battery behavior based power model generation for smartphones. In Proceedings of the Eighth IEEE/ACM/IFIP International Conference on Hardware/software Codesign and System Synthesis, CODES/ISSS '10, pages 105-114. ACM, 2010.

[276] N. Zhang, Y. Lee, M. Radhakrishnan, and R. K. Balan. GameOn: P2P gaming on public transport. In Proceedings of the 13th Annual International Conference on Mobile Systems, Applications, and Services, MobiSys '15, pages 105-119. ACM, 2015.

[277] T. Zhang, Y.-J. Chen, C.-W. Chang, C.-Y. Yang, T.-W. Kuo, and T. Chen. Power management strategies in data transmission. In Proceedings of the 16th Asia and South Pacific Design Automation Conference, ASPDAC '11, pages 668-675. IEEE, 2011.

[278] L. Zhong and N. K. Jha. Graphical user interface energy characterization for handheld computers. In Proceedings of the 2003 International Conference on Compilers, Architecture and Synthesis for Embedded Systems, CASES '03, pages 232-242. ACM, 2003.

[279] J. Zhou, M. Li, L. Liu, X. She, and L. Chen. Energy source aware target cell selection and coverage optimization for power saving in cellular networks. In International Conference on Green Computing and Communications (GreenCom), 2010, pages 1-8. IEEE, 2010.

[280] L. Zhou, H. Xu, H. Tian, Y. Gao, L. Du, and L. Chen. Performance analysis of power saving mechanism with adjustable DRX cycles in 3GPP LTE. In IEEE 68th Vehicular Technology Conference, 2008. VTC 2008-Fall., pages 1-5, Sept 2008.

[281] Z. Zhuang, K.-H. Kim, and J. P. Singh. Improving Energy Efficiency of Location Sensing on Smartphones. In Proceedings of the 8th international conference on Mobile systems, applications, and services, MobiSys '10, pages 315-330. ACM, 2010. 

Department of Computer and Information Science

Linköpings universitet

\section{Dissertations}

\section{Linköping Studies in Science and Technology \\ Linköping Studies in Arts and Science \\ Linköping Studies in Statistics \\ Linköpings Studies in Information Science}

Linköping Studies in Science and Technology

No 14 Anders Haraldsson: A Program Manipulation System Based on Partial Evaluation, 1977, ISBN 917372-144-1.

No 17 Bengt Magnhagen: Probability Based Verification of Time Margins in Digital Designs, 1977, ISBN 91-7372157-3.

No 18 Mats Cedwall: Semantisk analys av processbeskrivningar i naturligt språk, 1977, ISBN 91- 7372168-9.

No 22 Jaak Urmi: A Machine Independent LISP Compiler and its Implications for Ideal Hardware, 1978, ISBN 91-7372-188-3.

No 33 Tore Risch: Compilation of Multiple File Queries in a Meta-Database System 1978, ISBN 91- 7372-232-4.

No 51 Erland Jungert: Synthesizing Database Structures from a User Oriented Data Model, 1980, ISBN 917372-387-8.

No 54 Sture Hägglund: Contributions to the Development of Methods and Tools for Interactive Design of Applications Software, 1980, ISBN 91-7372-404-1.

No 55 Pär Emanuelson: Performance Enhancement in a Well-Structured Pattern Matcher through Partial Evaluation, 1980, ISBN 91-7372-403-3.

No 58 Bengt Johnsson, Bertil Andersson: The HumanComputer Interface in Commercial Systems, 1981, ISBN 91-7372-414-9.

No 69 H. Jan Komorowski: A Specification of an Abstract Prolog Machine and its Application to Partial Evaluation, 1981, ISBN 91-7372-479-3.

No 71 René Reboh: Knowledge Engineering Techniques and Tools for Expert Systems, 1981, ISBN 91-7372489-0.

No 77 Östen Oskarsson: Mechanisms of Modifiability in large Software Systems, 1982, ISBN 91- 7372-527-7.

No 94 Hans Lunell: Code Generator Writing Systems, 1983, ISBN 91-7372-652-4.

No 97 Andrzej Lingas: Advances in Minimum Weight Triangulation, 1983, ISBN 91-7372-660-5.

No 109 Peter Fritzson: Towards a Distributed Programming Environment based on Incremental Compilation, 1984, ISBN 91-7372-801-2.

No 111 Erik Tengvald: The Design of Expert Planning Systems. An Experimental Operations Planning System for Turning, 1984, ISBN 91-7372- 805-5.

No 155 Christos Levcopoulos: Heuristics for Minimum Decompositions of Polygons, 1987, ISBN 91-7870133-3.

No 165 James W. Goodwin: A Theory and System for NonMonotonic Reasoning, 1987, ISBN 91-7870-183-X.

No 170 Zebo Peng: A Formal Methodology for Automated Synthesis of VLSI Systems, 1987, ISBN 91-7870-225-9.

No 174 Johan Fagerström: A Paradigm and System for Design of Distributed Systems, 1988, ISBN 91-7870301-8.
No 192 Dimiter Driankov: Towards a Many Valued Logic of Quantified Belief, 1988, ISBN 91-7870-374-3.

No 213 Lin Padgham: Non-Monotonic Inheritance for an Object Oriented Knowledge Base, 1989, ISBN 917870-485-5.

No 214 Tony Larsson: A Formal Hardware Description and Verification Method, 1989, ISBN 91-7870-517-7.

No 221 Michael Reinfrank: Fundamentals and Logical Foundations of Truth Maintenance, 1989, ISBN 917870-546-0.

No 239 Jonas Löwgren: Knowledge-Based Design Support and Discourse Management in User Interface Management Systems, 1991, ISBN 91-7870-720-X.

No 244 Henrik Eriksson: Meta-Tool Support for Knowledge Acquisition, 1991, ISBN 91-7870-746-3.

No 252 Peter Eklund: An Epistemic Approach to Interactive Design in Multiple Inheritance Hierarchies, 1991, ISBN 91-7870-784-6.

No 258 Patrick Doherty: NML3 - A Non-Monotonic Formalism with Explicit Defaults, 1991, ISBN 917870-816-8.

No 260 Nahid Shahmehri: Generalized Algorithmic Debugging, 1991, ISBN 91-7870-828-1.

No 264 Nils Dahlbäck: Representation of DiscourseCognitive and Computational Aspects, 1992, ISBN 91-7870-850-8.

No 265 Ulf Nilsson: Abstract Interpretations and Abstract Machines: Contributions to a Methodology for the Implementation of Logic Programs, 1992, ISBN 917870-858-3.

No 270 Ralph Rönnquist: Theory and Practice of Tensebound Object References, 1992, ISBN 91-7870-873-7.

No 273 Björn Fjellborg: Pipeline Extraction for VLSI Data Path Synthesis, 1992, ISBN 91-7870-880-X.

No 276 Staffan Bonnier: A Formal Basis for Horn Clause Logic with External Polymorphic Functions, 1992, ISBN 91-7870-896-6.

No 277 Kristian Sandahl: Developing Knowledge Management Systems with an Active Expert Methodology, 1992, ISBN 91-7870-897-4.

No 281 Christer Bäckström: Computational Complexity of Reasoning about Plans, 1992, ISBN 91-7870-979-2.

No 292 Mats Wirén: Studies in Incremental Natural Language Analysis, 1992, ISBN 91-7871-027-8.

No 297 Mariam Kamkar: Interprocedural Dynamic Slicing with Applications to Debugging and Testing, 1993, ISBN 91-7871-065-0.

No 302 Tingting Zhang: A Study in Diagnosis Using Classification and Defaults, 1993, ISBN 91-7871-078-2

No 312 Arne Jönsson: Dialogue Management for Natural Language Interfaces - An Empirical Approach, 1993, ISBN 91-7871-110-X.

No 338 Simin Nadjm-Tehrani: Reactive Systems in Physical Environments: Compositional Modelling and Framework for Verification, 1994, ISBN 91-7871-237-8. 
No 371 Bengt Savén: Business Models for Decision Support and Learning. A Study of Discrete-Event Manufacturing Simulation at Asea/ABB 1968-1993, 1995, ISBN 91-7871-494-X.

No 375 Ulf Söderman: Conceptual Modelling of Mode Switching Physical Systems, 1995, ISBN 91-7871-5164.

No 383 Andreas Kågedal: Exploiting Groundness in Logic Programs, 1995, ISBN 91-7871-538-5.

No 396 George Fodor: Ontological Control, Description, Identification and Recovery from Problematic Control Situations, 1995, ISBN 91-7871-603-9.

No 413 Mikael Pettersson: Compiling Natural Semantics, 1995, ISBN 91-7871-641-1.

No 414 Xinli Gu: RT Level Testability Improvement by Testability Analysis and Transformations, 1996, ISBN 91-7871-654-3.

No 416 Hua Shu: Distributed Default Reasoning, 1996, ISBN 91-7871-665-9.

No 429 Jaime Villegas: Simulation Supported Industrial Training from an Organisational Learning Perspective - Development and Evaluation of the SSIT Method, 1996, ISBN 91-7871-700-0.

No 431 Peter Jonsson: Studies in Action Planning: Algorithms and Complexity, 1996, ISBN 91-7871-7043.

No 437 Johan Boye: Directional Types in Logic Programming, 1996, ISBN 91-7871-725-6.

No 439 Cecilia Sjöberg: Activities, Voices and Arenas: Participatory Design in Practice, 1996, ISBN 91-7871728-0.

No 448 Patrick Lambrix: Part-Whole Reasoning in Description Logics, 1996, ISBN 91-7871-820-1.

No 452 Kjell Orsborn: On Extensible and Object-Relational Database Technology for Finite Element Analysis Applications, 1996, ISBN 91-7871-827-9.

No 459 Olof Johansson: Development Environments for Complex Product Models, 1996, ISBN 91-7871-855-4.

No 461 Lena Strömbäck: User-Defined Constructions in Unification-Based Formalisms, 1997, ISBN 91-7871857-0.

No 462 Lars Degerstedt: Tabulation-based Logic Programming: A Multi-Level View of Query Answering, 1996, ISBN 91-7871-858-9.

No 475 Fredrik Nilsson: Strategi och ekonomisk styrning En studie av hur ekonomiska styrsystem utformas och används efter företagsförvärv, 1997, ISBN 917871-914-3.

No 480 Mikael Lindvall: An Empirical Study of Requirements-Driven Impact Analysis in Object-Oriented Software Evolution, 1997, ISBN 91-7871-927-5.

No 485 Göran Forslund: Opinion-Based Systems: The Cooperative Perspective on Knowledge-Based Decision Support, 1997, ISBN 91-7871-938-0.

No 494 Martin Sköld: Active Database Management Systems for Monitoring and Control, 1997, ISBN 917219-002-7.

No 495 Hans Olsén: Automatic Verification of Petri Nets in a CLP framework, 1997, ISBN 91-7219-011-6.

No 498 Thomas Drakengren: Algorithms and Complexity for Temporal and Spatial Formalisms, 1997, ISBN 917219-019-1.

No 502 Jakob Axelsson: Analysis and Synthesis of Heterogeneous Real-Time Systems, 1997, ISBN 91-7219-035-3.
No 503 Johan Ringström: Compiler Generation for DataParallel Programming Languages from Two-Level Semantics Specifications, 1997, ISBN 91-7219-045-0.

No 512 Anna Moberg: Närhet och distans - Studier av kommunikationsmönster i satellitkontor och flexibla kontor, 1997, ISBN 91-7219-119-8.

No 520 Mikael Ronström: Design and Modelling of a Parallel Data Server for Telecom Applications, 1998, ISBN 91-7219-169-4

No 522 Niclas Ohlsson: Towards Effective Fault Prevention - An Empirical Study in Software Engineering, 1998, ISBN 91-7219-176-7.

No 526 Joachim Karlsson: A Systematic Approach for Prioritizing Software Requirements, 1998, ISBN 917219-184-8.

No 530 Henrik Nilsson: Declarative Debugging for Lazy Functional Languages, 1998, ISBN 91-7219-197-x.

No 555 Jonas Hallberg: Timing Issues in High-Level Synthesis, 1998, ISBN 91-7219-369-7.

No 561 Ling Lin: Management of 1-D Sequence Data - From Discrete to Continuous, 1999, ISBN 91-7219-402-2.

No 563 Eva L Ragnemalm: Student Modelling based on Collaborative Dialogue with a Learning Companion, 1999, ISBN 91-7219-412-X.

No 567 Jörgen Lindström: Does Distance matter? On geographical dispersion in organisations, 1999, ISBN 917219-439-1.

No 582 Vanja Josifovski: Design, Implementation and Evaluation of a Distributed Mediator System for Data Integration, 1999, ISBN 91-7219-482-0.

No 589 Rita Kovordányi: Modeling and Simulating Inhibitory Mechanisms in Mental Image Reinterpretation - Towards Cooperative HumanComputer Creativity, 1999, ISBN 91-7219-506-1.

No 592 Mikael Ericsson: Supporting the Use of Design Knowledge - An Assessment of Commenting Agents, 1999, ISBN 91-7219-532-0.

No 593 Lars Karlsson: Actions, Interactions and Narratives, 1999, ISBN 91-7219-534-7.

No 594 C. G. Mikael Johansson: Social and Organizational Aspects of Requirements Engineering Methods - A practice-oriented approach, 1999, ISBN 91-7219-541$\mathrm{X}$.

No 595 Jörgen Hansson: Value-Driven Multi-Class Overload Management in Real-Time Database Systems, 1999, ISBN 91-7219-542-8.

No 596 Niklas Hallberg: Incorporating User Values in the Design of Information Systems and Services in the Public Sector: A Methods Approach, 1999, ISBN 917219-543-6

No 597 Vivian Vimarlund: An Economic Perspective on the Analysis of Impacts of Information Technology: From Case Studies in Health-Care towards General Models and Theories, 1999, ISBN 91-7219-544-4.

No 598 Johan Jenvald: Methods and Tools in ComputerSupported Taskforce Training, 1999, ISBN 91-7219547-9.

No 607 Magnus Merkel: Understanding and enhancing translation by parallel text processing, 1999, ISBN 917219-614-9.

No 611 Silvia Coradeschi: Anchoring symbols to sensory data, 1999, ISBN 91-7219-623-8.

No 613 Man Lin: Analysis and Synthesis of Reactive Systems: A Generic Layered Architecture Perspective, 1999, ISBN 91-7219-630-0. 
No 618 Jimmy Tjäder: Systemimplementering i praktiken En studie av logiker i fyra projekt, 1999, ISBN 917219-657-2.

No 627 Vadim Engelson: Tools for Design, Interactive Simulation, and Visualization of Object-Oriented Models in Scientific Computing, 2000, ISBN 91-7219709-9.

No 637 Esa Falkenroth: Database Technology for Control and Simulation, 2000, ISBN 91-7219-766-8.

No 639 Per-Arne Persson: Bringing Power and Knowledge Together: Information Systems Design for Autonomy and Control in Command Work, 2000, ISBN 91-7219796-X.

No 660 Erik Larsson: An Integrated System-Level Design for Testability Methodology, 2000, ISBN 91-7219-890-7.

No 688 Marcus Bjäreland: Model-based Execution Monitoring, 2001, ISBN 91-7373-016-5.

No 689 Joakim Gustafsson: Extending Temporal Action Logic, 2001, ISBN 91-7373-017-3.

No 720 Carl-Johan Petri: Organizational Information Provision - Managing Mandatory and Discretionary Use of Information Technology, 2001, ISBN-91-7373-1269.

No 724 Paul Scerri: Designing Agents for Systems with Adjustable Autonomy, 2001, ISBN 9173732079.

No 725 Tim Heyer: Semantic Inspection of Software Artifacts: From Theory to Practice, 2001, ISBN 91 73732087.

No 726 Pär Carlshamre: A Usability Perspective on Requirements Engineering - From Methodology to Product Development, 2001, ISBN 9173732125.

No 732 Juha Takkinen: From Information Management to Task Management in Electronic Mail, 2002, ISBN 91 73732583.

No 745 Johan Åberg: Live Help Systems: An Approach to Intelligent Help for Web Information Systems, 2002, ISBN 91-7373-311-3.

No 746 Rego Granlund: Monitoring Distributed Teamwork Training, 2002, ISBN 91-7373-312-1.

No 757 Henrik André-Jönsson: Indexing Strategies for Time Series Data, 2002, ISBN 917373-346-6.

No 747 Anneli Hagdahl: Development of IT-supported Interorganisational Collaboration - A Case Study in the Swedish Public Sector, 2002, ISBN 91-7373-314-8.

No 749 Sofie Pilemalm: Information Technology for NonProfit Organisations - Extended Participatory Design of an Information System for Trade Union Shop Stewards, 2002, ISBN 91-7373-318-0.

No 765 Stefan Holmlid: Adapting users: Towards a theory of use quality, 2002, ISBN 91-7373-397-0.

No 771 Magnus Morin: Multimedia Representations of Distributed Tactical Operations, 2002, ISBN 91-7373-4217.

No 772 Pawel Pietrzak: A Type-Based Framework for Locating Errors in Constraint Logic Programs, 2002, ISBN 91-7373-422-5.

No 758 Erik Berglund: Library Communication Among Programmers Worldwide, 2002, ISBN 91-7373-349-0.

No 774 Choong-ho Yi: Modelling Object-Oriented Dynamic Systems Using a Logic-Based Framework, 2002, ISBN 91-7373-424-1.

No 779 Mathias Broxvall: A Study in the Computational Complexity of Temporal Reasoning, 2002, ISBN 917373-440-3.
No 793 Asmus Pandikow: A Generic Principle for Enabling Interoperability of Structured and Object-Oriented Analysis and Design Tools, 2002, ISBN 91-7373-479-9.

No 785 Lars Hult: Publika Informationstjänster. En studie av den Internetbaserade encyklopedins bruksegenskaper, 2003, ISBN 91-7373-461-6.

No 800 Lars Taxén: A Framework for the Coordination of Complex Systems' Development, 2003, ISBN 917373-604-X

No 808 Klas Gäre: Tre perspektiv på förväntningar och förändringar i samband med införande av informationssystem, 2003, ISBN 91-7373-618-X.

No 821 Mikael Kindborg: Concurrent Comics programming of social agents by children, 2003, ISBN 91-7373-651-1.

No 823 Christina Ölvingson: On Development of Information Systems with GIS Functionality in Public Health Informatics: A Requirements Engineering Approach, 2003, ISBN 91-7373-656-2.

No 828 Tobias Ritzau: Memory Efficient Hard Real-Time Garbage Collection, 2003, ISBN 91-7373-666-X.

No 833 Paul Pop: Analysis and Synthesis of Communication-Intensive Heterogeneous Real-Time Systems, 2003, ISBN 91-7373-683-X.

No 852 Johan Moe: Observing the Dynamic Behaviour of Large Distributed Systems to Improve Development and Testing - An Empirical Study in Software Engineering, 2003, ISBN 91-7373-779-8.

No 867 Erik Herzog: An Approach to Systems Engineering Tool Data Representation and Exchange, 2004, ISBN 91-7373-929-4.

No 872 Aseel Berglund: Augmenting the Remote Control: Studies in Complex Information Navigation for Digital TV, 2004, ISBN 91-7373-940-5.

No 869 Jo Skåmedal: Telecommuting's Implications on Travel and Travel Patterns, 2004, ISBN 91-7373-935-9.

No 870 Linda Askenäs: The Roles of IT - Studies of Organising when Implementing and Using Enterprise Systems, 2004, ISBN 91-7373-936-7.

No 874 Annika Flycht-Eriksson: Design and Use of Ontologies in Information-Providing Dialogue Systems, 2004, ISBN 91-7373-947-2.

No 873 Peter Bunus: Debugging Techniques for EquationBased Languages, 2004, ISBN 91-7373-941-3.

No 876 Jonas Mellin: Resource-Predictable and Efficient Monitoring of Events, 2004, ISBN 91-7373-956-1.

No 883 Magnus Bång: Computing at the Speed of Paper: Ubiquitous Computing Environments for Healthcare Professionals, 2004, ISBN 91-7373-971-5

No 882 Robert Eklund: Disfluency in Swedish humanhuman and human-machine travel booking dialogues, 2004, ISBN 91-7373-966-9.

No 887 Anders Lindström: English and other Foreign Linguistic Elements in Spoken Swedish. Studies of Productive Processes and their Modelling using Finite-State Tools, 2004, ISBN 91-7373-981-2.

No 889 Zhiping Wang: Capacity-Constrained Production-inventory systems - Modelling and Analysis in both a traditional and an e-business context, 2004, ISBN 9185295-08-6

No 893 Pernilla Qvarfordt: Eyes on Multimodal Interaction, 2004, ISBN 91-85295-30-2.

No 910 Magnus Kald: In the Borderland between Strategy and Management Control - Theoretical Framework and Empirical Evidence, 2004, ISBN 91-85295-82-5. 
No 918 Jonas Lundberg: Shaping Electronic News: Genre Perspectives on Interaction Design, 2004, ISBN 9185297-14-3.

No 900 Mattias Arvola: Shades of use: The dynamics of interaction design for sociable use, 2004, ISBN 9185295-42-6.

No 920 Luis Alejandro Cortés: Verification and Scheduling Techniques for Real-Time Embedded Systems, 2004, ISBN 91-85297-21-6.

No 929 Diana Szentivanyi: Performance Studies of FaultTolerant Middleware, 2005, ISBN 91-85297-58-5.

No 933 Mikael Cäker: Management Accounting as Constructing and Opposing Customer Focus: Three Case Studies on Management Accounting and Customer Relations, 2005, ISBN 91-85297-64-X.

No 937 Jonas Kvarnström: TALplanner and Other Extensions to Temporal Action Logic, 2005, ISBN 9185297-75-5.

No 938 Bourhane Kadmiry: Fuzzy Gain-Scheduled Visual Servoing for Unmanned Helicopter, 2005, ISBN 9185297-76-3.

No 945 Gert Jervan: Hybrid Built-In Self-Test and Test Generation Techniques for Digital Systems, 2005, ISBN: 91-85297-97-6.

No 946 Anders Arpteg: Intelligent Semi-Structured Information Extraction, 2005, ISBN 91-85297-98-4.

No 947 Ola Angelsmark: Constructing Algorithms for Constraint Satisfaction and Related Problems - Methods and Applications, 2005, ISBN 91-85297-99-2.

No 963 Calin Curescu: Utility-based Optimisation of Resource Allocation for Wireless Networks, 2005, ISBN 91-85457-07-8.

No 972 Björn Johansson: Joint Control in Dynamic Situations, 2005, ISBN 91-85457-31-0.

No 974 Dan Lawesson: An Approach to Diagnosability Analysis for Interacting Finite State Systems, 2005, ISBN 91-85457-39-6.

No 979 Claudiu Duma: Security and Trust Mechanisms for Groups in Distributed Services, 2005, ISBN 91-85457$54-X$.

No 983 Sorin Manolache: Analysis and Optimisation of Real-Time Systems with Stochastic Behaviour, 2005, ISBN 91-85457-60-4.

No 986 Yuxiao Zhao: Standards-Based Application Integration for Business-to-Business Communications, 2005, ISBN 91-85457-66-3.

No 1004 Patrik Haslum: Admissible Heuristics for Automated Planning, 2006, ISBN 91-85497-28-2.

No 1005 Aleksandra Tešanovic: Developing Reusable and Reconfigurable Real-Time Software using Aspects and Components, 2006, ISBN 91-85497-29-0.

No 1008 David Dinka: Role, Identity and Work: Extending the design and development agenda, 2006, ISBN 9185497-42-8.

No 1009 Iakov Nakhimovski: Contributions to the Modeling and Simulation of Mechanical Systems with Detailed Contact Analysis, 2006, ISBN 91-85497-43-X.

No 1013 Wilhelm Dahllöf: Exact Algorithms for Exact Satisfiability Problems, 2006, ISBN 91-85523-97-6.

No 1016 Levon Saldamli: PDEModelica - A High-Level Language for Modeling with Partial Differential Equations, 2006, ISBN 91-85523-84-4.

No 1017 Daniel Karlsson: Verification of Component-based Embedded System Designs, 2006, ISBN 91-85523-79-8
No 1018 Ioan Chisalita: Communication and Networking Techniques for Traffic Safety Systems, 2006, ISBN 9185523-77-1.

No 1019 Tarja Susi: The Puzzle of Social Activity - The Significance of Tools in Cognition and Cooperation, 2006, ISBN 91-85523-71-2.

No 1021 Andrzej Bednarski: Integrated Optimal Code Generation for Digital Signal Processors, 2006, ISBN 9185523-69-0.

No 1022 Peter Aronsson: Automatic Parallelization of Equation-Based Simulation Programs, 2006, ISBN 9185523-68-2.

No 1030 Robert Nilsson: A Mutation-based Framework for Automated Testing of Timeliness, 2006, ISBN 9185523-35-6.

No 1034 Jon Edvardsson: Techniques for Automatic Generation of Tests from Programs and Specifications, 2006, ISBN 91-85523-31-3.

No 1035 Vaida Jakoniene: Integration of Biological Data, 2006, ISBN 91-85523-28-3.

No 1045 Genevieve Gorrell: Generalized Hebbian Algorithms for Dimensionality Reduction in Natural Language Processing, 2006, ISBN 91-85643-88-2.

No 1051 Yu-Hsing Huang: Having a New Pair of Glasses Applying Systemic Accident Models on Road Safety, 2006, ISBN 91-85643-64-5.

No 1054 Åsa Hedenskog: Perceive those things which cannot be seen - A Cognitive Systems Engineering perspective on requirements management, 2006, ISBN 91-85643-57-2.

No 1061 Cécile Åberg: An Evaluation Platform for Semantic Web Technology, 2007, ISBN 91-85643-31-9.

No 1073 Mats Grindal: Handling Combinatorial Explosion in Software Testing, 2007, ISBN 978-91-85715-74-9.

No 1075 Almut Herzog: Usable Security Policies for Runtime Environments, 2007, ISBN 978-91-85715-65-7.

No 1079 Magnus Wahlström: Algorithms, measures, and upper bounds for Satisfiability and related problems, 2007, ISBN 978-91-85715-55-8.

No 1083 Jesper Andersson: Dynamic Software Architectures, 2007, ISBN 978-91-85715-46-6.

No 1086 Ulf Johansson: Obtaining Accurate and Comprehensible Data Mining Models - An Evolutionary Approach, 2007, ISBN 978-91-85715-34-3.

No 1089 Traian Pop: Analysis and Optimisation of Distributed Embedded Systems with Heterogeneous Scheduling Policies, 2007, ISBN 978-91-85715-27-5.

No 1091 Gustav Nordh: Complexity Dichotomies for CSPrelated Problems, 2007, ISBN 978-91-85715-20-6.

No 1106 Per Ola Kristensson: Discrete and Continuous Shape Writing for Text Entry and Control, 2007, ISBN 97891-85831-77-7.

No 1110 He Tan: Aligning Biomedical Ontologies, 2007, ISBN 978-91-85831-56-2.

No 1112 Jessica Lindblom: Minding the body - Interacting socially through embodied action, 2007, ISBN 978-9185831-48-7.

No 1113 Pontus Wärnestål: Dialogue Behavior Management in Conversational Recommender Systems, 2007, ISBN 978-91-85831-47-0.

No 1120 Thomas Gustafsson: Management of Real-Time Data Consistency and Transient Overloads in Embedded Systems, 2007, ISBN 978-91-85831-33-3. 
No 1127 Alexandru Andrei: Energy Efficient and Predictable Design of Real-time Embedded Systems, 2007, ISBN 978-91-85831-06-7.

No 1139 Per Wikberg: Eliciting Knowledge from Experts in Modeling of Complex Systems: Managing Variation and Interactions, 2007, ISBN 978-91-85895-66-3.

No 1143 Mehdi Amirijoo: QoS Control of Real-Time Data Services under Uncertain Workload, 2007, ISBN 97891-85895-49-6.

No 1150 Sanny Syberfeldt: Optimistic Replication with Forward Conflict Resolution in Distributed Real-Time Databases, 2007, ISBN 978-91-85895-27-4.

No 1155 Beatrice Alenljung: Envisioning a Future Decision Support System for Requirements Engineering - A Holistic and Human-centred Perspective, 2008, ISBN 978-91-85895-11-3.

No 1156 Artur Wilk: Types for XML with Application to Xcerpt, 2008, ISBN 978-91-85895-08-3.

No 1183 Adrian Pop: Integrated Model-Driven Development Environments for Equation-Based Object-Oriented Languages, 2008, ISBN 978-91-7393-895-2.

No 1185 Jörgen Skågeby: Gifting Technologies Ethnographic Studies of End-users and Social Media Sharing, 2008, ISBN 978-91-7393-892-1.

No 1187 Imad-Eldin Ali Abugessaisa: Analytical tools and information-sharing methods supporting road safety organizations, 2008, ISBN 978-91-7393-887-7.

No 1204 H. Joe Steinhauer: A Representation Scheme for Description and Reconstruction of Object Configurations Based on Qualitative Relations, 2008, ISBN 978-91-7393-823-5.

No 1222 Anders Larsson: Test Optimization for Core-based System-on-Chip, 2008, ISBN 978-91-7393-768-9.

No 1238 Andreas Borg: Processes and Models for Capacity Requirements in Telecommunication Systems, 2009, ISBN 978-91-7393-700-9.

No 1240 Fredrik Heintz: DyKnow: A Stream-Based Knowledge Processing Middleware Framework, 2009, ISBN 978-91-7393-696-5.

No 1241 Birgitta Lindström: Testability of Dynamic RealTime Systems, 2009, ISBN 978-91-7393-695-8.

No 1244 Eva Blomqvist: Semi-automatic Ontology Construction based on Patterns, 2009, ISBN 978-91-7393-683-5.

No 1249 Rogier Woltjer: Functional Modeling of Constraint Management in Aviation Safety and Command and Control, 2009, ISBN 978-91-7393-659-0.

No 1260 Gianpaolo Conte: Vision-Based Localization and Guidance for Unmanned Aerial Vehicles, 2009, ISBN 978-91-7393-603-3.

No 1262 AnnMarie Ericsson: Enabling Tool Support for Formal Analysis of ECA Rules, 2009, ISBN 978-91-7393598-2.

No 1266 Jiri Trnka: Exploring Tactical Command and Control: A Role-Playing Simulation Approach, 2009, ISBN 978-91-7393-571-5.

No 1268 Bahlol Rahimi: Supporting Collaborative Work through ICT - How End-users Think of and Adopt Integrated Health Information Systems, 2009, ISBN 978-91-7393-550-0.

No 1274 Fredrik Kuivinen: Algorithms and Hardness Results for Some Valued CSPs, 2009, ISBN 978-91-7393-525-8.

No 1281 Gunnar Mathiason: Virtual Full Replication for Scalable Distributed Real-Time Databases, 2009, ISBN 978-91-7393-503-6.
No 1290 Viacheslav Izosimov: Scheduling and Optimization of Fault-Tolerant Distributed Embedded Systems, 2009, ISBN 978-91-7393-482-4.

No 1294 Johan Thapper: Aspects of a Constraint Optimisation Problem, 2010, ISBN 978-91-7393-464-0.

No 1306 Susanna Nilsson: Augmentation in the Wild: User Centered Development and Evaluation of Augmented Reality Applications, 2010, ISBN 978-917393-416-9.

No 1313 Christer Thörn: On the Quality of Feature Models, 2010, ISBN 978-91-7393-394-0.

No 1321 Zhiyuan He: Temperature Aware and DefectProbability Driven Test Scheduling for System-onChip, 2010, ISBN 978-91-7393-378-0.

No 1333 David Broman: Meta-Languages and Semantics for Equation-Based Modeling and Simulation, 2010, ISBN 978-91-7393-335-3.

No 1337 Alexander Siemers: Contributions to Modelling and Visualisation of Multibody Systems Simulations with Detailed Contact Analysis, 2010, ISBN 978-91-7393317-9.

No 1354 Mikael Asplund: Disconnected Discoveries: Availability Studies in Partitioned Networks, 2010, ISBN 978-91-7393-278-3.

No 1359 Jana Rambusch: Mind Games Extended: Understanding Gameplay as Situated Activity, 2010, ISBN 978-91-7393-252-3.

No 1373 Sonia Sangari: Head Movement Correlates to Focus Assignment in Swedish,2011,ISBN 978-91-7393-154-0.

No 1374 Jan-Erik Källhammer: Using False Alarms when Developing Automotive Active Safety Systems, 2011, ISBN 978-91-7393-153-3.

No 1375 Mattias Eriksson: Integrated Code Generation, 2011, ISBN 978-91-7393-147-2.

No 1381 Ola Leifler: Affordances and Constraints of Intelligent Decision Support for Military Command and Control - Three Case Studies of Support Systems, 2011, ISBN 978-91-7393-133-5.

No 1386 Soheil Samii: Quality-Driven Synthesis and Optimization of Embedded Control Systems, 2011, ISBN 978-91-7393-102-1.

No 1419 Erik Kuiper: Geographic Routing in Intermittentlyconnected Mobile Ad Hoc Networks: Algorithms and Performance Models, 2012, ISBN 978-91-7519981-8.

No 1451 Sara Stymne: Text Harmonization Strategies for Phrase-Based Statistical Machine Translation, 2012, ISBN 978-91-7519-887-3.

No 1455 Alberto Montebelli: Modeling the Role of Energy Management in Embodied Cognition, 2012, ISBN 978-91-7519-882-8.

No 1465 Mohammad Saifullah: Biologically-Based Interactive Neural Network Models for Visual Attention and Object Recognition, 2012, ISBN 978-91-7519-838-5.

No 1490 Tomas Bengtsson: Testing and Logic Optimization Techniques for Systems on Chip, 2012, ISBN 978-917519-742-5.

No 1481 David Byers: Improving Software Security by Preventing Known Vulnerabilities, 2012, ISBN 97891-7519-784-5.

No 1496 Tommy Färnqvist: Exploiting Structure in CSPrelated Problems, 2013, ISBN 978-91-7519-711-1.

No 1503 John Wilander: Contributions to Specification, Implementation, and Execution of Secure Software, 2013, ISBN 978-91-7519-681-7. 
No 1506 Magnus Ingmarsson: Creating and Enabling the Useful Service Discovery Experience, 2013, ISBN 97891-7519-662-6.

No 1547 Wladimir Schamai: Model-Based Verification of Dynamic System Behavior against Requirements: Method, Language, and Tool, 2013, ISBN 978-917519-505-6.

No 1551 Henrik Svensson: Simulations, 2013, ISBN 978-917519-491-2.

No 1559 Sergiu Rafiliu: Stability of Adaptive Distributed Real-Time Systems with Dynamic Resource Management, 2013, ISBN 978-91-7519-471-4.

No 1581 Usman Dastgeer: Performance-aware Component Composition for GPU-based Systems, 2014, ISBN 978-91-7519-383-0.

No 1602 Cai Li: Reinforcement Learning of Locomotion based on Central Pattern Generators, 2014, ISBN 978-917519-313-7.

No 1652 Roland Samlaus: An Integrated Development Environment with Enhanced Domain-Specific Interactive Model Validation, 2015, ISBN 978-917519-090-7.

No 1663 Hannes Uppman: On Some Combinatorial Optimization Problems: Algorithms and Complexity, 2015, ISBN 978-91-7519-072-3.

No 1664 Martin Sjölund: Tools and Methods for Analysis, Debugging, and Performance Improvement of Equation-Based Models, 2015, ISBN 978-91-7519-071-6.

No 1666 Kristian Stavåker: Contributions to Simulation of Modelica Models on Data-Parallel Multi-Core Architectures, 2015, ISBN 978-91-7519-068-6.

No 1680 Adrian Lifa: Hardware/Software Codesign of Embedded Systems with Reconfigurable and Heterogeneous Platforms, 2015, ISBN 978-91-7519-0402.

No 1685 Bogdan Tanasa: Timing Analysis of Distributed Embedded Systems with Stochastic Workload and Reliability Constraints, 2015, ISBN 978-91-7519-022-8.

No 1691 Håkan Warnquist: Troubleshooting Trucks Automated Planning and Diagnosis, 2015, ISBN 97891-7685-993-3.

No 1702 Nima Aghaee: Thermal Issues in Testing of Advanced Systems on Chip, 2015, ISBN 978-91-7685949-0.

No 1715 Maria Vasilevskaya: Security in Embedded Systems: A Model-Based Approach with Risk Metrics, 2015, ISBN 978-91-7685-917-9.

No 1729 Ke Jiang: Security-Driven Design of Real-Time Embedded System, 2016, ISBN 978-91-7685-884-4.

No 1733 Victor Lagerkvist: Strong Partial Clones and the Complexity of Constraint Satisfaction Problems: Limitations and Applications, 2016, ISBN 978-91-7685856-1.

No 1734 Chandan Roy: An Informed System Development Approach to Tropical Cyclone Track and Intensity Forecasting, 2016, ISBN 978-91-7685-854-7.

No 1746 Amir Aminifar: Analysis, Design, and Optimization of Embedded Control Systems, 2016, ISBN 978-917685-826-4.

No 1747 Ekhiotz Vergara: Energy Modelling and Fairness for Efficient Mobile Communication, 2016, ISBN 978-91-7685-822-6.
Linköping Studies in Arts and Science

No 504 Ing-Marie Jonsson: Social and Emotional Characteristics of Speech-based In-Vehicle Information Systems: Impact on Attitude and Driving Behaviour, 2009, ISBN 978-91-7393-478-7.

No 586 Fabian Segelström: Stakeholder Engagement for Service Design: How service designers identify and communicate insights, 2013, ISBN 978-91-7519-554-4.

No 618 Johan Blomkvist: Representing Future Situations of Service: Prototyping in Service Design, 2014, ISBN 978-91-7519-343-4.

No 620 Marcus Mast: Human-Robot Interaction for SemiAutonomous Assistive Robots, 2014, ISBN 978-917519-319-9.

\section{Linköping Studies in Statistics}

No 9 Davood Shahsavani: Computer Experiments Designed to Explore and Approximate Complex Deterministic Models, 2008, ISBN 978-91-7393-976-8.

No 10 Karl Wahlin: Roadmap for Trend Detection and Assessment of Data Quality, 2008, ISBN 978-91-7393$792-4$.

No 11 Oleg Sysoev: Monotonic regression for large multivariate datasets, 2010, ISBN 978-91-7393-412-1.

No 13 Agné Burauskaite-Harju: Characterizing Temporal Change and Inter-Site Correlations in Daily and Subdaily Precipitation Extremes, 2011, ISBN 978-91-7393$110-6$.

\section{Linköping Studies in Information Science}

No 1 Karin Axelsson: Metodisk systemstrukturering- att skapa samstämmighet mellan informationssystemarkitektur och verksamhet, 1998. ISBN-9172-19-296-8.

No 2 Stefan Cronholm: Metodverktyg och användbarhet en studie av datorstödd metodbaserad systemutveckling, 1998, ISBN-9172-19-299-2.

No 3 Anders Avdic: Användare och utvecklare - om anveckling med kalkylprogram, 1999. ISBN-91-7219606-8.

No 4 Owen Eriksson: Kommunikationskvalitet hos informationssystem och affärsprocesser, 2000, ISBN 917219-811-7.

No 5 Mikael Lind: Från system till process - kriterier för processbestämning vid verksamhetsanalys, 2001, ISBN 91-7373-067-X.

No 6 Ulf Melin: Koordination och informationssystem i företag och nätverk, 2002, ISBN 91-7373-278-8.

No 7 Pär J. Ågerfalk: Information Systems Actability - Understanding Information Technology as a Tool for Business Action and Communication, 2003, ISBN 917373-628-7.

No 8 Ulf Seigerroth: Att förstå och förändra systemutvecklingsverksamheter - en taxonomi för metautveckling, 2003, ISBN91-7373-736-4.

No 9 Karin Hedström: Spår av datoriseringens värden Effekter av IT i äldreomsorg, 2004, ISBN 91-7373-9634.

No 10 Ewa Braf: Knowledge Demanded for Action Studies on Knowledge Mediation in Organisations, 2004, ISBN 91-85295-47-7. 
No 11 Fredrik Karlsson: Method Configuration method and computerized tool support, 2005, ISBN 91-8529748-8.

No 12 Malin Nordström: Styrbar systemförvaltning - Att organisera systemförvaltningsverksamhet med hjälp av effektiva förvaltningsobjekt, 2005, ISBN 91-8529760-7.

No 13 Stefan Holgersson: Yrke: POLIS - Yrkeskunskap, motivation, IT-system och andra förutsättningar för polisarbete, 2005, ISBN 91-85299-43-X.

No 14 Benneth Christiansson, Marie-Therese

Christiansson: Mötet mellan process och komponent - mot ett ramverk för en verksamhetsnära kravspecifikation vid anskaffning av komponentbaserade informationssystem, 2006, ISBN 91-8564322-X. 